\title{
DENSITY DEPENDENCE OF ROTATIONAL AND TRANSLATIONAL MOLECULAR DYNAMICS IN LIQUIDS STUDIED BY HIGH PRESSURE NMR
}

\author{
E. W. LANG and H.-D. LÜDEMANN \\ Institut für Biophysik und Physikalische Biochemie, Universität Regensburg, Postfach 10 1042, 93040 \\ Regensburg, Germany
}

(Received 14 November 1992)

\section{CONTENTS}

1. Introduction

2. Experimental Techniques $\quad \mathbf{5 0 8}$

3. Theory 512

3.1. Self-diffusion coefficients $\quad 512$

3.1.1. Self-diffusion and the spin-echo experiment 512

$\begin{array}{ll}\text { 3.1.2. Self-diffusion coefficients in hard sphere fluids } & 513\end{array}$

3.1.3. Self-diffusion coefficients in simple, non-associated liquids $\quad 514$

$\begin{array}{ll}\text { 3.1.4. Self-diffusion coefficients in H-bonded liquids } & 515\end{array}$

3.2. Spin-lattice $\left(T_{1}\right)$ and spin-spin $\left(T_{2}\right)$ relaxation $\quad 517$

$\begin{array}{ll}\text { 3.2.1. Relaxation theory } & 517\end{array}$

$\begin{array}{ll}\text { 3.2.2. Dipole-dipole (DD) relaxation } & 519\end{array}$

3.2.3. Quadrupolar relaxation $\quad 520$

3.2.4. Relaxation through chemical shielding anisotropy (CSA) 522

3.2.5. The spin-rotation relaxation $\quad 522$

3.2.6. Scalar relaxation $\quad 523$

3.3. Chemical exchange 524

4. Chemical Exchange: Experiments 526

4.1. Pressure dependence of the rate constants of chemical reactions 526

4.1.1. Ligand exchange of solvated ions $\quad 526$

4.1.2. Intramolecular conformational transitions $\quad \mathbf{5 2 8}$

5. Self-diffusion Coefficients: Experiments

5.1. Xenon 534

5.2. Lower alkanes $\quad \mathbf{5 3 4}$

5.3. Halomethanes $\quad 537$

5.4. Larger alkanes $\quad 538$

5.5. Miscellaneous non-associating liquids $\quad 542$

5.6. Hydrogen bonded liquids

5.7. Binary mixtures $\quad 549$

6. Relaxation Rates: Experiments $\quad 550$

6.1. Atomic liquids $\quad 550$

6.2. Non-associated liquids $\quad 553$

6.2.1. Simple non-viscous inorganic liquids 553

6.2.2. Miscellaneous non-associating organic liquids $\quad 574$

6.2.3. Complex viscous liquids $\quad 584$

6.3. Hydrogen bonded liquids 588

6.3.1. Alcohols

6.3.2. Ammonia $\quad 590$

6.3.3. Water $\quad 592$

6.4. Aqueous solutions of simple hydrophobic model compounds 606

$\begin{array}{ll}\text { 6.4.1. Water-dioxane mixtures } & 607\end{array}$

$\begin{array}{ll}\text { 6.4.2. Water-dimethylsulphoxide (DMSO) mixtures } & 608\end{array}$

$\begin{array}{ll}\text { 6.4.3. Alcohol-water mixtures } & 609\end{array}$

6.5. Aqueous solutions of simple ionic solutes 611

$\begin{array}{ll}\text { 6.5.1. Self-diffusion in aqueous solutions of atomic ions } & 612\end{array}$

$\begin{array}{ll}\text { 6.5.2. Spin-relaxation in aqueous solutions of atomic ions } & \mathbf{6 1 2}\end{array}$

6.5.3. Reorientational motions of water molecules in undercooled ionic solutions 613 
6.5.4. Multinuclear spin-relaxation in undercooled LiCl- and KF-solutions

$\begin{array}{ll}\text { 6.6. Aqueous solutions of hydrophobic ions } & 620\end{array}$

$\begin{array}{ll}\text { 6.6.1. Dynamics of the water molecules } & 621\end{array}$

$\begin{array}{ll}\text { 6.6.2. Internal flexibility of the } \mathrm{TAA}^{+} \text {-cations } & 622\end{array}$

$\begin{array}{lr}\text { 7. Concluding Remarks } & 623\end{array}$

$\begin{array}{lr}\text { Acknowledgements } & 624\end{array}$

$\begin{array}{lr}\text { References } & 624\end{array}$

\section{INTRODUCTION}

Measurements under high hydrostatic pressure provide the only possibility to separate experimentally the influence of thermal energy from density changes. Due to the low compressibility of normal solids and liquids close to their melting temperature fairly high pressures have to be applied in order to achieve moderate increases in density. Typically pressures in the range of 200 to $400 \mathrm{MPa}$ will increase the density by approximately $10 \%$. In order to maintain a liquid over a wider temperature range under isochoric conditions and/or to study isochoric behaviour at different densities one has thus to be able to apply pressures of at least several $100 \mathrm{MPa}$.

It was recognized in early NMR studies ${ }^{(1)}$ that it would be rewarding to tackle the great experimental difficulties connected with the combination of a high homogeneous magnetic field, a compact metallic autoclave, reliable high pressure RF feedthroughs and sample separating systems. Progress during the following decades rested with a few enthusiasts who patiently developed the technical means. ${ }^{(2-4)}$

At ambient pressure simple liquids, such as the noble gases, methane, and the halomethanes, have a very small liquid range (typically at most $20 \mathrm{~K}$ ), but at high pressure they can be kept at fluid densities over a very wide temperature range, thus permitting the study of a wealth of dynamic properties. The results from NMR studies at high pressure may be compared with results of molecular dynamics simulations in order to test the quality of the potentials used in the computer simulations. Another area of study involves high pressure investigations of chemical kinetics and equilibria which provides information about the volume effects connected with chemical reactions. It has been argued that reaction and activation volumes are easier to obtain precisely and are easier to interpret than entropy data. ${ }^{(5,6)}$ A very special field of research is the study of supercooled water and aqueous solutions, where application of pressure suppresses the homogeneous nucleation temperature by $\sim 50 \mathrm{~K}^{(7,8)}$ thus permitting studies of the relaxation rates of the hydrogen isotopes in the water molecules outside the extreme narrowing limit.

In this review an attempt is made to summarize and evaluate the available 'dynamic' NMR data collected at elevated pressures in various diamagnetic liquids and fluids.

\section{EXPERIMENTAL TECHNIQUES}

The progress made in the field of high pressure NMR has been entirely due to research carried out on 'home-made' equipment. The first successful attempts to characterize the $p, T$-dependence of the molecular mobility were reported in the early 60 s by Benedek. ${ }^{(1)}$ The quantitative evaluation of the results was limited, since the design used in these studies did not permit the complete removal of paramagnetic impurities from the samples, i.e. oxygen and transition metal ions.

In the following only the technical developments specific to NMR applications will be described. For the general design of high pressure apparatus the reader is referred to a recent monograph. ${ }^{(9)}$ Important improvements have occurred in three areas.

(1) Autoclaves for high resolution NMR studies.

(2) Low loss electrical feedthroughs for the RF, which become of increasing importance at the higher fields available in superconducting magnets.

(3) Design of apparatus permitting filling of oxygen free gaseous material into the autoclave and reliable systems to separate the liquids under study from the pressurizing fluid.

High pressure cells, machined from 'non-magnetic' high strength alloys for high resolution studies, permitting an experienced operator to achieve a typical line width of $1 \mathrm{~Hz}$ became available in the $70 \mathrm{~s}$. 
This development was supported by the introduction of superconducting magnets, with improved shim coil design, which allowed a better homogenization of the magnetic field.

Jonas $^{(10,11)}$ and Merbach and his group ${ }^{(3,12,13)}$ were among the first to achieve this aim. At about the same time Yamada ${ }^{(14)}$ introduced a strengthened glass cell design using borosilicate glass capillaries that withstood pressures around $200 \mathrm{MPa}$. This design was later modified to permit spinning of the pressurized sample tube ${ }^{(15,16)}$. The modification of the early glass cell design used by our group has been described by Gaarz et al..$^{(17)}$ and Lang et al. ${ }^{(18)}$

The autoclaves are either machined from high strength titanium alloys or from copper beryllium alloys. The latter have the advantage of a high thermal conductivity, thus reducing the time needed to attain thermal equilibrium. However, after heat treatment they become relatively brittle and susceptible to sudden fractures especially when heated regularly to temperatures above $350 \mathrm{~K}$.

The design developed by Jonas and his group ${ }^{(11)}$ is given in Fig. 1. It has been operated routinely at a field of 7.05 Tesla and at pressures up to $500 \mathrm{MPa}$ in the temperature range from $220 \mathrm{~K}$ to $420 \mathrm{~K}$. Details of the RF leadthrough are given in the reference. The sample is contained in an $8 \mathrm{~mm}$ NMR-tube and separated from the pressure medium by a sliding $O$-ring seal.

In Fig. 2 the autoclave used in Merbach's group ${ }^{(13,19)}$ is presented. The pressure range of this vessel is $200 \mathrm{MPa}$ and it has been in use for fields up to 9.4 Tesla at temperatures between $250 \mathrm{~K}$ and $400 \mathrm{~K}$. The main emphasis of this construction is on obtaining high sensitivity and high resolution. A resolution $\leqslant 1 \mathrm{~Hz}$ is obtained routinely. The NMR sample is placed inside a $5 \mathrm{~mm}$ NMR tube, the double seal being provided by a neatly finished Macor piston and a cap from the same material.

The construction used in our group is given in Fig. $3 .^{(20,21)}$ It is machined from a high strength titanium alloy and the main new feature is the adaptation of the french double cone seal, ${ }^{(22,23)}$ which in our case, is machined from partially stabilized zirconium dioxide. With this seal, a real low loss RF-leadthrough is provided allowing for rather short $90^{\circ}$ and $180^{\circ}-\mathrm{RF}$ pulses. In normal applications a linewidth of $\sim 2 \mathrm{~Hz}$ is obtainable. The goal of this design was to enable measurements to be made over a large temperature and pressure range, and on a reliable determination of self diffusion

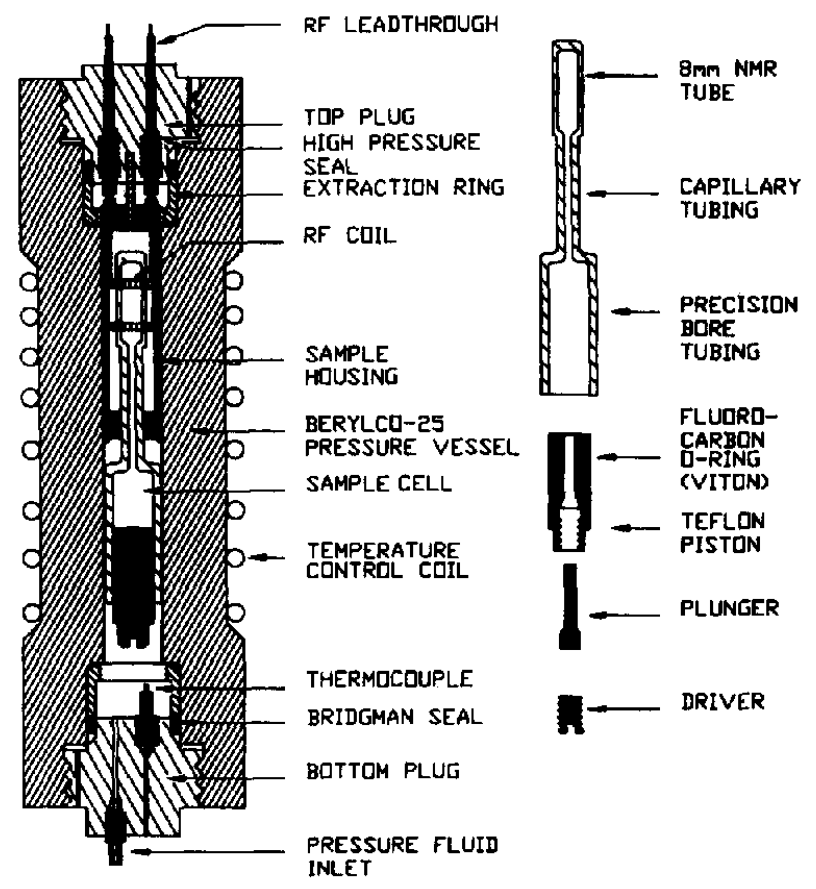

FIG. 1. High pressure HR-NMR probe of Jonas et al. ${ }^{(11)} P_{\max }: 500 \mathrm{MPa} ; T: 220 \mathrm{~K}<T<424 \mathrm{~K}$. 

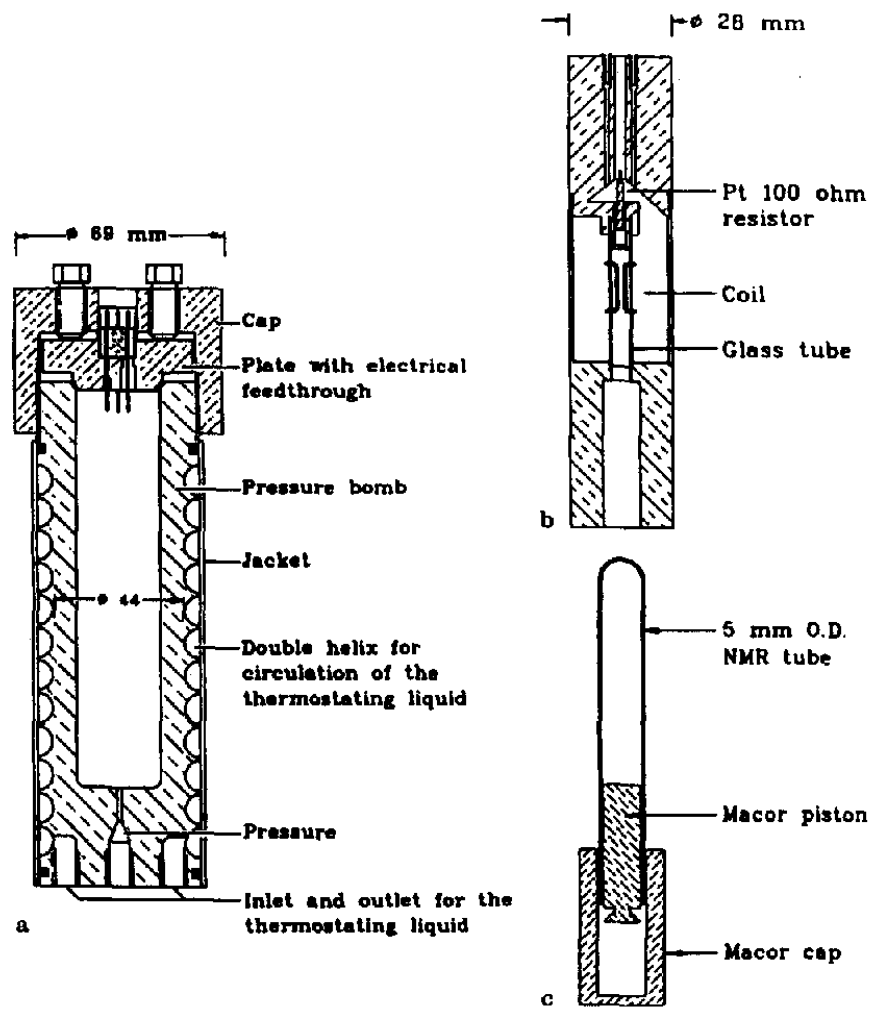

Fig. 2. HR-NMR autoclave designed by Merbach et al. ${ }^{(13.19)} P_{\max }: 200 \mathrm{MPa} ; T: 250 \mathrm{~K}<T<400 \mathrm{~K}$.
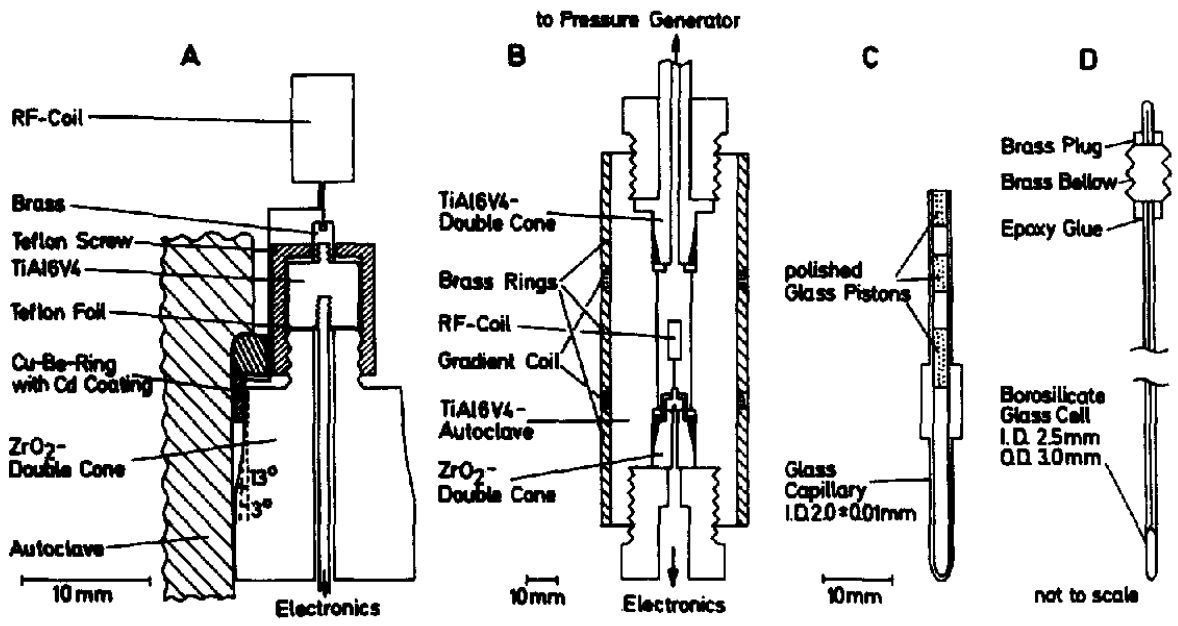

FIG. 3. Autoclave with novel RF-feedthrough for self-diffusion and HR-NMR studies. Bachl et al., (20) Vardag et al. (21) $P_{\max }: 600 \mathrm{MPa} ; T: 150 \mathrm{~K}<T<480 \mathrm{~K}$.

coefficients, measured by the pulsed field gradient technique, ${ }^{(24)}$ with the gradient coil at the outside of the autoclave, in order to avoid a pressure dependent coil geometry. The apparatus has been operated successfully at pressures up to $600 \mathrm{MPa}$ in a temperature range between $150 \mathrm{~K}$ and $500 \mathrm{~K}$. Volatile samples are contained in a borosilicate glass cell sealed with a brass bellow. Samples with higher melting points are filled into a precision glass capillary closed by a series of polished glass pistons. 


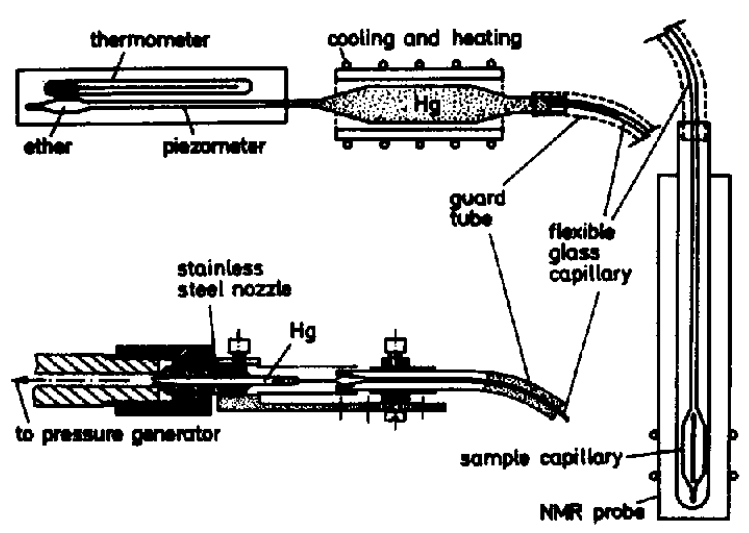

Fig. 4. Yamada's original strengthened glass cell design. ${ }^{(4,16)} P_{\max }: 300 \mathrm{MPa} ; T: 250 \mathrm{~K}<T<350 \mathrm{~K}$.

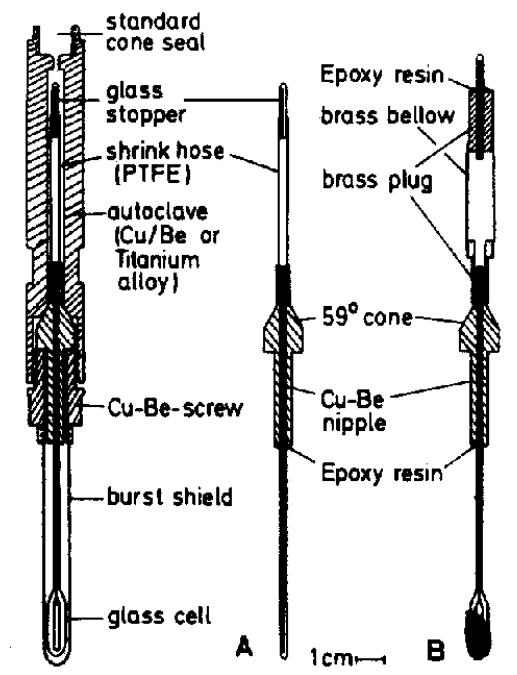

FIG. 5. Strengthened glass cell with conventional pressure generator. ${ }^{(23)} T: 150 \mathrm{~K}<T<480 \mathrm{~K}$. Design A: $P_{\max } 450 \mathrm{MPa}$; design B: $P_{\max } 300 \mathrm{MPa}$.

The original strengthened glass cell design ${ }^{(4,16)}$ is shown in Fig. 4. Typical capillaries have an o.d./i.d. ratio around 4 . In order to remove scratches from both glass surfaces, that might cause breakage of the glass under application of pressure, the cells are etched with dilute aqueous hydrofluoric acid.

The pressure is either generated by heating a closed volume filled with an organic liquid or produced by a conventional high pressure apparatus. Maximum pressures of around $200 \mathrm{MPa}$ are obtained routinely in this design. Occasionally pressures around $300 \mathrm{MPa}^{(25)}$ and up to $400 \mathrm{MPa}^{(26)}$ have been applied, although at a higher breakage rate. Our versions of this type of high pressure apparatus, used routinely over the last decade, are given in Fig. 5. Normally, a teflon shrink hose separates the sample from the pressure transmitting liquid. For gaseous samples, that have to be condensed into the cell by cooling with cold gaseous nitrogen a brass bellow serves as a flexible pressure transmitting and separating system. This system has been used for studying a series of halomethanes in the temperature range of $150 \mathrm{~K}$ to $500 \mathrm{~K}$. For supercooling neat liquids a very thin 

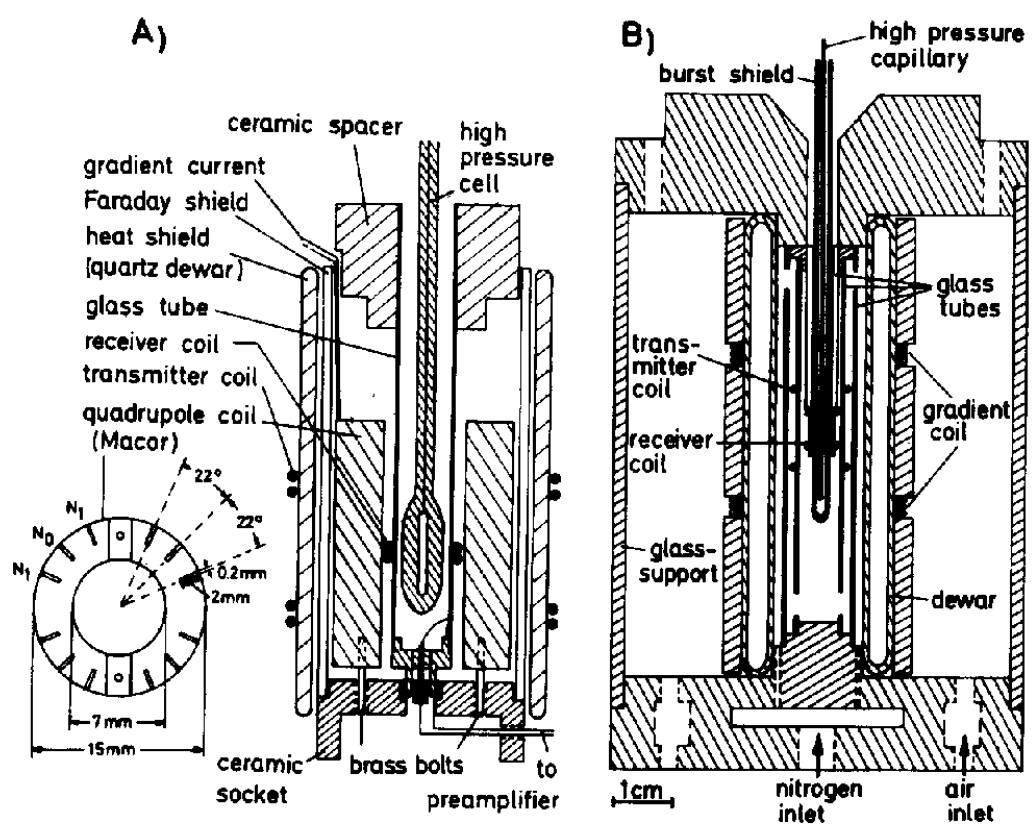

FIG. 6. Cross sections of the modified probe heads. (A) 15" Varian electromagnet. (B) $70 \mathrm{~mm} 7.0$ Tesia Bruker cryomagnet.

capillary (Fig. 5) has been used. These capillaries have an inner diameter between 50 and $200 \mu \mathrm{m}$ and pressures of $450 \mathrm{MPa}$ have been repeatedly obtained. Figure 6 gives the complete modified probe heads used for the determination of self diffusion coefficients employing either electro- or cryomagnets. The design shown in Fig. 6 has been used in Bruker MSL 300 and 100 instruments. In this construction the gradient coil is kept at ambient temperature and pressure, thus permitting the coil constant to be determined with the highest precision possible.

\section{THEORY}

\subsection{Self-Diffusion Coefficients}

3.1.1. Self-Diffusion and the Spin-Echo Experiment. The self-diffusion coefficient in the liquid state is the most fundamental quantity in understanding transport phenomena at the molecular level, and its temperature and pressure dependence is of practical importance. ${ }^{(27)}$ The most convenient way of measuring self-diffusion coefficients in condensed matter is by using the NMR spin-echo experiment. There are several comprehensive reviews of this technique available in the literature. ${ }^{(28,29)}$ The subject has also been reviewed by Weingärtner. ${ }^{(30)}$

Carr and Purcell(31) developed the fixed field gradient spin-echo method suggested by $\mathrm{Hahn}^{(32)}$ and showed that by applying a linear constant magnetic field gradient and monitoring the time decay of the NMR spin-echo amplitude, molecular diffusion could be followed by 'labelling' molecules by means of their nuclear magnetic moments. For the $90^{\circ}-\tau-180^{\circ}$ spin-echo fixed field gradient NMR diffusion measurement, the amplitude of the echo maximum $A$ is given by:

$$
A(2 \tau)=A_{\mathrm{o}} \exp \left[-2 \tau / T_{2}\right] \exp \left[-(2 / 3)(\gamma g \tau)^{2} D \tau\right],
$$

where the term $g=k I$ refers to a constant linear field gradient, $k$ is a calibration factor, $I$ is the coil current, $T_{2}$ is the spin-spin relaxation time, $\tau$ is the time between the $90^{\circ}$ and $180^{\circ}$ pulses and $D$ is the self-diffusion coefficient. The determination of the diffusion coefficient by this method presupposes 
a firm knowledge of the linear field gradient $g$ which is usually calibrated with a substance of precisely known diffusion coefficient and controlled with a second substance with significantly different $T_{2}$ and $D$.

This procedure is often inconvenient and introduces additional errors in high pressure NMR experiments. To overcome this difficulty Lamb et al. ${ }^{(33)}$ developed a method where the linear field gradient strength $g$ and the diffusion coefficient $D$ are determined by using Bessel function fits to the spin-echo signal shape given by:

$$
A(t-2 \tau)=2 A(2 \tau)\left[J_{1}(\gamma \operatorname{gr}(t-2 \tau)) / \gamma g r(t-2 \tau)\right],
$$

where $J_{1}(x)$ is a first order Bessel function and $r$ is the radius of the cylindrical sample.

The most widely used pulsed magnetic field gradient technique for measuring diffusion coefficients is that introduced by Stejskal and Tanner. ${ }^{(34)}$ The determination of $D$ is mostly accomplished by varying the coil current $I$ while keeping all other variables constant. As with the fixed field gradient method the calibration factor $k$ must be determined with a substance whose diffusion coefficient is known precisely, ${ }^{(35)}$ and should always be controlled by testing a second liquid.

In the presence of a pulsed field gradient, the decay of the echo amplitude $A$ in a Hahn spin-echo experiment is given by: ${ }^{(34)}$

$$
A(2 \tau)=A(0) \exp \left[-2 \tau / T_{2}\right] \exp \left[-(\gamma \delta g)^{2}(\Delta-\delta / 3) D\right]
$$

where $\tau$ measures the time between the $90^{\circ}$ and the $180^{\circ}$ pulse, $\gamma$ is the gyromagnetic ratio of the nucleus studied, $\delta$ is the duration of the gradient pulses, $\Delta$ is the time between the two gradient pulses, $g=k I$ is the amplitude of the magnetic field gradient, and $D$ is the self-diffusion coefficient of the nuclear species under investigation. If the nucleus resides in a molecule, and the corresponding atomic species is not exchanged during the time scale of the experiment $(\Delta-\delta / 3)$, the self-diffusion coefficient $D$ reflects the positional fluctuations of the whole molecule. Equation 3 is valid in the limit of sufficiently strong gradients $g \gg\left\langle g_{0}^{2}\right\rangle^{1 / 2}(\tau / \delta)$ compared to any residual homogeneous and stationary field gradients $g_{0}$. Methods to overcome this limitation have been discussed elsewhere. ${ }^{(30,36)}$ As is evident from eqns (1-3) the basic restriction for applying this technique is given by the competing effect of the spin-spin $\left(T_{2}\right)$ relaxation. Hence only nuclei with comparatively long relaxation times $\left(T_{2}\right)$ within the $T, p$-range studied and with sufficiently high gyromagnetic ratios $\gamma$ are accessible to diffusion measurements made with the NMR method.

3.1.2. Self-Diffusion Coefficients in Hard Sphere Fluids. In the absence of any satisfactory analytical theory of transport coefficients in dense fluids, the hard sphere fluid may establish a suitable reference system upon which more realistic models may be based. ${ }^{(27,37,38)}$ It is well known that short-ranged repulsive interactions dominate the dynamic structure of simple molecular liquids. Modern perturbation theories of liquids successfully relate their thermodynamic and dynamic properties to those of a purely repulsive system. ${ }^{(39,40)}$ This approach can be extended to include the retarding effect of weakly attractive interactions upon single particle dynamics. The resulting expression for the diffusion coefficient $D(\rho, T)$ represents the density and temperature dependence of translational motions rather successfully for liquids without strong, anisotropic attractive interactions like hydrogen bonds. The Chapman-Enskog solution of the Boltzmann equation gives the diffusion coefficient $D_{E}$ for moderately dense gases of hard spheres

$$
D_{\mathrm{E}}=\frac{3}{8}\left(\frac{k T}{\pi m}\right)^{1 / 2}\left(\frac{\sigma}{\rho}\right) g^{-1}(\sigma)=\frac{D_{0}}{\rho g(\sigma)},
$$

where $\sigma$ is the hard sphere diameter, $\rho=n \sigma^{3}$ the packing fraction or reduced density, $n$ is the number density, $m$ the molecular mass and $g(\sigma)$ the radial distribution function of the hard spheres at contact. $g(\sigma)$ may be evaluated quite accurately from the Carnahan-Starling approximation. ${ }^{(41)}$

$$
g(\sigma)=\left(\frac{p}{n k T}-1\right) / \rho b=\frac{1}{2}(2-\eta) /(1-\eta)^{3},
$$


with $\eta=\pi / 6 n \sigma^{3}=\pi / 6 \rho$ and $b=2 \pi / 3 \sigma^{3}$ or from a recently derived accurate equation of state of the hard sphere fluid ${ }^{(42)}$ yielding:

$$
g(\sigma)=2.64 /(\rho-1.625)^{2} .
$$

At higher densities correlated motions and back scattering events necessitate a modification of the Chapman-Enskog expression according to:

$$
D_{\mathrm{HS}}=D_{\mathrm{o}} \frac{P(\rho)}{\rho},
$$

where $g(\sigma)$ is replaced by $P(\rho)$, an empirical polynomial in the packing fraction $\rho$. The coefficients of $P(\rho)$ may be deduced from computer simulations of the hard sphere fluid. A recent reassesment ${ }^{(43)}$ of the most extensive computer simulations of the hard sphere fluid yielded:

$$
D_{\mathrm{HS}}=D_{\mathrm{o}} \frac{P(\rho)}{\rho}=\frac{D_{\mathrm{o}}}{\rho}\left(1-\frac{\rho}{1.09}\right)\left(1+0.4 \rho^{2}-0.83 \rho^{4}\right) \text {. }
$$

3.1.3. Self-diffusion Coefficients in Simple, Non-Associated Liquids. In most real molecular liquids with weak isotropic interactions, eqn (8) represents the density dependence of the self-diffusion coefficient $D$ very well, but the absolute value of $D$ is considerably overestimated. In real fluids a reduction of the molecular diffusivity may result from two different mechanisms. Deviations of the molecular shape from spherical symmetry may produce a coupling of rotational and translational motions during molecular collisions resulting in a reduced self-diffusion coefficient. Chandler ${ }^{(44)}$ showed that rotation-translation coupling can be accounted for via

$$
D=A D_{\mathrm{HS}}(T, \rho) \text {. }
$$

The coupling factor $A$, where $0<A \leqslant 1$, is rigorously independent of the number density. Furthermore it is expected to depend on temperature only weakly if at all.

Attractive molecular interactions may also cause a retardation of translational single particle motions. Speedy et al. ${ }^{(45)}$ were able to incorporate the effect to weak isotropic attractive interactions into the hard sphere treatment of diffusion. Representing the attractive interactions by a LennardJones potential, and accounting for a dependence of the effective diameter $\sigma$ upon the kinetic energy during collisions in a self-consistent manner, the diameter $\sigma$ becomes temperature dependent according to:

$$
\sigma_{\mathrm{B}}(T)=\sigma_{\mathrm{LJ}} 2^{1 / 2}\left[1-\left(1+2 k T / \varepsilon_{\mathrm{L}}\right)^{1 / 2}\right]^{1 / 6}
$$

Alternative expressions for an effective, temperature dependent diameter $\sigma(T)$ in connection with the Lennard-Jones fluid have been proposed ${ }^{(39,46-48)}$ and have been discussed thoroughly by Heyes et al. (37. 38) $^{3}$

The resulting self-diffusion coefficient $D$ then depends on temperature and number density via

$$
D_{\mathrm{LJ}}(T, \rho)=D_{\mathrm{HS}}\left(T, \rho, \sigma_{\mathrm{B}}\right) \exp \left(-\varepsilon_{\mathrm{LJ}} / 2 k T\right) \text {. }
$$

In actual applications of eqn (11) to interpret experimental self-diffusion coefficients of simple liquids the Lennard-Jones diameter $\sigma_{\mathrm{LJ}}$ and the potential depth $\varepsilon_{\mathrm{L}}$ have to be adjusted in keeping with estimates of these parameters from independent physical quantities. This places severe restrictions upon any free adjustment of these parameters.

Heyes et al ${ }^{(37.38)}$ also discussed the conventional representation of the temperature and pressure dependence of transport coefficients based upon the activation model

$$
D^{-1}(T, p)=D_{0}^{-1} \exp \left[\left(E^{*}+p V^{*}\right) / k T\right],
$$

with $E^{*}$ an apparent activation energy and $V^{*}$ an activation volume. The apparent activation energy depends on whether the volume or pressure is maintained constant:

$$
\begin{aligned}
& E_{\mathrm{v}}^{*}=\left[\partial \ln D^{-1} / \partial(1 / k T)\right]_{\mathrm{v}} \\
& E_{\mathrm{p}}^{*}=\left[\partial \ln D^{-1} / \partial(1 / k T)\right]_{\mathrm{p}} .
\end{aligned}
$$


Also by definition

$$
V^{*}=k T\left[\partial \ln D^{-1} / \partial p\right]_{T},
$$

and

$$
E_{\mathrm{v}}^{*}=E_{\mathrm{p}}^{*}-T V^{*}(\partial p / \partial T)_{\mathrm{v}}=E_{\mathrm{p}}^{*}-T(\beta / \kappa) V^{*} .
$$

Both apparent activation energies are related to the thermal expansivity $\beta$ of the fluid and the close-packed volume expansivity $\beta_{0}$ via:

$$
\begin{gathered}
E_{\mathrm{V}}^{*} / k T=\frac{1}{2}+\beta_{\mathrm{o}}\left[1+\frac{1}{\rho}\left(\frac{\partial \ln P(\rho)}{\partial(1 / \rho)}\right)_{\mathrm{T}}\right] ; \\
E_{\mathrm{p}}^{*} / k T=\frac{1}{2}+\left(\beta_{\mathrm{o}}-\beta\right)\left[1+\frac{1}{\rho}\left(\frac{\partial \ln P(\rho)}{\partial(1 / \rho)}\right)_{\mathrm{T}}\right],
\end{gathered}
$$

with $\beta=V^{-1}(\partial V / \partial T)_{0}$ and $\beta_{0}=3 \sigma^{-1}(\partial \sigma / \partial T)_{b}$.

In non-associated liquids $E_{\mathrm{p}}^{*}$ is almost constant with rising pressure at constant temperature, $E_{v}^{*}$ is about half the value of $E_{\mathrm{p}}^{*}$, and the ratio $E_{\mathrm{v}}^{*} / E_{\mathrm{p}}^{*}$ increases with density. In associated liquids $E_{\mathrm{v}}^{*}$ and $E_{\mathrm{p}}^{*}$ become nearly equal. Similarly the activation volume $V^{*}$ is related to the isothermal compressibility $\kappa$ of the liquid via

$$
V^{*} / k T=\kappa\left[1+\rho^{-1}(\partial \ln P(\rho) / \partial(1 / \rho))_{T}\right],
$$

with $\kappa=-(\partial V / \partial p)_{\mathrm{T}} / V$ and $P(\rho)$ the appropriate empirical polynomial used to represent the hard sphere fluid. Explicit expressions have been given for the Enskog model and the polynomial $P(\rho)$ obtained by Dymond. ${ }^{(46,47)}$ The activation model has been questioned by computer simulations, ${ }^{(49,30)}$ which show that molecular motions do not proceed by activated jumps whose lengths are approximately equal to the intermolecular spacing as implied by the activation model, but by a succession of small diffusive steps. Further empirical relations $D(T, p)$ are discussed by Collings and Woolf, ${ }^{(51)}$ Saeki et al., ${ }^{(52)}$ Harris et al., ${ }^{(53)}$ and Polzin and Weiss. ${ }^{(54)}$

Instead of mapping experimental temperature and pressure dependencies of transport coefficients onto a set of apparent activation parameters, free volume considerations are often applied. Doolittle $^{(55)}$ and later Cohen and Turnbull ${ }^{(56)}$ considered the diffusion process as being driven by density fluctuations relating transport coefficients to a mean free volume per particle $\left\langle v_{\mathrm{f}}\right\rangle$ and obtained for the self-diffusion coefficient

$$
D=\frac{1}{3}\left(\frac{3 R T}{M}\right)^{1 / 2} \frac{4}{\pi \sigma^{2}}\left(v_{f}^{*}+\left\langle v_{f}\right\rangle\right) \exp \left(-v_{f}^{*} /\left\langle v_{f}\right\rangle\right)
$$

where $\sigma$ is the hard sphere diameter, $\left\langle v_{f}\right\rangle\left(=\langle v\rangle-v_{o}\right)$ is the mean free volume of the molecule with $\langle v\rangle\left(=M /\left(\rho N_{\mathrm{A}}\right)\right)$ the mean volume of the molecule and $v_{0}=(4 \pi / 3) \sigma^{3}$ the van der Waals volume. $v_{f}^{*}=2 v_{0}$ is the critical free volume of the molecule that must be provided for fluid flow to occur. Hence at constant density, the self-diffusion coefficient is proportional to $D \sim T^{1 / 2}$, a prediction not always met by real fluids. ${ }^{(37)}$ Furthermore, in associated liquids, such as water, the diffusion coefficient varies considerably without a significant change in density. This shows that other properties besides free volume need to be considered in modelling the diffusion process.

3.1.4. Self-Diffusion Coefficients in H-Bonded Liquids. In liquids with anisotropic and saturable H-bonding interactions, isobaric transport coefficients vary much stronger with temperature than is predicted by the formalism discussed in Sections 3.1.2. and 3.1.3. The deviations are most pronounced in the random, transient $\mathrm{H}$-bond network of liquid water. ${ }^{(58-60)}$ Even more striking is the density dependence of both translational and rotational diffusive motions which show highly anomalous behaviour in undercooled liquid water. The latter can be related to some structural contributions from the strongly directional hydrogen bonding interactions and their dynamics within the network.

In spite of there being many structural models of liquid water, only a few attempts have been undertaken to develop a theory of translational and rotational diffusion involving $\mathbf{H}$-bond dy- 
namics. ${ }^{(61-72)}$ Sposito ${ }^{(67,68)}$ explored the long-time behaviour of diffusional properties within the context of a hydrodynamic approach. Translational $D_{\mathrm{s}}$ and rotational $D_{\mathrm{r}}$ diffusion coefficients are related to the shear viscosity $\eta$ and a factor $\beta$, specifying the boundary condition at the sphere-liquid interface ${ }^{(73)}$ as shown below:

$$
\begin{gathered}
D_{\mathrm{s}}=\frac{k T}{\xi_{\mathrm{t}}(\omega=0)}=\frac{k T}{4 \pi \eta r} \frac{1+f}{1+(3 / 2) f}= \begin{cases}k T / 6 \pi \eta r, & \beta \rightarrow \infty \\
k T / 4 \pi \eta r, & \beta \rightarrow 0\end{cases} \\
D_{\mathrm{r}}=\frac{k T}{\xi_{\mathrm{r}}(\omega=0)}=\frac{k T}{8 \pi r^{3}} \frac{1+f}{f}= \begin{cases}k T / 8 \pi \eta r^{3}, & \beta \rightarrow \infty \\
\infty, & \beta \rightarrow 0\end{cases} \\
f=\beta r / 3 \eta .
\end{gathered}
$$

When $\beta$ becomes large the liquid sticks at the surface of the sphere and eqns (21) and (22) reduce to the well-known Stokes-Einstein and Debye-Stokes relations, respectively. For vanishingly small $\beta$ the friction coefficient $\xi_{t}(\omega=0)=4 \pi \eta r$ corresponds to perfect slip conditions. The rotational diffusion coefficient $D_{\mathrm{r}}$, however, diverges in this limit, indicating a complete decoupling of rotational and translational motions. Zwanzig ${ }^{(74)}$ proposed another relation between $D$ and $\eta$ based on configuration space considerations thereby stressing the importance of the hopping rate as an essential element in a novel theory of self-diffusion. With $v=V / N$ the volume per particle his relation reads:

$$
D(\eta / k T) v^{1 / 3}=C, \quad 0.132 \leqslant C \leqslant 0.181 .
$$

An empirical modification of the Stokes-Einstein relation in the form ${ }^{(75,417)}$

$$
D \eta^{\alpha}=\text { constant }, \quad \alpha \neq 1
$$

has also been used occasionally with $\alpha$ a non-universal exponent which varies with temperature and the material considered. However, Z $\mathrm{Z}_{\text {wanzig and Harrison }}{ }^{(76)}$ argue in favour of the standard Stokes-Einstein relation but with an effective hydrodynamic radius of the moving particle.

These mode-coupling expressions interrelate different transport coefficients, but they do not provide any explicit dependence on temperature and density nor do they consider the role of $\mathrm{H}$-bond interactions and their fluctuations. Several authors have treated the long-time diffusional properties involving the $\mathrm{H}$-bond dynamics within a two-state (on, off) approximation of the $\mathrm{H}$-bond interaction.

In his bond lattice approach, Angell ${ }^{(61)}$ considered a totally connected random tetrahedral network structure as the configurational ground state which would be reached at $T_{0}$. Temperature and pressure cause excitations in the bond lattice with the equilibrium number of 'off'-elements $n_{\text {off }}(T, p)$ given by standard lattice statistics. Translational motions follow from statistical fluctuations in the local concentrations of 'off'-elements as $n_{\mathrm{off}}(T, p)$ increases with $T$ and $p$. Any cooperativity between H-bonds has been neglected.

The probability $p(r)$ of cooperative particle rearrangements was postulated to be an exponential function of the broken bond fraction $n_{\text {orr }}$, which in turn is an almost linear function of temperature and extrapolates to zero at $T_{0}$, i.e. $n_{\mathrm{orf}}(T, p) \sim\left(T-T_{0}(p)\right)$. The self-diffusion coefficient was obtained as:

$$
D \sim \frac{1}{\tau} \sim \exp \left(-\frac{1}{n_{\text {orr }}(T, p)}\right) \sim \exp \left(\frac{T_{0}(p)}{T-T_{\mathrm{o}}(p)}\right),
$$

with $\tau$ a microscopic time constant characteristic for structural rearrangements within the network. The resulting VTF-form ${ }^{(77-79)}$ is widely used in complex liquids to represent the isobaric temperature dependence of transport and relaxation coefficients.

Stanley and Teixeira ${ }^{(62)}$ further explored the statistical consequences of the bond connectivity aspect of the $\mathrm{H}$-bond network within a correlated bond percolation model.

Bertolini et al. ${ }^{(63-65)}$ recently pursued this approach further and developed an exhaustive theory of long-time diffusional events based on $\mathrm{H}$-bond dynamics. Water molecules were classified according to the number of intact $\mathrm{H}$-bonds via a discrete stochastic variable $\left|\eta_{j}\right\rangle,(j=0, \ldots, 4)$. H-bonds were 
assumed to be randomly intact with bond probability $p_{\mathrm{B}}$. The fraction $p_{j}$ of water molecules with $j=0, \ldots, 4$ intact bonds was given simply by the binomial distribution

$$
p_{f}(t \rightarrow \infty)=\left(\begin{array}{l}
4 \\
j
\end{array}\right) p_{\mathrm{B}}^{j}\left(1-p_{\mathrm{B}}\right)^{4-j}
$$

but their connectivity was far from random. ${ }^{(79)}$ Molecules with four intact bonds tended to clump together into patches of high local order. The bond probability $p_{\mathrm{B}}(T, p)$ increased linearly with decreasing temperature causing drastic changes in the network topology as the fraction $p_{4}$ of molecules with four intact bonds is given by:

$$
p_{4}(T, p)=p_{B}^{4}(T, p) .
$$

Although no explicit pressure or density dependence $p_{\mathrm{B}}(p)$ was given, $p_{\mathrm{B}}(T, p)$ was also supposed to decrease with increasing pressure. Transport properties were calculated within a generalized itinerant oscillator model supplemented with a master equation for the time-dependent populations $p_{j}(t)$ to account for the finite life-times of the H-bonds and the inherently transient nature of the network. The self-diffusion coefficient $D$ was obtained as the mean over diffusion coefficients $D\left(\eta_{j}\right)$ of the states $\left|\eta_{\mathrm{j}}\right\rangle$, weighted according to the corresponding equilibrium distribution:

$$
D=\sum_{j=0}^{4} D\left(\eta_{j}\right) p_{j}(t \rightarrow \infty)
$$

The model can be reformulated exactly in terms of a two-state description. Transport properties may then be regarded as arising from the mobile fraction of water molecules with two H-bonds at most, i.e. $D\left(\eta_{j}\right)=0$ for $j=3,4$.

\subsection{Spin-Lattice $\left(T_{1}\right)$ and Spin-Spin $\left(T_{2}\right)$ Relaxation}

3.2.1. Relaxation Theory. In 1946 Bloch $^{(80)}$ introduced the concept of relaxation times to nuclear magnetic resonance phenomena. He assumed that the relaxation of a spin system in a strong static field $B_{0}$ after a small perturbation can be characterized by two relaxation rates, the longitudinal or spin-lattice rate $R_{1}=1 / T_{1}$ and the transverse or spin-spin relaxation rate $R_{2}=1 / T_{2}$. During the spin-lattice relaxation process energy is transfered from the spin system to the lattice whereas the spin-spin relaxation process effects a redistribution of the energy within the spin system. A microscopic theory of nuclear spin relaxation was presented shortly afterwards by Bloembergen, Purcell and Pound ${ }^{(81)}$ and some faults contained in their paper were corrected later by Solomon. ${ }^{(82)}$ The whole field quickly developed into a versatile and powerful spectroscopic method as several excellent textbooks demonstrate. ${ }^{(83-86)}$ Also Kowalewski ${ }^{\left({ }^{(97.88)}\right.}$ has recently presented a clear and comprehensive review of the nuclear spin relaxation in diamagnetic liquids. The basic ideas of the BPP theory were developed into a complete theoretical framework by Wangsness, Bloch and Redfield. ${ }^{\text {(89-92) }}{ }^{\text {The }}$ WBR or Redfield theory is given in terms of a density matrix rather than dealing with magnetizations or energy level populations. The density matrix $\rho$ describes the average behaviour of an ensemble of quantum mechanical systems and allows one to calculate any observable $\langle Q\rangle$ via $^{(83-85)}$

$$
\langle Q\rangle=\operatorname{Tr}(\rho \bar{Q})
$$

by taking the trace of the density matrix and the matrix representation of the corresponding operator $\hat{Q}$ in the same basis set. A knowledge of the time evolution of the density matrix is then sufficient to describe any experiment on a macroscopic system.

The essence of the WBR theory is the division of the total system into a spin system comprising the relevant spin-dependent degrees of freedom, and a lattice representing the remaining degrees of freedom.

The coupling between the two subsystems is assumed to be weak so that the total spin Hamiltonian may be written as: ${ }^{(84)}$

$$
\hat{H}=\hat{H}_{\mathrm{o}}+\hat{H}_{\mathrm{SL}}(t)
$$


where $\hat{H}_{\mathrm{o}}$ contains all time-independent interactions (Zeeman, indirect spin-spin coupling etc.) and $H_{\text {SL }}(t)$ represents the time dependent spin-lattice coupling giving rise to relaxation. These couplings can be written as a product of irreducible tensor operators ${ }^{(83)}$

$$
\hat{H}_{\mathrm{SL}}=\sum_{m}(-1)^{m} A_{\mathrm{m}}^{(L)} T_{\mathrm{m}}^{(\mathrm{L})}
$$

where $A_{m}^{(L)}$ is the $m$-component of a tensor operator of rank $L$ which involves only spin coordinates and $T_{m}^{(L)}$ is a tensor operator containing only lattice variables. If the motion in the lattice, characterised by some correlation time $\tau_{c}$, is fast compared to the strength of the interaction $\left|\left\langle H_{\text {SL }}\right\rangle\right|$ (strong narrowing condition) where

$$
\left|\left\langle\hat{H}_{\text {SL }}\right\rangle\right| \tau_{\mathrm{c}} \ll 1,
$$

then the elements of the density matrix obey an equation of motion

$$
\rho(t)=i\left[\rho, \hat{H}_{\mathrm{o}}\right]-\underline{\mathrm{R}}\left[\rho-\rho_{\mathrm{o}}\right] .
$$

In eqn (33) $\rho_{\mathrm{o}}$ is the density matrix in thermal equilibrium and $R$ is the relaxation matrix with elements

$$
\begin{aligned}
R_{\alpha \alpha^{\prime} \beta \beta^{\prime}}= & \sum_{m}\left[\delta_{\alpha \beta}\left(A_{-m}^{(\mathbf{L})}\right)_{\beta^{\prime} \gamma}\left(A_{m}^{(\mathbf{L})}\right)_{\gamma \alpha^{\prime}} g_{m_{z}-m}^{(\mathbf{L})}\left(\omega_{\beta^{\prime} \gamma}\right)+\sum_{\gamma} \delta_{\alpha^{\prime} \beta^{\prime}}\left(A_{m}^{(\mathbf{L})}\right)_{\alpha \gamma}\left(A_{-m}^{(\mathbf{L})}\right)_{\gamma \beta} g_{m,-m}^{(\mathbf{L})}\left(\omega_{\gamma \beta}\right)\right. \\
& \left.-2\left(A_{-m}^{(\mathbf{L})}\right)_{\alpha \beta}\left(A_{m}^{(\mathbf{L})}\right)_{\beta^{\prime} \alpha^{\prime}} \operatorname{Re}\left\{g_{m,-m}^{(L)}\left(\omega_{\alpha \beta}\right)\right\}\right]
\end{aligned}
$$

where $\alpha, \beta$ and $\gamma$ label eigenstates of $H_{0}$ and $\hbar \omega_{\alpha \beta}=E_{\alpha}-E_{\beta}$.

The spectral density functions are defined as Fourier-Laplace transforms

$$
g_{\mathrm{m},-\mathrm{m}}^{(\mathrm{L})}(\omega)=\int_{0}^{\infty} G_{\mathrm{m},-\mathrm{m}}^{(\mathbf{L})}(\tau) \exp (-i \omega \tau) \mathrm{d} \tau
$$

of the time autocorrelation functions

$$
G_{m,-m}^{(\mathbf{L})}(\tau)=(-1)^{m}\left\langle T_{m}^{(L)}(\tau) T_{-m}^{(\mathbf{L})}(0)\right\rangle
$$

of the fluctuating lattice degrees of freedom. Regarding the time window of NMR relaxation spectroscopy $\left(\sim 2 \pi / \omega_{\mathrm{L}}\right)$ the relevant fluctuations mainly concern molecular rotational and translational diffusion. Faster quasi-lattice vibrations (QLV) mainly average out part of the spin-lattice coupling but do not contribute to the relaxation significantly. ${ }^{(93-97)}$ In simple cases the decay of the corresponding correlation function can be approximated by:

$$
G_{\mathrm{m},-\mathrm{m}}^{(\mathrm{L})}(t)=\langle G(\mathrm{o})\rangle_{\mathrm{QLV}} \exp \left(-t / \tau_{\mathrm{L}}\right)
$$

with \langle\rangle$_{\text {QLv }}$ an average over quasi-lattice vibrations and $\tau_{\mathrm{L}}(T, p)$ a characteristic correlation time of the fluctuations which depend strongly on the thermodynamic state of the system. Exponential correlation functions lead to Lorentzian spectral densities

$$
g_{m,-m}^{(\mathbf{L})}\left(\omega_{L} \tau_{L}\right)=\frac{\tau_{L}}{1+\omega_{L}^{2} \tau_{L}^{2}}
$$

where the Larmor frequency $\omega_{\mathrm{L}}$ corresponds to the energy differences in eqn (34). In the extreme narrowing limit $\omega_{\mathrm{L}}^{2} \tau_{\mathrm{L}}^{2} \ll 1$ the spectral density corresponds to the integral over the correlation function which may be used to define an effective correlation time

$$
\tau_{\mathrm{eff}}=g(0)=\int_{0}^{\infty} G_{m,-m}^{(L)}(t)
$$

irrespective of the form of $G_{m,-m}^{(L)}(t)$.

Equation 29 can be combined with eqn (33) to obtain the time evolution of a certain magnetization component. It is, however, not always possible to define appropriate relaxation times $T_{1}$ and $T_{2}$ except in the extreme narrowing limit. It is mainly in the state dependence of the microscopic time 
constants for diffusive molecular motions where high pressure NMR experiments become useful and lead to new information. By changing independently the temperature and the density during a set of relaxation experiments, energetic and structural aspects of molecular dynamics may be separated.

Recently Szymanski, Gryff-Keller and Binsch ${ }^{(98)}$ presented a rather general formulation of the WBR theory based on the Liouville formalism ${ }^{(\mathbf{9 9}, 85)}$ which is also suitable for computer calculations.

The WBR theory is not valid outside the motional narrowing regime, where the strong narrowing condition holds. This limit is often called the slow motion regime. In this range the equation of motion, eqn (33), has to be replaced by the stochastic Liouville equation [SLE]. This approach is discussed thoroughly by Kubo, ${ }^{(100)}$ Lynden-Bell, ${ }^{(99)}$ Freed et al. ${ }^{(101)}$ and Westlund. ${ }^{(102)}$ It has, however, not yet found applications within the field of high pressure NMR relaxation.

Spin-lattice and spin-spin couplings are mediated by local electric and/or magnetic fields, which fiuctuate in time due to molecular rotations and translations and provide the various relaxation mechanisms, which are discussed next.

3.2.2. Dipole-Dipole (DD) Relaxation. The magnetic coupling between two nuclear magnetic moments associated with nuclear spins $I, S \neq 0$ is by far the most important relaxation mechanism, especially for spin $1 / 2$ nuclei. The relevant interaction Hamiltonian expressed in the laboratory-fixed frame $(\mathrm{L})$ may be written as ${ }^{(103)}$

$$
\hat{H}_{\mathrm{DD}}^{\mathrm{L}}(t)=-2 \gamma_{1} \gamma_{\mathrm{S}} \mathrm{h}\left(\mu_{0} / 4 \pi\right) \sum_{m=-2}^{2}(-1)^{m} A_{m}^{(2)} T_{-m}^{(2)}(t)
$$

where the $A_{m}^{(2)}$ terms involve spin degrees of freedom only. The $T_{m}^{(2)}$ terms contain lattice degrees of freedom which are constants, when expressed in a molecule-fixed principal axis system $(P)$. The transformation to the Lab-frame becomes time-dependent because of molecular tumbling, hence:

$$
T_{m}^{(2)}=\sum_{m^{\prime}=-2}^{2} \rho_{2 m^{\prime}} D_{m,-m}^{(\mathbf{L})}(\Omega(t)) .
$$

$D_{m}^{(L)}(\Omega)$ are components of the Wigner rotation matrix and the principal components of the dipoledipole tensor are:

$$
\rho_{20}=\left(\frac{3}{2}\right)^{1 / 2}\left(\frac{1}{r_{\text {LS }}}\right)^{3}, \quad \rho_{2 \pm 1}=\rho_{2 \pm 2}=0
$$

Expressions for the DD relaxation in a two spin $(I, S)$ system within a molecule were first given by Solomon ${ }^{(82)}$ who obtained:

$$
\begin{aligned}
& \left\langle I_{\mathrm{Z}}\right\rangle=-R_{1 \mathrm{II}}\left(\left\langle I_{\mathrm{Z}}\right\rangle-I_{\mathbf{Z}}^{\circ}\right\rangle-R_{\mathrm{IS}}\left(\left\langle S_{\mathrm{Z}}\right\rangle-S_{\mathrm{Z}}^{\circ}\right) \\
& \left\langle S_{\mathrm{Z}}\right\rangle=-R_{\mathrm{SI}}\left(\left\langle I_{\mathrm{Z}}\right\rangle-I_{\mathrm{Z}}^{\circ}\right)-R_{1 \mathrm{~s}}\left(\left\langle S_{\mathrm{Z}}\right\rangle-S_{\mathbf{Z}}^{\circ}\right)
\end{aligned}
$$

with $I_{Z}^{\circ}$ and $S_{\mathbf{Z}}^{\circ}$ the equilibrium longitudinal magnetizations of the $I$ and $S$ spins respectively, and with the relaxation rates:

$$
\begin{gathered}
R_{1 \mathrm{D}}^{\mathrm{DD}}=\frac{2}{15}\left(\gamma_{1} \gamma_{\mathrm{S}} \mathrm{h}\right)^{2} S(S+1)\left\langle r_{\mathrm{IS}}^{-3}\right\rangle^{2}\left[g\left(\omega_{\mathrm{IS}}^{-} \tau_{2}\right)+3 g\left(\omega_{1} \tau_{2}\right)+6 g\left(\omega_{\mathrm{IS}}^{+} \tau_{2}\right)\right] \\
R_{\mathrm{IS}}^{\mathrm{DD}}=\frac{2}{15}\left(\gamma_{1} \gamma_{\mathrm{S}} \mathrm{h}\right)^{2} S(S+1)\left\langle r_{\mathrm{IS}}^{-3}\right\rangle^{2}\left[6 g\left(\omega_{\mathrm{IS}}^{-} \tau_{2}\right)-g\left(\omega_{\mathrm{IS}}^{+} \tau_{2}\right)\right] .
\end{gathered}
$$

The general solutions for $\left\langle I_{z}\right\rangle$ and $\left\langle S_{z}\right\rangle$ are sums of two exponentials, hence the decay of the longitudinal magnetizations in a two-spin system do not follow the Bloch equations. Simple exponential behaviour is recovered, however, under the following conditions (i) the two spins are identical, (ii) the $S$ spins have an additional strong relaxation mechanism, hence remain practically in thermal equilibrium during $I$ spin relaxation, (iii) the $S$ spin system is saturated by an intense $r f$ field. The decay 
of the transverse magnetization components follow Bloch's equation with a relaxation rate given by:

$$
R_{2}^{\mathrm{DD}}=\frac{1}{15}\left(\gamma_{1} \gamma_{\mathrm{S}} \mathrm{h} \mu_{\mathrm{o}} / 4 \pi\right)^{2} S(S+1)\left\langle r_{\mathrm{IS}}^{-3}\right\rangle^{2}\left[4 \tau_{2}+g\left(\omega_{\mathrm{IS}}^{-} \tau_{2}\right)+6 g\left(\omega_{\mathrm{S}} \tau_{2}\right)+3 \mathrm{~g}\left(\omega_{1} \tau_{2}\right)+6 g\left(\omega_{\mathrm{IS}}^{+} \tau_{2}\right)\right]
$$

with $\omega_{\mathrm{Is}}^{-}=\omega_{1}-\omega_{\mathrm{s}}, \quad \omega_{\mathrm{IS}}^{+}=\omega_{1}+\omega_{\mathrm{s}}$.

Representing the molecular tumbling motions by a Brownian rotational diffusion model with a single correlation time for each component results in Lorentzian spectral densities (eqn 38). If the two coupled spins reside on different molecules, the relative distance $r_{\text {Is }}$ of both nuclei becomes time-dependent also. The dipole-dipole interaction is then modulated by translational and rotational motions. ${ }^{(104-106)}$ The corresponding intermolecular spin-lattice relaxation rate may be obtained by inserting into eqn (45) the spectral density function appropriate to a force-free diffusion model with reflecting boundary conditions as ${ }^{(107,108)}$

$$
R_{1, \text { inter }}^{\text {DD }}=\frac{8 \pi}{15}\left(\gamma_{1} \gamma_{\mathrm{s}} h\right)^{2} S(S+1)\left[g\left(\omega_{\mathrm{IS}}^{-} \tau_{\mathrm{d}}\right)+3 g\left(\omega_{1} \tau_{\mathrm{d}}\right)+6 g\left(\omega_{1 \mathrm{~S}}^{+} \tau_{\mathrm{d}}\right)\right]
$$

with

$$
g(u)=\left(N_{\mathrm{s}} \tau_{\mathrm{d}} / d_{\mathrm{sS}}^{3}\right)\left[\left(\frac{3}{2} u^{2}+\frac{15}{2} u+12\right) /\left(\frac{1}{8} u^{6}+u^{5}+4 u^{4}+\frac{27}{2} u^{3}+\frac{81}{2} u^{2}+81 u+81\right)\right]
$$

whereby

$$
\begin{gathered}
u^{2}=2 \omega \tau_{\mathrm{d}} \text { and } \tau_{\mathrm{d}}=d_{\mathrm{IS}}^{2} / D_{\mathrm{rel}}(I-S) \\
D_{\mathrm{rel}}(I-S)=D(I)+D(S)
\end{gathered}
$$

$d_{\text {IS }}$ is the distance of closest approach of the spins, $D(I)$ and $D(S)$ are the self-diffusion coefficients of the spin bearing particles and $N_{\mathrm{S}}$ is the number density of spins $S$.

Werbelow ${ }^{(109,110)}$ further noted that for coupled nuclei with magnetogyric ratios of opposite signs, as for ${ }^{1} \mathrm{H}-{ }^{17} \mathrm{O}$ for example, care has to be taken with respect to the relative signs of the Larmor frequencies. In dipolar coupled spin systems with more than two spins and spin systems with indirect spin-spin coupling the longitudinal relaxation follows a set of coupled differential equations. ${ }^{(111-113)}$ These equations simplify under decoupling conditions but the latter do not necessarily lead to single exponential relaxation processes in spin systems like ${ }^{13} \mathrm{CH}_{2}$ or ${ }^{13} \mathrm{CH}_{3}$ groups. Also cross-correlation of different dipolar interactions may occur. ${ }^{(114-116)}$ Outside the extreme narrowing limit, the longitudinal relaxation in a multipolar $\mathrm{AX}$ system $\left(S_{\mathrm{A}} \geqslant 1\right.$ and $\left.I_{\mathrm{X}}=1 / 2\right)$ can differ considerably from either an isolated dipolar coupled pair or from an isolated quadrupolar nucleus. ${ }^{(117-120)}$ However, this complexity is effectively suppressed whenever spin A is relaxed in a time short compared to the relaxation of spins $X$. In practice, this limit is often obtained since spin $A$ is susceptible to the highly effective quadrupolar relaxation mechanism.

3.2.3. Quadrupolar Relaxation. The relaxation of nuclei with a spin larger than $1 / 2$ is dominated by a coupling of the electric quadrupole moment $\mathrm{Q}$ of the nucleus to the electric field gradient (efg) eq at the nucleus due to the surrounding electronic and nuclear charge distribution. ${ }^{(83,84)}$

The relevant interaction Hamiltonian is given in a laboratory-fixed frame $(L)$ as:

$$
\hat{H}_{\mathrm{Q}}(t)=\frac{\mathrm{e} Q}{2 I(I-1) \hbar} \sum_{m=-2}^{2}(-1)^{m} A_{\mathrm{m}}^{(2)} T_{-m}^{(2)}(t) \text {. }
$$

The $A_{m}^{(2)}$ are second rank irreducible tensor operators involving spin degrees of freedom only and the $T_{m}^{(2)}(t)$ are components of the second rank irreducible field gradient tensor. The latter are most conveniently expressed in a molecule fixed principal axis system $(P)$ where they become constants.

Conventionally, the efg tensor is characterized by the quadrupole coupling constant (QCC)

$$
\chi=h^{-1}(e Q)\left(e q_{z z}^{p}\right)
$$


and by the asymmetry parameter

$$
\eta=\left(q_{x x}^{\mathbf{p}}-q_{y y}^{\mathrm{p}}\right) / q_{z z}^{\mathrm{p}} \text {. }
$$

The transformation to the laboratory-fixed frame can be effected with the aid of the second rank Wigner rotation matrix. ${ }^{(121)}$ Incessant molecular jostling renders this transformation time-dependent. Hence quadrupole relaxation experiments provide a convenient tool for investigating orientational fluctuations in liquids.

Often molecular motions $\left(\tau_{\text {eff }}\right)$ are fast compared to currently available NMR frequencies $\left(\omega_{L}<10^{9} \mathrm{rad} \mathrm{s}^{-1}\right)$. The quadrupole relaxation then proceeds exponentially under extreme narrowing conditions at a frequency-independent rate given by:

$$
R_{1}^{\mathrm{Q}}=R_{2}^{Q}=\frac{3 \pi^{2}}{2} \frac{2 I+3}{I^{2}(2 I-1)}\left(\frac{\mathrm{e} Q}{h}\right)^{2} \int_{0}^{\infty}\left\langle T_{0}^{(2)}(\tau) T_{\mathrm{o}}^{(2)}(\mathrm{o})\right\rangle \mathrm{d} \tau .
$$

The integral over the electric field gradient time correlation function $G(\tau)$ defines an effective correlation time

$$
\tau_{\mathrm{Q}}(T, p)=\int_{0}^{\infty} G(\tau) / G(0) d \tau, \quad G(\tau)=\left\langle T_{\mathrm{o}}^{(2)}(\tau) T_{\mathrm{o}}^{(2)}(\mathrm{o})\right\rangle
$$

which provides a time scale for diffusive orientational fluctuations in liquids and depends on the state of the system $(T, p)$. In simple cases the correlation function $G(\tau)$ can be approximated by an exponential for times $t>\tau_{\mathrm{Q}}$.

$$
G(t)=\frac{3 \pi^{2}}{10} \mathrm{e}^{2}\langle q\rangle_{\mathrm{QLV}}^{2} \exp \left(-t / \tau_{\mathrm{Q}}\right)
$$

The sole effect of fast quasi-lattice vibrations (QLV) like hindered rotations (librations) is seen to average out part of the interaction ${ }^{(122,123,94)}$ leading to a librationally averaged QCC

$$
\langle\chi\rangle_{\mathrm{QLV}}=h^{-1} \mathrm{e}^{2} Q\langle q\rangle_{\mathrm{QLV}}=h^{-1} \mathrm{e}^{2} Q q_{z z}^{\mathrm{p}} \Gamma=\chi \Gamma .
$$

The motional averaging factor $\Gamma$ is given by:

$$
\Gamma^{2}=\sum_{m}\left|\left\langle D_{o, m}^{(2)}(\Omega)\right\rangle+\frac{\eta}{\sqrt{6}}\left[\left\langle D_{-2, m}^{(2)}(\Omega)\right\rangle+\left\langle D_{2, m}^{(2)}(\Omega)\right\rangle\right]\right|^{2}
$$

where $D_{m, m^{\prime}}^{(2)}(\Omega)$ are elements of the second rank Wigner rotation matrix and $\Omega$ denotes the Euler angles that transform from the molecule-fixed electric field gradient principal frame of nucleus $N$ to the librationally averaged molecular frame. The quadrupole relaxation of nuclei with spin $I=1$ is exceptional in that the Bloch equations hold even in the slow motions regime $\left(\omega_{L} \tau_{Q}>1\right)$. Spin-lattice $\left(R_{1}=1 / T_{1}\right)$ and spin-spin $\left(R_{2}=1 / T_{2}\right)$ relaxation rates are well-defined and may be expressed as:

$$
\begin{gathered}
R_{1}^{\mathrm{Q}}(I=1)=\frac{3 \pi^{2}}{20}\langle\chi\rangle^{2}\left[2 g_{2}\left(\omega_{\mathrm{L}} \tau_{\mathrm{Q}}\right)+8 g_{2}\left(2 \omega_{\mathrm{L}} \tau_{\mathrm{Q}}\right)\right] \\
R_{2}^{\mathrm{Q}}(I=1)=\frac{3 \pi^{2}}{20}\langle\chi\rangle^{2}\left[3 g_{2}(0)+5 g_{2}\left(\omega_{\mathrm{L}} \tau_{\mathrm{Q}}\right)+2 g_{2}\left(2 \omega_{\mathrm{L}} \tau_{\mathrm{Q}}\right)\right] .
\end{gathered}
$$

For nuclei with spin $I>1$ the quadrupole relaxation rate is non-exponential outside of the extreme narrowing regime. Hubbard ${ }^{(124)}$ has shown that the relaxation of both the longitudinal and the transverse components consist of $I$ decaying exponentials if $I$ is an integer and $(I+1 / 2)$ exponential terms if $I$ is half an odd integer. Explicit expressions were given for the case $I=3 / 2$. Halle and Wennerström ${ }^{(125)}$ reported a perturbation treatment of quadrupolar relaxation for $I=3 / 2,5 / 2,7 / 2$ and found a nearly exponential relaxation provided that the effective spectral density is only weakly frequency dependent. Transverse relaxation of these half-integer multipolar spins was discussed by Werbelow, ${ }^{(126)}$ Werbelow and Marshall ${ }^{(127)}$ and Westlund and Wennerström, ${ }^{(128)}$ who derived analytic expressions for the lineshapes under non-extreme narrowing conditions, including second order dynamic frequency shifts. 
3.2.4. Relaxation Through Chemical Shielding Anisotropy (CSA). An anisotropy in the chemical shielding tensor $\sigma$ may also contribute to nuclear relaxation. The relevant interaction Hamiltonian may be written as ${ }^{(103)}$

$$
\hat{H}_{\mathrm{CS}}=\gamma_{1} \sum_{L=0}^{2} \sum_{m=-L}^{L}(-1)^{m}\left(a_{1, m} A_{m}^{(L)} B_{0}\right) T_{-m}^{(L)}
$$

with the $A_{m}^{(\mathrm{L})}$ containing spin operators only, $B_{0}$ is the external magnetic field strength and the $T_{m}^{(\mathbf{L})}$ containing isotropic, symmetric and antisymmetric components of the chemical shielding tensor, which in general is not diagonal in a molecule-fixed axis system. ${ }^{(15)}$ The interaction becomes time dependent because of the rotational tumbling of the molecules and the relaxation of the magnetization components follow Bloch equations. In the simple case that the rotational tumbling of the molecules may be approximated by a Markov process characterized by a single correlation time $\tau_{\mathrm{L}}$ for a given $L$ the relaxation rates may be expressed as:

$$
\begin{aligned}
& R_{1}^{\mathrm{cs}}=\left(\eta_{1} B_{\mathrm{o}}\right)^{2}\left\{\frac{2}{3}\left(\sigma_{x y}^{2}+\sigma_{x z}^{2}+\sigma_{y z}^{2}\right) g_{1}\left(\omega_{\mathrm{L}} \tau_{1}\right)+\frac{2}{15} \Delta \sigma^{2}\left(1+\frac{\eta^{2}}{3}\right) g_{2}\left(\omega_{\mathrm{L}} \tau_{2}\right)\right\} \\
& R_{2}^{\mathrm{CS}}=\left(\gamma_{1} B_{\mathrm{o}}\right)^{2}\left\{\sigma_{\mathrm{lo}}^{2} \tau_{\mathrm{o}}+\frac{1}{3}\left(\sigma_{x \mathrm{y}}^{2}+\sigma_{x \mathrm{z}}^{2}+\sigma_{y z}^{2}\right) g_{1}\left(\omega_{\mathrm{L}} \tau_{1}\right)+\frac{1}{15} \Delta \sigma^{2}\left(1+\frac{\eta^{2}}{3}\right)\left[\frac{4}{3} \tau_{2}+g_{2}\left(\omega_{\mathrm{L}} \tau_{2}\right)\right]\right\} .
\end{aligned}
$$

The components of the chemical shielding tensor $\sigma$ are given in a molecule-fixed principal axis system of the symmetric part $\sigma^{(2)}$ of the tensor and

$$
\begin{gathered}
\sigma_{\mathrm{iso}}=(1 / 3)\left(\sigma_{x x}+\sigma_{y y}+\sigma_{z z}\right) \\
\Delta \sigma=\sigma_{z z}-\sigma_{\text {iso }} \\
\eta=\left(\sigma_{y y}-\sigma_{x x}\right) / \sigma_{z x} .
\end{gathered}
$$

It is seen that even under extreme narrowing conditions, $\omega_{L} \tau_{L} \ll 1$, the spin-lattice $\left(R_{1}\right)$ and spin-spin $\left(R_{2}\right)$ relaxation rates are unequal in contrast to the other relaxation mechanisms. One should note also the characteristic $B_{0}^{2}$ dependence of the relaxation rates which allows the CSA relaxation to be separated from other mechanisms.

3.2.5. The Spin-Rotation Relaxation. At high temperatures and low densities the reorientation of molecules in liquids proceeds rather freely. The rotating charge distribution generates local magnetic fields at the nuclei which fluctuate because of molecular collisions. ${ }^{(129)}$ The coupling of the magnetic moments of the nuclei to these fluctuating fields provides an efficient spin-rotation relaxation mechanism. The corresponding relaxation rate contains information about angular momentum correlation times $\tau_{\mathrm{J}}$. Theoretical expressions exist for asymmetric top molecules and anisotropic spin-rotation interactions for the anisotropic rotational diffusion model, ${ }^{(130)}$ the Fokker-Planck Langevin model ${ }^{(131)}$ and the extended diffusion model. ${ }^{(132)}$ However, these expressions are rather complex and have been used rarely to interpret experimental data. Often the simpler relaxation rate appropriate to a spherical top approximation is applied instead ${ }^{(133)}$

$$
R_{1}^{\mathrm{SR}}=\frac{8 \pi^{2}\langle I\rangle k T}{h^{2}}\left(C_{\mathrm{o}}^{2} \tau_{\mathrm{J}}+2 \Delta \mathrm{C}^{2} \tau_{\mathrm{SR}}\right)
$$

with the mean moment of inertia given by: ${ }^{(134)}$

$$
\langle I\rangle^{-1}=\frac{1}{3} \sum_{i=x, y, x} I_{i}^{-1}
$$

and the isotropic $C_{0}$ and anisotropic $\Delta C$ parts of the spin-rotation interaction tensor given by:

$$
C_{0}=\frac{1}{3} \sum_{i=x, y, z} C_{i i}, \Delta C=\frac{2}{3}\left[C_{z z}-\frac{1}{2}\left(C_{x x}+C_{y y}\right)\right]=C_{z z}-C_{0}
$$


The close similarity between the magnetic shielding and the spin-rotation coupling may be noted and was worked out quantitatively by Deverell; ${ }^{(135)} \tau_{\mathrm{J}}$ is the correlation time for the angular momentum correlation function, and $\tau_{S R}$ the correlation time of the angular velocity-orientational product correlation function. The latter may be expanded in terms of $\tau_{\mathrm{J}}$ and reduces to $\tau_{\mathrm{J}}$ in the diffusion limit where angular momentum and orientational correlation times are related by: ${ }^{(136)}$

$$
\tau_{1} \tau_{2}=\frac{\langle I\rangle}{c k T}
$$

with $c=6$ for a Brownian diffusion model and $c=2$ for a model involving reorientation by large angular jumps. ${ }^{(137)}$ Both correlation times depend, of course, strongly on the thermodynamic state of the system through the state variables $(T, p)$.

In liquids composed of almost spherical molecules a rough hard sphere approximation may become appropriate. Chandler ${ }^{(138)}$ has given a rigorous formulation of this model for the case of dense fluids $\left(\rho>2 \rho_{c}\right)$. The angular momentum correlation time $\tau_{\mathrm{J}}$ and hence the spin-rotation relaxation rate, should be proportional to the Enskog relaxation time $\tau_{E}$ of the hard sphere fluid:

$$
R_{1}^{\mathrm{SR}} \sim T_{\tau_{\mathrm{J}}}=\frac{T}{a(T)} \tau_{\mathbf{E}}(m, T, n, \sigma),
$$

with

$$
\frac{1}{\tau_{\mathrm{E}}}=\frac{8}{3}\left(\frac{\pi k T}{m}\right)^{1 / 2} \frac{n}{\sigma^{2}} g_{\mathrm{hs}}(\sigma) .
$$

The hard sphere contact pair distribution function may be evaluated quite accurately from the Carnahan-Starling approximation, ${ }^{(41)}$ eqn $(5)$. The roughness parameter $a(T)$ should be rigorously independent of density. It is given by: ${ }^{(139,140)}$

$$
a(T)=(\chi+1)^{-1}, \quad \chi=\frac{4\langle I\rangle}{m \sigma^{2}},
$$

in case of perfect rough hard spheres, and vanishes for perfectly smooth hard spheres.

3.2.6. Scalar Relaxation. The indirect spin-spin coupling $J$ between spins $I$ and $S$ may be modulated by two different mechanisms and hence can also cause relaxation. 'Scalar relaxation of the first kind' as introduced by Abragam $^{(83,141)}$ considers primarily the modulation of the indirect spin-spin coupling via the chemical exchange of nuclei (mostly protons) between different molecules. The relevant interaction Hamiltonian is given by: ${ }^{(\mathbf{8 3}, \mathbf{1 0 3})}$

$$
\hat{H}_{\mathrm{SC}}=\sum_{L=0}^{2} \sum_{m=-\mathrm{L}}^{\mathrm{L}}(-1)^{m} A_{m}^{(\mathrm{L})} T_{-m}^{(\mathrm{L})} .
$$

As usual the $A_{m}^{(\mathbf{L})}$ contain products of spin operators only and the $T_{m}^{(\mathbf{L})}$ in general contain isotropic, antisymmetric and symmetric components of the indirect spin-spin coupling tensor $J^{(142)}$ In addition, molecular tumbling also modulates the interaction because of possible anisotropic components of the indirect spin-spin coupling tensor $J$. A Bloch decay of the relaxation components results and the corresponding relaxation rates, assuming again a Brownian diffusion model for the tumbling motions, are given by: ${ }^{(103)}$

$$
\begin{aligned}
R_{1}^{S C}= & \frac{2}{9} S(S+1)\left\{3 J_{i s o}^{2} g\left(\omega_{i S}^{-} \tau_{0}\right)+\left(J_{x z}^{2}+J_{y z}^{2}+J_{x y}^{2}\right)\left[g\left(\omega_{1 S}^{-} \tau_{1}\right)+g\left(\omega_{1} \tau_{1}\right)\right]\right. \\
& \left.+\frac{1}{5} \Delta J^{2}\left(1+\frac{\eta_{1}^{2}}{3}\right)\left[\frac{1}{3} g\left(\omega_{1 S}^{-} \cdot \tau_{2}\right)+g\left(\omega_{1} \tau_{2}\right)+2 g\left(\omega_{1 S}^{+}+\tau_{2}\right)\right]\right\},
\end{aligned}
$$


with

$$
\begin{gathered}
\omega_{\mathrm{IS}}^{-}=\omega_{\mathrm{I}}-\omega_{\mathrm{S}} \\
\omega_{\mathrm{IS}}^{+}=\omega_{\mathrm{I}}+\omega_{\mathrm{s}} \\
\boldsymbol{R}_{2}^{\mathrm{sC}}=\frac{1}{9} S(S+1)\left\{3 J_{\mathrm{ise}}^{2}\left[\tau_{\mathrm{o}}+g\left(\omega_{\mathrm{IS}}^{-} \tau_{\mathrm{o}}\right)\right]+\left(J_{x y}^{2}+J_{x z}^{2}+J_{y z}^{2}\right)\left[g\left(\omega_{\mathrm{IS}}^{-} \tau_{1}\right)+g\left(\omega_{1} \tau_{1}\right)+2 g\left(\omega_{\mathrm{s}} \tau_{1}\right)\right]\right. \\
\left.+\frac{1}{5} \Delta J^{2}\left(1+\frac{\eta_{\mathrm{I}}^{2}}{3}\right)\left[\frac{4}{3} \tau_{2}+\frac{1}{3} g\left(\omega_{\mathrm{IS}}^{-} \tau_{2}\right)+2 g\left(\omega_{\mathrm{s}} \tau_{2}\right)+g\left(\omega_{\mathrm{I}} \tau_{2}\right)+2 g\left(\omega_{\mathrm{IS}}^{+} \tau_{2}\right)\right]\right\}
\end{gathered}
$$

with

$$
\begin{gathered}
J_{\mathrm{iso}}=\frac{1}{3} \sum_{i=x, y, z} J_{i i} \\
\Delta J=J_{z z}-J_{\mathrm{iso}} .
\end{gathered}
$$

In the case of vanishing anisotropic components of the $J$-coupling tensor the expressions simplify considerably with only the chemical exchange being effective. Also the non-extreme narrowing condition $\left(\omega \tau_{\mathrm{ex}} \gg 1\right)$ usually applies, hence $R_{2}^{\mathrm{SC}} \gg R_{1}^{\mathrm{SC}}$ and relaxation contributions from chemical exchange processes to spin-spin relaxation are much more pronounced. The correlation time $\tau_{\mathrm{o}}$ has to be identified with the exchange lifetime $\tau_{\text {ex }}$. Abragam ${ }^{(\mathbf{8 3 )}}$ also introduced the term 'scalar relaxation of the second kind' considering the possibility that the relaxation of nucleus $I$ results from the modulation of the indirect spin-spin coupling by the rapid (usually quadrupolar) relaxation of nucleus $S$. The scalar part of the interaction then gives rise to a relaxation contribution of the form:

$$
\begin{gathered}
R_{1}^{\mathrm{SC}}(I)=\frac{2}{3} S(S+1) J_{\text {iso }}^{2} g\left(\omega_{\mathrm{IS}}^{-} T_{2}(S)\right) \\
R_{2}^{\mathrm{SC}}(I)=\frac{1}{3} S(S+1) J_{\mathrm{iso}}^{2}\left[T_{1}(S)+g\left(\omega_{\mathrm{IS}}^{-} T_{2}(S)\right)\right],
\end{gathered}
$$

with $T_{1}(S)$ and $T_{2}(S)$ the corresponding relaxation times of nucleus $S$.

\subsection{Chemical Exchange}

Chemical exchange processes have a pronounced effect on the NMR lineshape as has been recognized since the early days of magnetic resonance. ${ }^{(143-147)}$ Information about changes in the environment of magnetic nuclei due to exchange between sites with different chemical shifts and/or different coupling constants can be derived from NMR spectra. The respective lineshapes, their chemical shift differences and amplitudes, give direct information about parts of the molecule affected by the molecular exchange, and even degenerate systems, in which the exchange leads to indistinguishable molecules, and also complicated multisite exchange processes, can be investigated by the dynamic NMR method. ${ }^{(148-151)}$ The dynamic information obtained in this way concerns primarily the rate constants and related thermodynamic parameters such as energies and volumes of activation for the processes studied. The phenomenological rate constants for the reaction are related to the reciprocal of the longest relaxation time for the reacting system. The rates are characterized by the strength of the coupling to the heat bath and it is the latter quantity which is strongly dependent on the density of the medium. The time scale of dynamic NMR experiments is such that rate constants in the range $10^{-1}-10^{6}(\mathrm{~Hz})$ can be measured by this method.

In the vast majority of dynamic NMR studies, the kinetic rate constants have been obtained by analysis of exchange-broadened spectra via lineshape analysis techniques. Various approximate as well as highly sophisticated lineshape analysis algorithms are available and are discussed thoroughly in several recent monographs. ${ }^{(85,152-154)}$

High pressure NMR has been used in this field mainly to study conformational transitions and dynamic solvent effects on exchange reaction rates in liquid solutions. Recent years have seen 
a renewed interest in reaction rate theory and the Kramers problem. ${ }^{(155-157)}$ Common chemical reactions may be classified conveniently as isomerization reactions, dissociation-recombination reactions and atom-transfer reactions. ${ }^{(15)}$ Isomerization reactions are unimolecular whereas atom-transfer reactions are bimolecular. Also, dissociation reactions are unimolecular, but recombination reactions are bimolecular. The ratio of the rate constants for the forward and backward reaction is related to the equilibrium constant by detailed balance. Classical theories for calculating rate constants can be divided into three groups: unimolecular rate theory of gases, ${ }^{(159)}$ transition state theory ${ }^{(160)}$ and the theory of diffusion-controlled reactions. ${ }^{(161)}$

Transition state theory is most widely used to interpret reaction rates $\Gamma_{\text {obs }}$ obtained from a NMR lineshape analysis. The resulting rate constant $\Gamma_{\text {TST }}$ depends on the properties of the reactant and on the solvent density through the potential of mean force. ${ }^{(162)}$ But transition state theory does neither explain the behaviour of unimolecular reactions at low pressures nor at high pressures. The theory of unimolecular reactions on the other hand predicts that at low pressures the rate constant increases linearly with solvent density due to energy activation. At high pressures, corresponding to high solvent densities and solvent viscosities, the rate constant becomes independent of density. In this regime, however, the crossing of the activation barrier is strongly hindered by frequent collisions with the solvent and the rate constant becomes proportional to the diffusion coefficient which in turn decreases with solvent density and viscosity.

According to modern theoretical models of reaction dynamics ${ }^{(163-167)}$ the reaction coordinate is coupled to the solvent through collisions between the solvent and solute molecules enabling the system to gain sufficient energy to cross the activation barrier. The observed rate constant $\Gamma_{\text {obs }}$ may be related to the transition state value through the use of a transmission coefficient $k$ which accounts for the collision effect on the reaction rate: ${ }^{(158)}$

$$
\Gamma_{\mathrm{obs}}=k(T, p) \Gamma_{\mathrm{TST}}=k(T, p) \Gamma_{\mathrm{o}} \exp \left(-\delta G^{*} / R T\right) ;
$$

$k$ is a complicated function of the collision frequency $1 / \tau_{v}$, and hence depends strongly on temperature and density.

The volume of activation for the reaction, obtainable from high pressure experiments, is defined as:

$$
\delta V^{*}=-(\mathrm{d} \ln \Gamma / \mathrm{d} p)_{\mathrm{T}}
$$

and may then be decomposed in a collisional contribution $\left(\delta V^{*}\right)$ coll and the usual transition state value $\left(\delta V^{*}\right)_{\text {TST }}$ according to:

$$
\begin{gathered}
\delta V^{*}=\left(\delta V^{*}\right)_{\mathrm{coll}}+\left(\delta V^{*}\right)_{\mathrm{TST}} \\
\left(\delta V^{*}\right)_{\mathrm{coll}} / k_{\mathrm{B}} T=-(\mathrm{d} \ln k / \mathrm{d} p)_{\mathrm{T}} \\
\left(\delta V^{*}\right)_{\mathrm{TST}} / k_{\mathrm{B}} T=-\left(\mathrm{d} \ln \Gamma_{\mathrm{TST}} / \mathrm{d} p\right)_{\mathrm{T}} .
\end{gathered}
$$

In the absence of electrostatic interactions the collision frequency can be related through simple hydrodynamics to the shear viscosity $\eta$ in an non-polar medium via:(168)

$$
1 / \tau_{\mathrm{v}}=\Phi / m=c(\pi \sigma / 2 m) \eta, c=\left\{\begin{array}{l}
6, \text { stick } \\
4, \text { slip }
\end{array}\right.
$$

with $\Phi$ the friction coefficient and $\sigma$ the hard-core diameter of the colliding particles.

The rate constant of an isomerization or dissociation-recombination reaction increases at low densities in proportion to the friction (solvent viscosity) or collision rate (density) due to energy activation. In a dense solvent it is proportional to the diffusion coefficient or inversely proportional to the friction coefficient $\left(D \approx k_{\mathrm{B}} T / \Phi\right)$ and decreases in the large friction regime (at high density). In between, the rate constant will reach a maximum value which is smaller than predicted by transition state theory. Hence by continuously increasing the density of the solvent from a dilute gas to a dense liquid, the system will pass through the energy activation regime to the diffusion-controlled regime. The relationship between reaction rates and solvent shear viscosity may break down, however, for high viscosity solvents at high packing fractions. ${ }^{(169-172)}$ 
High pressure experiments are useful in this respect as they allow the density and/or viscosity to be varied over a large range without changing the solvent. However, because of rapid intramolecular energy transfer the great majority of systems studied in dense liquid media exhibit high friction behaviour and the low friction, energy controlled regime and the Kramers turnover is only rarely observed in high pressure NMR experiments if at all.

Chemical exchange reactions can be studied also through their effect upon spin-lattice relaxation rates of nuclei involved in exchange processes. The relation between nuclear spin relaxation and chemical exchange is rather complicated. ${ }^{(87,88,173,174)}$ However, a simplified relationship is obtained if chemical exchange is slow compared to the molecular motions responsible for the relaxation. The exchange dynamics may then be taken into account by means of modified Bloch equations, ${ }^{(175)}$ by generalizing the equation of motion for the density operator ${ }^{(85)}$ or by using the theory of Markov processes. ${ }^{(176.177)}$ All exchange processes studied via lineshape analysis techniques belong to this category.

Early applications involved saturation transfer techniques ${ }^{(178-181)}$ which are suitable for measuring exchange rates in a regime where exchange lifetimes are of the same order of magnitude as the longitudinal relaxation times. The resulting rate constants are about an order of magnitude smaller than the smallest ones accessible by conventional lineshape analysis methods. Also relaxation in the rotating frame can be used to obtain exchange rates in about the same time window. ${ }^{(182)}$

Often exchange dynamics correspond to a situation where the nuclei studied exchange between different sites much faster than the rate of relaxation in each site but slow compared to molecular motions responsible for the relaxation. The observed spin-lattice relaxation rate is then given by:

$$
R_{1}=1 / T_{1}=\Sigma p_{i} R_{1 i}=\Sigma p_{i}\left(1 / T_{1 i}\right)
$$

where $p_{i}$ is the equilibrium fraction of the nuclei in site $i$ and $R_{1 i}\left(T_{1 i}\right)$ is the relaxation rate (time) in site $i$ in the absence of exchange. This approach presupposes an exponential relaxation at site $i$. The more complicated situation of non-exponential relaxation has also been treated..$^{(128,183)}$

A qualitatively different physical situation occurs when the mean residence time of a nucleus in a particular site $i$ is of similar magnitude or shorter than the relevant correlation times. Such a fast chemical exchange has the potential for shortening the correlation times in the expression for $R_{1}$. The influence of chemical exchange must then be included into the spin Hamiltonian modeling the spin-lattice interactions responsible for relaxation. ${ }^{(184,185)} \mathrm{A}$ rather general approach including even very fast intramolecular exchange processes together with a very lucid discussion has been given by Wennerström ${ }^{(173)}$ and has been extended recently to locally ordered fluids by Halle. ${ }^{(174,186,187)}$

\section{CHEMICAL EXCHANGE: EXPERIMENTS}

\subsection{Pressure Dependence of the Rate Constants of Chemical Reactions}

4.1.1. Ligand Exchange of Solvated Ions. Merbach and his group ${ }^{(6,12,13,188,189)}$ have made systematic studies of ligand exchange reactions of solvated metal ions in a wide variety of solvents. The appropriate NMR technique used in these investigations depends on the exchange rate. For very slow reactions, simple quenching experiments were used, where the extent of solvent exchange was determined by NMR after the sample has been exposed to high pressure at constant temperature for a defined period of time. For moderately fast reactions the influence of chemical exchange upon the spin-spin relaxation rate was employed through monitoring line widths. In the intermediate range the exchange was observed directly by conventional quantitative NMR spectroscopy at high pressure. The excellent and thorough reviews cited above collect all the original data published by this group. In this context it must suffice to describe the techniques applied and the mechanistic conclusions drawn by a few typical examples. The activation volume of the ligand exchange provides a unique and precise way of distinguishing between the various possible mechanisms of these reactions. In Fig. 7 the volume profile for the various possible reaction paths are given. A dissociative transition state (labelled D) must have a larger volume than the end products and thus be retarded by application of hydrostatic pressure. An activated complex formed by the associative inclusion (labelled A) of an 


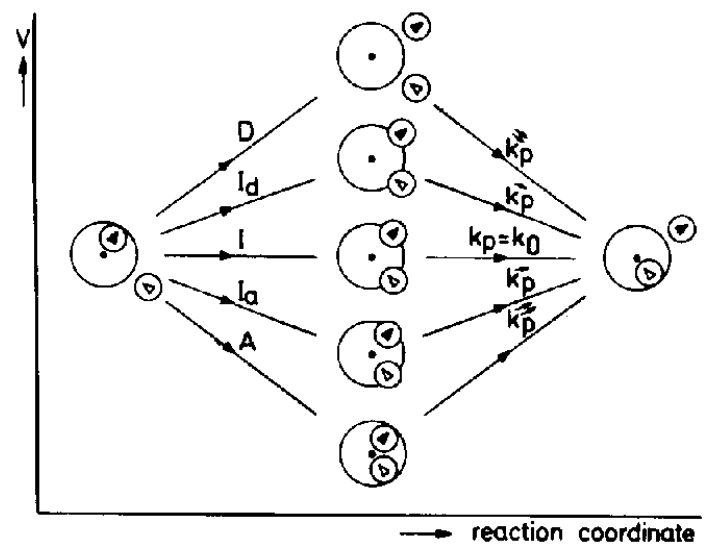

FIG. 7. Volume profile for ligand exchange reaction mechanisms. ${ }^{(6)}$

additional ligand into the first solvation shell should be more compact than the end products. It should thus be favoured by external pressure and the reaction should be characterized by a negative activation volume. Between these two extremes intermediate mechanisms (I) would be shown by a weaker pressure dependence.

In Table 1 a typical set of results ${ }^{(6)}$ is compiled, showing that within a series of trivalent diamagnetic compounds the mechanism may vary from one extreme to the other. This change is caused by differences in the diameter of the central ions and the volumes of the ligands. Figure 8 gives a typical example of the interesting pressure effects found in these systems. In Fig. 9 the temperature dependence of the linewidth at half height of the oxygen-17 signal of water enriched with this isotope is given for the complex $\left[\mathrm{Be}\left(\mathrm{H}_{2} \mathrm{O}\right)_{4}\right]^{2+}$ as a function of temperature. At higher temperatures the linewidth is determined by exchange of the water molecules between the complex and the bulk phase. $\left(\mathrm{Mn}\left(\mathrm{ClO}_{4}\right)_{2}\right.$ and $\mathrm{HClO}_{4}$ were added to the solution in order to suppress the signal of the bulk solvent and the hydrolysis effects of the bound water molecules.) The reciprocal linewidth passes through a shallow minimum at around $290 \mathrm{~K}$. At temperatures below this minimum the effective $T_{2}^{\mathrm{b}}$ is dominated by the increase of quadrupolar relaxation of the bound water molecules. The insert in Fig. 9 gives the

TABLE 1. Kinetic parameters for solvent exchange on diamagnetic trivalent cations in nitromethane as diluent ${ }^{(6)}$

\begin{tabular}{|c|c|c|c|c|c|c|c|}
\hline & $\begin{array}{l}r_{i}^{(\text {(a) }} \\
\text { pm }\end{array}$ & $\begin{array}{c}k_{1}^{298} \\
s^{-1}\end{array}$ & $\begin{array}{c}k_{2}^{298} \\
\mathrm{~s}^{-1} \mathrm{~mol}^{-1}\end{array}$ & $\underset{\mathrm{kJ} \mathrm{mol}^{-1}}{\Delta H_{+}^{+}}$ & $\begin{array}{c}\Delta S^{+} \\
\mathrm{JK}^{-1} \mathrm{~mol}^{-1}\end{array}$ & $\underset{\mathrm{cm}^{3} \mathrm{~mol}^{-1}}{\Delta V^{+}}$ & Mech. \\
\hline $\begin{array}{l}\mathrm{Al}\left(\mathrm{H}_{2} \mathrm{O}\right)_{6}^{3+(b)} \\
\mathrm{Al}(\mathrm{DMSO})_{6}^{3+(\mathrm{c})} \\
\mathrm{Al}(\mathrm{DMF})_{6}^{3+(\mathrm{c})} \\
\mathrm{Al}(\mathrm{TMPA})_{6}^{3+(\mathrm{c})}\end{array}$ & 54 & $\begin{array}{l}1.29 \\
0.30 \\
0.05 \\
0.78\end{array}$ & & $\begin{array}{l}84.7 \\
82.6 \\
88.3 \\
85.1\end{array}$ & $\begin{array}{l}+41.6 \\
+22.3 \\
+28.4 \\
+38.2\end{array}$ & $\begin{array}{r}+5.7 \\
+15.6 \\
+13.7 \\
+22.5\end{array}$ & $\begin{array}{l}\mathbf{I}_{d} \\
\mathrm{D} \\
\mathrm{D} \\
\mathrm{D}\end{array}$ \\
\hline $\begin{array}{l}\mathrm{Ga}\left(\mathrm{H}_{2} \mathrm{O}\right)_{6}^{3+(b)} \\
\mathrm{Ga}(\mathrm{DMSO})_{6}^{3+} \\
\mathrm{Ga}(\mathrm{DMF})_{6}^{3+} \\
\mathrm{Ga}(\mathrm{TMPA})_{6}^{3+}\end{array}$ & 62 & $\begin{array}{l}4.00 \\
1.87 \\
1.72 \\
6.4\end{array}$ & & $\begin{array}{l}67.1 \\
72.5 \\
85.1 \\
76.5\end{array}$ & $\begin{array}{r}+30.1 \\
+3.5 \\
+45.1 \\
+27.0\end{array}$ & $\begin{array}{r}+5.0 \\
+13.1 \\
+7.9 \\
+20.7\end{array}$ & $\begin{array}{l}\mathbf{I}_{d} \\
\mathrm{D} \\
\mathrm{D} \\
\mathrm{D}\end{array}$ \\
\hline $\begin{array}{l}\text { Sc(TMPA) })_{6}^{3+(b)} \\
\text { Sc(TMPA) })_{6}^{3+}\end{array}$ & 75 & 736 & $\begin{array}{l}85 \\
39\end{array}$ & $\begin{array}{l}34.1 \\
21.2\end{array}$ & $\begin{array}{r}-75.6 \\
-143.5\end{array}$ & $\begin{array}{l}-23.8 \\
-18.7\end{array}$ & $A, I_{n}$ \\
\hline $\operatorname{In}\left(\mathrm{TMPA}_{6}^{3+}\right.$ & 80 & & 7.6 & 32.8 & -188 & -21.4 & $A, I_{2}$ \\
\hline
\end{tabular}

(a) $r_{i}$ : ionic radii,

(b) In neat solvent,

(c) DMF: Dimethylformamide TMPA: Trimethylphosphate. 


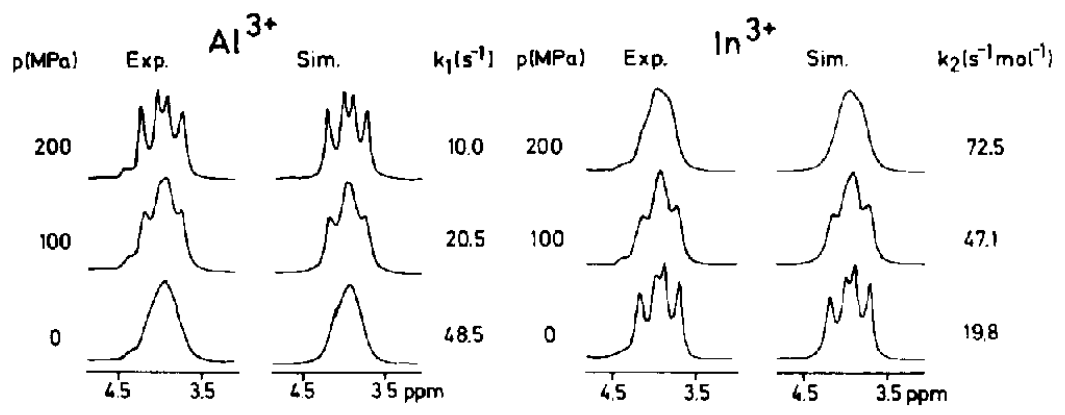

FIG. 8. Experimental and simulated $60 \mathrm{MHz}$ spectra of the protons in $\mathrm{M}(\mathrm{TMPA})\left(\mathrm{ClO}_{4}\right)_{3}$ dissolved in TMPA and $\mathrm{CD}_{3} \mathrm{NO}_{2}$ as function of pressure ${ }^{(6)}$ right: $\mathrm{M}=\mathrm{In}$. left: $\mathrm{M}=\mathrm{Al}$.

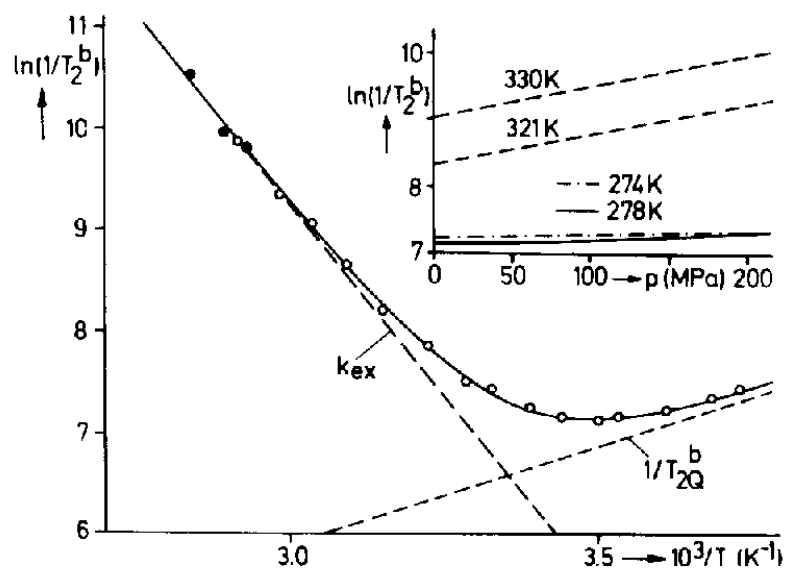

FIG.9. Temperature dependence of the relaxation rate, $1 / T_{2}^{b}$, of the liganded $-\mathrm{H}_{2} \mathrm{O} O-17$ signal of $0.1 \mathrm{~m}$ $\mathrm{Be}\left(\mathrm{H}_{2} \mathrm{O}\right)_{4}^{2+}$ with $\mathrm{Mn}\left(\mathrm{ClO}_{4}\right)_{2}$ and $\mathrm{HClO}_{4}$ added $(54.24 \mathrm{MHz})$. Insert: $p$-dependence of $1 / T_{2}^{\mathrm{b}}$ in the solutions given above. $330 \mathrm{~K}$ and $321 \mathrm{~K}$ isotherms=exchange region. $274 \mathrm{~K}$ and $278 \mathrm{~K}$ isotherms=quadrupolar region $(27.11 \mathrm{MHz})^{(12)}$

pressure dependence of the two relaxation rates. The associative exchange of water molecules shows an activation volume of $-13.6 \mathrm{~cm}^{3} \mathrm{~mol}^{-1}$ and is enhanced by external pressure, while the diffusive tumbling of water molecules and the tetraaquo complex remains almost uninfluenced. In Fig. 10 the pressure dependence of the exchange of water molecules in the octahedral hexaquo complexes of

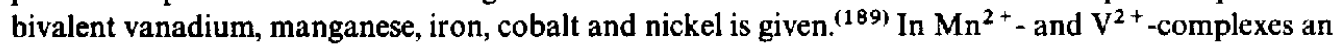
acceleration of the exchange rate with pressure is found, indicative of an associative mechanism, while for $\mathrm{Ni}^{2+}, \mathrm{Co}^{2+}$ and $\mathrm{Fe}^{2+}$ a dissociative exchange mechanism is shown to prevail. These qualitative differences observed in a series of cations with rather similar diameters can be tentatively explained by the influence of ligand field effects, stemming from the d-electron configurations of the various ions, upon the exchange mechanism. A similar study has recently been published by Mizuno et al. ${ }^{(190)}$

4.1.2. Intramolecular Conformational Transitions. The analysis of exchange broadened high resolution spectra offer a unique possibility for determining the activation parameter for intramolecular

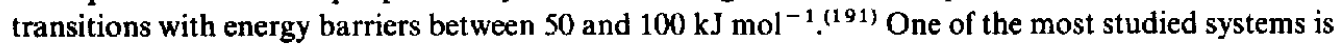
the rotation of the amide group.

Simple preparative methods yield a large variety of products and thus permit systematic studies of the relative influence of sterical and quantum mechanical effects upon the partial double bond 


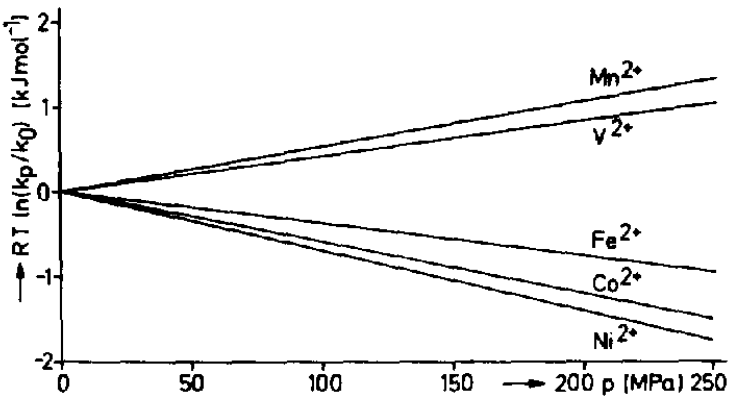

FiG. 10. Effect of pressure on the water exchange rates for divalent ions. ${ }^{(12)}$

character of the carbonyl $\mathrm{C}-\mathrm{N}$ bond. These compounds have been very well studied at ambient pressure and the data collected have been used for a comprehensive quantitative characterization of this dynamic process. ${ }^{(192)}$ In the late 70 s our group started to extend these data to high pressures in order to learn whether the density/pressure effects observable would aid in a general description of the density dependence of the rates of intramolecular rearrangements. In Table 2 the systems analyzed are compiled. Figure 11 gives the pressure effects upon the methyl proton spectra of $N, N$-diisopropylbenzamide together with the rates obtained from the simulation of these spectra. An activation volume of $+5.4\left(10^{-3} \mathrm{~m}^{3} \mathrm{~mol}^{-1}\right)$ is obtained from the rate constants.

In Table 3 the influence of the solvent upon the activation volumes of three $N, N$-dimethylamides is given. The increase of $\Delta G^{*}$ with solvent polarity is generally explained by a preferential stabilisation of the more polar planar ground state by the solvent molecules through hydrogen bonding or dipolar interactions. Attempts to correlate the trend found in the free activation enthalpies with the observed volume $\Delta V^{*}=8 \pm 2\left(10^{-3} \mathrm{~m}^{3} \mathrm{~mol}^{-1}\right)$ effects failed. $\Delta V^{*}$ does not vary systematically with solvent polarity. It is worth mentioning an exception in the aqueous solution case, where a very unexpected concentration dependence of $\Delta V^{\#}$ is found. ${ }^{(204)}$ At constant temperature, the inversion rate of all amides studied here decreases linearly as a $\log k$ versus $p$ plot, within the precision of the data. (The pressure range studied was between 0.1 and $150 \mathrm{MPa}$ in most cases, and in a few examples $p$ was raised to $200 \mathrm{MPa}$.) The results obtained in the non-aqueous solvents for all amides and dimethylamides studied can be well explained by the simple sterical consideration given in Fig. 12 .

TABLE 2. Pressure dependence of conformational dynamics studied through the analysis of exchange broadened high resolution ${ }^{1} \mathrm{H}$ NMR spectra

Amides with two identical N-substituents:

Formamide, Acetamide:

$N, N$-Dimethylformamide, -acetamide and -benzamide

Benzamides with larger $N$-substituents

$(193,194)$

$(195,196)$

(194)

(197)

$(198,199)$

Various $N, N$-disubstituted amides of 1 naphthoic-and 9-anthracencarboxylic acid

$N$-methyl- $N$-isopropyl acetamide

Glycylsarcosine, $\mathrm{N}$-acetyl-2-proline- $\mathrm{NH}$-methylamide

Cyclohexane

(202)

substuted $N$-aralkylpyridinium

Lumazine- $\beta$-Riboside

$(204,196)$ 


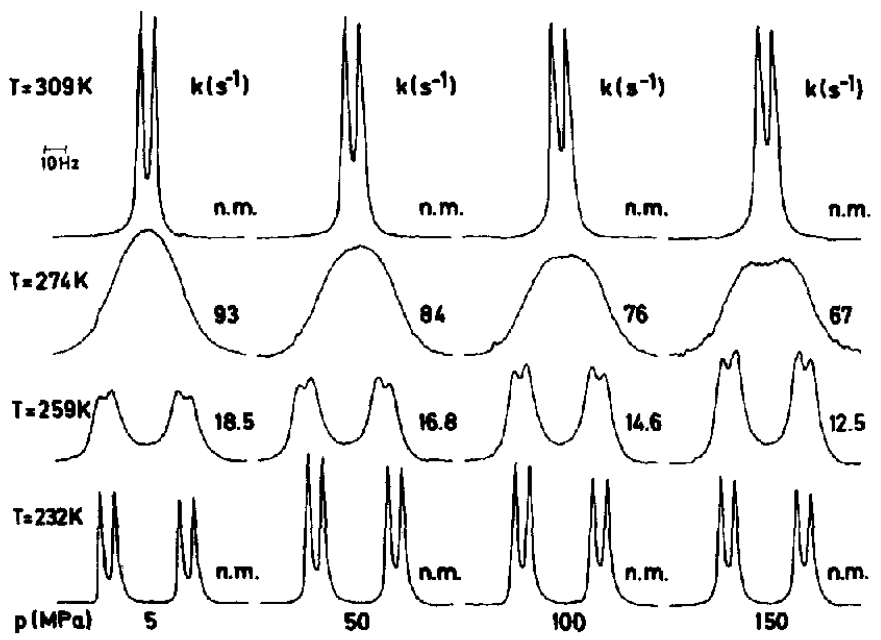

FIG. 11. Influence of pressure upon the exchange broadened signals from the protons in the methyl groups on $N, N$-diisopropyl-benzamide ${ }^{(198)}(100 \mathrm{MHz}) . k\left(\mathrm{~s}^{-1}\right)$ : exchange rates obtained from simulation. (n.m. $=$ not measured).

TABLE 3. Free activation enthalpies and activation volumes derived for the various solutions of $N, N$-dimethylformamide (DMF), $N, N$-dimethylacetamide (DMA) and $N, N$-dimethylbenzamide (DMB). Concentrations are vol $\%$ at $25^{\circ} \mathrm{C}$ and 1 bar

\begin{tabular}{|c|c|c|c|c|c|c|c|c|c|}
\hline \multirow[b]{2}{*}{ Solvent } & \multicolumn{3}{|c|}{ DMF } & \multicolumn{3}{|c|}{ DMA } & \multicolumn{3}{|c|}{ DMB } \\
\hline & $\underset{\mathrm{kJ}}{\Delta G^{*}}$ & $\begin{array}{c}\text { (5 MPa) } \\
\text { lit }\end{array}$ & $\begin{array}{l}\Delta V^{*} \\
\mathrm{~cm}^{3} \\
\mathrm{~mol}\end{array}$ & $\underset{\mathrm{kJ}}{\Delta G^{*}}$ & $\begin{array}{c}(5 \mathrm{MPa}) \\
\text { lit }\end{array}$ & $\begin{array}{l}\Delta V^{*} \\
\mathrm{~cm}^{3} \\
\mathrm{~mol}\end{array}$ & $\underset{\mathrm{kJ}}{\Delta G^{*}}$ & $\begin{array}{c}\text { (5 MPa) } \\
\text { lit }\end{array}$ & $\begin{array}{l}\Delta V^{\prime} \\
\mathrm{cm}^{3} \\
\mathrm{~mol}\end{array}$ \\
\hline Neat & & $88^{(1)}$ & & 76 & $76^{(1)}$ & 7.6 & & & \\
\hline $\begin{array}{l}60 \% \mathrm{CCl}_{4} \\
20 \% \mathrm{HMDSiO}^{2}\end{array}$ & 86.5 & $88^{(1)}$ & 7.8 & 73.5 & $72^{(3)}$ & 5.5 & 61.5 & & 9.6 \\
\hline $80 \% \mathrm{C}_{6} \mathrm{D}_{6}$ & 87 & & 9.8 & 73.5 & & 9.0 & 61.5 & $61.5^{(4)}$ & $8.1^{\prime \prime}$ \\
\hline $\begin{array}{l}60 \%\left(\mathrm{CD}_{3}\right)_{2} \mathrm{CO} \\
20 \% \text { TMS }\end{array}$ & 87 & $88^{(2)}$ & 9.4 & 75 & $75^{(1)}$ & 10.0 & 62.5 & $62^{(4)}$ & 8.6 \\
\hline $80 \%\left(\mathrm{CD}_{3}\right)_{2} \mathrm{SO}$ & & & & 77 & $78^{(1)}$ & 6.8 & & & \\
\hline $80 \% \mathrm{CD}_{3} \mathrm{CN}$ & 87.5 & & 9.1 & 76 & & 9.3 & 65 & $65^{(5)}$ & 9.5 \\
\hline $80 \% \mathrm{CD}_{3} \mathrm{OD}$ & 90.5 & & 9.6 & 79.5 & & 7.6 & 67.5 & & 12.9 \\
\hline $80 \% \mathrm{D}_{2} \mathrm{O}$ & 92 & & 6.0 & 81 & $81^{(1)}$ & 1.6 & 72.5 & $76^{(b)(4)}$ & 8.9 \\
\hline
\end{tabular}

\footnotetext{
(a) $40 \% \mathrm{C}_{6} \mathrm{D}_{6} / 40 \% \mathrm{CD}_{3} \mathrm{C}_{6} \mathrm{D}_{3}$.

(b) $93 \% \mathrm{D}_{2} \mathrm{O}$.

(1) Drakenberg et al. (205)

(2) Whittaker and Siegel(206).

(3) Reeves et al. (207).

(4) Jackman et al.(208)

(5) Spaargaren et al.(209).
}

The description implies that during the $180^{\circ}$-flips the complete rotational toroid has to be kept free of solvent molecules. This is obviously reasonable only as long as the rotational motion of the amide group is fast compared with the translational diffusive mobility of the surrounding molecules. Crude estimates ${ }^{(198)}$ show that this condition might be fulfilled for the amides and the dimethylamides but certainly not for the larger dialkyl- and aryl-substituents. 

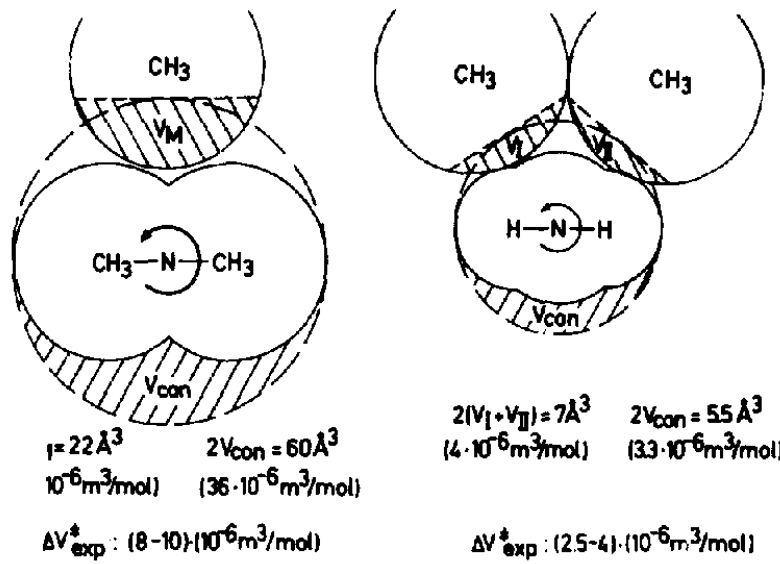

$$
\begin{gathered}
\left.2 \mid V_{1}+V_{T}\right)=7 A^{3} \quad 2 V_{\text {con }}=55 A^{3} \\
\left(4 \cdot 10^{6} \mathrm{~m}^{3} \mathrm{~mol}\right) \quad\left(33 \cdot 10^{6} \mathrm{~m}^{3} / \mathrm{mol}\right) \\
\left.\Delta V_{\text {axp }}^{*}:(2.5-4) \cdot 110^{-6} \mathrm{~m}^{3} / \mathrm{mol}\right)
\end{gathered}
$$

Fig. 12. Van der Waals radii of the amide and dimethyl-amide group in a solvent of methyl groups. $V_{M}$ resp. $V_{\mathrm{I}}=V_{\mathrm{II}}$ indicate the maximal volume that can be occupied by the methyl groups.

With the exception of the aqueous solutions of the dimethylamides, the concentration and solvent dependence of $\Delta V^{*}$ is rather regular. In the dilute aqueous solutions however a very steep decrease of $\Delta V^{*}$ is observed, while the free activation enthalpy is unaffected. ${ }^{(204)}$ In Fig. 13 the data for dimethylacetamide in some solvents are given. This unique drop in $\Delta V^{\text {* }}$ with dilution occurs in all dimethyl amides but is shifted to lower concentrations with increasing size of the hydrophobic carbonyl-substituent. It may be explained by the formation of an open hydrate shell around the apolar $\mathrm{N}-\left(\mathrm{CH}_{3}\right)_{2}$ moiety, that can only develop at sufficiently low concentrations, where associates, formed by hydrophobic interaction can no longer interfere. Figure 14 shows $\Delta V^{*}$ (c) for the higher amides studied. For the cyclohexane ring inversion we have observed ${ }^{(195)}$ for the first time an acceleration with pressure, characterized by an activation volume of $\Delta V^{*}=(-1.9 \pm 0.5) \times 10^{-3} \mathrm{~m}^{3} \mathrm{~mol}^{-1}$. For this process, as for all other conformational transitions studied by our group, a pressure independent activation volume was found within the precision of the data in the pressure range studied. Some of the data collected by Jonas and his group ${ }^{(210)}$ were obtained at considerably higher pressures, (up to $500 \mathrm{MPa}$ ), and at higher magnetic fields. These experiments proved for the first time that the

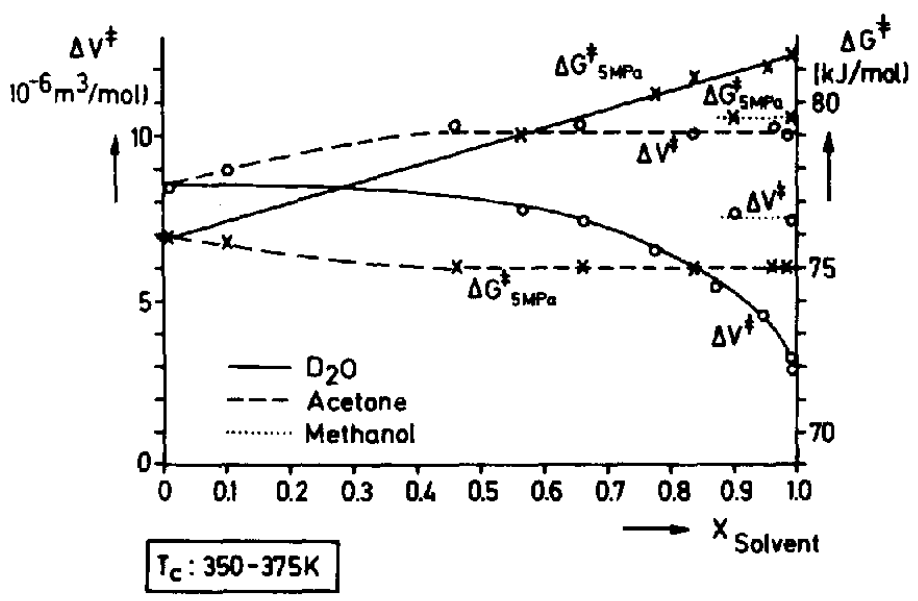

FIG. 13. Concentration dependence of the free enthalpy of activation and the activation volume for the rotation of the dimethylamide group in dimethylacetamide in various solvents. ${ }^{\text {(204) }}$ 


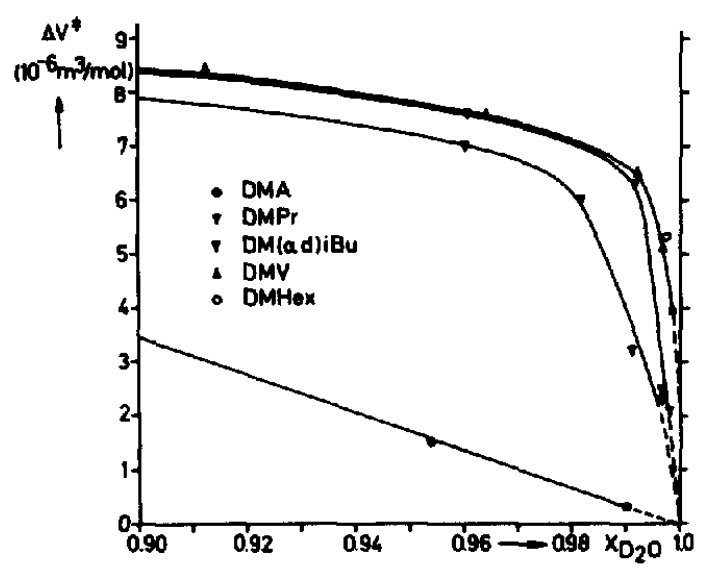

Fig. 14. Concentration dependence of the activation volumes of a series of dimethylamides in aqueous solution. (204) DMA: dimethylacetamide, DMPr: dimethylpropioamide, DMiBu: dimethylisobutylamide, DMV: dimethylvalinamide, DMHex: dimethylhexamide.

transmission coefficient $k$ for the cyclohexane- and 1,1-difluorocyclohexane ring inversion ${ }^{(211-215)}$ is indeed pressure dependent.

In Fig. 15 the normalized inversion rates as a function of solvent viscosity are given. The lower isomerization rates were determined by classical NMR line shape analysis ${ }^{(212)}$ whilst the higher ones were obtained from the analysis of proton rotating frame relaxation times. ${ }^{(215)}$ In Fig. 16 the normalized transmission coefficients for the cyclohexane ring inversion in $\mathrm{CS}_{2}$ as a function of viscosity is given for two temperatures. From these experiments it can be safely concluded that the barrier height of the isomerization is truly independent of pressure in the range studied and the isomerization of cyclohexane is in the inertial regime of the Kramers model.

The rotation of the coordinated ethylene in the complex $\mathrm{C}_{5}-\mathrm{H}_{5} \mathrm{Rh}\left(\mathrm{C}_{2} \mathrm{H}_{4}\right)\left(\mathrm{C}_{2} \mathrm{~F}_{4}\right)$ is initially enhanced by pressure, passes through a maximum around $300 \mathrm{MPa}$ and decreases at higher pressures.

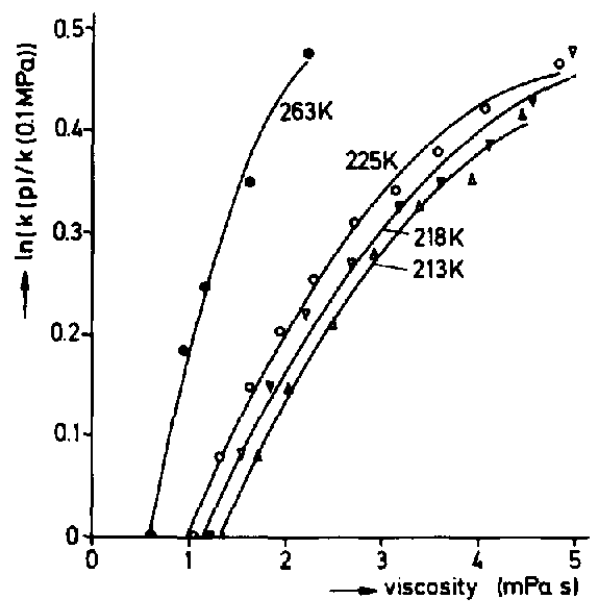

FIG. 15. Normalized isomerization rates as function of viscosity for the cyclohexane ring inversion in $\mathrm{CS}_{2}$. Closed symbols: NMR rotating frame relaxation. Open symbols: line shape analysis. ${ }^{(214.215)}$ 


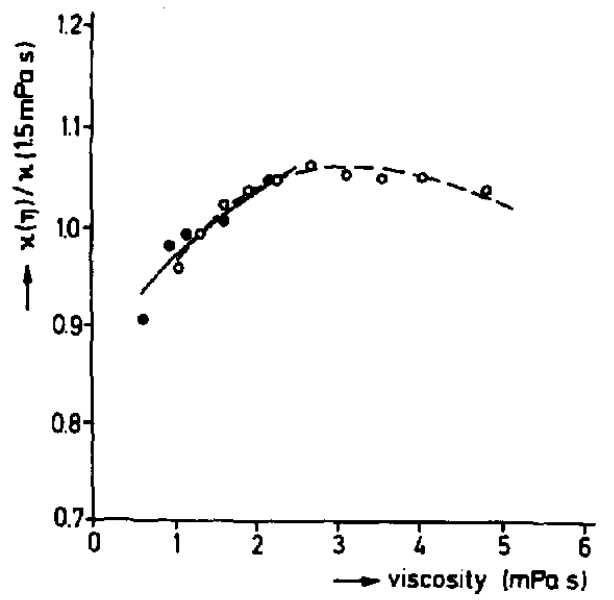

FIG. 16. Normalized transmission coefficient as a function of viscosity for the cyclohexane isomerization (data from Fig. 15).
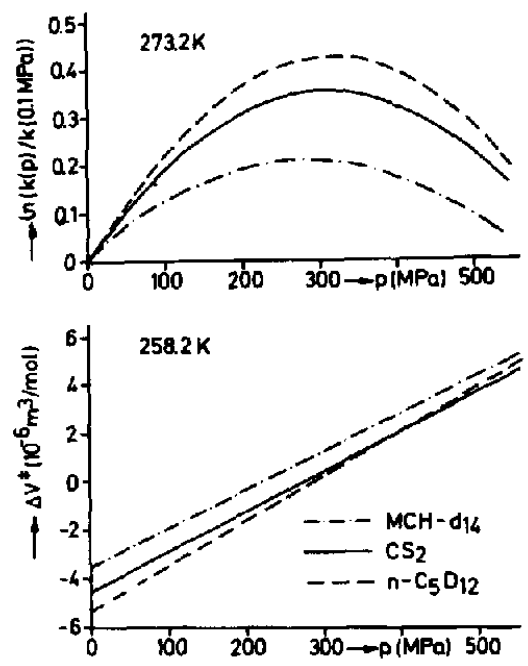

Fig. 17. Top: Normalized rotation rate as a function of pressure or the ethylene group in $\mathrm{C}_{5} \mathrm{H}_{3} \mathrm{Rh}\left(\mathrm{C}_{2} \mathrm{H}_{4}\right)\left(\mathrm{C}_{2} \mathrm{~F}_{4}\right)$. Bottom: Experimental activation volume as a function of pressure Methylcyclohexane-d $14\left(\mathrm{MCH}-\mathrm{d}_{14}\right)-\mathrm{CS}_{2}-$ $n$-pentane $d_{12}{ }^{(210)}$

In Fig. 17 the normalized rates for this motion are given. The pressure dependence of $\Delta V_{\mathrm{obs}}^{*}$ is derived from the rates. The Kramers turnover for this transition occurs at the relatively high viscosity of $\approx 7$ (mPas) in methylcyclohexane while for $\mathrm{CS}_{2}$ and $n$-pentane- $\mathrm{d}_{12}$ the turnover point is $\approx 2$ (mPas). Wagner ${ }^{(216)}$ applied the analysis of the pressure dependence of exchange broadened spectra to the small protein basic pancreatic trypsin inhibitor. He could determine flip rates of the aromatic rings of a phenylalanine and a tyrosine and derived activation volumes of $\sim 30\left(10^{-3} \mathrm{~m}^{3} \mathrm{~mol}^{-1}\right)$ for these transitions. $\Delta V^{*}$ was independent of pressure in the range studied $(0.1-120 \mathrm{MPa})$. 


\section{SELF-DIFFUSION COEFFICIENTS: EXPERIMENTS}

\subsection{Xenon}

Xenon is the only monoatomic liquid in which the self-diffusion coefficient has been studied over a wide $p, T$-range. ${ }^{(217)}$ The data have been obtained between $273 \mathrm{~K}$ and $343 \mathrm{~K}$ up to a maximum pressure of $135 \mathrm{MPa}$. The experimental isotherms for xenon are given in Fig. 18. The authors state that these results are well described at intermediate and high densities by the hard spheres MD simulations with a hard sphere diameter $r=0.382 \mathrm{~nm}$. However, the agreement with a simulation of a Lennard-Jones liquid is only qualitative. The deviations from the analytic Enskog model are large. A thorough test of the rough hard spheres model (RHS) and the interacting spheres model can be achieved only after data have been collected over a wider $\rho, T$-range.

\subsection{Lower Alkanes}

Methane at fluid densities has been studied over the widest $p, T$-range. The data collected by two groups, ${ }^{(218-220)}$ extend from $110 \mathrm{~K}$ to $450 \mathrm{~K}$ up to maximum pressures of $200 \mathrm{MPa}$. In the region of overlap the agreement between the two data sets is within the accuracy stated. The non-polar, conformationally rigid and almost spherical molecule provides the most stringent test for the models discussed in the theory section. Methane is quantitatively characterized by the RHS model. Fitting the individual isotherms of $D$ to eqn (9), yields an $A$-parameter between 1.07 and 0.94 ; no trend for the temperature dependence of $A$ is apparent. Methane is also quantitatively described by the IS-model. In Fig. 19 the reduced self diffusion coefficients $D^{*}=D_{\text {exp }} / D_{0}\left(r_{B}\right) n$ for methane are compiled. The fit is best, if the exponential term in eqn (11) is omitted, and only the temperature dependence of $r_{B}$ as given in eqn (10) is considered. In Table 4 the fit parameters obtained are given.

For methane, the explicit inclusion of the attractive interaction via the exponential term reduces the quality of the fit, while for all other substances studied hitherto the standard deviation of the fit is significantly reduced. The only exception to this is tetrafluoromethane ${ }^{(221)}$ which is also included in Table 4. Among approximately twenty compounds studied only the two rigid, symmetric and non-polar substances $\mathrm{CH}_{4}$ and $\mathrm{CF}_{4}$, show this behaviour, a finding not considered to be fortuitous.

In Fig. 19 the $D^{*}$ values for propane are also given. The deviation from the 'theoretical' curve is representative of the behaviour of most other substances. The fitting of the data for propane becomes perfect, if the exponential term is included. The result is given in Fig. 20.

In recent years molecular dynamics simulations of the lower alkane liquids have advanced to a state where thermodynamic properties can be extracted with reasonable accuracy..$^{(222-224)}$ The static

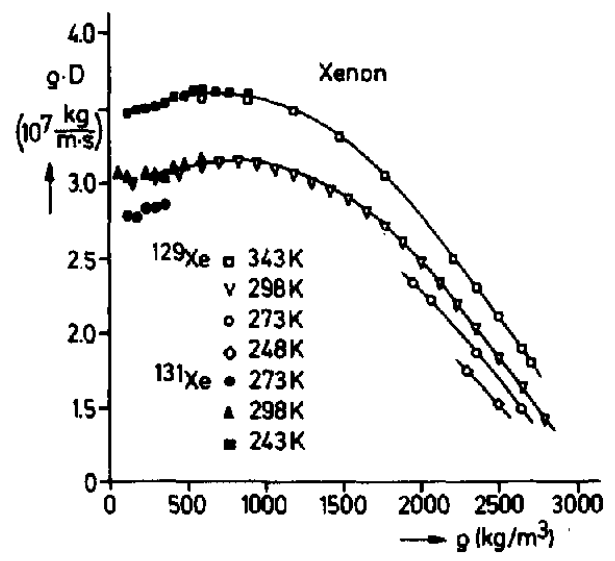

FiG. 18. Isotherms of the self-diffusion coefficients $D$ in liquid xenon as function of the density $\rho .^{(217)}$ 


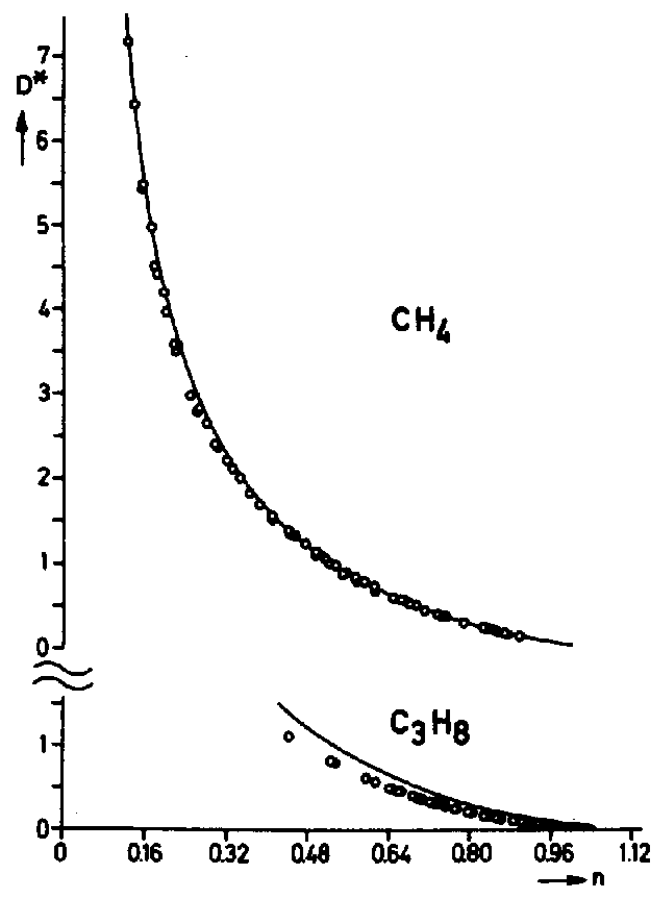

Fig. 19. Reduced self-diffusion coefficients $D^{*}=D_{\operatorname{mip}} / D_{0}\left(\sigma_{\mathrm{B}}\right) n$ for methane and propane as function of the packing density $n=\rho \sigma^{3}$. The solid lines result from a fit of the experimental data to eqn (11) under omission of the exponential term.

TABLE 4. Lennard-Jones parameter obtained from fitting the self-diffusion data to eqn (11). $A$ : without the exponential term $B$ : including the exponential term

\begin{tabular}{|c|c|c|c|c|c|c|c|c|}
\hline \multirow[b]{2}{*}{ Substance } & \multirow[b]{2}{*}{$\begin{array}{c}T \text { range } \\
(\mathrm{K})\end{array}$} & \multirow[b]{2}{*}{$n$} & \multicolumn{3}{|c|}{$A$} & \multicolumn{3}{|c|}{$\boldsymbol{B}$} \\
\hline & & & $\underset{(\mathrm{nm})}{\sigma_{\mathrm{L}}}$ & $\begin{array}{l}\varepsilon / k_{\mathrm{B}} \\
(\mathbf{K})\end{array}$ & $\begin{array}{c}\text { Std } V . \\
(\%)\end{array}$ & $\underset{(\mathrm{nm})}{\sigma_{\mathrm{LJ}}}$ & $\begin{array}{l}\varepsilon / k_{\mathrm{B}} \\
(\mathrm{K})\end{array}$ & $\underset{(\%)}{\operatorname{Std} V .}$ \\
\hline $\begin{array}{l}\mathrm{CH}_{4} \\
\mathrm{CF}_{4} \\
\mathrm{C}_{2} \mathrm{H}_{6} \\
\mathrm{C}_{3} \mathrm{H}_{8}\end{array}$ & $\begin{array}{l}110-454 \\
140-440 \\
136-454 \\
112-453\end{array}$ & $\begin{array}{l}0.12-0.89 \\
0.2-0.98 \\
0.28-1.00 \\
0.44-1.04\end{array}$ & $\begin{array}{l}0.417 \\
0.440 \\
0.450 \\
0.479\end{array}$ & $\begin{array}{l}26.7 \\
244 \\
117.8 \\
575.0\end{array}$ & $\begin{array}{r}2.3 \\
6.3 \\
4.1 \\
17.6\end{array}$ & $\begin{array}{l}0.422 \\
0.452 \\
0.456 \\
0.483\end{array}$ & $\begin{array}{c}19.8 \\
88 \\
70.8 \\
210.9\end{array}$ & $\begin{array}{l}2.6 \\
6.8 \\
2.0 \\
2.8\end{array}$ \\
\hline
\end{tabular}

properties are well described by skeleton models, where the individual methyl and methylene groups are each represented by a single Lennard-Jones centre.

The merit of the different approximations used could be critically tested by a comparison of dynamic quantities derived from the simulations with experimental results. The most basic dynamic property, the single-particle self-diffusion coefficient, would be the appropriate choice. In Fig. 21 our experimental self-diffusion data for propane are compared with the simulation results of Toxvaerd. ${ }^{(224)}$ The simulated data were obtained for three-site skeleton models with different bond lengths, bond angles, diameters and well depths of the Lennard-Jones potentials. Comparison with our experimental results, that are quantitatively represented by a single spherical Lennard-Jones potential, leads to the suggestion that at least the dynamic properties of the conformationally rigid lower alkanes can be simulated by an even simpler molecular model. The comparison also shows that $D$ is not very sensitive to the molecular geometry and the potential chosen. 


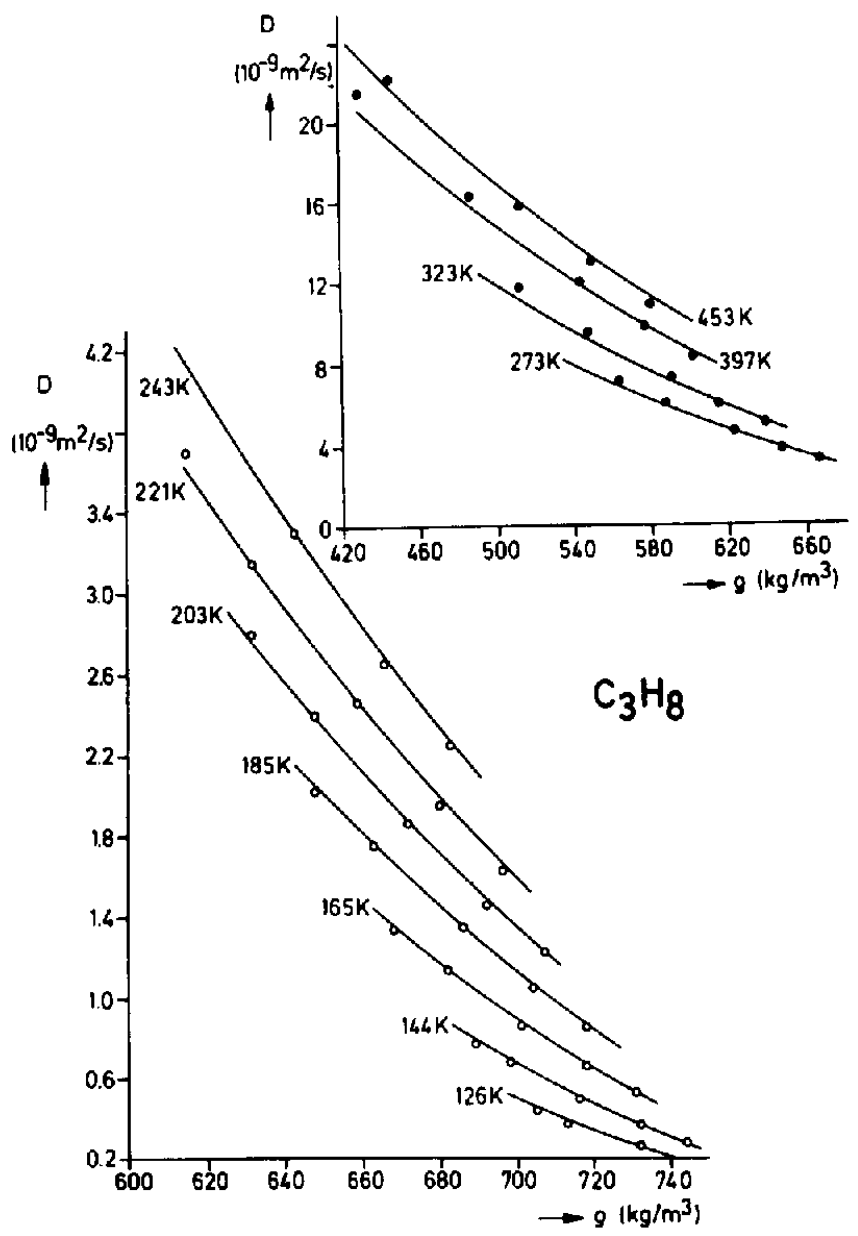

FIG. 20. Fit of the self-diffusion coefficient $D$ in fluid propane by the interacting spheres model eqn (11) as function of the density $\rho$.

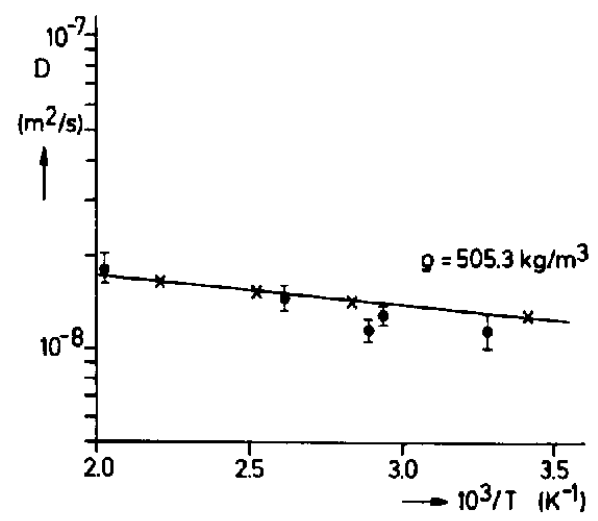

FIG. 21. Comparison of the experimental and calculated self-diffusion coefficients in fluid propane. Results of simulation $^{(22)}$ interpolated experimental points ${ }^{(220)}$ at the constant density $\rho=505.3 \mathrm{~kg} \mathrm{~m}^{-3}$. 


\subsection{Halomethanes}

Tables 5 and 6 show the results of the analysis of the self-diffusion data for several halomethanes. Although $D$ is described well by the rough hard sphere model, an unconstrained fit of the individual isotherms yields a rotation translation coupling parameter $A$ that decreases with falling temperature, which is not in accord with the purely geometrical description of $A$ as offered by Chandler. ${ }^{(44,138,140)}$ These experimental results led Speedy et $a l^{(45)}$ to analyze the data with the interacting sphere model, considering attractive interactions between the molecules by a Lennard-Jones potential. The relatively large standard deviations given for the fitting process could result from the approximate nature of the description of symmetric top or asymmetric molecules by a single Lennard-Jones potential. However, for the time being this analysis is at the mercy of relatively poor density data, obtained in many cases by crude and wide extrapolations of experimental results. At high packing fractions, a $2 \%$ error in the density will result in $\sim 20 \%$ deviations for the self diffusion coefficients.

The value of this type of analysis could be tested thoroughly by the study of $D$ and $\rho$, the packing fraction, over a very wide pressure and temperature range. De Zwaan et al..$^{(232)}$ and Harris et al. (231) succeeded in describing self diffusion in $\mathrm{CFCl}_{3}$ and $\mathrm{CHCl}_{3}$ by the rough hard sphere model (Table 6) and obtaining reasonable standard deviations. However, their data do not extend over a sufficiently large temperature range. The diameters derived by both fitting procedures agree well with the geometric dimensions as obtained from crystal data. The well depth $\varepsilon$ of the Lennard-Jones potential neither correlates with the dipole moment of the molecules in the series of the fluoro- and chloromethanes, nor does it follow the changes in the polarizability estimated from the atomic contributions. A similar statement applies for the $A$ parameter. Neither the temperature dependence nor the absolute values appear to correlate quantitatively with the deviation of the molecules from spherical symmetry, or with the dipole moments and polarizability differences. It is obvious that the description of these compounds by spherically symmetric potentials and a spherical geometry averages over the properties

TABLE 5. Fit of diffusion data to the interacting sphere model

\begin{tabular}{|c|c|c|c|c|c|c|}
\hline Substance & Reference & $T / \mathbf{K}$ & $\rho \sigma^{3}$ & $\sigma_{\mathrm{LJ}} / \mathrm{nm}$ & $\left(\varepsilon / k_{\mathrm{B}}\right) / \mathrm{K}$ & $\%$ Std. dev. \\
\hline $\mathrm{CH}_{3} \mathrm{Cl}$ & Prielmeier et al..$^{(225)}$ & $210-440$ & $0.53-0.88$ & 0.436 & 129 & 8.2 \\
\hline $\mathrm{CH}_{2} \mathrm{Cl}_{2}$ & Prielmeier et al..$^{(225)}$ & $186-406$ & $0.65-1.02$ & 0.479 & 162 & 3.3 \\
\hline $\mathrm{CHCl}_{3}$ & Prielmeier et al. ${ }^{(225)}$ & $233-397$ & $0.73-0.98$ & 0.534 & 107 & 4.5 \\
\hline $\mathrm{CCl}_{4}$ & McCool et al. (226) $^{-1}$ & $283-328$ & $0.87-0.99$ & 0.575 & 145 & 6.3 \\
\hline $\mathrm{CH}_{3} \mathrm{~F}$ & Lang et al. ${ }^{(227)}$ & $153-440$ & $0.21-0.90$ & 0.405 & 56 & 8.5 \\
\hline $\mathrm{CHF}_{3}$ & Lang et al. (228) & $142-433$ & $0.31-0.99$ & 0.434 & 86 & 6.6 \\
\hline $\mathrm{CHCIF}_{2}$ & Vardag et al. ${ }^{(229)}$ & $147-383$ & $0.49-1.04$ & 0.461 & 164 & 6.8 \\
\hline $\mathrm{CF}_{3} \mathrm{Cl}$ & Has et al..$^{(230)}$ & $133-433$ & $0.45-0.99$ & 0.473 & 181 & 8.0 \\
\hline $\mathrm{CF}_{3} \mathrm{Br}$ & Has et al. ${ }^{(230)}$ & $141-432$ & $0.44-0.99$ & 0.492 & 191 & 5.9 \\
\hline
\end{tabular}

TABLE 6. Parameters of the rough hard sphere model for a series of halomethanes derived from the self-diffusion data. For details see the original references

\begin{tabular}{|c|c|c|c|c|}
\hline Substance & Reference & $T$-range $(\mathrm{K})$ & $A$ & $\sigma\left(10^{-10} \mathrm{~m}\right)$ \\
\hline $\mathrm{CHCl}_{3}$ & De Zwaan et al. ${ }^{(232)}$ & $341-460$ & $0.64 \pm 0.01$ & $4.97 \pm 0.06$ \\
\hline $\mathrm{CHCl}_{3}$ & Harris et al..$^{(231)}$ & $278-348$ & $0.76 \pm 0.02$ & $4.85 \pm 0.03$ \\
\hline $\mathrm{CHCl}_{3}$ & Prielmeier et al. ${ }^{(225)}$ & $233-397$ & $0.4-0.8$ & $4.84 \pm 0.05$ \\
\hline $\mathrm{CH}_{3} \mathrm{Cl}$ & Prielmeier et al. ${ }^{(225)}$ & $210-440$ & $0.4-0.75$ & $3.98 \pm 0.03$ \\
\hline $\mathrm{CH}_{2} \mathrm{Cl}_{2}$ & Prielmeier et al. ${ }^{(225)}$ & $186-406$ & $0.3-0.8$ & $4.43 \pm 0.04$ \\
\hline $\mathrm{CH}_{3} \mathrm{~F}$ & Lang et al. $(227)$ & $153-440$ & $0.4-1.0$ & $3.57 \pm 0.04$ \\
\hline $\mathrm{CH}_{3} \mathrm{H}$ & Lang et al. ${ }^{(228)}$ & $142-433$ & $0.3-1.0$ & $3.95 \pm 0.04$ \\
\hline $\mathrm{CHCIF}_{2}$ & Vardag et al. (229) & $147-383$ & $0.4-0.8$ & $4.32 \pm 0.01$ \\
\hline $\mathrm{CF}_{3} \mathrm{Cl}$ & Has et al..$^{(230)}$ & $133-433$ & $0.45-0.85$ & $4.8 \pm 0.1$ \\
\hline $\mathrm{CF}_{3} \mathrm{Br}$ & Has et al..$^{(230)}$ & $141-432$ & $0.68-0.75$ & $5.1 \pm 0.1$ \\
\hline
\end{tabular}


of the real molecules. It thus remains to be determined whether or not the parameters derived in the limited $\rho, T$-range permit a reliable extrapolation into regions not covered by the data.

\subsection{Larger Alkanes}

Besides the halomethanes the hydrocarbons comprise a relatively well studied group of compounds. The intensive investigation of these substances was, no doubt, motivated by their occurrence in oil and their widespread use in industry.

Table 7 gives the substances studied at elevated pressures. Figure 22 gives the $A$ parameter values derived from fitting the self diffusion data obtained by Jonas and his group ${ }^{(233)}$ to the RHS model. They clearly show a significant temperature dependence. These data represent an early example of the approximate nature of this model and in our opinion a clear indication for the necessity to include attractive interactions into the analysis. The data obtained from fitting the RHS- and IS-model to all $n$-alkanes studied, provided the availability of sufficient $p V T$-data, are compiled in Tables 8 and 9.

It is remarkable that most of these compounds can be described with reasonable accuracy by the interacting sphere model. Only butane and a polyethylene wax with an average chain length of 154

TABLE 7. Self-diffusion studies of larger aliphatic hydrocarbons

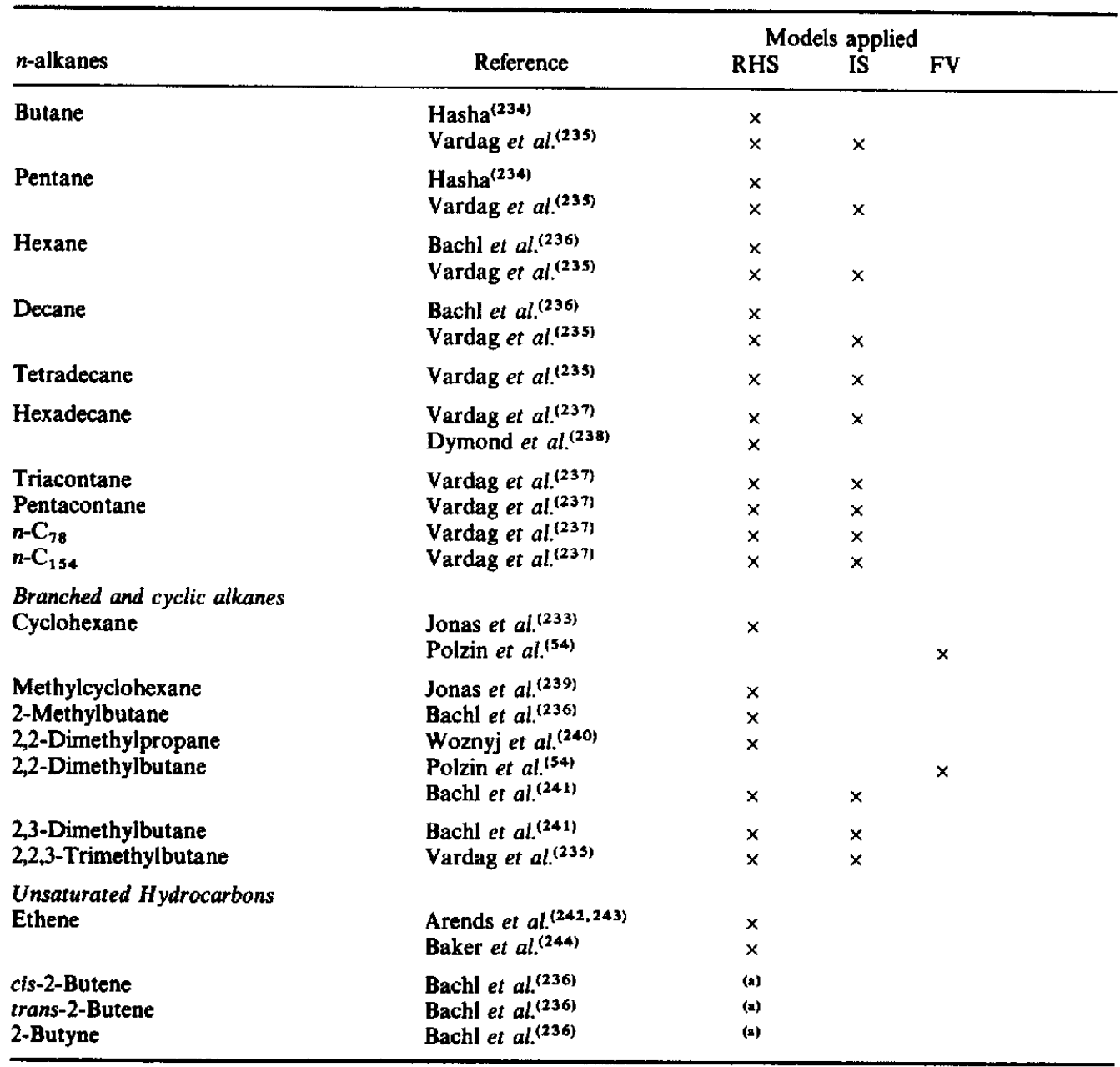

(a) No evaluation possible, no $p V T$-data available. 


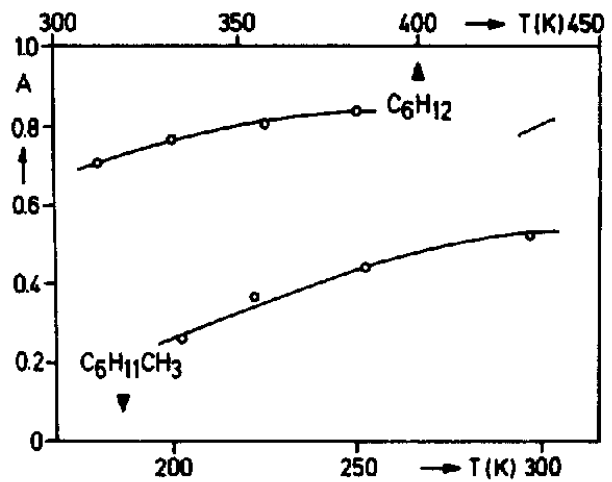

FIG. 22. The parameter $A$ for self-diffusion in cyclohexane and methylcyclohexane as derived by Jonas $e t$ al. ${ }^{(233)}$ from the fit to the RHS-model at different temperatures.

TABLE 8. Parameters of the interacting sphere model for several alkanes from saturation vapor pressure to $200 \mathrm{MPa}$

\begin{tabular}{|c|c|c|c|c|c|}
\hline Substance & Reference & $T$-range (K) & $\sigma_{L}\left[10^{-10} \mathrm{~m}\right]$ & $\varepsilon / k_{\mathrm{B}}[\mathrm{K}]$ & $\%$ Std. dev. \\
\hline \multicolumn{6}{|l|}{ n-alkanes } \\
\hline $\mathrm{C}_{4} \mathrm{H}_{10}$ & Vardag et al. ${ }^{(235)}$ & $150-451$ & 5.26 & 226 & 9.4 \\
\hline $\mathrm{C}_{9} \mathrm{H}_{12}$ & Vardag et al. ${ }^{(235)}$ & $174-451$ & 5.70 & 186 & 4.7 \\
\hline $\mathrm{C}_{6} \mathrm{H}_{14}$ & Vardag et al. ${ }^{(233)}$ & $214-443$ & 5.97 & 242 & 5.5 \\
\hline $\mathrm{C}_{10} \mathrm{H}_{22}$ & Vardag et al. ${ }^{(235)}$ & $265-449$ & 6.98 & 379 & 2.3 \\
\hline $\mathrm{C}_{14} \mathrm{H}_{30}$ & Vardag et al. ${ }^{(233)}$ & $293-442$ & 7.61 & 679 & 4.8 \\
\hline $\mathrm{C}_{16} \mathrm{H}_{34}$ & Vardag et al. ${ }^{[237]}$ & $312-473$ & 8.09 & 576 & 3.9 \\
\hline $\mathrm{C}_{30} \mathrm{H}_{62}$ & Vardag et al. ${ }^{(237)}$ & $357-472$ & 9.77 & 1139 & 2.9 \\
\hline $\mathrm{C}_{30} \mathrm{H}_{102}$ & Vardag et al. ${ }^{[237]}$ & $390-476$ & 11.49 & 1715 & 2.6 \\
\hline$C_{78}$ & Vardag et al. ${ }^{(237)}$ & $397-476$ & 13.21 & 2448 & 3.7 \\
\hline$C_{154}$ & Vardag et al. ${ }^{(237]}$ & $408-476$ & 16.34 & 3420 & 8.9 \\
\hline \multicolumn{6}{|c|}{ branched alkanes } \\
\hline 2,3-DMB ${ }^{(\mathbf{a})}$ & Bachl et al..$^{(241)}$ & $252-453$ & 6.08 & 197 & 4.8 \\
\hline 2,2-DMB ${ }^{(2)}$ & Bachl et al. ${ }^{(241)}$ & $250-450$ & 6.05 & 303 & 5.3 \\
\hline $2,2,3-\mathrm{TMB}^{(\mathrm{b})}$ & Vardag et al. ${ }^{(237)}$ & $296-450$ & 6.33 & 340 & 11.5 \\
\hline
\end{tabular}

(a) DMB = Dimethylbutane.

(b) $\mathrm{TMB}=$ Trimethylbutane.

carbon atoms reveal large and very systematic standard deviations. Also the fit for pentane and hexane is fairly poor. All alkanes deviate strongly from spherical geometry. The $n$-alkanes with a chain length between C-4 and C-6 yield the worst results. An inspection of mechanical models reveals that for these substances the deviation from spherical symmetry is larger than for ethane and propane.

For the longer $n$-alkanes, dynamic trans-gauche equilibria lead to the formation of more spherical random coils. Thus the description by spherical approximations becomes more precise. For the longest alkanes, with an average chain length of more than 50 carbon atoms, the coils of the single molecules could begin to intertwine. This in our opinion is most clearly seen in the low $A$-parameter found for these three compounds, although even the C-154 alkane is too short for the reptation model $^{(245)}$ to be applicable.

A further check for the qualitative conclusion given above is provided by the diffusion data of the methylated butane derivatives given in Tables 8 and 9 . These compounds show large systematic standard deviations in the fit parameters for the interacting sphere model. The introduction of the 
TABLE 9. Parameters of the rough hard sphere model for a series $n$-alkanes derived from fitting the self diffusion. For details see the original references

\begin{tabular}{|c|c|c|c|c|}
\hline Substance & Reference & $T$-range $[\mathrm{K}]$ & $A$ & $\sigma\left(10^{-10} \mathrm{~m}\right)$ \\
\hline \multicolumn{5}{|l|}{ n-alkanes } \\
\hline $\mathrm{CH}_{4}$ & Greiner-Schmid et al. ${ }^{(220)}$ & $110-454$ & $0.93-1.07$ & $3.5 \pm 0.15$ \\
\hline $\mathrm{C}_{2} \mathrm{H}_{6}$ & Greiner-Schmid et al. ${ }^{(20)}$ & $136-454$ & $0.80-0.97$ & $4.1 \pm 0.1$ \\
\hline $\mathrm{C}_{3} \mathrm{H}_{\mathrm{B}}$ & Greiner-Schmid et al. ${ }^{(20)}$ & $135-453$ & $0.37-0.93$ & $4.60 \pm 0.04$ \\
\hline $\mathrm{C}_{4} \mathrm{H}_{10}$ & Vardag et al. ${ }^{(235)}$ & $150-451$ & $0.61-0.99$ & $5.03 \pm 0.04$ \\
\hline $\mathrm{C}_{5} \mathrm{H}_{12}$ & Vardag et al. ${ }^{(235)}$ & $174-451$ & $0.55-0.98$ & $5.36 \pm 0.05$ \\
\hline $\mathrm{C}_{6} \mathrm{H}_{14}$ & Vardag et al. ${ }^{(235)}$ & 214443 & $0.61-0.85$ & $5.65 \pm 0.03$ \\
\hline $\mathrm{C}_{10} \mathrm{H}_{22}$ & Vardag et al. ${ }^{(235)}$ & $265-449$ & $0.47-0.68$ & $6.68 \pm 0.05$ \\
\hline $\mathrm{C}_{14} \mathrm{H}_{30}$ & Vardag et al. ${ }^{(235)}$ & $293-442$ & $0.27-0.48$ & $7.38 \pm 0.02$ \\
\hline $\mathrm{C}_{16} \mathrm{H}_{34}$ & Vardag et al. ${ }^{(237)}$ & $312-473$ & $0.33-0.64$ & $7.77 \pm 0.05$ \\
\hline $\mathrm{C}_{30} \mathrm{H}_{62}$ & Vardag et al. ${ }^{(237)}$ & $357-472$ & $0.20-0.30$ & $9.53 \pm 0.04$ \\
\hline $\mathrm{C}_{50} \mathrm{H}_{102}$ & Vardag et al. ${ }^{(237)}$ & $390-476$ & $0.11-0.16$ & $11.28 \pm 0.03$ \\
\hline$C_{78}$ & Vardag et al. ${ }^{(237)}$ & $397-476$ & $0.04-0.08$ & $13.00+0.1$ \\
\hline $\mathrm{C}_{154}$ & Vardag et al. ${ }^{(237)}$ & $408-476$ & $0.02-0.03$ & $16.15 \pm 0.04$ \\
\hline \multicolumn{5}{|l|}{ branched alkanes } \\
\hline 2,3-DMB ${ }^{(0)}$ & Bachl et al..$^{(241)}$ & $252-453$ & $0.62-0.93$ & $5.68 \pm 0.04$ \\
\hline 2,2-DMB & Bachl et al. ${ }^{(241)}$ & $250-450$ & $0.50-0.74$ & $5.74 \pm 0.05$ \\
\hline 2,2,3-TMB ${ }^{(\mathbf{t})}$ & Vardag et al. ${ }^{(235)}$ & $296-450$ & $0.68-0.77$ & $6.04 \pm 0.02$ \\
\hline
\end{tabular}

(a) $\mathrm{DMB}=$ Dimethylbutane.

(b) $\mathrm{TMB}=$ Trimethylbutane.

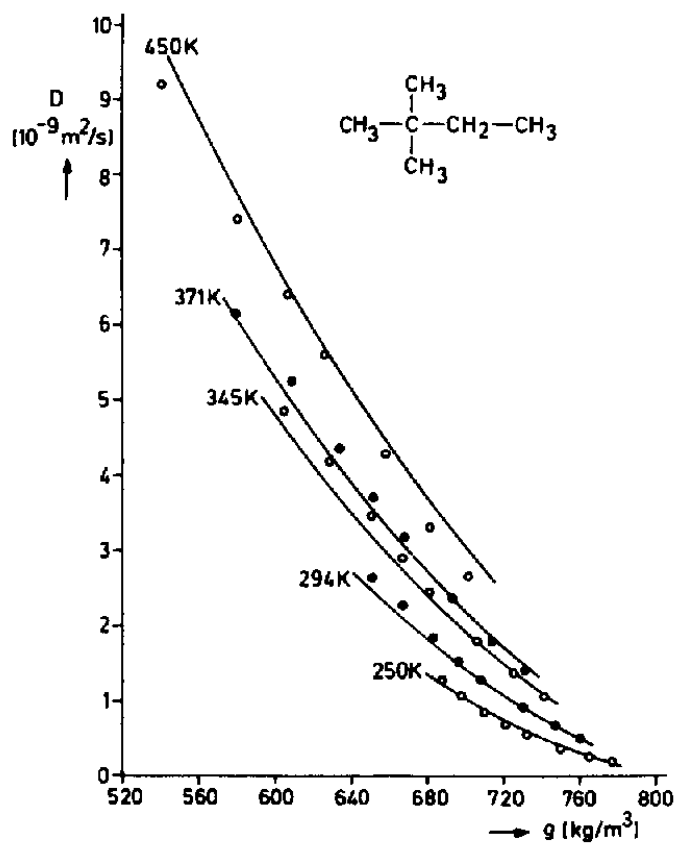

Fig. 23. Fit of the self-diffusion coefficient $D$ of 2,2 -dimethylbutane as function of the density $\rho^{(241)}$ to the interacting spheres model.

additional methyl groups leads to greater molecular asymmetry and to a steric restriction of the compounds to the conformers with the maximum number of trans methyl/methyl group contacts.

In Fig. 23 the interacting sphere fit for 2,2-dimethylbutane is given. The large and systematic deviations seen are indicative, in our opinion, of the failure of this simple model. In trying to specify 
the quality of the fits as mentioned previously, one is often limited by the available $p V T$-data. At the high density side of the fitting range, an error of $2 \%$ in the density may lead to $20 \%$ deviation of the calculated self diffusion values. In order to eliminate this source of error, we have set up a high pressure apparatus for density measurements. The results given for 2,2-dimethylbutane and 2,3-dimethylbutane were the first data obtained with this instrument. The $p, V, T$-data are judged reliable to $\pm 0.5 \%$ and corroborate the conclusions drawn.

At the moment, a precise check of the two models discussed here must be postponed until more $p V T$-data become available. The applicability of this treatment for the extrapolation of the limited data sets to regions of the $p, T$-space not covered by experiment can only be tested if sufficiently precise $p, V, T$-data have been collected for a strategically chosen set of substances.

The polyethylene waxes with an average chain length of 78 and 154 carbons respectively provide a first insight into the dynamics of polymer melts in this series. The viscoelastic properties of molten linear polymers are usually well described by the Rouse-(246) or reptation-models. ${ }^{(245,247,248)}$ Critical tests of these models could only be achieved if the measurements were to include values from polyethylenes with a considerably higher molecular weight. Beyond the critical molecular weight $M_{c}$, entanglements, typical of long linear random coil molecules in the reptation model, hinder significantly the translational mobility. $M_{\mathrm{c}}$ was derived for polyethylene to $3800<M_{\mathrm{c}}<5600$, beyond the range covered in these experiments. All data collected here should be describable by the Rouse model, which demands $D \sim M_{\mathrm{w}}^{-1}$ while for reptation $D \sim M_{\mathrm{w}}^{-2}$ is expected. Figure 24 gives a $\log D$ versus $\log M_{\mathrm{w}}$ plot showing the isobars of $D$ for all alkanes studied here at $473 \mathrm{~K}$. The isobars are linear, with the slopes increasing with pressure.

Although all these alkanes are in the lower molecular weight range, where Rouse dynamics should apply, $D$ is found to be proportional to $M \vec{w}^{-2 \pm 0.2}$. The exponent appears to increase significantly with pressure. In Fig. 25 the saturated vapour pressure diffusion data for $n$-alkanes with 5 or more carbons are collected. Even the inclusion of these small molecules retains the $M_{\mathrm{w}}^{-2 \pm 0.2}$ dependence of $D$. Similar observations have been discussed in the literature ${ }^{(249.250)}$ and are usually explained by the influence of the free volume upon the translational mobility. The graphs of Figs 24 and 25 obviously provide an excellent method for the interpolation of self diffusion coefficients of alkanes with

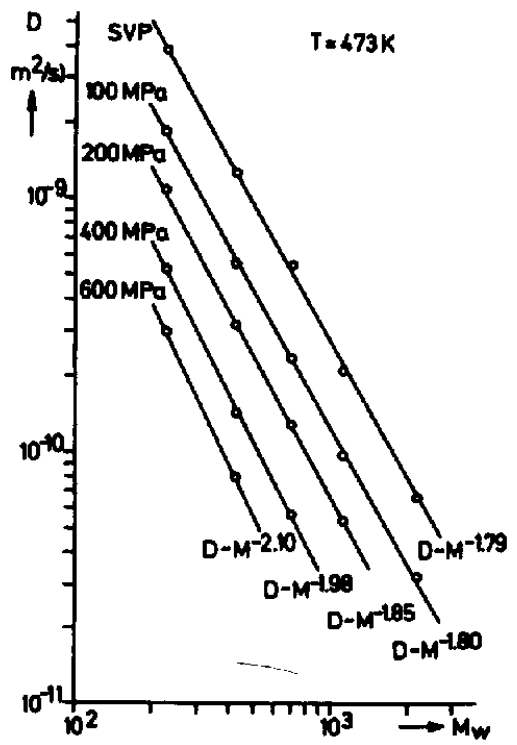

FIG. 24. Log/log plot of the isobars of the self-diffusion coefficients $D$ of $n$-alkanes and polyethylene waxes versus the molecular weight $M_{w} \cdot{ }^{(237)}$ 


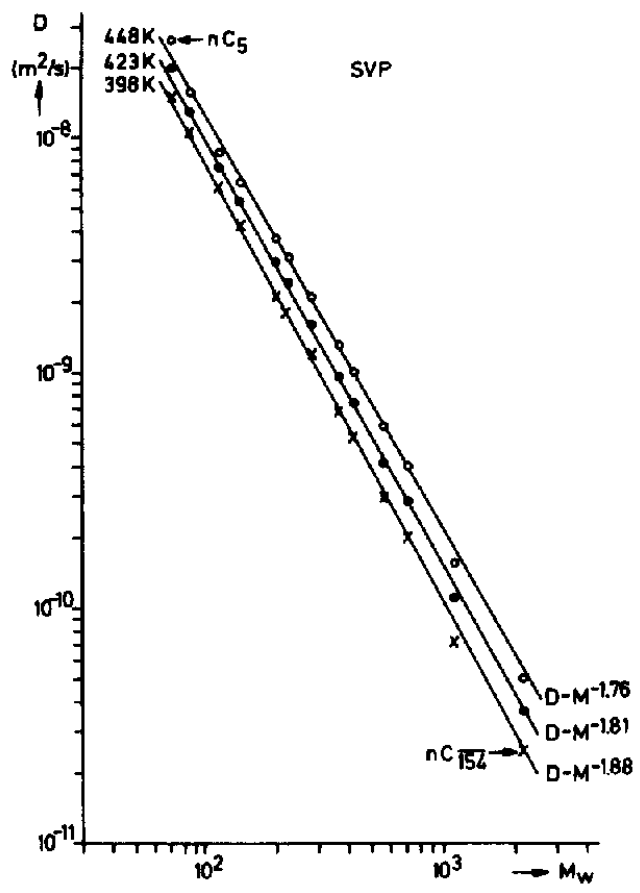

Fig. 25. $\log / \log$ plot of the saturation vapour pressure self-diffusion coefficients $D$ of $n$-alkanes versus the molecular weight $M_{\mathbf{w}}{ }^{(237)}$

intermediate chain lengths. The physical analysis of the functional form of the molecular weight dependence must await studies on longer polyethylenes.

\subsection{Miscellaneous Non-Associating Liquids}

The substances investigated are given in Table 10 together with the liquid state models applied for their description. The self diffusion of benzene has been determined very carefully both by NMR and tracer techniques. ${ }^{(51,226)}$ The data are sufficiently precise to be used for the gradient calibration in spin echo experiments. The chemical stability, the simplicity of the proton and carbon-13 NMR spectrum, and the availability of very pure benzene further support this proposal. Polzin et al. ${ }^{(54)}$ described the $p, T$-dependence of the self diffusion coefficient to better than $\pm 2 \%$ by the empirical polynomial proposed by Collings et al. ${ }^{(51)}$

$$
\begin{aligned}
\ln \left(D 10^{-9} \mathrm{~m}^{2} \mathrm{~s}^{-1}\right)= & 5.953-1550.97(T / \mathrm{K})^{-1}-26.035(T / \mathrm{K})^{-2}-1.108\left(p / \mathrm{MPa}(T / \mathrm{K})^{-1}\right) \\
& -368.2 \times 10^{-5}(p / \mathrm{MPa})+8.9064 \times 10^{-6}(\mathrm{p} / \mathrm{MPa})^{2}+17.565 \times 10^{-12}(\mathrm{p} / \mathrm{MPa})^{-3} .
\end{aligned}
$$

2-Ethylhexylbenzoate is the most viscous non-associating liquid analyzed by the RHS model. ${ }^{(251)}$ Only the high temperature isotherms were evaluated leading to an $A$-value between 0.17 to 0.24 and a RHS-diameter that decreased from $0.734 \mathrm{~nm}$ at $313 \mathrm{~K}$ to $0.718 \mathrm{~nm}$ at $373 \mathrm{~K}$.

The isobars of the self diffusion coefficient for hexamethylcyclotrisilazane (HMCTS) and octamethylcyclotetrasiloxane (OMCTS) could be followed in the deeply supercooled range. In Arrhenius-plots, all isobars reveal a curvature that becomes most pronounced below the melting temperature. The isobars were fitted to the VTF-equation. The parameters obtained are collected in Table 11. They show a strong increase of the ideal glass transition temperature $T_{0}$ with pressure.

In Fig. 27 the experimental points and the lines of best fit are given for HMCTS. It is interesting to note, that the equilibrium crystallization of this almost spherical non-polar compound occurs at all 
TABLE 10. Self-diffusion studies by NMR on miscellaneous non-associating liquids

\begin{tabular}{|c|c|c|c|c|}
\hline \multirow[b]{2}{*}{ n-alkanes } & \multirow[b]{2}{*}{ Reference } & \multicolumn{3}{|c|}{ Models applied } \\
\hline & & RHS & VTF & FV \\
\hline Benzene & $\begin{array}{l}\text { Parkhurst et al. (252) } \\
\text { Polzin et al. } .^{(34)}\end{array}$ & $x$ & & $x$ \\
\hline Hexadeuterobenzene & Parkhurst et al. ${ }^{(252)}$ & $x$ & & \\
\hline Trimethylbenzene & Polzin et al. ${ }^{(54)}$ & & & $x$ \\
\hline Hexafluorobenzene & Hogenboom et al. ${ }^{(57)}$ & $x$ & & $x$ \\
\hline Pentafluorobenzonitrile & Polzin et al..$^{(54)}$ & & & $x$ \\
\hline Pyridine & Fury et al. ${ }^{(253)}$ & $x$ & & \\
\hline Perfluorocyclobutane & Finney et al..$^{(254)}$ & $x$ & & \\
\hline Dibromtetrafluoroethane & Polzin et al..$^{(54)}$ & & & $x$ \\
\hline 1,2-Dichloroethane & Malhotra et al. ${ }^{(25 s)}$ & $x$ & & \\
\hline Sulphurhexafluoride & De Zwaan et al. ${ }^{(356)}$ & $x$ & & \\
\hline Tetramethylsilane & Parkhurst et al. ${ }^{(252)}$ & $x$ & & \\
\hline Tetramethyltin & Polzin et al. ${ }^{(54)}$ & & & $x$ \\
\hline Octamethylcyclotetrasiloxane & Greiner-Schmid et al.(257) & & $x$ & \\
\hline Hexamethylcyclotrisilazane & Greiner-Schmid et al. ${ }^{(257)}$ & & $x$ & \\
\hline 2,2-Dimethylpropionitrile & Woznyj et al. ${ }^{(240)}$ & & & \\
\hline 2-Ethylhexylbenzoate & Walker et al. ${ }^{(231)}$ & $x$ & & \\
\hline Carbon dioxide & Krynicki et al. (258) & & & \\
\hline Hydrogen chloride/ & Krynicki et al. ${ }^{(259)}$ & & & \\
\hline Deuterium chloride & & & & \\
\hline
\end{tabular}

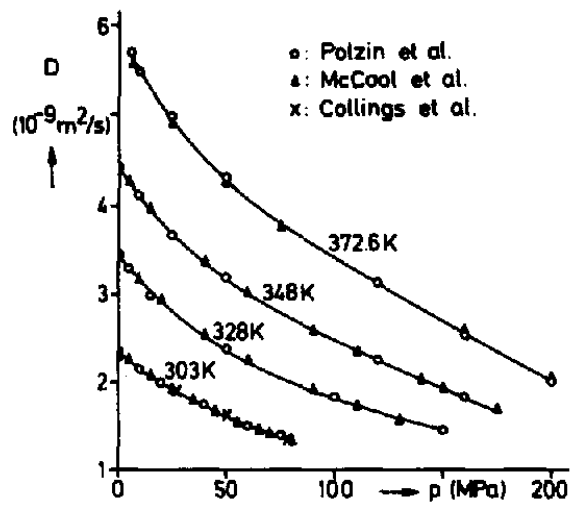

FIg. 26. Comparison of the isotherms of the self-diffusion coefficient $D$ of benzene of obtained by various authors. $^{(34,226,51)}$

TABLE 11. Fit parameters $T_{0}, D_{0}$ and $B$ of the VTF equation ${ }^{(257)}$ for HMCTS and OMCTS

\begin{tabular}{lcccccc}
\hline$p[\mathrm{MPa}]$ & 0.1 & 25 & 50 & 100 & 150 & 200 \\
\hline Substance: HMCTS & & & & & & \\
$T_{0}[\mathrm{~K}]$ & 102 & - & 133 & 159 & 162 & 159 \\
$D_{\circ}\left[10^{-9} \mathrm{~m}^{2} / \mathrm{S}\right]$ & 44.2 & - & 17.2 & 9.2 & 8.06 & 8.68 \\
$B[\mathrm{~K}]$ & 858 & - & 669 & 554 & 601 & 703 \\
Substance: $:$ OMCTS & & & & & & \\
$T_{\circ}[\mathrm{K}]$ & 52 & 81 & 103 & 165 & 199 & 237 \\
$D_{\circ}\left[10^{-9} \mathrm{~m}^{2} / \mathrm{S}\right]$ & 81.3 & 31.3 & 16.6 & 4.63 & 2.42 & 1.32 \\
$B[\mathrm{~K}]$ & 1311 & 1049 & 893 & 537 & 417 & 291 \\
\hline
\end{tabular}




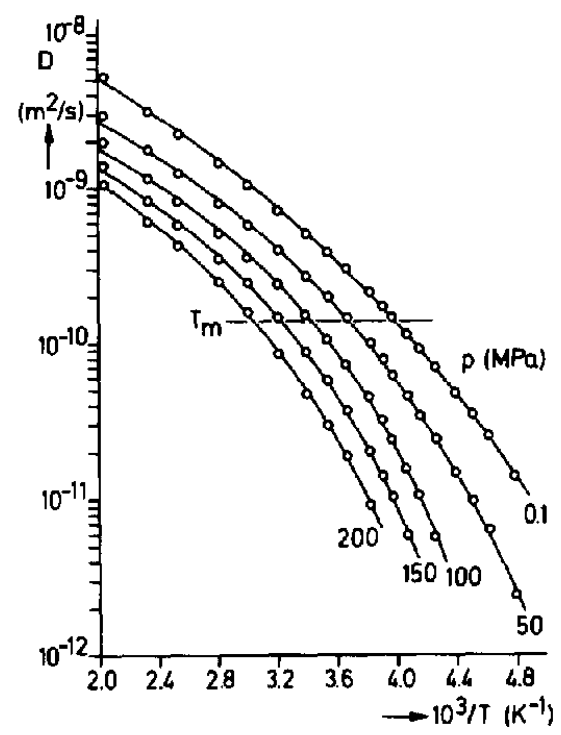

FIG. 27. Isobars of the self-diffusion coefficient $D$ of hexamethylcyclotrisilazane extending deeply into the supercooled range.

pressures at identical values of the self-diffusion coefficient (horizontal line in Fig. 27). A similar observation can be made for OMCTS.

Carbon dioxide, ${ }^{(258)}$ deuterium chloride, hydrogen chloride, ${ }^{(259)}$ and chlorotrifluoromethane ${ }^{(218)}$ were studied in the region of their critical points. The NMR studies on all four compounds conclude that a critical anomaly of the self-diffusion coefficient cannot be observed. This is in contrast to the well established anomalies in the thermal conductivity and shear viscosity. This is also in contradiction to earlier diffusion studies on carbon dioxide by the capillary technique.

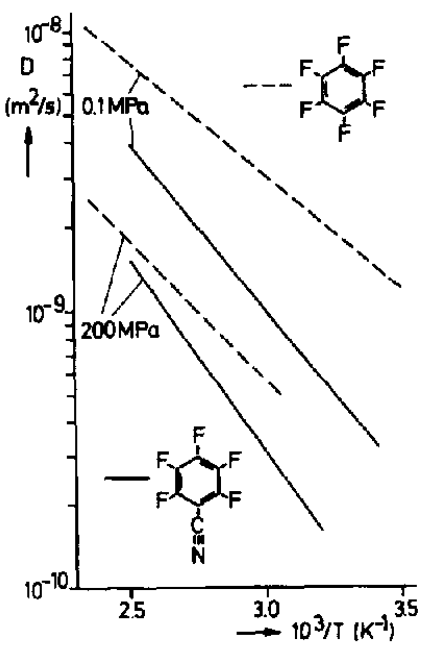

FIG. 28. Isobars of the self-diffusion coefficient $D$ of hexafluorobenzene ${ }^{(57)}$ and pentafluorobenzonitrile ${ }^{(54)}$ reveal- $^{2}$ ing the retarding influence of the large dipole moment of the nitrile group. 


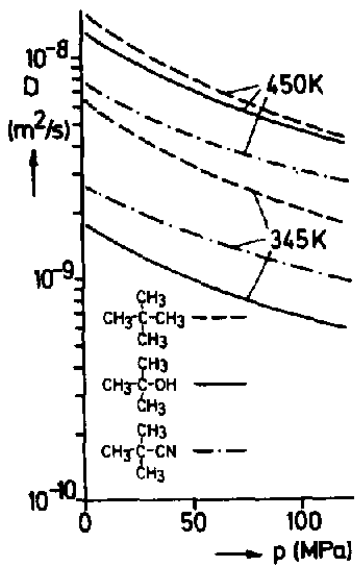

FIG. 29. Isotherms of the self-diffusion coefficient $D$ in three 2-methylpropane derivatives. ${ }^{(240)}$

The comparison of the self diffusion coefficients found for hexafluorobenzene ${ }^{(57)}$ and pentafluorobenzonitrile ${ }^{(54)}$ and 2,2-dimethylpropane and 2,2-dimethylpropionitrile ${ }^{(240)}$ show very clearly the retarding influence of large dipole moments upon the translational mobility. The volume and shape of these two pairs of molecules are very similar. The dipole moment of the nitrile group, which is around 4 Debye for these two substances, retards the translational mobility by at least a factor of two, especially at high temperatures. Similar influences may be seen for the halomethanes as have been discussed above. However, the dipole moments of these compounds are only around 2 Debye and thus this effect is less pronounced.

Figure 28 gives the Arrhenius plot of the pair of aromatic compounds. Two isotherms for the aliphatic molecules are presented in Fig. 29: two isobars of the monohydric alcohol 2-methyl2-propanol are also included. The comparison shows that, at low temperatures, the retarding influence of the hydrogen bonds is more important than that of the large dipole moment. At $450 \mathrm{~K}$ however, 2,2-dimethylpropane and the alcohol have almost identical mobilities. The influence of hydrogen bonding on self diffusion will be discussed in the next section.

\subsection{Hydrogen Bonded Liquids}

The lower alcohols and water are the only hydrogen bonded liquids for which self diffusion has been studied at elevated pressures (Table 12). Two isotherms of 2-methyl-2-propanol are given in Fig. 29.

TABLE 12. High pressure NMR studies of the self-diffusion coefficient of hydrogen bonded liquids

Water

\begin{tabular}{|c|c|}
\hline${ }^{1} \mathrm{H}_{2}{ }^{16} \mathrm{O}$ & $\begin{array}{l}\text { Kiselnik et al.; }{ }^{(260)} \text { Woolf; } ;^{(261)} \text { Angell et al. } .^{(262)} \text { Krynicki et al. } ;^{(263)} \text { Woolf and } \\
\text { Harris,; } ;^{(264)} \text { Lamb et al.;(263) Prielmeier et al. } .^{(266)} \text { Prielmeier et al. }{ }^{(267)}\end{array}$ \\
\hline${ }^{1} \mathrm{H}_{2}{ }^{18} \mathrm{O}$ & Woolf and Harris ${ }^{(264)}$ \\
\hline $\mathrm{D}_{2} \mathrm{O}$ & Wilbur et al.; ${ }^{(268)}$ De Fries and Jonas; ${ }^{(269)}$ Prielmeier et al. ${ }^{(267)}$ \\
\hline $\mathrm{CH}_{3} \mathrm{OH}$ & Hurle et al.;(270) Karger et al..$^{(271)}$ \\
\hline $\mathrm{CH}_{3} \mathrm{OD}$ & Jonas and Akai; ${ }^{(272)}$ Hurle et al.; ${ }^{(270)}$ Karger et al. ${ }^{(271)}$ \\
\hline $\mathrm{C}_{2} \mathrm{H}_{5} \mathrm{OH}$ & Meckl and Zeidler; ${ }^{(273)}$ Karger et $a l^{(271)}$ \\
\hline$n-\mathrm{C}_{3} \mathrm{H}_{7} \mathrm{OH}$ & Meckl and Zejdler ${ }^{(273)}$ \\
\hline$\left(\mathrm{CH}_{3}\right)_{3} \mathrm{COH}$ & Woznyj et al. ${ }^{(240)}$ \\
\hline
\end{tabular}


They show that the retarding influence of this interaction is most clearly seen at low temperatures. All alcohols investigated hitherto can be well described by the rough hard sphere model, with a strongly temperature dependent $\boldsymbol{A}$-parameter. In the supercooled range this parameter becomes smaller than 0.01 . The hard sphere diameters obtained from the fitting agree reasonably well with diameters calculated from the van der Waals volumes and melting point densities. The isobaric temperature dependence of self diffusion in the neat alcohols is well characterized by the VTF-equation. The coefficients determined are compiled in Table 13.

In order to study the influence of isotopic substitution on translational mobility, the self-diffusion coefficients $(D)$ of methanol and partially deuterated methanol $\left(\mathrm{CH}_{3} \mathrm{OD}\right)$ were measured over the same wide temperature range. Figure 30 gives the isotherms for methanol-OH. Figure 31 gives the ratios $D_{\mathrm{r}}$ for two isobars from $150-450 \mathrm{~K}$. The ratios reveal a pronounced temperature dependence, especially in the supercooled region.

At the lowest temperature, $D_{\mathrm{r}}$ reaches a maximum value of 1.4 . This cannot be attributed to differences in molar volume, since for $\mathrm{CH}_{3} \mathrm{OH}$ and $\mathrm{CH}_{3} \mathrm{OD}$ this differs by $<1 \%$. According to kinetic theories for simple liquid dynamics, one would expect $D_{\mathrm{r}}$ to depend on the square root of the inverse mass ratio. For methanol and methan $\left({ }^{2} \mathrm{H}\right)$ ol this leads to a value of 1.016 , which cannot account for the differences in $D$.

The data for $D_{\mathrm{r}}$ presented by Hurle et al. ${ }^{(270)}$ between $283-313 \mathrm{~K}$ are in very good agreement with our results. Keeping in mind that there seems to exist a strong rotation-translation coupling in methanol, Hurle et al. ${ }^{(270)}$ compared their results for $D_{\mathrm{r}}$ with the inverse ratios of the square roots of the moments of inertia. However, the temperature dependence of $D_{r}$ as presented in Fig. 31 cannot be explained by this approach. It follows that the $p, T$-dependence of the mobility of the methanol molecules is not determined by the mechanics of the single free methanol molecule but is a collective property reflecting the dynamics of the hydrogen-bond network.

A similar temperature dependence of the isotope effect can be observed in other hydrogen-bonded liquids such as water. Most probably the observed temperature dependence of the isotope effect in methanol originates from the same effect. In liquid water this difference was assigned to the formation of a greater number of stronger hydrogen bonds in $\mathrm{D}_{2} \mathrm{O}$. Their influence should become more

TABLE 13. Fitted results (VTF equation) for methanol, methan $\left({ }^{2} H\right)$ ol, and ethanol

\begin{tabular}{lclll}
\hline & $p(\mathrm{MPa})$ & $T_{0}(\mathrm{~K})$ & $B(\mathrm{~K})$ & $B \times T_{0}^{-1}$ \\
\hline MeOH & 0.1 & 62 & 775 & 12.5 \\
& 50 & 64 & 800 & 12.5 \\
& 100 & 67.5 & 820 & 12.1 \\
& 150 & 69 & 850 & 12.3 \\
& 200 & 70 & 875 & 12.5 \\
MeOD & 250 & 71 & 900 & 12.7 \\
& 0.1 & 66 & 775 & 11.7 \\
& 50 & 68 & 800 & 11.8 \\
& 100 & 71.5 & 820 & 11.5 \\
& 150 & 73 & 850 & 11.6 \\
EtOH & 200 & 74 & 875 & 11.8 \\
& 250 & 75 & 900 & 12.0 \\
& 0.1 & 57 & 1000 & 17.5 \\
& 50 & 59.5 & 1040 & 17.5 \\
& 100 & 61.5 & 1050 & 17.1 \\
& 150 & 63.5 & 1080 & 17.0 \\
& 200 & 65 & 1110 & 17.1 \\
& 250 & 66 & 1135 & 17.2 \\
\hline
\end{tabular}




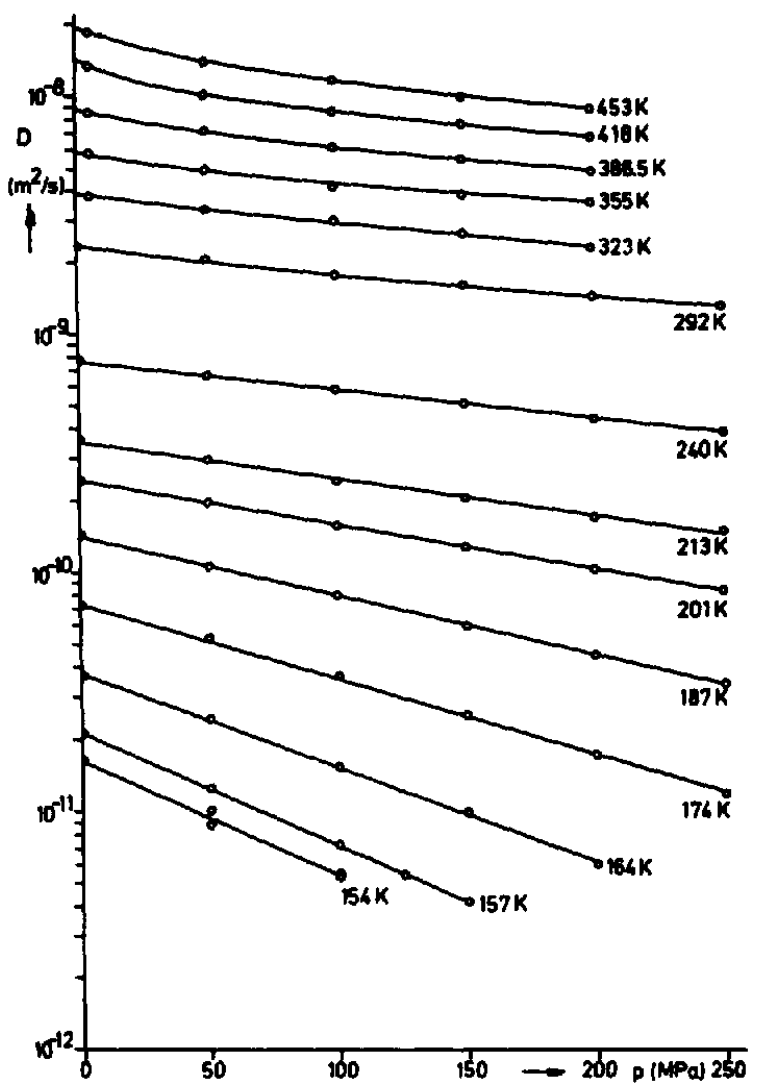

Fig. 30. Isotherms of the self-diffusion coefficient $D$ of liquid methanol- $(\mathrm{OH})^{(271)}$ as function of pressure $p$.

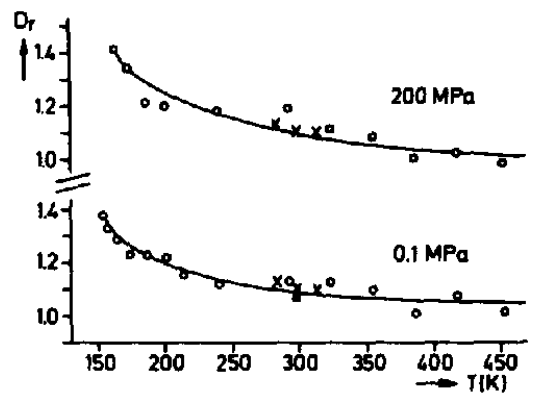

FIG. 31 Reduced self-diffusion coefficient $D_{r}=D\left(\mathrm{CH}_{3} \mathrm{OH}\right) /\left(D\left(\mathrm{CH}_{3} \mathrm{OD}\right)\right)$ for methanol and methanol-OD showing the temperature dependence of the dynamic isotope effect. ${ }^{(271)}$

pronounced at low temperatures. The corresponding effects observed in light and heavy water will be discussed later, together with the results derived for the rotational dynamics from spin-lattice relaxation measurements. Woolf and Harris ${ }^{(264)}$ studied the $p, T$-dependence of the dynamic isotope effect in light water, and light water containing oxygen-18, in the temperature range between $277 \mathrm{~K}$ to $333 \mathrm{~K}$ at pressures up to $300 \mathrm{MPa}$. In this region the ratio $\mathrm{D}\left(\mathrm{H}_{2}{ }^{16} \mathrm{O}\right) / \mathrm{D}\left(\mathrm{H}_{2}{ }^{18} \mathrm{O}\right)$ is independent of $p$ and $T$ and equal to the square root of the mass ratios. 
In Fig. 32 self-diffusion coefficients for $\mathrm{H}_{2} \mathrm{O}$ are given, extending from the supercritical range, where water can obviously be quantitatively described as a normal liquid with a binary collision model, ${ }^{\text {(265) }}$ down into the deeply supercooled range to $\sim 200 \mathrm{~K}$, where the temperature dependence of $\mathrm{D}$ becomes very steep. In the intermediate range around $350 \mathrm{~K}$, and below, the pressure dependence of $\mathrm{D}\left(\mathrm{H}_{2} \mathrm{O}\right)$

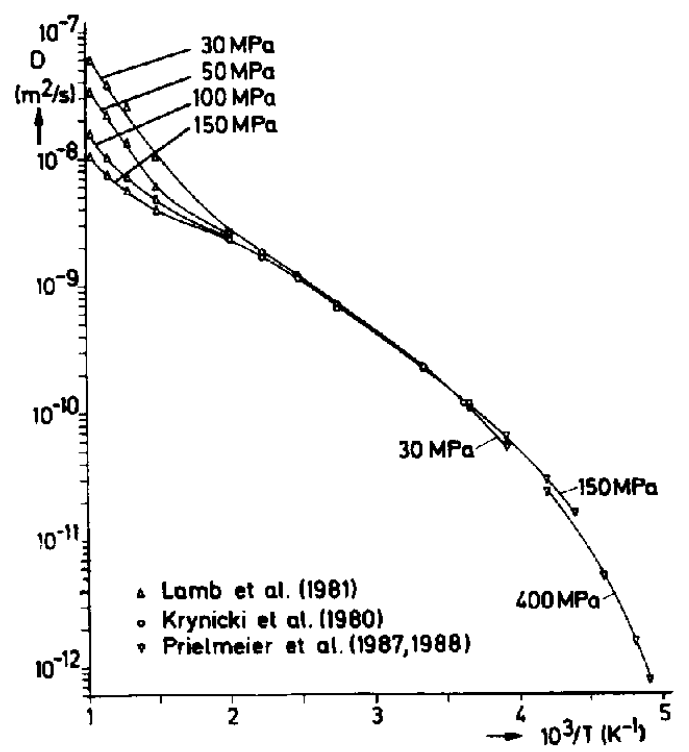

FIG. 32. Compilation of some isobars of the self-diffusion coefficients of liquid water.

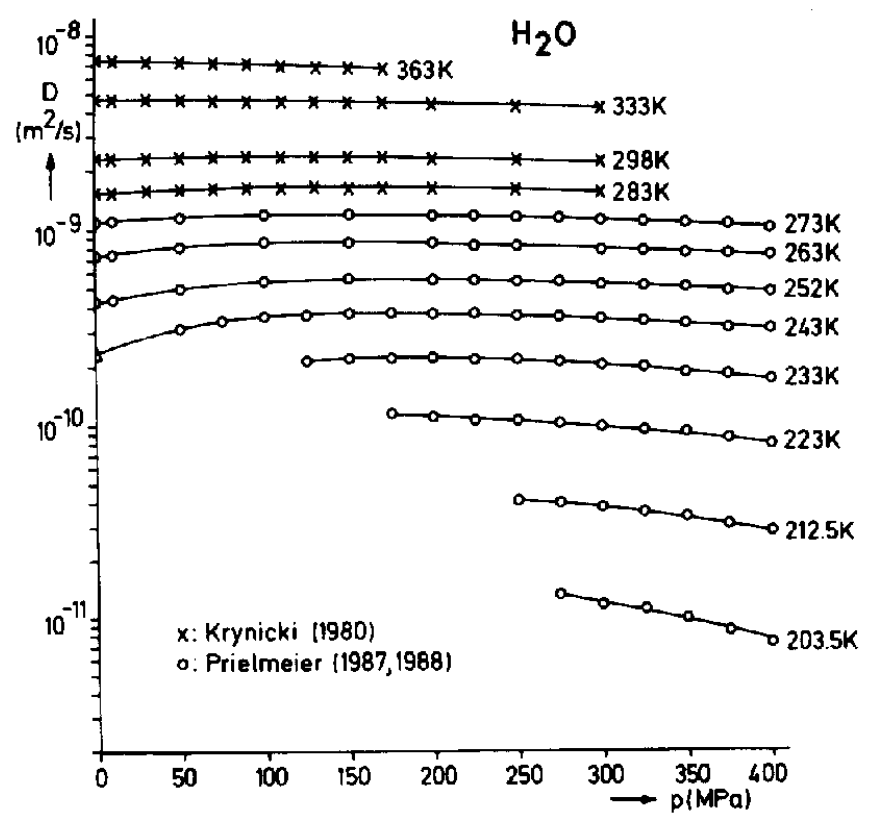

FIG. 33. Isotherms of the self-diffusion coefficients of liquid water showing the pressure anomaly in the temperature region between $273 \mathrm{~K}$ and $233 \mathrm{~K}$. 
becomes anomalous; increasing pressure facilitates translational and rotational mobility until at pressures around 100 to $200 \mathrm{MPa}$ a maximum of the mobility is reached. The effect becomes more pronounced in the supercooled region. Prielmeier et al. ${ }^{(267)}$ measured $\mathrm{D}\left(\mathrm{D}_{2} \mathrm{O}\right)$ for pressures up to $200 \mathrm{MPa}$ from $453 \mathrm{~K}$ down to $243 \mathrm{~K}$ into the metastable supercooled range (Fig. 33). The low temperature limit of these data was set by the short deuteron spin-spin relaxation times, which combined with the low self-diffusion coefficient do not permit a reliable determination of $D$ with the experimental setup used. The self diffusion results published in the more recent papers ${ }^{(261-267)}$ agree in the region of overlap within the precision claimed by the different groups. Weingärtner ${ }^{(35)}$ has recently critically reassessed all older data published.

\subsection{Binary Mixtures}

For the study of self-diffusion of the individual species in mixtures, Fourier transforming spin echoes obtained with either steady or pulsed gradients is the method of choice. However, relatively few have been studied hitherto and these have all been binary systems. They are compiled in Table 14.

The aqueous solutions will be discussed in detail together with the spin-lattice and spin-spin relaxation measurements in Section 5.5.3. The non-ionic solutions were studied in order to learn more about the pressure dependence of the biologically important effects of hydrophobic hydration and interaction. The peculiar pressure and concentration dependence observed for the alcohol as well as for the water molecules is demonstrated in Figs 34 and 35.

Careful and extensive studies of some binary mixtures of non-polar compounds have been published by Polzin and Weiss ${ }^{(54)}$ and Brüsewitz and Weiss. ${ }^{(278)}$ Attempts have been made to analyze the data in the framework of the free volume model, ${ }^{(56)}$ which demands that at constant volume

TABLE 14. Self diffusion in mixtures

\begin{tabular}{ll}
\hline Aqueous solutions & \\
$\mathrm{LiCl} / \mathrm{CsCl} / \mathrm{CaCl}_{2}$ in $\mathrm{D}_{2} \mathrm{O}$ & Akai et al. ${ }^{(274)}$ \\
$(\mathrm{CD})_{2} \mathrm{SO} / \mathrm{H}_{2} \mathrm{O}$ & Baker et al..$^{(275)}$ \\
$\left(\mathrm{CH}_{3}\right)_{3} \mathrm{COH} / \mathrm{H}_{2} \mathrm{O}$ & Woznyj $^{(276)}$ \\
$\left(\mathrm{CH}_{3}\right)_{3}\left(\mathrm{CH}_{2} \mathrm{OH}\right) / \mathrm{D}_{2} \mathrm{O}$ & Has $^{(277)}$ \\
$\mathrm{Sn}\left(\mathrm{CH}_{3}\right)_{4} / \mathrm{cyclohexane}$ & Polzin et al. \\
$n$-hexane/benzene & Brüsewitz et al. \\
methane/tetradecane & Vardag et al. \\
\hline
\end{tabular}

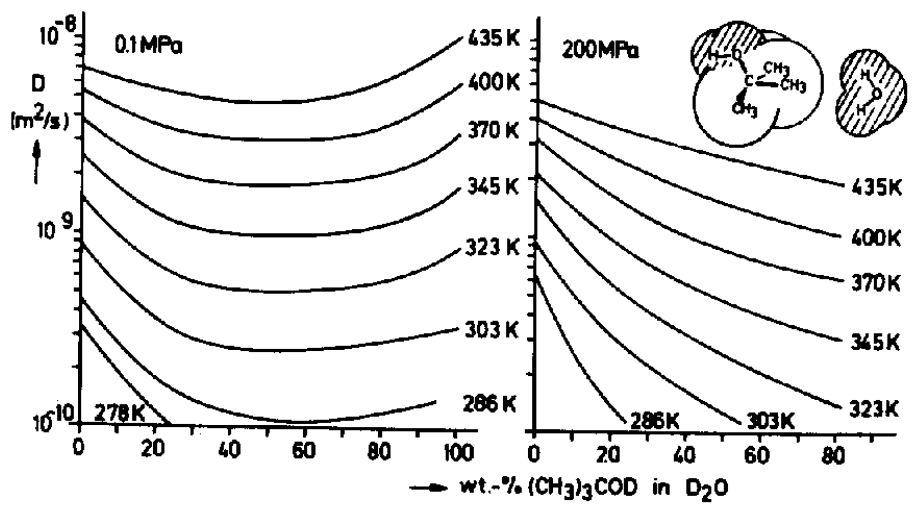

FIG. 34. Self-diffusion coefficient $D$ of methyl-2-propanol in aqueous solution as a function of composition at two pressures. ${ }^{(276)}$ 


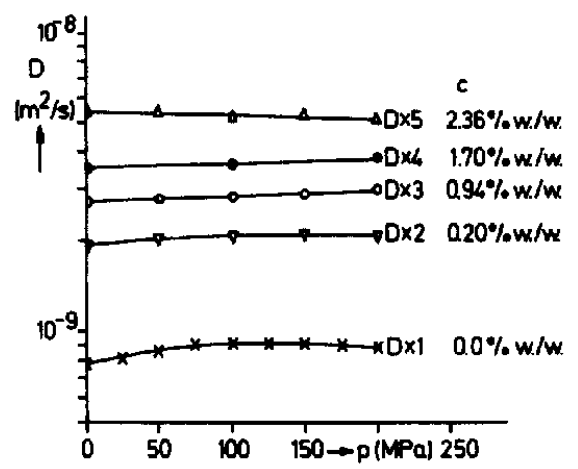

Fig. 35. Self-diffusion coefficients $D$ of the water molecules as function of pressure and concentration of 2,2dimethyl-1-propanol..$^{(277)}$

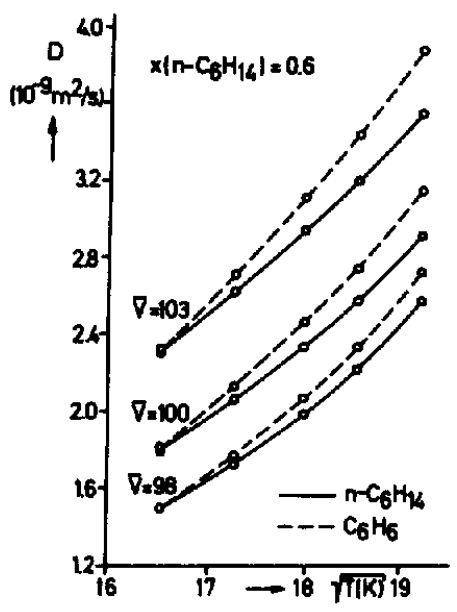

Fig. 36. Self-diffusion coefficients $D$ in $n$-hexane, benzene mixtures at constant density as a function of the square root of the absolute temperature. ${ }^{(278)}$

$D$ should depend linearly on $T^{1 / 2}$. The data for benzene/n-hexane, ${ }^{(279)}$ given in Fig. 36, give for all mixtures a definite curvature and prove that this model is not able to describe the results over a wider $p, T$-range.

\section{RELAXATION RATES: EXPERIMENTS}

\subsection{Atomic Liquids}

Reports of high pressure NMR relaxation experiments on atomic liquids are rather scarce. El-Hanany et al. ${ }^{(279)}$ and Warren et al. ${ }^{(28)}$ reported ${ }^{133} \mathrm{Cs}$ Knight shift and nuclear relaxation rate measurements on expanded liquid caesium over a density range of $1030\left(\mathrm{~kg} / \mathrm{m}^{3}\right)<\rho<1920\left(\mathrm{~kg} / \mathrm{m}^{3}\right)$. Conduction electron densities significantly lower than those of ordinary metals can be achieved before a metal-non-metal transition close to the liquid-gas critical point intervenes. The relaxation of the ${ }^{133} \mathrm{Cs}$ nuclei is dominated completely by the magnetic hyperfine coupling with conduction electrons. In this case the spin-lattice relaxation rate is governed by electron-spin fluctuations described by the 
low-frequency, transverse component of the imaginary part of the susceptibility $\chi^{\prime \prime}(q, \omega)$ :

$$
R_{1}=\left[(8 / 3)\left\langle|\Psi(0)|^{2}\right\rangle_{\mathrm{F}} \gamma_{\mathrm{N}} V_{\mathrm{A}}\right]^{2} \frac{k T}{\omega_{\mathrm{L}}} \int \mathrm{d} q q^{2} \chi_{+-}^{\prime \prime}\left(q, \omega_{\mathrm{L}}\right),
$$

with $\left\langle|\Psi|^{2}\right\rangle_{F}$ the probability amplitude at the nucleus averaged over all states at the Fermi surface, $\gamma_{N}$ the gyromagnetic ratio of the nucleus, and $\omega_{\mathrm{L}}$ the corresponding Larmor frequency. For noninteracting electrons the familiar Korringa rate $\mathrm{e}^{(2 \mathrm{~B} 1)}$ is obtained:

$$
\left(R_{1}\right)_{\text {Korr }}=(4 \pi / h)\left(\gamma_{\mathrm{N}} / \gamma_{\mathrm{e}}\right) k T K,
$$

with $K=(8 \pi / 3)\left\langle|\Psi(0)|^{2}\right\rangle_{F} V_{\wedge} \chi^{\prime}(0,0)$, the Knight shift. ${ }^{(84)}$

Generally, electron-electron interactions, ${ }^{(282)}$ or electron-ion scattering, ${ }^{(280)}$ alter the $q$-dependence of the volume susceptibility $\chi^{\prime \prime}\left(q, \omega_{\mathrm{L}}\right)$ resulting in a Korringa ratio

$$
\eta=R_{1} /\left(R_{1}\right)_{\text {Korr }} \neq 1 .
$$

With the usual random phase approximation (RPA) to $\mathrm{e}^{-}-\mathrm{e}^{-}$interactions, the Knight shift and the relaxation rates can be easily rationalized at high densities $\left(\rho \simeq 1900 \mathrm{~kg} / \mathrm{m}^{3}\right)$ close to the melting point. There the magnetic properties of liquid caesium correspond to an interacting electron gas. At reduced densities, however, a strong increase in both the Knight shift and the Korringa ratio appears which could not be understood within the RPA, hence a fundamental change in the $q$-dependent dynamic susceptibility must occur upon expanding the liquid metal (see Fig. 37). The authors could rule out the onset of a diffusive electron transport in the limit of strong electron-ion scattering. Rather they interpreted these effects as precursors of the metal-non-metal transition resulting from strong spin fluctuations towards a spin-density wave, or a metallic antiferromagnet above its ordering temperature.

Warren and Dupree ${ }^{(283)}$ investigated liquid Se by ${ }^{77} \mathrm{Se}$ NMR from the supercooled liquid (466 K) to the supercritical fluid $(1898 \mathrm{~K})$ and at pressures up to $79 \mathrm{MPa}$. Molten selenium forms a liquid with unique properties when considered in relation to the other liquid chalcogen elements $(\mathrm{O}, \mathrm{S}, \mathrm{Te}, \mathrm{Po})$. Liquid Se forms a liquid semiconductor in which the chemical bonding is purely covalent. Linearchain polymers are formed near $T_{m}$ because of the preservation of twofold coordination similar to those of sulphur above the polymerization temperature. Thus Se embodies both the low coordination and tendency for molecule formation (insulating liquid $\mathrm{O}_{2}, \mathrm{~S}_{8}$ ) and the electronic conduction properties of the heavier elements (metallic liquids $\mathrm{Te}, \mathrm{Po}$ ).

Over sufficiently wide ranges of temperature and pressure, including the supercritical fluid, radical modifications were expected to occur, accompanied by significant changes of the electronic structure
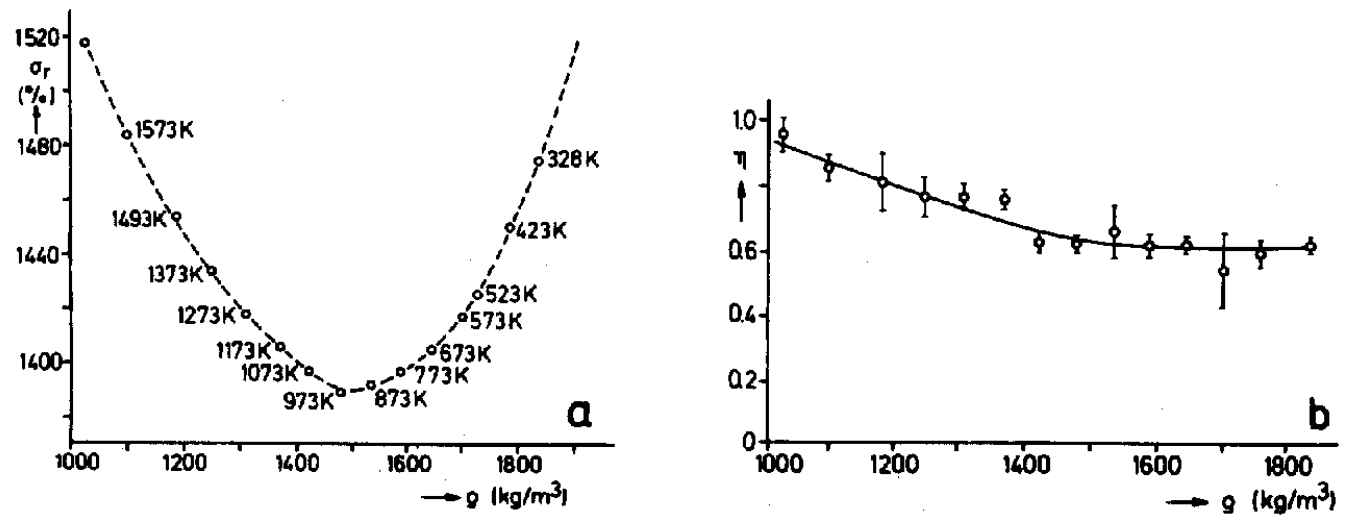

FiG. 37. (a) Variation of the ${ }^{133} \mathrm{Cs}$ Knight shift $\sigma_{\mathrm{r}}$ with density $\rho$ at various temperatures and constant pressure, $p=12 \mathrm{MPa}$. (b) Ratio $\eta$ of the ${ }^{133} \mathrm{Cs}$ nuclear relaxation rate to the Korringa rate versus density $\rho$ under isobaric conditions, $p=12 \mathrm{MPa} .^{(279,280)}$ 
and properties in the disordered liquid state. NMR provides a strong sensitivity to local static and dynamic magnetic fields. Hence nuclear relaxation rates are related to the microscopic electronic and structural dynamic properties. Nuclear spin-lattice $\left(1 / T_{1}\right)$, spin-spin $\left(1 / T_{2}\right)$ and spin-phase memory $\left(1 / T_{2}^{*}\right)$ relaxation rates were reported from the supercooled state at $466 \mathrm{~K}$ to the supercritical state at $1708 \mathrm{~K}$ and $41 \mathrm{MPa}$, and are shown in Fig. 38. Three regimes (I: $T<523 \mathrm{~K}$, II: $523 \mathrm{~K}<T<973 \mathrm{~K}$, III: $T>973 \mathrm{~K}$ ) with distinctly different relaxation characteristics were evident. In region I the spin-spin relaxation rate $1 / T_{2}$ decreased with temperature and increased sharply with frequency while the spin-lattice relaxation rate $1 / T_{1}$ increased with temperature and exhibited no frequency dependence. In region II both rates increased strongly with temperature. Furthermore $1 / T_{2}$ was independent of frequency whereas $1 / T_{1}$ increased considerably with decreasing frequency. Region III is the range studied in a high-pressure autoclave. Both relaxation rates were equal and exhibited a broad maximum at about $1273 \mathrm{~K}$. Data at $10 \mathrm{MPa}$ and $40 \mathrm{MPa}$ showed a negligible pressure dependence. The line broadening observed on cooling below $523 \mathrm{~K}$ was due to relaxation by the anisotropic chemical shielding (CSA) modulated by thermal reorientation of the molecular aggregates (chains and rings).

The data have been described with eqn (63) and eqn (64) taken in the slow motions limit $\left(\omega_{\mathbf{L}} \tau \gg 1\right)$. Average rotational correlation times could be obtained with the components of the chemical shielding tensor determined for crystalline trigonal $\mathrm{Se}^{(284)}$ yielding $\tau_{\mathrm{r}}(T=466 \mathrm{~K})=19.7 \pm 0.6(\mu \mathrm{s})$ and an apparent activation energy of $\Delta E_{\mathrm{r}}=85.8 \pm 9.6(\mathrm{~kJ} / \mathrm{mol})$. The spin-lattice relaxation, however, could not be explained with a CSA mechanism which yielded rates too small by a factor of approximately $10^{5}$. Rather it was due to a scalar interaction of the ${ }^{77} \mathrm{Se}$ nuclei with localized electron spins in dangling bonds at the chain ends. The scalar relaxation due to isotropic hyperfine couplings also provided the dominant relaxation mechanism for both $1 / T_{1}$ and $1 / T_{2}$ in region II. The corresponding relaxation rates are given by eqn (76) and eqn (77). Figure 38 indicates that the conditions $\left(\omega_{\mathrm{S}}-\omega_{\mathrm{L}}\right) \tau \ll 1$ and $\left(\omega_{\mathrm{S}}-\omega_{\mathrm{L}}\right) \tau \gtrsim 1$ hold above $1023 \mathrm{~K}$ and below $873 \mathrm{~K}$ respectively. The corresponding correlation time $\tau$ is the electron-electron exchange fluctuation time $\tau_{\mathrm{e}}$ which depends only on the average distance $\langle R\rangle \cong\left(n_{s}\right)^{-1 / 3}$ between the paramagnetic centres with no explicit dependence on temperature or pressure (see Figs $39 \mathrm{a}$ and $\mathrm{b}$ ). The spin density $n_{\mathrm{s}}$ increases with temperature and also very strongly with pressure at high temperatures due to bond cission. Thus application of pressure produces a sharp change in the magnetic properties as the paramagnetic centres delocalize. The strong dependence of $\tau_{\mathrm{e}}$ on $\left(n_{\mathrm{s}}\right)^{-1 / 3}$ indicates that typical metallic spin fluctuation times $\tau_{\mathrm{c}} \simeq 10^{-15}(\mathrm{~s})$ will be reached for an average distance of the paramagnetic defects of approximately $3 \AA$. At low temperatures, in the supercooled state, the appropriate correlation time was the time of association of the electron and a particular nucleus. The observed loss of frequency dependence of $1 / T_{1}$ derives from long motional correlation times related with long polymer chains. One then obtains:

$$
\frac{1}{T_{1}}=\frac{1}{T_{2}}=\frac{C_{\mathrm{s}}}{\tau_{\mathrm{a}}}
$$

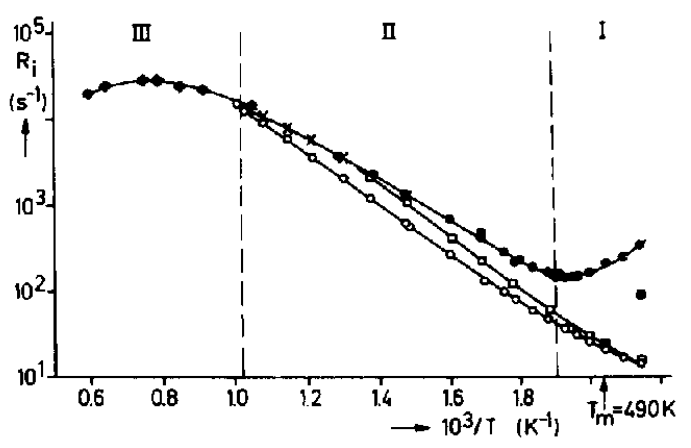

FIG. 38. Spin-lattice $\left(1 / T_{1}\right)(013.6 \mathrm{MHz}, 5 \mathrm{MHz})$, spin-spin $\left(1 / T_{2}\right)(013.6 \mathrm{MHz}, 5 \mathrm{MHz})$ and spin-phase memory $\left(1 / T^{*}\right)(40 \mathrm{MPa}, \times \mathrm{SVP})$ relaxation rates of ${ }^{77} \mathrm{Se}$ versus $1 / T$ for liquid selenium. ${ }^{(283)}$ 

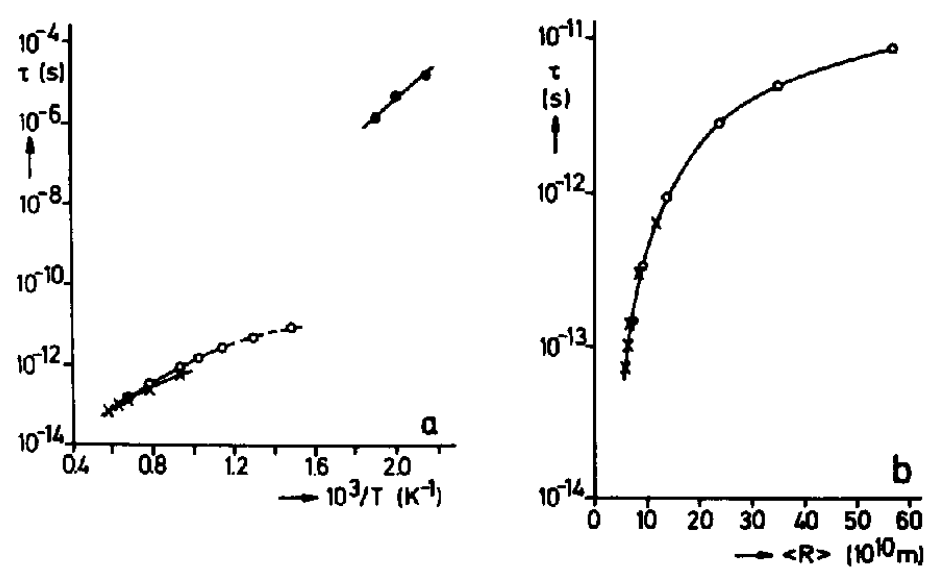

FIG. 39. (a) Molecular reorientation $\tau_{2}(0)$ and hyperfine field $\tau_{e}(010 \mathrm{MPa}, \times 40 \mathrm{MPa})$ correlation times in liquid selenium versus $1 / T$. (b) ${ }^{77} \mathrm{Se}$ hyperfine field correlation time $\tau$ versus average distance $\langle R\rangle$ of the spins at (o) $10 \mathrm{MPa}$ and $(x) 40 \mathrm{MPa} .{ }^{(283)}$

with $C_{\mathrm{s}}$ the concentration of paramagnetic centres. Extrapolation of the high temperature $\tau_{\mathrm{e}}$ results yielded $\tau_{\mathrm{e}}\left(T_{\mathrm{m}}\right) \simeq 3 \times 10^{-11}(\mathrm{~s})$, whereas the low temperature rotational correlation times $\tau_{\mathrm{r}}$ implied $10^{-9}(\mathrm{~s})<\tau_{\mathrm{a}}\left(T_{\mathrm{m}}\right)<10^{-7}(\mathrm{~s})$.

\subsection{Non-Associated Liquids}

6.2.1. Simple Non-Viscous Inorganic Liquids. The most simple molecular liquid studied with HPNMR techniques is compressed liquid nitrogen. Armstrong and Speight ${ }^{(285)}$ reported the investigation of the pressure and temperature dependence of the ${ }^{14} \mathrm{~N}$ spin-lattice relaxation rate (Fig. 40).

The $T_{1}$ measurements were used to obtain the behaviour of the activation enthalpy $\Delta H^{*}$ and activation volume $\Delta V^{*}$ for the molecular reorientations described as activated rate processes. The activation enthalpy should provide a measure of the energy required to reorient a molecule under

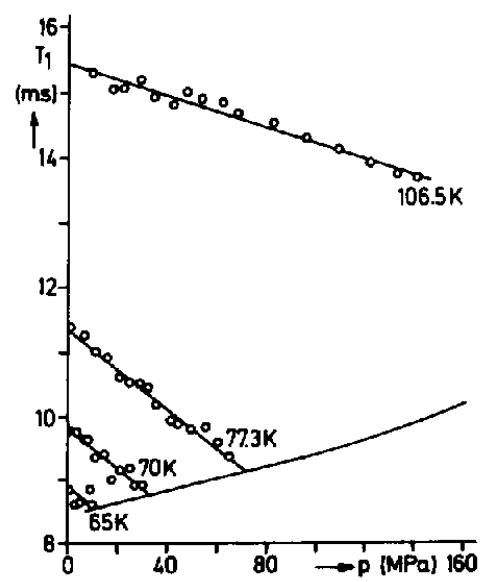

Fig. 40. Isothermal pressure dependence of the ${ }^{14} \mathrm{~N}-T_{1}$ of liquid nitrogen. The solid curve represents the melting pressure curve of nitrogen. ${ }^{(285)}$ 
conditions of constant pressure. It was observed to increase linearly with pressure, and to be independent of temperature. This is to be expected, as increased external hydrostatic pressure reduces the free volume per molecule, so that the restoring torques hindering molecular reorientations are increased. Similarly, activation volumes were found to be independent of pressure and to decrease linearly with increasing temperature. As the latter is increased, molecular jostling increases. The environment of any molecule undergoes larger fluctuations and the molecules can reorient more freely. The activation energy $\Delta E^{\#}$ for the molecular reorientations is related to $\Delta H^{*}$ and $\Delta V^{*}$ via eqn (16), and hence requires a knowledge of the thermal expansivity $\alpha$ and the isothermal compressibility $\kappa$. From $p, \rho, T$-data of liquid nitrogen the pressure and temperature dependence of $\alpha$ and $\kappa$ can be obtained and $\Delta E^{*}(T, p)$ calculated. The activation energy $\Delta E^{*}$ under constant volume conditions was found to increase both with pressure and temperature. If the volume is kept constant, then as the temperature increases, the fluctuations in the intermolecular restoring torques exerted on a molecule increase, but the average value of these torques remains constant. Armstrong and Speight concluded that their findings indicate that this behaviour results in an increased barrier to reorientation.

Though liquid halomethanes have been extensively investigated by NMR, few studies have applied hydrostatic pressure to unravel volume and energy effects upon the dynamic structure of the liquid. A continued interest in these studies further focused onto angular momentum correlation times $\tau_{\mathrm{J}}$ and their relation to reorientational correlation times $\tau_{\mathbf{L}}$ within various theories of rotational dynamics in liquids. This is because spin-rotation relaxation seems to be the only source of information about the decay of angular momentum correlations due to incessant collisions in dense fluids. As the spin-rotation interaction is effective as a relaxation mechanism only in the case of unhindered rotational motions of small molecules, a large part of the work has been performed at high temperatures in the gaseous and fluid phases. Though gas phase dynamics will generally not be considered in this review, spin-rotation relaxation at low densities will be included for those substances where high pressure NMR experiments at liquid densities have been performed.

Nuclear spin relaxation in methane and its deuterated modifications have been reported by several groups since the early days of NMR. ${ }^{(286-290)}$ These groups investigated the density and temperature dependence of the proton and deuteron spin-lattice relaxation mainly in the gas phase. In a dilute gas, spin-dependent intramolecular interactions like spin-rotation, dipolar and quadrupolar couplings fluctuate in time because collisions between molecules give rise to transitions between rotational states $|J, M\rangle$ of the molecules. These fluctuations enable the nuclear spin system to exchange energy with the rotational and translational degrees of freedom of the molecules. As a result the nuclear spin system approaches equilibrium at the 'lattice' temperature $T$. In all these studies, the relaxation has been found to be exponential with a single time constant $T_{1}$. This demonstrates that intramolecular dipole-dipole interactions do not play a dominant role. In fact, Bridges et al. ${ }^{(289)}$ showed that in the dilute gas phase, spin-lattice relaxation of the protons is almost exclusively due to spin-rotation interactions and electric quadrupole interactions dominate the relaxation of the deuterons.

The dependence of $T_{1}$ on pressure in polyatomic gases is governed by the density dependence of the various correlation times $\tau$ which are connected with the lifetime of a $|J, M\rangle$ state of the molecule. These time constants $\left(\tau_{J}, \tau_{L}\right)$ may be related to the collision frequency of a hard sphere system

$$
\frac{1}{\tau_{\text {Coll }}}=4 n \sigma^{2}\left(\frac{\pi k_{\mathrm{B}} T}{m}\right)^{1 / 2} g(\sigma)
$$

where $n$ is the number density, $\sigma$ is the effective hard sphere diameter of the molecules of mass $m$, and $g(\sigma)$ is the contact hard spheres pair distribution function. ${ }^{(138,140,291)}$ To quantify the relation between $\tau$ and $\tau_{\text {coll }}$ three approximations have been proposed in the literature based on the kinetic transport theory. Bloom and Oppenheim ${ }^{(292)}$ proposed the 'weak collision limit' appropriate to low density gases, and the 'strong collision limit' in the case of high density gases and liquids. Strong collision implies that the molecules are found with equal probability in any of their $|J, M\rangle$ states within a $J$-manifold. The correlation times are independent of $J$ and proportional to the kinetic collision time $\tau_{\text {coll }}$, hence: $:^{(293)}$

$$
\left(\tau_{\mathrm{J}}, \tau_{2}\right) \sim m^{1 / 2} T^{-1 / 2} ; R_{1, \mathrm{DD}} \sim T^{-1 / 2} ; R_{1, \mathrm{SR}} \sim T^{1 / 2},
$$


and the intramolecular dipole-dipole relaxation rate $R_{1, \mathrm{DD}}$, and the spin-rotation relaxation rate $R_{1}$ sR, show an opposite temperature dependence. The weak collision approximation, however, implies that many collisions are necessary to cause transitions between different $M$-states. The correlation times $\left(\tau_{2}, \tau_{\mathrm{J}}\right)$ are thus much longer than the mean time $\tau_{\text {coll }}$ between collisions and one obtains:

$$
\left(\tau_{\mathrm{J}}, \tau_{2}\right) \sim\left(I_{\mathrm{o}} / \mathrm{m}^{1 / 2}\right) T^{3 / 2} ; R_{1, \mathrm{DD}} \sim T^{3 / 2} ; R_{1, \mathrm{SR}} \sim T^{5 / 2},
$$

with $I_{0}$ the moment of inertia of the molecule. Johnson and Waugh ${ }^{(288)}$ assumed that the collision cross section for molecular reorientation $\Phi$ is proportional to the inverse square of the relative speed $v$ of a pair of colliding molecules. In this 'transient approximation' one obtains:

$$
\left(\tau_{\mathrm{J}}, \tau_{2}\right) \sim m^{-1 / 2} T^{1 / 2} ; R_{1, \mathrm{DD}} \sim T^{1 / 2}, R_{1, \mathrm{SR}} \sim T^{3 / 2} .
$$

Within the binary collision approximation the correlation frequencies are always proportional to the number density $n$. Hence in low density systems one finds:

$$
\left(\tau_{\mathrm{J}}, \tau_{2}\right) \sim[n\langle\boldsymbol{\Phi} \mathbf{\Phi}\rangle]^{-1} .
$$

This density dependence implies that:

and

$$
T_{1} \sim n \text { for } \omega_{\mathrm{L}} \tau \ll 1
$$

$$
T_{1} \sim n^{-1} \text { for } \omega_{\mathrm{L}} \tau \gg 1,
$$

in accordance with the assumed Lorentzian spectral densities (eqn 38). There must then exist a characteristic density for which $T_{1}$ is a minimum. (294-296)

The temperature dependence of the proton relaxation rate $R_{1}\left({ }^{1} \mathrm{H}\right)$ of methane and its deuterated modifications $\mathrm{CH}_{4-x} \mathrm{D}_{x}$ yields:

$$
\rho R_{1, \mathrm{SR}} \sim T^{3 / 2},
$$

and hence a square root temperature dependence of the angular momentum correlation time $\tau_{\mathrm{J}} \sim T^{1 / 2}$. The latter corresponds to the transient approximation, though the collisions are not weak. Rather $\tau_{\mathrm{J}} \simeq \tau_{\text {Coll }}$ has been found ${ }^{(289)}$ in accord with an intermediate coupling which is neither in the weak $\left(\tau_{\mathrm{J}} \gg \tau_{\text {Coll }}\right)$ nor in the strong $\left(\tau_{\mathrm{J}} \ll \tau_{\text {Coll }}\right)$ collision limit.

The differences in the proton relaxation times of the partially deuterated methanes were found to be small as would be expected in the case of a dominant spin-rotation relaxation mechanism. Isotope effects are much larger, however, in the case of the corresponding deuterium relaxation times. They can be explained in terms of purely geometrical effects on the corresponding free rotor correlation function due to different orientations of the principal coordinate system of the electric field gradient-tensor relative to the molecule-fixed frame. The reason for these large geometry effects can be traced back to the fact that in low density gases the separations of the rotational levels are much larger than their widths. This is no longer true in the dense fluid and liquid phases where the reorientational correlation frequency $\left(1 / \tau_{2}\right)$ is greater than the average splitting between neighbouring states of different $M$ within a $J$-manifold. Consequently the values of $T_{1}$ for the deuterons in liquid and solid $\mathrm{CD}_{4}$ and $\mathrm{CHD}_{3}$ are almost identical as well as being almost independent of temperature. ${ }^{(297)}$

By far the most extensive NMR study of methane has been reported by the group of Trappeniers and collaborators. ${ }^{(293,298-300)}$ They measured the proton spin-lattice relaxation time $T_{1}\left({ }^{1} \mathrm{H}\right)$ as well as the self-diffusion coefficients of $\mathrm{CH}_{4}$ and its partially deuterated analogs, and varied the temperature and the density over a wide range covering the gaseous, liquid and solid phases (Fig. 41). Furthermore, $\mathrm{CH}_{4}-\mathrm{CD}_{4}$ mixtures of different compositions have been studied over the same $p, T$-range.

Contrary to previous investigations ${ }^{(301,302)}$ these very precise measurements allowed a separation of the various contributions to the total proton relaxation rate:

$$
R_{1}\left({ }^{1} \mathrm{H}\right)=R_{1, \mathrm{DD}}^{\text {intra }}+R_{1, \mathrm{DD}}^{\mathrm{inter}}+R_{1, \mathrm{sR}} \text {. }
$$

In accordance with earlier findings discussed above, intermolecular dipolar couplings can be neg- 


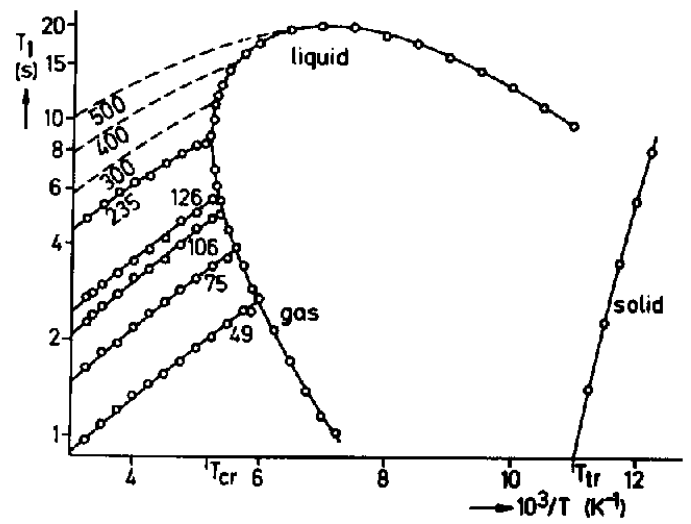

FIG. 41. Proton spin-lattice relaxation time $T_{1}$ of methane $\left(\mathrm{CH}_{4}\right)$ along the sublimation line, in the coexistence region and along the isochores. Densities are given in units of the density at standard conditions $\rho / \rho_{\text {stp }}$. Broken lines are obtained from interpolation of the isotherms. ${ }^{(298)}$

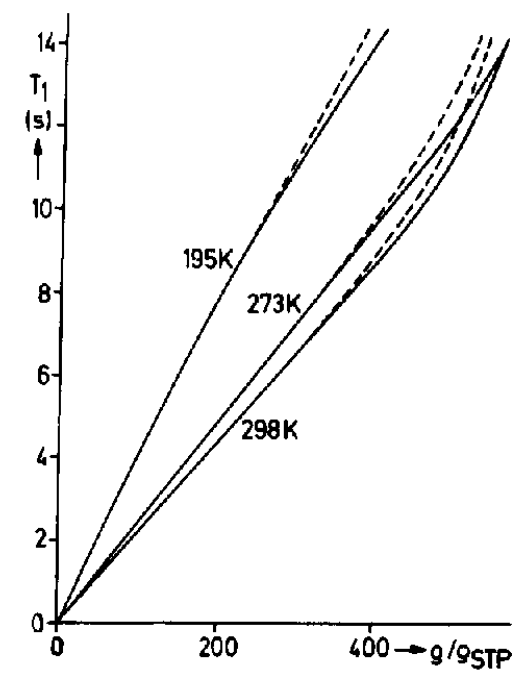

Fig. 42. Experimental proton relaxation time $T_{1}(-)$ and corresponding intramolecular contribution ( - ) due to dipole-dipole and spin-rotation relaxation versus reduced density in $\mathrm{CH}_{4}{ }^{(293)}$

lected at low densities as shown in Fig. 42. In addition, intramolecular dipolar contributions amount to $10 \%$ of the total rate at most. Hence the spin-rotation coupling provides the dominant relaxation mechanism in methane at low densities.

Up to rather large densities $\left(\rho / \rho_{\mathrm{STP}}<250\right)$ a linear density dependence of the proton relaxation time $T_{1}$ has been found in good agreement with predictions from kinetic theory. The angular momentum correlation times $\tau_{\mathrm{J}}$ at these densities are of the same order of magnitude as the kinetic collision time $\tau_{\text {coll }}$, excluding the weak collision limit and favouring the transient approximation for gaseous methane.

At liquid densities, the spin-rotation relaxation still dominates at high temperatures close to the critical point along the liquid-vapour coexistence line. At lower temperatures, close to the triple point, the intermolecular dipole-dipole relaxation is dominant, with the intramolecular dipolar contribution 
being almost negligible over the whole liquid range. The evaluation of the relaxation rates within the diffusion model ${ }^{(104.303)}$ leads to several inconsistencies, not the least being the failure of the Hubbard relation, eqn (71) to fit the data. The calculation of $R_{1, \text { intra }}$ and $R_{1, \mathrm{SR}}$ within a kinetic theory in the transient approximation leads to results which are consistent with experiment even at high densities where higher-order collisions become increasingly important.

Fluoromethanes (methylfluoride $\mathrm{CH}_{3} \mathrm{~F}$, fluoroform $\mathrm{CHF}_{3}$, carbon tetrafluoride $\mathrm{CF}_{4}$ ) have also been investigated by HP-NMR. Most studies, however, concerned the spin-lattice relaxation in gaseous samples. ${ }^{(304-309)}$ Armstrong and Courtney ${ }^{(307)}$ reported spin-lattice relaxation times $T_{1}$ of ${ }^{1} \mathrm{H}$ and ${ }^{19} \mathrm{~F}$ nuclei measured on gaseous samples of $\mathrm{CH}_{3} \mathrm{~F}$ and $\mathrm{CHF}_{3}$ at room temperature for densities $0.03 \leqslant \rho / \rho_{\text {STP }} \leqslant 10$ Amagat in the important regime of the $T_{1}$ minimum. In each case the spin-lattice relaxation was dominated by the spin-rotation interaction. These data provided a stringent test of the theory of the spin-rotation relaxation mechanism in spherical top and symmetric top molecules including the role of nuclear spin symmetry as well as the theory of rotational dynamics in low density fluids. Using kinetic theory $\left(\tau \sim \rho^{-1}\right)$ both proton $\left({ }^{1} \mathrm{H}\right)$ and fluorine $\left({ }^{19} \mathrm{~F}\right)$ relaxation times $T_{1, \text { sR }}$ can be expressed as: ${ }^{(307)}$

$$
\left(T_{1} / \rho\right)=a+b / \rho^{2},
$$

with $a$ and $b$ being proportional to the inverse square of the effective spin-rotation coupling strength. The latter may be compared with results from molecular beam experiments in the gas phase. Considerable differences have been found in the case of methylfluoride, whereas agreement was obtained for fluoroform. Some of these discrepancies are certainly due to the single correlation time approximation. The relaxation times show a linear density dependence at intermediate densities $\left(1 \leqslant \rho / \rho_{\text {STP }} \leqslant 20\right)$ and in some cases also a $T_{1}$ minimum at low densities as is shown in Fig. 43. Large deviations from linearity were, however, observed in $T_{1}\left({ }^{1} \mathrm{H}\right)$ of both methylfluoride and fluoroform at densities $\rho / \rho_{\mathrm{STP}}>20$ whereas the corresponding curves for methane are linear up to much higher densities $\rho / \rho_{\text {STP }} \simeq 250$. Hence higher-order collisions seem to be much more important in the symmetric top molecules $\mathrm{CH}_{3} \mathrm{~F}$ and $\mathrm{CHF}_{3}$ compared to the spherical top molecule $\mathrm{CH}_{4}$.

In the liquid phase, relaxation rate measurements under ambient pressure were reported for fluoroform by Johnson et al., ${ }^{(309)}$ Chaffin and Hubbard, ${ }^{(310)}$ and Harrell ${ }^{(311)}$ and in the solid phase by
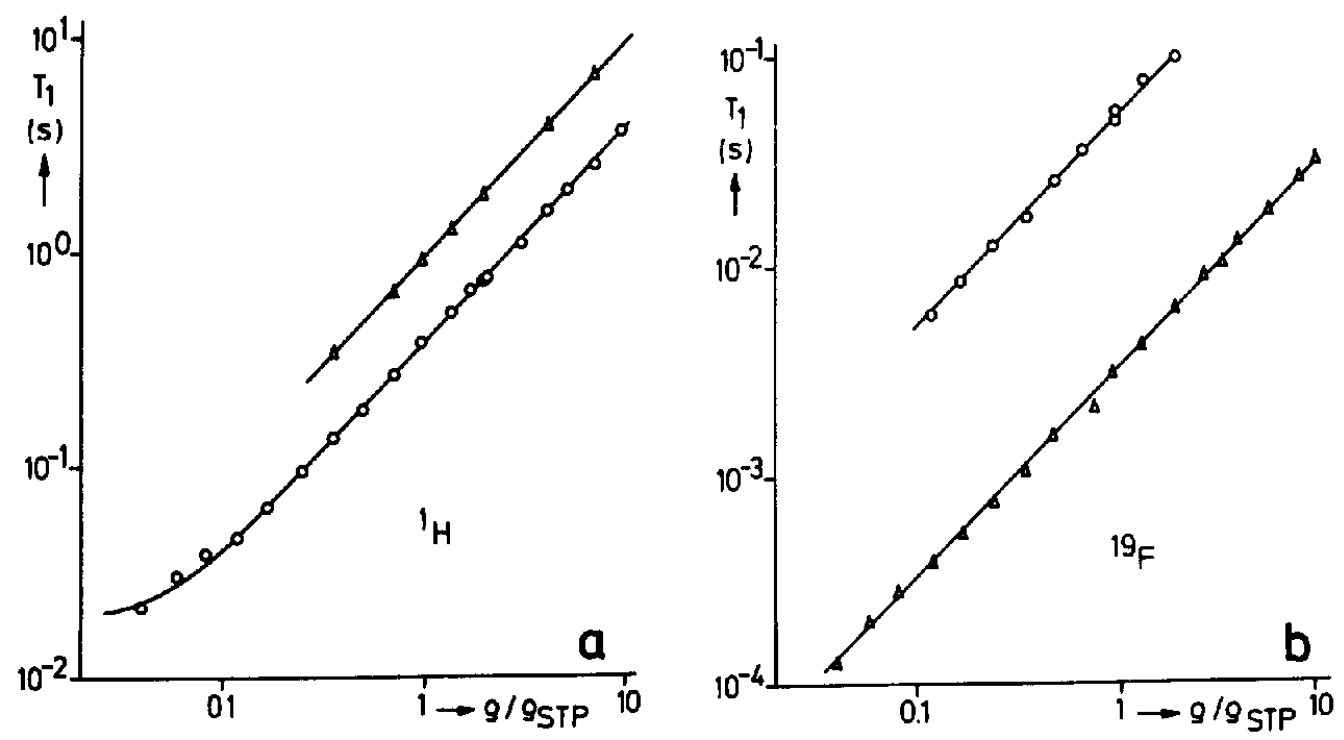

Fig. 43. (a) Proton $\left({ }^{1} \mathrm{H}\right)$ and (b) fluorine-19 $\left({ }^{19} \mathrm{~F}\right)$ relaxation times $T_{1}$ in methylfuoride $(\mathrm{o})$ and fluoroform $(\Delta)$ versus reduced density. ${ }^{(307)}$ 
Watton et $a l^{(312)}$ The only high pressure liquid state NMR experiments have been performed by Lang et al. ${ }^{(313.314)}$ in methylfluoride $\left(\mathrm{CH}_{3} \mathrm{~F}\right)$, and also in fluoroform $\left(\mathrm{CHF}_{3}\right)$. They measured the proton $\left({ }^{1} \mathrm{H}\right)$-, deuterium $\left({ }^{2} \mathrm{H}\right)$ - and fluorine $\left({ }^{19} \mathrm{~F}\right)$ relaxation rates $R_{1}$ as well as self-diffusion coefficients $D_{\mathrm{H}}$ over an extended range of temperatures $(150 \mathrm{~K} \leqslant T \leqslant 450 \mathrm{~K})$ and pressures $p \leqslant 250 \mathrm{MPa}$. Whereas high frequency experimental techniques like IR and Raman bandshape investigations, light scattering techniques and dielectric measurements yield orientational correlation functions $C_{\mathrm{L}}(t)$ over a limited time range, nuclear magnetic relaxation experiments have limitations in probing details of the microdynamics of orientational and positional fluctuations. This is because in low viscosity liquids only the area of the relevant time correlation function is normally determined and not its shape, yielding average orientational $\left(\tau_{\mathrm{L}=2}\right)$ and/or translational $\left(\tau_{\mathrm{d}}\right)$ correlation times. However, in low molecular weight liquids, spin-rotation interactions often dominate the relaxation of spin $1 / 2$ nuclei $\left({ }^{1} \mathrm{H},{ }^{19} \mathrm{~F}\right)$ providing angular momentum (velocity) correlation times $\tau_{\jmath}\left(\tau_{\omega}\right)$. The importance of simultaneous measurements of $\tau_{2}$ and $\tau_{\omega}$ lies in the model dependent relationship between these microscopic parameters. Thus together they may provide a deeper insight into the microdynamics of rotational motions in these liquids.

A clean source of information about orientational correlation times $\tau_{2}$ is provided by deuterium spin-lattice relaxation rates $R_{1}\left({ }^{2} \mathrm{H}\right)$ if independent information from solid state NMR or gas phase molecular beam studies is available on the magnitude of the deuterium quadrupole coupling constant (QCC). The latter usually does not show any significant state dependence in these simple liquids. ${ }^{\text {(31 5) }}$ In symmetric top molecules like $\mathrm{CD}_{3} \mathrm{~F}$ and $\mathrm{CDF}_{3}$ the integral correlation time $\tau_{2}$ is actually a composite of two motional modes: a usually fast spinning motion around the symmetry axis and a slower tumbling motion of this axis. Hence two quadrupolar nuclei in non-equivalent positions within the molecule are necessary to extract the corresponding correlation times $\tau_{2 \perp}$ and $\tau_{2 \|}$ in methylfluoride. These are not available, of course. Intramolecular dipole-dipole couplings would sense these modes also, but their contribution to the total proton relaxation rate is almost negligible and cannot be determined reliably. Within a diffusion model, which is not appropriate at all as inertial effects must be important in the case of the fast spinning motion, the integral correlation time can be related to the components of the diffusion tensor $D$ via

$$
\tau_{2}=\frac{(1 / 2)\left(3 \cos ^{2} \theta-1\right)^{2}}{6 D_{\perp}}+\frac{3 \sin ^{2} \theta \cos ^{2} \theta}{5 D_{\perp}+D_{\|}}+\frac{(3 / 4) \sin ^{4} \theta}{2 D_{\perp}+4 D_{\|}},
$$

with $\theta$ the angle between the molecular symmetry axis and the $C-D$ bond axis. As a rule, a large inertial anisotropy $I_{\|} / I_{\perp}$ corresponds to a substantial diffusional anisotropy $D_{\|} / D_{\perp}$. Thus within the series of halomethanes, methylfiuoride was expected to exhibit the smallest motional anisotropy. This was corroborated by dielectric relaxation measurements ${ }^{(316)}$ which gave $D_{\perp}=\left(2 \tau_{1}\right)^{-1}$ along the coexistence line yielding a modest motional anisotropy $\xi=D_{\|} / D_{1} \simeq 2$ for $\mathrm{CD}_{3} \mathrm{~F}$ only. Hence integral orientational correlation times $\tau_{2}$ have been discussed in the case of $\mathrm{CD}_{3} \mathrm{~F}$ at elevated pressures. In fluoroform, electric quadrupole interactions of the deuterium nucleus are modulated by the tumbling motion of the symmetry axis only, as the latter coincides with the $\mathrm{C}-\mathrm{D}$ bond axis. However, as with methylfluoride, molecular reorientations have also been found to be nearly isotropic in fluoroform. ${ }^{(311)}$

A direct comparison of the density dependence of the self-diffusion coefficient $D_{\mathrm{H}}$ in $\mathrm{CH}_{3} \mathrm{~F}$ and $\mathrm{CHF}_{3}$ with the corresponding integral orientational correlation times $\tau_{2}$ (Figs 44 and 45) revealed that orientational fluctuations are less hindered by compression or removal of thermal energy than are positional fluctuations. Further, the density dependence of rotational motions $\left(\tau_{2}\right)$ is only modest at high temperatures and strongest at low temperatures, whereas the contrary is true for translational motions $\left(D_{\mathrm{H}}\right)$. Hence it must be concluded that rotation-translation coupling cannot be strong in both liquids. At constant temperature the correlation times increased in a non-linear fashion with density, reflecting the retarding action of molecular torques due to anisotropic intermolecular interactions upon the reorientation process at higher packing fractions. Nevertheless, integral correlation times turned out to be rather short $\left(\tau_{2}<1 \mathrm{ps}\right)$ in methylfuoride and only slightly longer in fluoroform. Together with the low barrier to rotation, $E_{\rho}^{\#}=2.48(\mathrm{~kJ} / \mathrm{mol})$ in $\mathrm{CD}_{3} \mathrm{~F}$ and $E_{\rho}^{\#}=2.75$ $(\mathrm{kJ} / \mathrm{mol})$ in $\mathrm{CDF}_{3}$, this suggests the importance of inertial effects during the reorientation process 

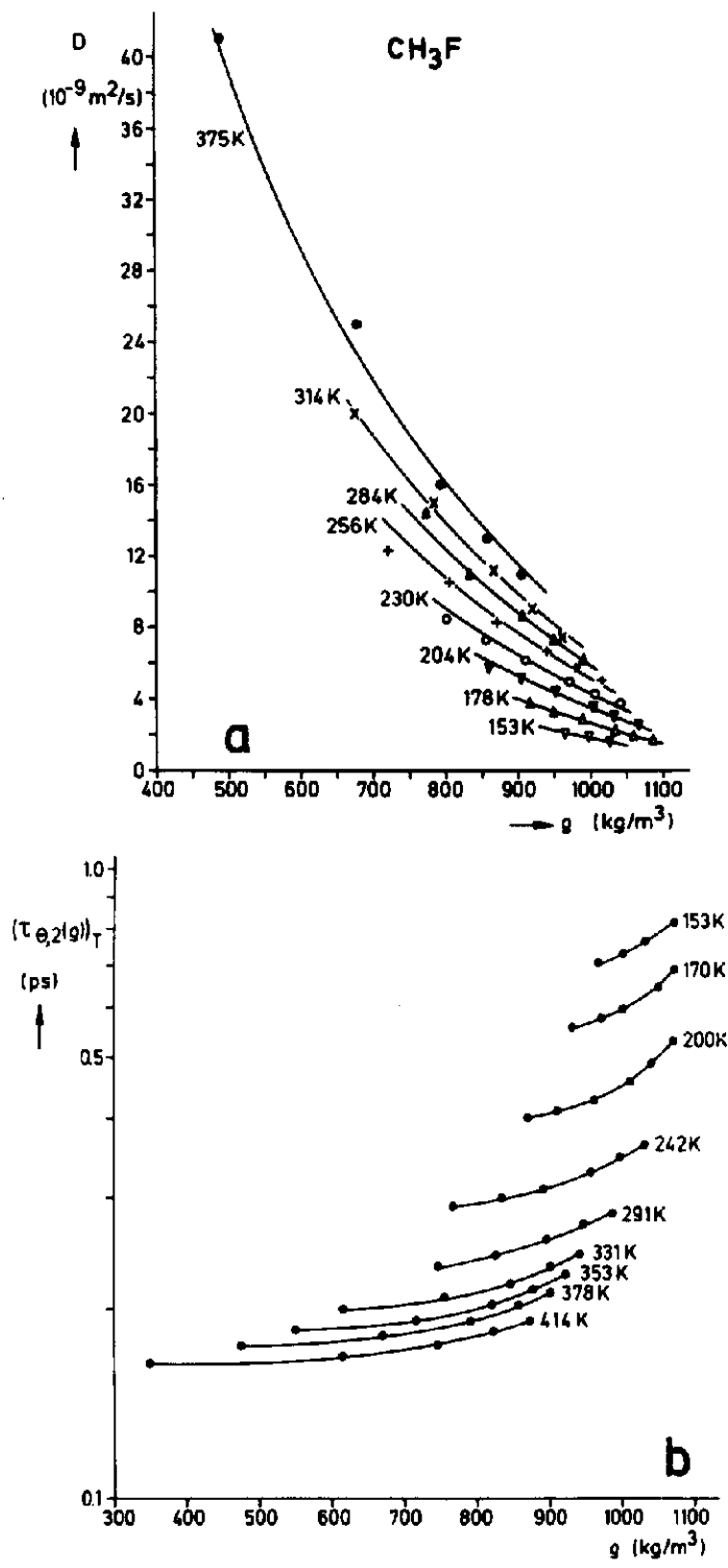

FIG. 44. Isothermal density dependence of (a) the self-diffusion coefficient $D$ and (b) the orientational correlation time $\tau_{2}$ of methylfluoride $\left(\mathrm{CH}_{3} \mathrm{~F}\right)^{(313)}$

which must proceed rather unhindered. An additional indication that the rotational dynamics of methylfluoride and fluoroform do not correspond to a small step diffusion process over most of the $p, T$-range investigated was obtained by a comparison of the integral correlation times $\tau_{2}$ with the free rotor correlation time $\tau_{\mathrm{f}}=(3 / 5)(\langle I\rangle / k T)^{1 / 2}$. If $\tau_{2} / \tau_{\mathrm{f}} \gg 1$, rotational motions are certainly diffusive and inertial effects may be neglected. But if $\tau_{2} / \tau_{f} \leqslant 1$ the rotational propagator $P\left(\Omega_{0}, \Omega, \tau\right)$ does not follow a diffusion equation ${ }^{(303,317)}$ and inertial effects must be included. ${ }^{(86,318-320)}$ 

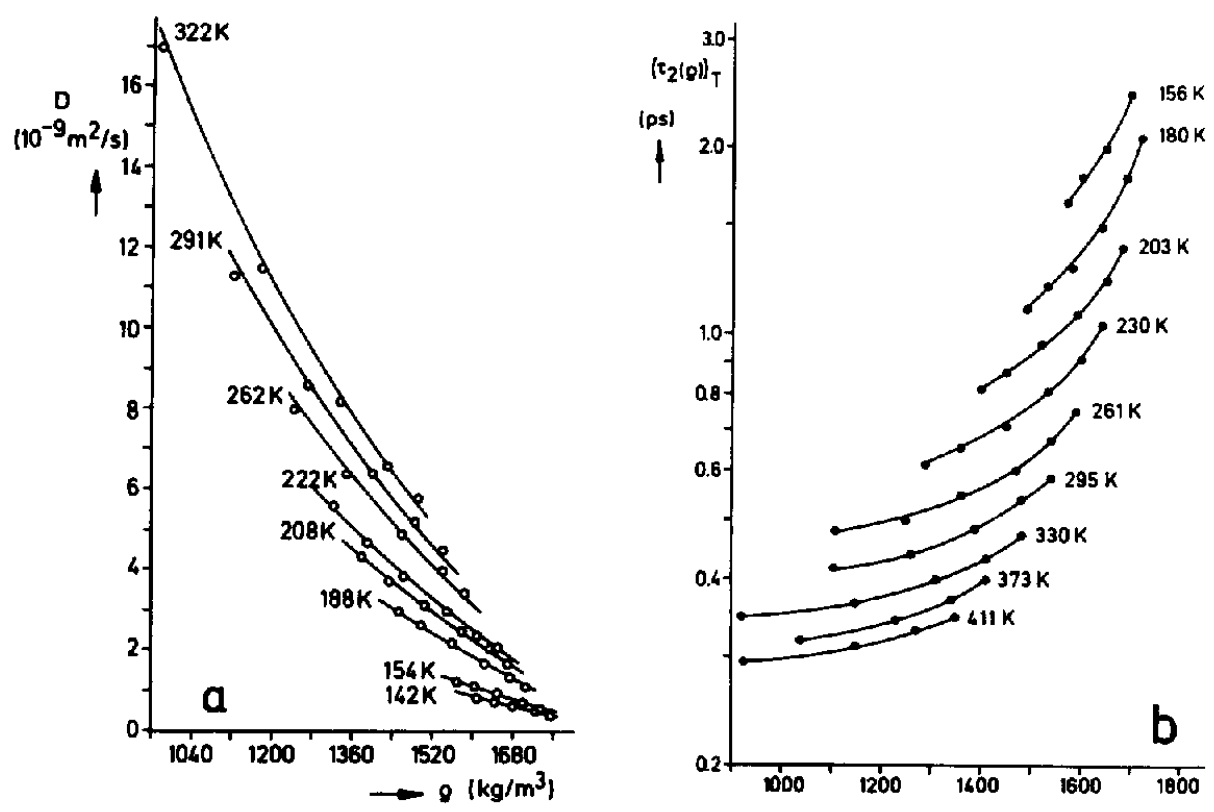

Fig. 45. Isothermal density dependence of (a) the self-diffusion coefficient $D$ and (b) the orientational correlation time $\tau_{2}$ of fluoroform $\left(\mathrm{CHF}_{3}\right)^{(314)}$

The spin-lattice relaxation of the protons and fluorine nuclei proceeds mainly via magnetic dipole-dipole and spin-rotation interactions. The proton relaxation in methylfluoride and fluoroform is dominated over most of the $p, T$-range investigated by intermolecular dipole couplings modulated by translational motions, contrary to the gas phase as discussed above. The corresponding relaxation rates $R_{1, \text { inter }}\left({ }^{1} \mathrm{H}\right)$ could be evaluated with eqns $(48-51)$ where use was made of the measured self-diffusion coefficient $D$ of $\mathrm{CH}_{3} \mathrm{~F}$ and $\mathrm{CHF}_{3}$ respectively, and the distance of closest approach was taken from MD simulations. ${ }^{(321)}$ The relaxation of the ${ }^{19} \mathrm{~F}$-nuclei is dominated by spin-rotation interactions even in the liquid phase. This predominance is especially pronounced in the deuterated compounds, and is much stronger in $\mathrm{CD}_{3} \mathrm{~F}$ than $\mathrm{CDF}_{3}$ because of its smaller molecular weight. A self-consistent treatment of all the ${ }^{2} \mathrm{H}-,{ }^{1} \mathrm{H}$ - and ${ }^{19} \mathrm{~F} T_{1}$ data allowed a reliable determination of the spin-rotation relaxation rates $R_{1, \mathrm{SR}}\left({ }^{19} \mathrm{~F}\right)$ in methylfuoride and fluoroform. With the components of the spin-rotation interaction tensor $C$ deduced from molecular beam and NMR relaxation experiments in the gas phase, these relaxation rates allowed the determination of spin-rotation correlation times $\tau_{\text {sk }}$ (eqn (68)), which are closely related to angular velocity correlation times $\tau_{w p}$. The density dependence of the reduced spin-rotation correlation times $\tau_{\mathrm{SR}}^{*}=\tau_{\mathrm{SR}}(k T /\langle I\rangle)^{1 / 2}$ is compared in Fig. 46 for methylfluoride and fluoroform. With increasing density the correlation times $\tau_{\mathrm{SR}}^{*}$, hence $\tau_{\omega}$ or $\tau_{\mathrm{J}}$, decrease in a non-linear fashion, with a somewhat stronger dependence on density observed for fluoroform compared to methylfluoride. The isochoric temperature dependence of $\tau_{\mathrm{SR}}^{*}$ exhibited Arrhenius type behaviour for fluoroform, but was found to be non-linear for methylfluoride. It is also interesting to note that $\tau_{\mathrm{SR}}^{*}$ does not fall below a value of 0.1 , which may be regarded as an upper limit of validity of the rotational diffusion model in $\mathrm{CH}_{3} \mathrm{~F}$, whereas $\mathrm{CHF}_{3}$ seems to conform to this limit at low temperatures and high densities.

Hence molecular torques retarding rotational motions seem to be much larger in fluoroform than in methylfluoride at comparable packing fractions. In fact, the orientational correlation functions show features characteristic of high torque liquids ${ }^{(322,323)}$ with torsional oscillations at short times, which is typical for motions in a cage, and an exponential long-time tail, signifying Markovian behaviour as a simple consequence of the long range isotropy of the liquid. Integral correlation times 

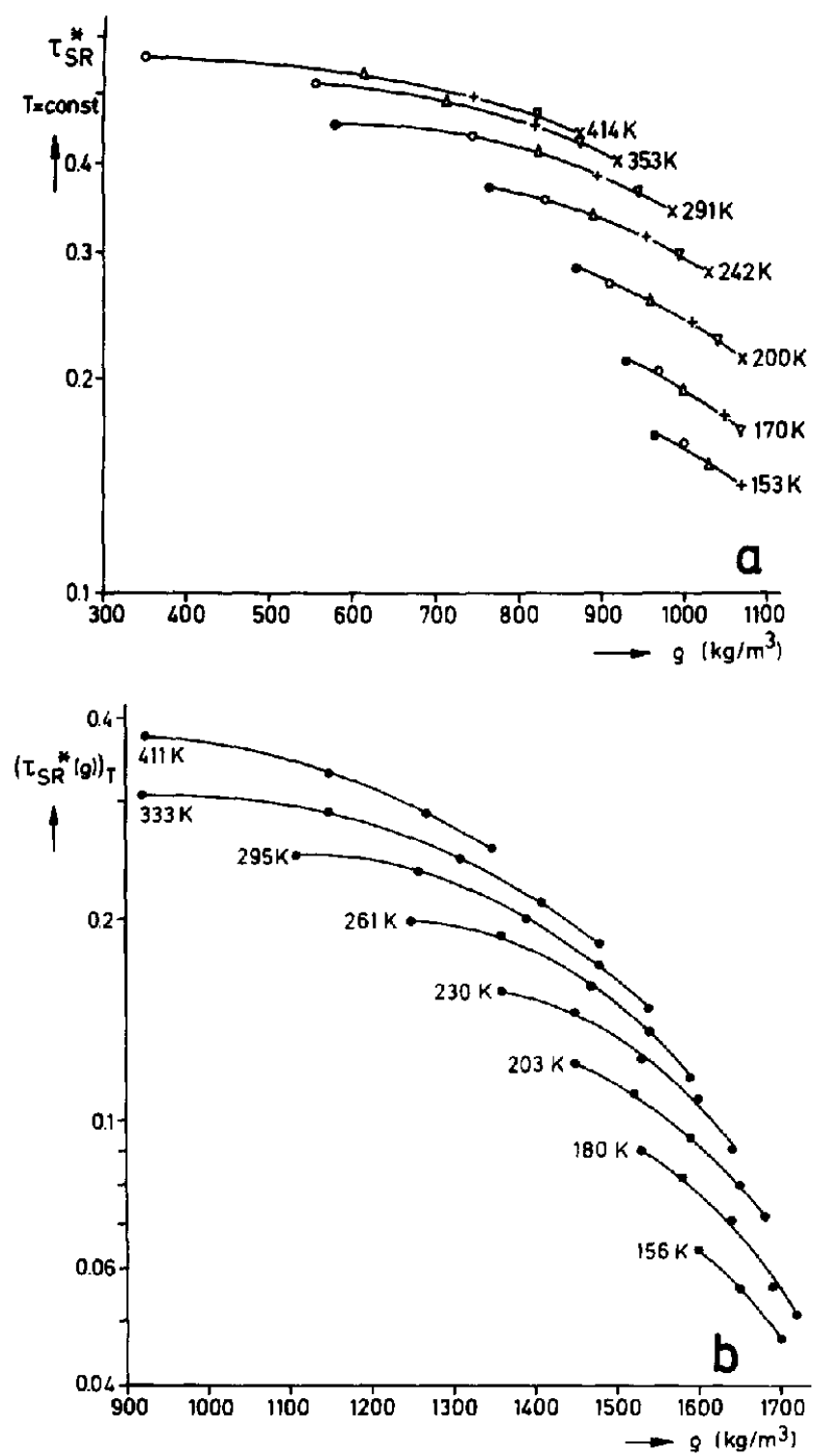

FIG. 46. Isothermal density dependence of the reduced spin-rotation correlation time $\tau_{S_{\tau}^{*}}^{*}$ in (a) methylfluoride and (b) fluoroform. ${ }^{(313,314)}$

obviously must contain these features in a nonspecific way but are mostly dominated by the Markovian nature of orientational fluctuations. Spin-rotation relaxation is related to fluctuations of both the angular velocity and the orientation of the molecules, and provides spin-rotation correlation times $\tau_{\mathrm{SR}}$ of the angular velocity-orientational product correlation function. It is only within certain models of rotational molecular dynamics that these bivariate correlation functions, and the corresponding integral correlation times, can be related to the more fundamental angular velocity (momentum) correlation times $\tau_{\omega}\left(\tau_{1}\right)$. Because reduced orientational correlation times $\tau_{2}$ may also be related to $\tau_{\omega}$ within these models, a comparison of $\tau_{2}$ and $\tau_{\text {SR }}$, determined over wide ranges of density and temperature, may provide insights into the state of molecular motions in these liquids, which is 
not easily obtainable otherwise. The two most often used motional models for this purpose are the extended diffusion (EDJ, EDM) models ${ }^{(318,319,324-328)}$ and the Fokker-Planck-Langevin (FPL) model..$^{(320,329-333)}$ When applied to spherical tops, a single parameter $\tau_{\omega}$ is changed in both models to produce the limits of free rotation and rotational diffusion. All the models assume successsive uncorrelated instantaneous collisions which cause a small change of the angular momentum in the FPL model. In the EDJ model, collisions are strong with large angular impulses which randomize the angular momentum at each collision, whereas in the EDM model only the direction of the angular momentum is randomized. But random, uncorrelated collisions cannot cause a reversal of the angular momentum as is observed in most liquids and has also been observed in methylfuoride ${ }^{(32)}$ and fluoroform. ${ }^{(322,334,335)}$

Because of these shortcomings the application of the models to high-torque liquids must be considered with some caution. Nevertheless these models have been applied to both methylfluoride and fluoroform. As the Hubbard relation $\tau_{2}^{*} \tau_{\mathrm{SR}}^{*}=1 / 6$ is not fulfilled in liquid $\mathrm{CH}_{3} \mathrm{~F}$, even in the limit $\tau_{\omega}^{*} \ll \tau_{2}^{*}$, it was concluded that the EDM model most closely represents the relation between $\tau_{2}$ and $\tau_{\mathrm{SR}}$ within the $p, T$-range studied. As the EDM model seems somewhat unphysical this result may point towards an inadequacy in the coupling strengths used to deduce the correlation times from the corresponding relaxation rates. The results in fluoroform could be well reproduced with the FPL model with the Hubbard limit being obeyed at low temperatures. In general, rotational motions in $\mathrm{CHF}_{3}$ have been found to deviate less from the rotational diffusion limit than do these motions in $\mathrm{CH}_{3} \mathrm{~F}$ when compared in the same range of number densities $n$ and reduced temperatures $T / T_{\mathrm{c}} \cdot{ }^{(313,314)}$ Finally, the experimental $\tau_{\mathrm{SR}}^{*}$ of liquid $\mathrm{CH}_{3} \mathrm{~F}$ and $\mathrm{CHF}_{3}$ have been compared to the reduced angular velocity correlation times $\tau_{\omega}^{*}$ (to which $\tau_{\mathrm{SR}}^{*}$ reduce in the limit $\tau_{\omega \omega}^{*} \ll 1$ ) as calculated within the rough hard spheres model of rotational relaxation (see eqn 72 ). ${ }^{(138-140)}$ The resulting $\tau_{\omega}^{*}$ are a factor of 2-5 larger than the value of $\tau_{\mathrm{SR}}^{*}$ deduced from the experimental relaxation rates in both methylfluoride and fluoroform.

A closely related HP-NMR study of liquid chlorodifluoromethane $\left(\mathrm{CF}_{2} \mathrm{HCl}\right)$ has been reported by Vardag and Lüdemann. ${ }^{(229)}$ Self-diffusion coefficients $D$ were measured in a temperature range $145 \mathrm{~K} \leqslant T \leqslant 400 \mathrm{~K}$ and at pressures $p \leqslant 200 \mathrm{MPa}$. The measured density and temperature dependence of $D$ is well described by the modified hard-sphere model (see Table 5) as discussed in Section 4.2.3. In addition, spin-lattice relaxation rates $R_{1}$ of the nuclei ${ }^{1} \mathrm{H},{ }^{2} \mathrm{D}$ and ${ }^{19} \mathrm{~F}$ were investigated in the same $p, T$-range and separated into their respective quadrupolar, dipolar and spin-rotation contributions. Integral orientational correlation times $\tau_{2}$ could be deduced from deuterium relaxation rates (Fig. 47a) assuming a deuterium QCC of $\chi=165 \pm 15 \mathrm{kHz}$. At constant density the correlation times $\tau_{2}$ reflect the sole influence of the kinetic energy upon the rotational motions of the molecules. An Arrhenius temperature dependence with density independent slope was found with shorter correlation times at higher temperatures and lower densities. At constant temperature the correlation times $\tau_{2}$ increase with density in a non-linear fashion.

The increase is more pronounced at low temperatures due to reduced thermal excitations of rotational states within the transient potential wells. At high densities intermolecular torques hinder orientational fluctuations strongly leading to small step diffusive reorientational processes and to increasing correlation times. The isobaric temperature dependence of the ${ }^{19} \mathrm{~F}-T_{1}$ (Fig. 47b) has pronounced maxima, with dominance of spin-rotation interactions at high temperatures and low densities, and dipolar interactions prevailing at low temperatures and high pressures. The dipolar contributions to the ${ }^{19} \mathrm{~F}$ relaxation rate could be estimated using the integral correlation times $\tau_{2}$ deduced from the ${ }^{2} \mathrm{H}-T_{1}$ data and the measured self-diffusion coefficients $D$ in eqn (45) and eqn (48). Subtracting the dipolar relaxation contribution from the total relaxation rate $R_{1}\left({ }^{19} F\right)$, the spin-rotation contribution $R_{1}$ sR could be obtained. Using eqn (68) and components $C_{\perp}=8627 \mathrm{~Hz}$ and $C_{\|\|}=492 \mathrm{~Hz}$, as deduced from absolute chemical shielding data, spin-rotation correlation times $\tau_{\mathrm{SR}}$ have been calculated. At constant temperature $\tau_{\mathrm{SR}}$ decreases with increasing density reflecting the retarding influence of intermolecular torques upon molecular reorientations at high densities and low temperatures. Mapping the reduced orientational correlation times $\tau_{2}^{*}$ versus the reduced spin-rotation correlation times $\tau_{\mathrm{SR}}^{*}$ (Fig. 48) the authors could show that the rotational dynamics of $\mathrm{CF}_{2} \mathrm{HCl}$ in the liquid state is well represented by the FPL-model. 


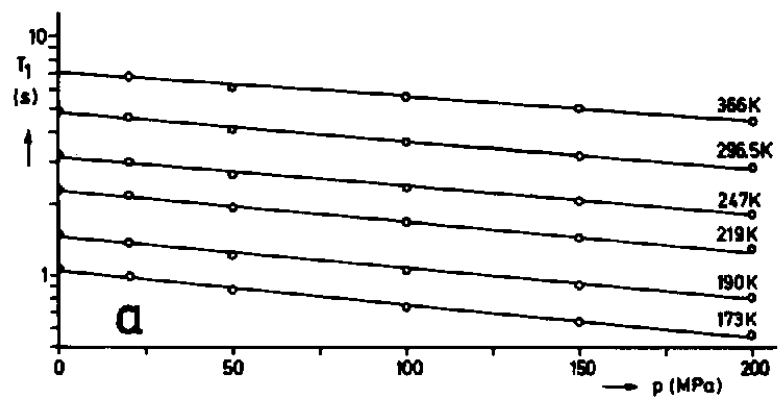

FIG. 47a. Isothermal pressure dependence of the deuteron spin-lattice relaxation time $T_{1}$ of $\mathrm{CF}_{2} \mathrm{DCl}^{(229)}$

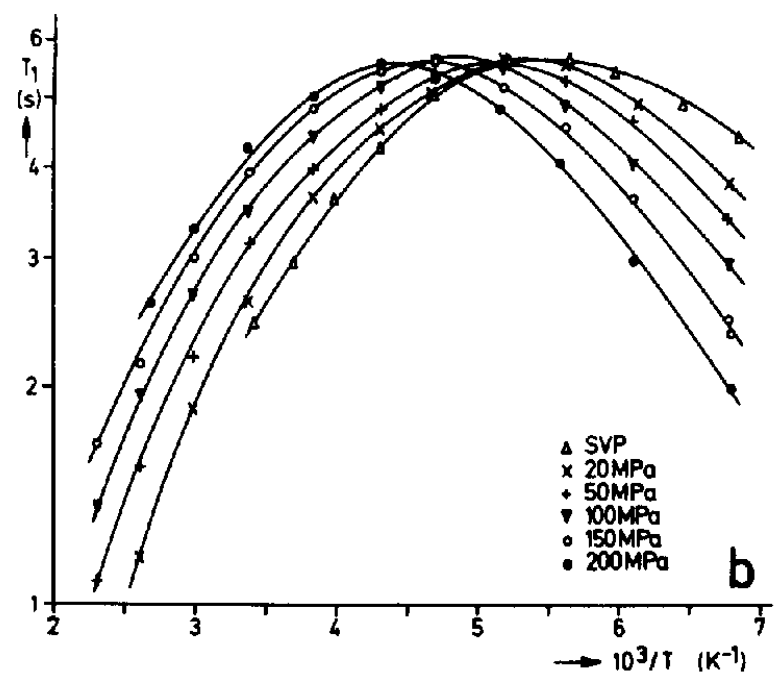

FIG. 47b. Isobaric temperature dependence of the fluorine-19 spin-lattice relaxation time $T_{1}$ in $\mathrm{CF}_{2} \mathrm{HCl}^{(229)}$

Carbon tetrafluoride $\mathrm{CF}_{4}$ is another halomethane that has been investigated thoroughly by HP-NMR in both gaseous $^{(306,336-340)}$ and dense fluid ${ }^{(341,342)}$ phases. Nuclear spin relaxation experiments on the liquid state have been reported by Rugheimer and Hubbard, ${ }^{(302)}$ though at ambient pressure only. In the gas phase the spin-lattice relaxation of the ${ }^{19} \mathrm{~F}$ nuclei has been studied as a function of density at very low densities and room temperature. The main conclusion is that the spin-rotation interaction provides the dominant relaxation mechanism. In the dilute gas phase a $T_{1}$-minimum predicted by kinetic theory (eqns $(99)$ and $(100)$ ) was observed. This minimum allows an estimate of reorientational cross-sections $\phi$ from the minimum condition $(\omega \tau=1)$ and the appropriate expression for the effective correlation time (within a single correlation time approximation) taken from kinetic theory:

$$
\omega_{\mathrm{L}} \tau=1=\omega_{\mathrm{L}}\left(n_{\min } N_{\mathrm{L}} v \phi\right),
$$

with $\nu=(8 \mathrm{kT} / \pi \mu)^{1 / 2}$ being the mean relative velocity of a colliding pair of molecules of reduced mass $\mu$, and $N_{L}$ is Avogadro's constant. The ratio of the effective rotational cross-section $\phi$ to the geometric cross-section $\phi_{\mathrm{g}}(=\pi)$ gives the number of collisions necessary to randomize the angular momentum of the molecules. This number turns out to be close to unity at these densities. Furthermore, effective spin-rotation interaction constants, $C_{\text {eff }}^{2}=C_{0}^{2}+(4 / 45) \Delta C^{2}$, could be obtained from fitting the density dependence of $T_{1}$ to eqns (38), (68) and (94) and from a knowledge of the density $n_{\min }$. Together with 


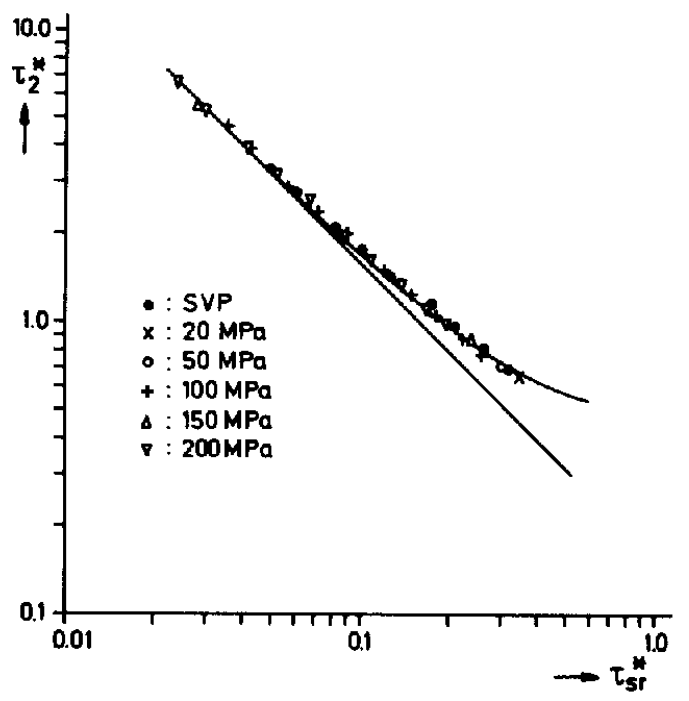

Fig. 48. Reduced rotational correlation times $\tau_{2}^{*}$ versus reduced spin-rotation correlation times $\tau_{\mathrm{SR}}^{*}$ at different constant pressures in fluid $\mathrm{CF}_{2} \mathrm{HCl}$. The straight line represents the Hubbard relation, eqn (71). ${ }^{(229)}$

the isotropic part of the spin-rotation tensor $C_{0}$, provided by molecular beam experiments, a reliable determination of $\Delta C$, the anisotropic part of the tensor was possible. ${ }^{(296,339)}$ Campbell et al. ${ }^{(340)}$ extended their NMR studies to higher densities $\left(\rho / \rho_{\mathrm{STR}}=120\right)$ and covered a large temperature range $(210 \mathrm{~K}-310 \mathrm{~K})$. At each density the temperature dependence of the ${ }^{19} \mathrm{~F}$ spin-lattice relaxation rates could be represented by an apparent activation energy $E_{p}^{+}=3.56 \pm 0.21 \mathrm{~kJ} / \mathrm{mol}$. In addition, Raman band shapes of the doubly degenerate $v_{2}(e)$ vibration-rotation band $\left(\lambda^{-1}=435 \mathrm{~cm}^{-1}\right)$ have been studied in the same $p, T$-range.

The main purpose of this study was the determination of the relationship between the angular momentum correlation times $\tau_{\mathrm{J}}$ deduced from ${ }^{19} \mathrm{~F}-T_{1}$ data and the orientational correlation times $\tau_{2}$ obtained from Raman bandshapes at intermediate gas densities. The results have been compared to predictions from the extended $J$-diffusion (ED) model ${ }^{(343)}$ and the perturbed free rotor (PFR) model ${ }^{(289)}$ of rotational dynamics. The molecular orientational correlation function $G(t)$ (Fig. 49a) deduced from Raman band shapes demonstrated the density independence of $G(t)$ at very short times $(t<0.5 \mathrm{ps})$ where orientational correlations are purely kinetic, and the increasing loss of correlations with increasing density at long times. A comparison of the corresponding orientational correlation times $\tau_{\mathrm{L}=2}$ with the angular momentum correlation times $\tau_{J}$ from the ${ }^{19} \mathrm{~F}-T_{1}$ data is also shown in Fig. 49b, together with predictions from ED and PFR models. Note that in the dilute gas limit EDJ and EDM models approach a limiting relation: ${ }^{(318)}$

$$
\tau_{2}=\tau_{\mathbf{J}} / 4
$$

which differs from the corresponding free rotor limit

$$
\tau_{2}=\tau_{\mathrm{J}} / 5
$$

due to the neglect of all the collisions except the first in the PFR models. The experimental data corroborate this linear relationship as given by the extended diffusion model.

In a related study, Finney et al ${ }^{(341)}$ and Wolfe et al ${ }^{(\mathbf{3 4 2 )}}$ have investigated the angular momentum relaxation in compressed $\mathrm{CF}_{4}$ and $\mathrm{CF}_{4} / \mathrm{Ar}$, or $\mathrm{CF}_{4} / \mathrm{Ne}$ mixtures. For this purpose ${ }^{19} \mathrm{~F}$ spin-lattice relaxation times were measured as a function of pressure and temperature over a wide density $\left(0.28 \leqslant \rho / \rho_{\mathrm{c}} \leqslant 2.48\right)$ and temperature $\left(1.2 \leqslant T / T_{\mathrm{c}} \leqslant 1.64\right)$ range. The main goal of this study was to provide a test of the rough hard sphere (RHS) model ${ }^{(139,140)}$ for angular momentum relaxation in 

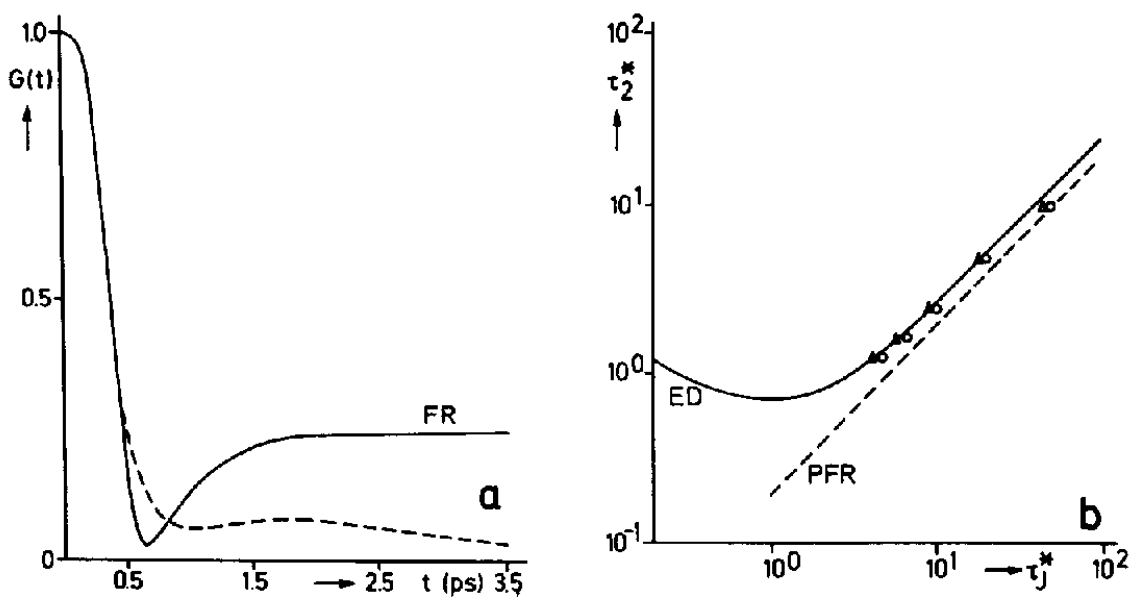

FIG.49. (a) Orientational correlation function $G(t)$ of a free rotor molecule (FR) and of carbon tetrafluoride $\left(\mathrm{CF}_{4},--\right)$ at $296 \mathrm{~K}$ and a reduced density $\rho / \rho_{\text {STP }}=120$. (b) Comparison of reduced orientational $\left(\tau_{2}^{*}\right)$ and angular momentum $\left(\tau_{f}^{*}\right)$ correlation times in carbon tetrafluoride. Predictions from extended diffusion (- EDJ) and perturbed free rotor (-- PFR) models are also shown.

dense fluids $\left(\rho / \rho_{\mathrm{c}}>2\right)$ (see eqn (72)). The emphasis focused on whether the RHS model can be extended to lower densities $\left(\rho / \rho_{\mathrm{c}}<2\right)$ and the determination of the roughness parameter $a(T)=(K+1)^{-1}$ (eqn (74)) over a large $p, T$-range. The fluorine relaxation rates have been shown to be dominated totally by the spin-rotation mechanism even at the highest densities studied. The low density measurements exhibited a temperature dependence $T_{1} / n \sim T^{-3 / 2}$ as observed for a number of molecules with a dominant spin-rotation relaxation. The isothermal pressure dependence of ${ }^{19} \mathrm{~F}-T_{1}$ is shown in Fig. 50.

Application of the RHS model affords an estimation of the effective hard sphere diameter $\sigma(T)$. It has been obtained by an iterative procedure fitting the experimental data to the expression:

$$
\frac{1}{\tau_{\mathrm{J}}}=(a(T)+b \rho) \frac{1}{\tau_{\mathrm{E}}}(\sigma),
$$

and choosing a new $\sigma$ repeatedly until $b \simeq 0$. This procedure provides the parameters $\sigma(T)$ and $a(T)$.

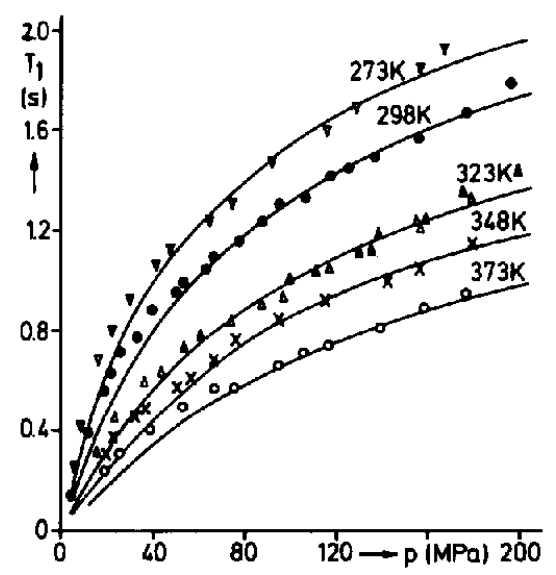

Fig. 50. Isothermal pressure dependence of the ${ }^{19} \mathrm{~F}-T_{1}$ in carbon tetrafiuoride $\left(\mathrm{CF}_{4}\right)^{(341)}$ 
The hard sphere diameter shows an essentially linear dependence on temperature and agrees well with estimates from liquid phase data. ${ }^{(302)}$ Self-diffusion data in the gas phase $\left(\rho \leqslant 2 \rho_{\mathrm{c}}\right)$, however, lead to slightly larger $(\sim 6 \%)$ diameters. ${ }^{(344)} \mathrm{A}$ decreasing hard sphere diameter with increasing temperature results from real molecules approaching each other more closely at greater kinetic energy. These temperature dependent diameters were used to estimate the roughness-parameter $a(T)$ over the whole density range. In the RHS model $a(T)$ should be rigorously independent of temperature: this model breaks down at low densities as is clearly visible in Fig. 51 . This has been attributed to the increasing importance of intermolecular attractive forces at intermediate densities which cause an increase of $\boldsymbol{g}(\boldsymbol{\sigma})$ in the Enskog collision time $\tau_{\mathrm{E}}$ over that of a hard sphere fluid.

Except for the fluoromethanes, only a few other halomethanes have been investigated by HP-NMR. From a combination of Raman and deuterium NMR experiments as a function of pressure $(p \leqslant 250 \mathrm{MPa})$ and temperature $\left(273 \mathrm{~K} \leqslant T \leqslant 363 \mathrm{~K}\right.$ ) Campbell et al ${ }^{(\mathbf{3 4 5})}$ characterized thoroughly the molecular rotational motions in liquid methyl iodide $\left(\mathrm{CH}_{3} \mathrm{I}\right)$. The density and viscosity were also determined under the same experimental conditions. Raman bandshape analysis of the $v_{3}\left(a_{1}\right)$ band of $\mathrm{CH}_{3} \mathrm{I}$ provided orientational correlation functions $G(t)$ characterizing the tumbling motion of the symmetry axis of the symmetric top molecule. Integration of the correlation function then yielded the correlation time $\tau_{2 \perp}$, hence the perpendicular component of the rotational diffusion tensor $D_{\perp}=1 / 6$ $\tau_{2 \perp}$, as the tumbling motion of liquid $\mathrm{CH}_{3} \mathrm{I}$ is certainly in the diffusion limit. The correlation functions $G(t)$ show the characteristic change-over from the free rotor behaviour at very short times $(t<0.5 \mathrm{ps})$ to the essentially exponential decay at longer times ( $t>1 \mathrm{ps}$ ). This decay decreased with decreasing temperature and/or increasing pressure. The effect of temperature at constant density on the reorientational dynamics is much smaller than combined density-temperature effects at constant pressure. The perpendicular component of the rotational diffusion tensor $D_{\perp}$ deduced from $G(t)$ is shown in Fig. 52 as a function of pressure and temperature. Apparent activation energies at constant pressure $E_{\mathrm{p}}^{*}=9.6 \mathrm{~kJ} / \mathrm{mol}(p=50 \mathrm{MPa})$, and constant density $E_{v}^{*}=5.65 \mathrm{~kJ} / \mathrm{mol}\left(\rho=2420 \mathrm{~kg} / \mathrm{m}^{3}\right)$ have been deduced. These results corroborate the general trend that the energy of activation at constant pressure and variable temperature is approximately twice as large as that observed at constant density. Clearly the low value of $E_{v}^{\#}$ at constant density raises severe doubts as to the significance of the activated process description of molecular motions in these non-associated liquids. The volume of activation $\Delta V^{*}=9 \pm 1\left(\mathrm{~cm}^{3} / \mathrm{mol}\right)$ for the tumbling motion was also derived from the various isotherms, and compares favourably with the corresponding pressure coefficient of the shear viscosity. Hence the more restricted tumbling motion of the symmetry axis is closely coupled to the shear viscosity, as expected from hydrodynamics.

Information about the fast spinning motion around the $C_{3}$-axis was deduced from the deuterium spin-lattice relaxation rate. Assuming an axially symmetric electric field gradient with the largest

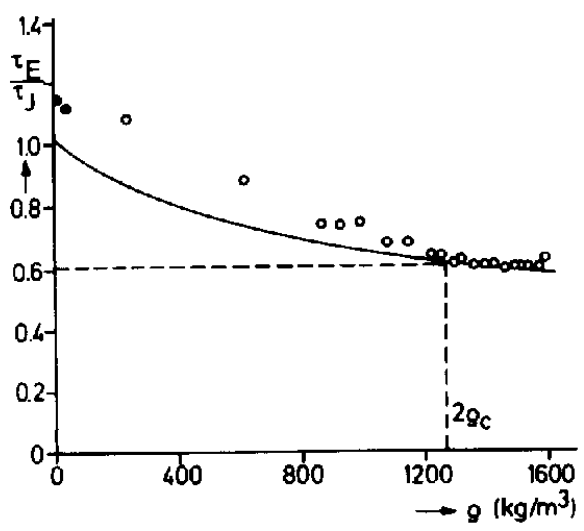

Fig. 51. Comparison of angular momentum $\left(\tau_{J}\right)$ and corresponding Enskog $\left(\tau_{\mathbb{E}}\right)$ correlation times versus density in $\mathrm{CF}_{4}{ }^{(341)}$ 


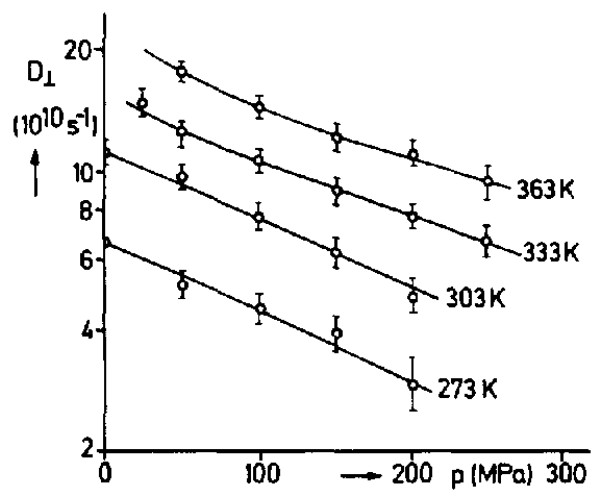

FIG. 52. Perpendicular component of the rotational diffusion tensor $\mathrm{D}_{\perp}$ of methyl iodide $\left(\mathrm{CH}_{3} \mathrm{I}\right)$ as a function of pressure and temperature. ${ }^{(345)}$

component $q_{z z}$ along the $C-D$ bond axis, an effective integral reorientational correlation time $\tau_{\text {eff }}$ was obtained (eqn 39). Within a rotational diffusion model the parallel component of the diffusion tensor $D_{\|}$was determined, though a $\chi$-test as proposed by Huntress ${ }^{(346)}$ indicated the importance of inertial effects throughout the pressure range investigated. Further, in cases where $\tau_{2 \perp} / \tau_{\text {eff }} \gg 1$ the calculated $D_{\|}$depend strongly on the precise value of the angle $\theta$ in eqn (104). The rather unphysical increase of $D_{\|}$with pressure led Campbell et al. ${ }^{(345)}$ to suggest that the $z$-axis of the principal frame of the electric field gradient-tensor deviates slightly $\left(<4^{\circ}\right)$ from $C-D$ bond axis in accord with similar findings reported in the literature ${ }^{(345)}$ As the use of a diffusion model to characterize the fast spinning motion is at best only qualitative, the authors also investigated the rotational Langevin model ${ }^{(329-331,348)}$ in the high and low friction limit. The temperature dependence of the methyl group rotation follows an approximate $T^{1 / 2}$ relationship, indicative of an inertial reorientation process though with significant frictional torques hindering the free rotation of the $\mathrm{CH}_{3}$-group. Increasing pressure, however, does not affect the spinning motion of the methyl group, implying little change of the torques with density under the conditions of the experiment $\left(2200 \mathrm{~kg} / \mathrm{m}^{3} \leqslant \rho \leqslant 2500 \mathrm{~kg} / \mathrm{m}^{3}\right)$.

Van der Hart ${ }^{(349)}$ reported a very careful HP-NMR investigation of the molecular reorientation in liquid deutero-chloroform $\left(\mathrm{CDCl}_{3}\right)$. He measured the deuterium NMR spin-lattice relaxation of neat liquid $\mathrm{CDCl}_{3}$ over the range $300 \mathrm{~K}<T<440 \mathrm{~K}$ and at pressures up to $500 \mathrm{MPa}$. Further HP-NMR of this substance are reported by Nolle and $\mathrm{Mahendroo}{ }^{(350)}$ on $\mathrm{CHCl}_{3}$, and Campbell and Jonas ${ }^{(351)}$ who compared their deuterium $T_{1}$ measurements with related Raman bandshape studies. These investigations have been performed at room temperature only.

Since the deuterium nucleus is on the symmetry axis $\left(C_{3}\right)$, the deuterium relaxation monitors the tumbling motion of this axis only. With the quadrupolar coupling constant (QCC) determined for solid $\mathrm{CDCl}_{3}{ }^{\left({ }^{(352)}\right.}$ integral correlation times $\tau_{2 \perp}$ were obtained directly from the experimental $T_{1}$ and compared to various models of reorientational motions. Figure 53 compares the isochoric temperature dependence and the isothermal volume dependence of the relaxation times $T_{1}$.

Within the realm of activation models the correlation times $\tau_{2 \perp}$ are connected to the activation energy $\Delta E^{*}$ and activation volume $\Delta V^{*}$ by:

$$
\tau_{2 \perp}=A(T) \exp \left[\left(\Delta E^{\#}+p \Delta V^{\#}\right) / R T\right],
$$

with the activation parameters $\Delta E^{\#}, \Delta V^{\#}$ and $\Delta H^{\#}=\Delta E^{\#}+p \Delta V^{*}$ being independent of $p, V$ and $T$. Transition state theory ${ }^{(353)}$ predicts a linear temperature dependence of the pre-exponential factor $A(T)$, which is, however, almost always neglected. Whereas $\Delta H^{*}$ and $\Delta V^{*}$ may be obtained from the slopes of $\ln T_{1}$ versus $1 / T$ or $p$ respectively, the equation of state has to be known to deduce $\Delta E^{*}$ from the isochoric $T$-dependence of $T_{1}$ according to eqn (16). Considering Fig. 53, it is clear that both $\Delta V$ * and $\Delta E^{*}$ are functions of pressure and temperature. As found for other liquids, ${ }^{(354,355)}$ the slope of 

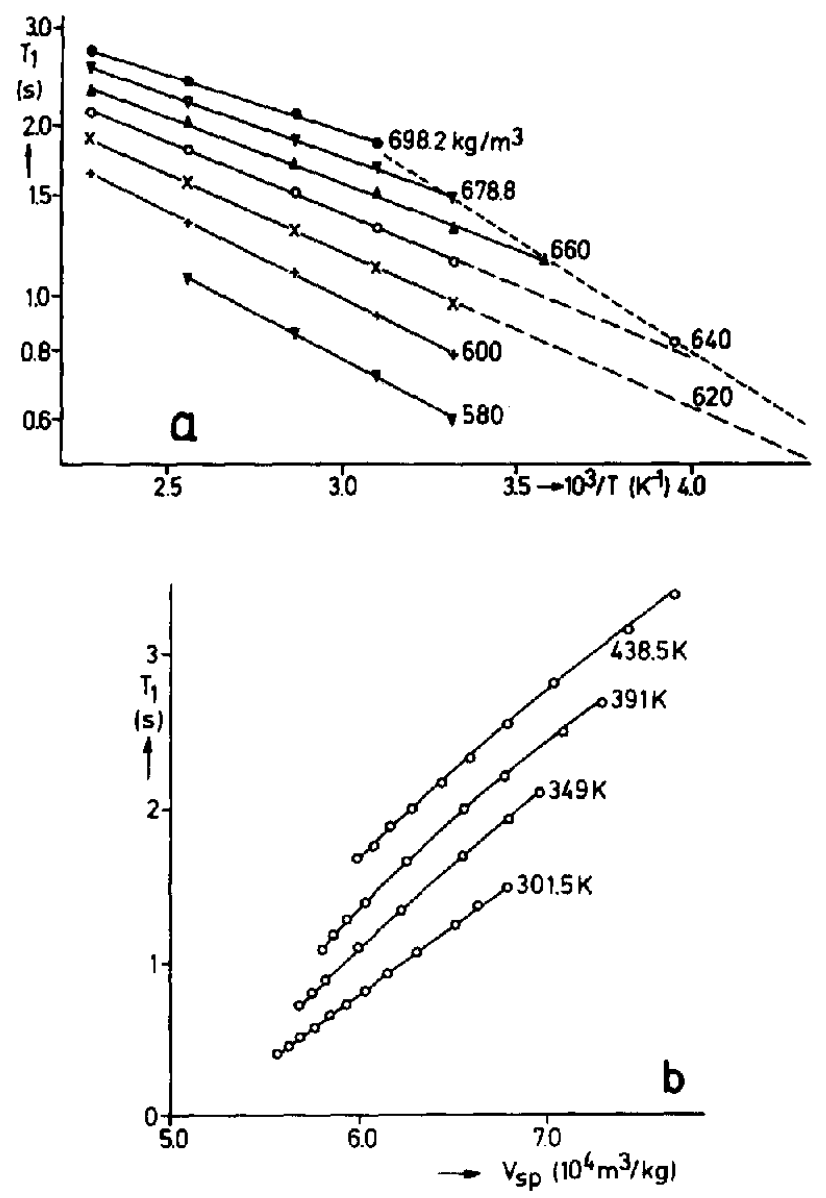

FiG. 53. Comparison of (a) the isochoric temperature and (b) the isothermal volume dependence of the deuterium $\left({ }^{2} \mathrm{H}\right)$ spin-lattice relaxation time in deutero-chloroform. ${ }^{(349)}$

$\ln T_{1}$ versus $p$ at constant temperature decreases with increasing pressure and becomes a constant $\left(\Delta V^{\#}=5.8 \pm 0.2 \mathrm{~cm}^{3} / \mathrm{mol}\right)$ at high pressures $p>200 \mathrm{MPa}$. Also $\Delta V^{\#}$ decreases slightly at high temperatures $\left(\Delta V^{\#}=4.8 \pm 0.3 \mathrm{~cm}^{3} / \mathrm{mol}\right)$. Hence, at high pressures the activated jump picture of reorientational motions apparently is better for describing the $T_{1}$ isotherms. This might be construed as evidence that a high density liquid achieves a greater amount of angular and positional correlation. As the activation enthalpy $\Delta H^{\#}$ is found to be a linear function of pressure, the activation volume has to be $\Delta V^{\#}=8.0 \pm 0.2 \mathrm{~cm}^{3} / \mathrm{mol}$ for the activation energy $\Delta E^{\#}$ to be constant. As the actual $\Delta V^{\#}$ is considerably smaller the activation energy also exhibits a considerable pressure dependence. Figure 53 demonstrates that $\Delta E^{\#}$ is also a function of volume. Hence the variation of both $\Delta V^{\#}$ and $\Delta E^{\#}$ as a function of the thermodynamic state variables points to the inadequacy of the activated state picture of reorientational motions in simple dense liquids.

Free volume theories when applied to the HP-NMR data of chloroform also show definite shortcomings. According to the suggestion of Batchinski ${ }^{(356)}$ the relation $\eta \sim 1 / V_{f}$ should hold with $V_{\mathrm{f}}=V-V_{\mathrm{o}}$ a measure of free volume, and with the Debye-Stokes-Einstein formula as modified by McClung and Kivelson, ${ }^{(357)} \tau_{2} \sim \kappa \eta / T$, so that the experimental relaxation time $T_{1}$ should then be a linear function of volume $V$ at constant temperature

$$
T_{1} \sim \tau_{2}^{-1} \sim(\kappa \eta / T)^{-1} \sim V-V_{0} .
$$


This, however, is not observed (see Fig. 53). The rotation-translation coupling parameter $\kappa$ is often assumed to be constant, but is found to decrease with increasing temperature at constant density and to decrease with increasing density at constant temperature. Theoretically $k$ is related to anisotropic intermolecular potentials, and hence depends strongly on non-spherical molecular shapes, as has been demonstrated conclusively by Fury and Jonas. ${ }^{(358)}$ Also, the more sophisticated expression derived by Cohen and Turnbull(359) does not fit the $T_{1}$ data, since $T_{1}$ is not constant at constant volume. Instead van der $\mathrm{Hart}^{\left({ }^{(349)}\right.}$ proposed a relation

$$
T_{1}=C(T)\left[\rho-\rho_{\mathrm{o}}(T)\right]
$$

as the experimental $T_{1}$ data show a linear density dependence for all temperatures. In summary, free volume models are also not able to describe the reorientational dynamics of simple liquids when tested over wide ranges of temperature and density.

Simple cell models assume the molecules are trapped in a cage with moveable walls of infinite potential $^{(360)}$ and give the mean time between collisions as:

$$
\tau_{\text {coll }}=\frac{\langle l\rangle}{\langle v\rangle}=\left(A / T^{1 / 2}\right)\left(V_{\mathrm{m}}^{1 / 3}-\sigma\right),
$$

with $V_{\mathrm{m}}$ the average molecular volume and $\sigma$ an effective hard sphere diameter. Assuming that the angular momentum correlation time $\tau_{\mathrm{J}}$ is proportional to the average time between collisions, then:

$$
\tau_{\mathrm{J}}=Z \tau_{\mathrm{col1}}
$$

$\tau_{\mathrm{J}}$ has been calculated from $\tau_{2 \perp}$ with the $J$-diffusion model ${ }^{(132)}$. According to eqn (113) and eqn (112) the reduced angular momentum correlation times $\tau_{\mathrm{J}}^{*}$ should be linear in $V^{1 / 3}=\langle R\rangle$, and independent of temperature. Figure 54 demonstrates the failure of the simple cell model to account for the observed behaviour of the $\tau_{j}^{*}$ data. The constant temperature data are neither linear in $V^{1 / 3}$ nor independent of temperature. In addition, the slope of the $\tau_{j}^{*}$ versus $V^{1 / 3}$ plot is considerably smaller than predicted by the cell model in the constant temperature plots, but much closer to the theoretical value in the constant pressure diagram. Figures $54 \mathrm{a}$ and $\mathrm{b}$ clearly demonstrate that the effective hard sphere diameter is a function of temperature and pressure respectively. Obviously the molecules are not infinitely hard, and as a result, the activated jump model, the free volume theories, or the cell model of a liquid do not fit the whole range of experimental HP-NMR data in liquid chloroform satisfactorily as has been demonstrated convincingly by van der Hart. ${ }^{(349)}$

A similar molecular system, liquid $\mathrm{CFCl}_{3}$, has been investigated by De Zwaan and Jonas ${ }^{(361)}$ to provide an experimental test of the rough hard sphere (RHS) model of liquids by HP-NMR. ${ }^{19} \mathrm{~F}$ spin-lattice relaxation times, self-diffusion coefficients and densities have been measured for liquid $\mathrm{CFCl}_{3}$ at temperatures of $T=341 \mathrm{~K}, 379 \mathrm{~K}$ and $460 \mathrm{~K}$, and at pressures up to $p \leqslant 200 \mathrm{MPa}$. The purpose of their study was twofold. Firstly to determine the best effective hard sphere diameter for $\mathrm{CFCl}_{3}$ by using the density dependence of the spin-rotation relaxation rate $R_{1}$, sR $\left({ }^{19} \mathrm{~F}\right)$. Secondly to compare the diffusion coefficient of $\mathrm{CFCl}_{3}$ to those predicted from the RHS model using the hard sphere diameter deduced from relaxation data. The experimental relaxation times $T_{1}\left({ }^{19} \mathrm{~F}\right)$ are shown in Fig. 55a. As the spin-lattice relaxation of the ${ }^{19} \mathrm{~F}$ nucleus is dominated completely by the spin-rotation interaction, angular momentum correlation times $\tau$, were obtained directly from the experimental $T_{1}$ with eqn (68), and the spin-rotation coupling $C_{\text {eff }}^{2}$ as given by Gillen et al., ${ }^{(362)}$ and McClung. ${ }^{(363)}$ Equations (72) and (73) then provide the necessary connection with the RHS model. The hard sphere diameter $\sigma$ and the roughness parameter $a(T)$ were determined by plotting $\ln T_{1, \mathrm{sR}}$ against $\ln \rho$ and matching the curvature to a plot of $\ln (Z-1)$ against $\ln \eta$, with $Z=1+4 \eta g(\sigma)$ the compressibility factor of the hard sphere system, and $\eta=\pi / 6 n \sigma^{3}$ the packing fraction. The reduced angular momentum correlation time $\tau_{\mathrm{j}}^{*}$ should be independent of temperature at constant number density $n$. The experiments, however, showed an increase of $\tau_{j}^{*}$ with temperature leading to an effective hard sphere diameter $\sigma(T)$ which decreases with increasing thermal energy, as would be expected for more realistic intermolecular potentials. An equivalent result was obtained by fitting the isothermal density dependence of $T_{1}$, sR, which also required $\sigma(T)$ to decrease slightly with increasing temper- 

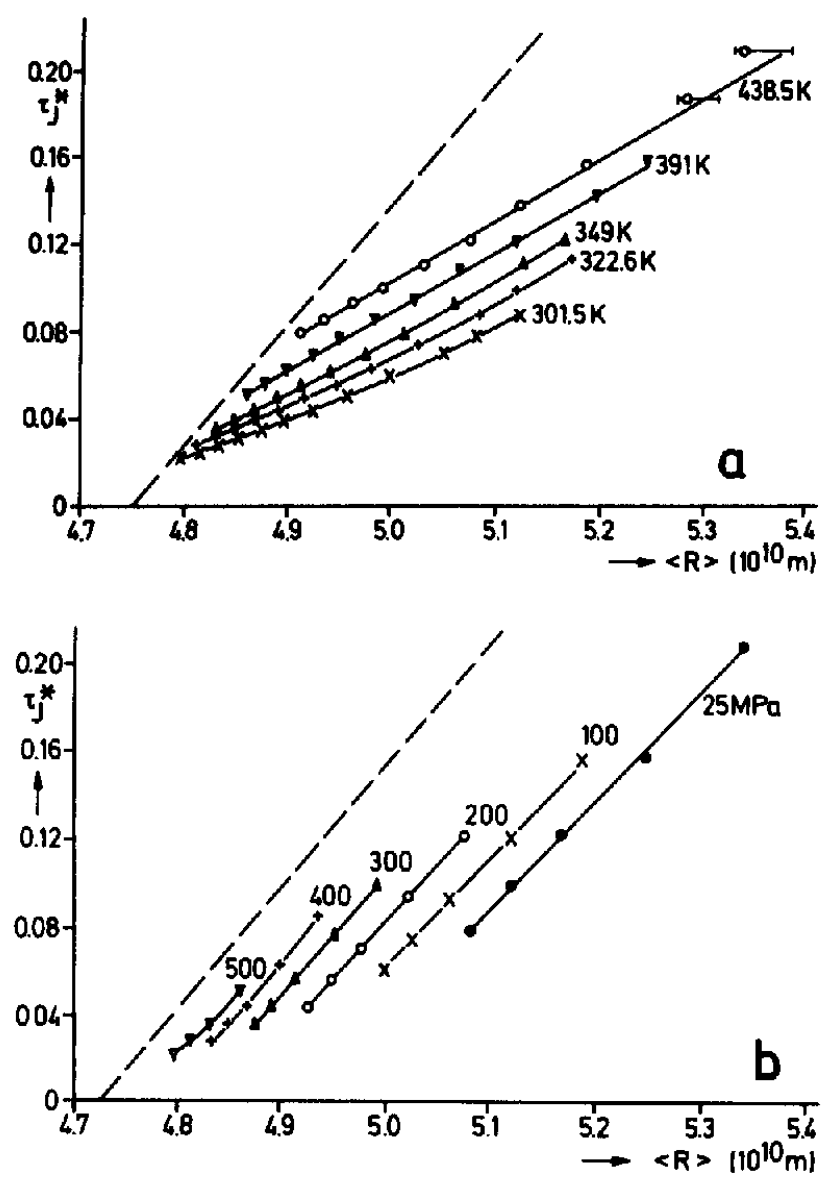

Fig. 54. (a) Reduced angular momentum correlation times $\tau_{j}^{*}$ versus average distance $\langle R\rangle$ of the $\mathrm{CDCl}_{3}$ molecules at constant temperature. The prediction of the cell model is indicated by the dashed line. (b) Corresponding isobaric dependence of $\tau^{*}$ on $\langle R\rangle^{(349)}$

ature. From these findings De Zwaan and Jonas ${ }^{(361)}$ came to the conclusion that the roughness parameter $a(T)$ is not strongly temperature dependent and that its value is close to the perfectly rough hard sphere limit (see eqn (74)). The self-diffusion coefficient $D$ is related to the linear momentum correlation time $\tau_{\mathrm{P}}$ which, in turn, is assumed to be proportional to the Enskog relaxation time $\tau_{\mathrm{E}}$ giving:

$$
D=\frac{k T}{m} \tau_{\mathrm{P}}=a(T) \frac{k T}{m} \tau_{\mathrm{E}}=a(T) D_{\mathrm{E}} .
$$

Hence angular momentum $\left(\tau_{\mathrm{j}}\right)$, and linear momentum $\left(\tau_{\mathrm{p}}\right)$ correlation times may be related to the same dynamic quantity, the Enskog collision time $\tau_{\mathrm{E}}$, grasping the essence of the binary collision approximation in a hard spheres liquid. If the latter holds in a real fluid the ratio $D / D_{E}$ must be independent of density or packing fraction $\eta$ at constant temperature. Experimentally, a decrease of $D / D_{\mathrm{E}}$ with increasing density was observed (Fig. 55b) and was found to be in good accord with molecular dynamics (MD) simulations of the hard spheres fluid. ${ }^{(49,50)}$ This issue has been discussed already at length in connection with self-diffusion measurements by HP-NMR techniques. It illustrates again the importance of density effects on transport properties in liquids. 

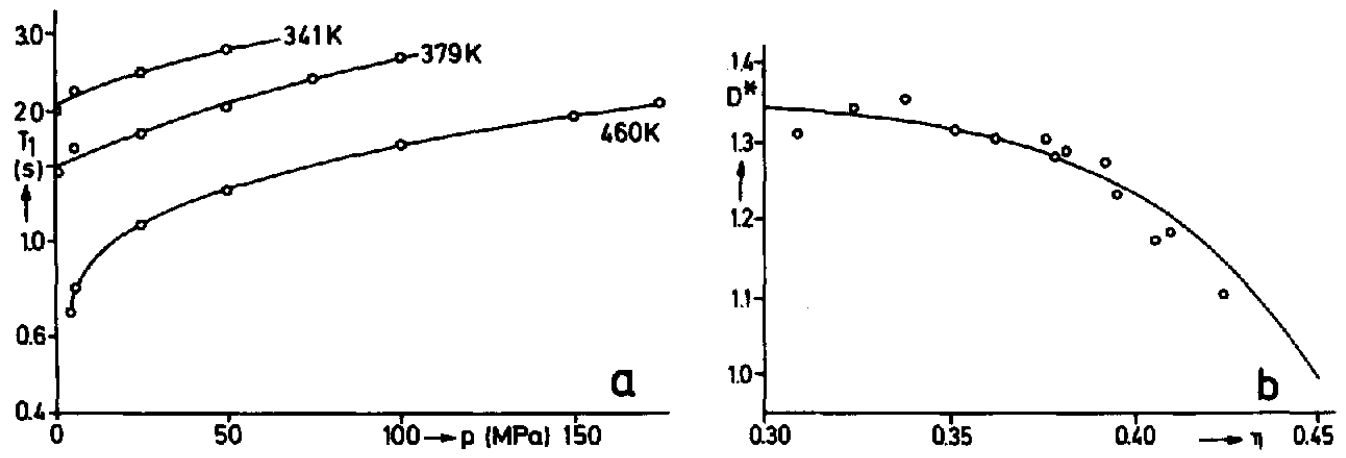

FiG. 55. (a) Isothermal pressure dependence of ${ }^{19} \mathrm{~F}-T_{1}$ in $\mathrm{CFCl}_{3}$. (b) Reduced self-diffusion coefficient $D^{*}=D / D_{\mathrm{E}}$ versus packing fraction $\eta$ in $\mathrm{CFCl}_{3} . D_{\mathrm{E}}$ is the Enskog diffusion coefficient. ${ }^{(361)}$

A molecular system closely related to the halomethanes is liquid sulphur hexafluoride $\mathrm{SF}_{6}$ which has been investigated by HP-NMR by De Zwaan and Jonas. ${ }^{(364)}$ Again ${ }^{19} \mathrm{~F}$ spin-lattice relaxation times, self-diffusion coefficients and densities have been determined as a function of temperature $\left(0.8 \leqslant T / T_{\mathrm{c}} \leqslant 1.5\right)$ and density $\left(0.4 \leqslant \rho / \rho_{\mathrm{c}} \leqslant 2.6\right)$. Since $\mathrm{SF}_{6}$ is a spherically symmetric molecule and its spin-lattice relaxation is dominated by the spin-rotation interaction, angular momentum correlation times $\tau_{\mathrm{J}}$ could again be obtained over a wide density and temperature range using the EDJ model ${ }^{(132)}$ and the isotropic and anisotropic components of the spin-rotation tensor as reported by Sunder and McClung $^{(365)}$ and Courtney and Armstrong. ${ }^{(339)}$ The latter authors reported ${ }^{19} \mathrm{~F}$ spin-relaxation measurements of $\mathrm{SF}_{6}$ in the dilute gas phase at densities $0.015 \leqslant \rho / \rho_{\mathrm{SPT}} \leqslant 20$ and at temperatures $240 \mathrm{~K} \leqslant T \leqslant 350 \mathrm{~K}$. At room temperature a $T_{1}$ minimum was observed at low densities, which allowed the determination of the spin-rotation tensor components. The $T_{1}$ data have been analyzed in much the same way as has been discussed in case of $\mathrm{CF}_{4}$, which has also been studied by these authors and hence the discussion will not be repeated here.

De Zwaan and Jonas ${ }^{(364)}$ analyzed their $\tau_{\mathrm{J}}$-data within the RHS model and obtained effective hard sphere diameters $\sigma(T)$ as discussed above for the case of liquid $\mathrm{CFCl}_{3}$. The value of $\sigma(T)$ decreases slightly with increasing temperature as is generally found in real liquids because the slope of the repulsive potential is large but not infinite. These values have been used to calculate spin-lattice relaxation rates via eqn (72). The latter have been found to reproduce the experimental data very well (Fig. 56) at densities $\rho>2 \rho_{\mathrm{c}}$ where the RHS model is expected to be a valid approximation of a real

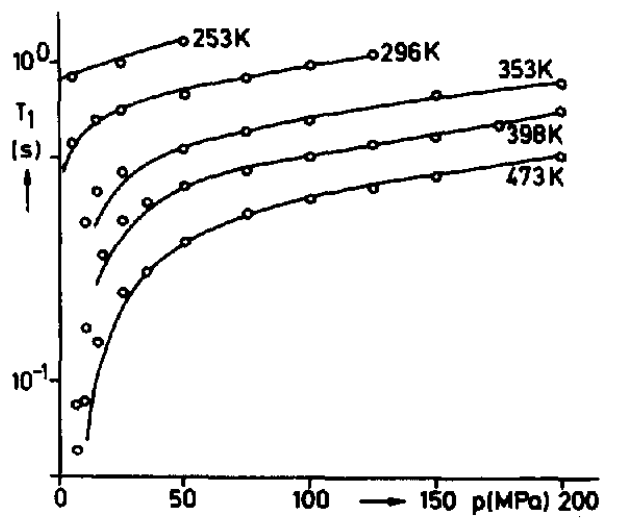

FIG. 56. Isothermal pressure dependence of the ${ }^{19} \mathrm{~F}-T_{1}$ in sulphur hexafluoride $\mathrm{SF}_{6}{ }^{(364)}$ 
liquid. At lower densities the experimental relaxation rates are smaller than predicted by the RHS model indicating that the time between collisions becomes smaller due to the increasing importance of attractive interactions at intermediate densities. The density dependence of the self-diffusion coefficient is also well reproduced with the RHS model with $D_{\text {SHS }}$ as given by Dymond. ${ }^{(366)}$ A rotation-translation coupling parameter $A \simeq 0.97$ was found, indicating that $\mathrm{SF}_{6}$ corresponds very closely to a smooth hard spheres fluid in the density and temperature range investigated.

Hauer et al. ${ }^{134}$ investigated another sulphur containing compound, hydrogen sulphide $\mathrm{H}_{2} \mathrm{~S}$ and its deuterated analogue by HP-NMR. They measured the pressure and temperature dependence of the spin-lattice relaxation time $T_{1}$ of the protons $\mathrm{H}_{2} \mathrm{~S}$ and the deuterons in $\mathrm{D}_{2} \mathrm{~S}$ between the melting pressure curve and $395 \mathrm{~K}$ and at pressures of $p \leqslant 200 \mathrm{MPa}$. Other NMR investigations, though under ambient pressure only, have been reported in the solid phases ${ }^{(367-369)}$ and in the liquid. ${ }^{(370-373)}$

Liquid hydrogen sulphide, the higher homologue of water, does not reveal any hydrogen bonding ability. The molecular interactions are dominated by dipole and dispersion forces. ${ }^{(374,375)}$ Its molecular geometry and dipole moment is however similar to water. ${ }^{(376,377)}$

The spin-lattice relaxation of the protons in hydrogen sulphide has contributions from dipolar interactions at low temperatures and spin-rotation interactions at high temperatures. However, none of these really dominate in the $p, T$-range investigated. In contrast, for deuterated hydrogen sulphide, electric quadrupole interactions dominate the relaxation of the ${ }^{2} \mathrm{H}$ nuclei at low temperatures but contributions from spin-rotation interactions cannot be excluded a priori, though the relaxation time $T_{1}\left({ }^{2} \mathrm{H}\right)$ increases with temperature over most of the $p, T$-range covered by the experiments (Fig. 57). With eqn (55) and eqn (68) the total relaxation rate $R_{1}\left({ }^{2} \mathrm{H}\right)$ in $\mathrm{D}_{2} \mathrm{~S}$ is seen to be proportional to an integral orientational correlation time $\tau_{2}$ and an angular momentum correlation time $\tau_{\text {, }}$ if hydrogen sulphide is treated as a spherical top and if anisotropic components of the spin-rotation tensor $C$ may be neglected. As $\tau_{2}$ and $\tau_{j}$ are interrelated, according to various models of rotational motions in liquids and gases, ${ }^{(86,133,137,139,318,378)} \tau$, is left as the only adjustable parameter which can be obtained from the experimental rates $R_{1}\left({ }^{2} \mathrm{H}\right)$ directly. To treat both relaxation rates selfconsistently, spin-rotation $\left(R_{1}, \mathrm{sR}\right)$ and intramolecular dipolar $\left(R_{1, \text { intra }}\right)$ relaxation contributions to the proton relaxation rates have been calculated from the corresponding rates $R_{1}$. SR $\left({ }^{2} \mathrm{H}\right)$ and $R_{1 \mathrm{Q}}\left({ }^{2} \mathrm{H}\right)$ respectively, assuming that the reduced correlation times $\tau_{2}^{*}$ and $\tau_{\mathrm{j}}^{*}$ are identical for both molecules, $\mathrm{H}_{2} \mathrm{~S}$ and $\mathrm{D}_{2} \mathrm{~S}$ respectively, at constant pressure and temperature. This assumption posed severe constraints upon the different motional models tested and only the extended $J$-diffusion model has been found to provide a consistent representation of both relaxation rate curves $R_{1}\left({ }^{1} \mathrm{H}\right)$ and $R_{1}\left({ }^{2} \mathrm{H}\right)$ over the whole $p, T$-range investigated. The separation of the various relaxation contributions is shown in Fig. 58 . It is seen that intermolecular dipolar interactions modulated by positional fluctuations make the largest contribution to the proton relaxation close to the melting temperatures. This is a consequence of the fast rotational motions of the molecules in liquid hydrogen sulphide even close to $T_{\mathrm{m}}(p)$ resulting in

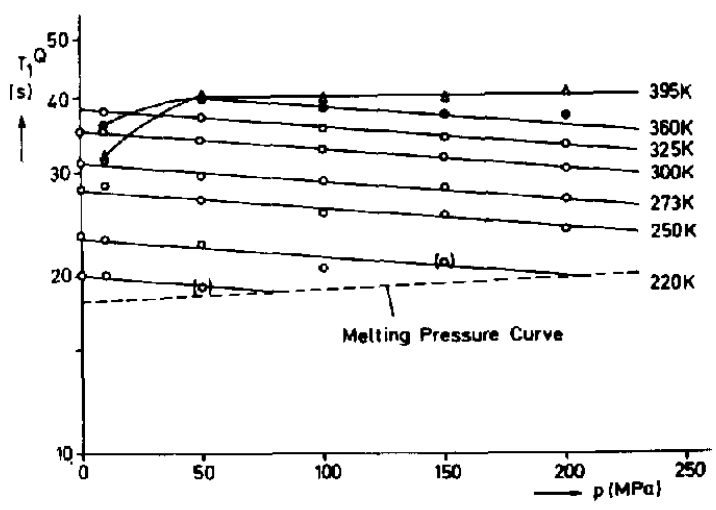

FIG. 57. Isothermal pressure dependence of the ${ }^{2} \mathrm{H}-T_{1}$ in deuterated hydrogen sulphide $D_{2} S^{(134)}$ 

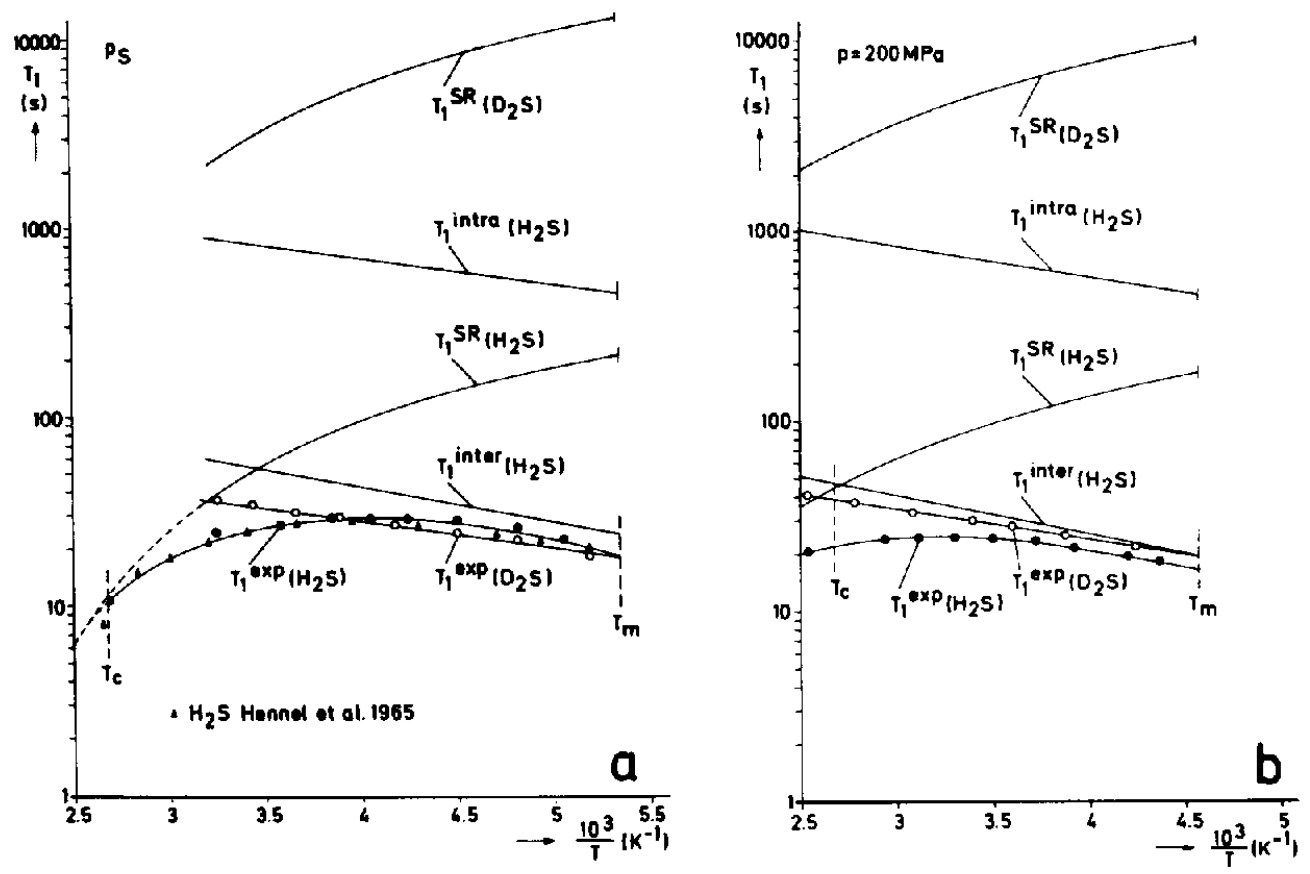

FIG. 58. Temperature dependence of the relaxation times $T_{1}$ in (a) $\mathrm{H}_{2} \mathrm{~S}(\bullet)$ and (b) $\mathrm{D}_{2} \mathrm{~S}(\mathrm{O})$ at saturation pressure $p_{s}$ and $200 \mathrm{MPa}$. Estimated contributions from different relaxation mechanisms are also shown. ${ }^{(134)}$

very short orientational correlation times $\tau_{2}^{*}$ hence small intramolecular dipolar relaxation rates. It is unfortunate that no self-diffusion coefficient data yet exist for liquid hydrogen sulphide which would provide a further stringent test of the motional models considered. The relative magnitude $Q=\left(R_{1, \text { inter }}\right) /\left(R_{1, \text { intra }}\right) \sim \tau_{\mathrm{d}} / \tau_{2}$ of inter- and intramolecular dipolar relaxation rates in hydrogen sulphide contrasts markedly with the corresponding ratio in liquid ammonia and liquid water at their respective melting points $T_{\mathrm{m}}\left(p_{\mathrm{s}}\right)$ giving:

$$
\begin{array}{rrr}
\mathrm{H}_{2} \mathrm{~S} & \mathrm{NH}_{3} & \mathrm{H}_{2} \mathrm{O} \\
Q=18 & 1.8 & 0.5
\end{array}
$$

Clearly, reorientational motions are slowed down strongly in the hydrides of nitrogen and oxygen compared to those of sulphur due to the hydrogen bonding ability of the former. This is further corroborated by a comparison of reduced reorientational correlation times $\tau_{2}^{*}$

$$
\begin{array}{ccccl} 
& \mathrm{D}_{2} \mathrm{~S} & \mathrm{ND}_{3} & \mathrm{D}_{2} \mathrm{O} & \\
\tau_{2}^{*} & 1.03 & 6.5 & 86 & T=T_{\mathrm{m}}, p_{\mathrm{s}} \\
& 0.89 & 3.8 & 12 & T=0.56 T_{\mathrm{c}}, p_{\mathrm{s}} .
\end{array}
$$

Experimental activation enthalpies $\Delta H^{*}$ (eqn (109)) deduced from the isobars $R_{1 \mathrm{Q}},\left(R_{1, \text { intra }}\right)$ range from 3-4 kJ/mol, hence are comparable to the mean thermal energy in the range of temperatures covered by the experiment indicating that the activated jump model of transport is certainly not applicable to liquid hydrogen sulphide. Rzany and Sciesinski ${ }^{(375)}$ estimated an interaction energy of $E=1.9[\mathrm{~kJ} / \mathrm{mol}]$ due to permanent dipole interactions. Thus, these interactions contribute significantly to the hindrance of rotational motions in liquid hydrogen sulphide. As the permanent dipole moments of $\mathrm{H}_{2} \mathrm{O}\left(1.8 \times 10^{-18} \mathrm{esu}\right)$ and $\mathrm{NH}_{3}\left(1.3 \times 10^{-18} \mathrm{esu}\right)$ are not significantly different from that 
of $\mathrm{H}_{2} \mathrm{~S}\left(1.1 \times 10^{-18} \mathrm{esu}\right)$ the much higher barriers to rotation in the former liquids again supports the importance of hydrogen bonding interactions upon rotational motions. Further experimental densities for hydrogen sulphide are lacking at pressures substantially higher than $p_{\mathrm{s}}$.

Figure 59 compares the density dependence of $\tau_{j}^{*}$ along the coexistence curve with predictions from the RHS model. The effective hard sphere diameter $\sigma \simeq 3.95 \AA-4.0 \AA$ corresponds closely to estimates of this quantity from other sources. ${ }^{(134)}$ Thus it seems that the rough hard sphere fluid represents a useful approximation to real liquid hydrogen sulphide. In the absence of a detailed discussion of isothermal density effects upon molecular motions, experimental activation volumes $\Delta V^{*}$, deduced from the isothermal pressure dependence of relaxation coefficients, may still provide some insight into transport processes in liquid hydrogen sulphide. In the low temperature region $(T \leqslant 330 \mathrm{~K}), \Delta V^{*}$ is independent of pressure and amounts to $\Delta V_{\mathrm{rol}}^{*}=2 \pm 0.5\left(\mathrm{~cm}^{3} / \mathrm{mol}\right)$ and $\Delta V_{\text {trans }}^{*} \pm 1\left(\mathrm{~cm}^{3} / \mathrm{mol}\right)$. As $\Delta V^{*}$ may be regarded as a qualitative measure of the space required by a molecule to reorientate or translate in the cage of its next neighbours translational motions are slowed down faster with increasing density than are rotational motions. This is generally observed in non-associated liquids.

6.2.2. Miscellaneous Non-Associating Organic Liquids. Some of the earliest HP-NMR experiments reported in the literature are the proton relaxation time studies of water, methyl iodide, ethyl iodide, $n$-pentane, $n$-hexane and toluene by Benedek and Purcell ${ }^{(379)}$ at pressures up to $1 \mathrm{GPa}$. Except for water, the samples were not degassed. Hence the corresponding ${ }^{1} \mathrm{H}-T_{1}$ data are largely unreliable in their absolute value though a correct physical explanation of their data has been given in that pressure effects the freedom to migrate much more than the freedom to rotate. During the $70 \mathrm{~s}$, several simple organic liquids, mainly aromatic molecules, were investigated by HP-NMR. Bull and Jonas ${ }^{(380)}$ reported the pressure dependence $(p \leqslant 220 \mathrm{MPa})$ at ambient temperature $(T=296 \mathrm{~K})$ of the proton spin-lattice relaxation time in neat liquid acetone and mixtures with its deuterated analogue. Their interest focused on the effect of pressure upon translational $\left(\sim R_{1}\right.$, inter $)$ and rotational $\left(\sim R_{1}\right.$, intra $)$ molecular motions.

The separation of inter- and intramolecular dipolar contributions to the total proton relaxation rate is affected by the isotopic dilution method.$^{(381)}$ The corresponding normalized relaxation times are shown in Fig. 60. A comparison with the normalized shear-viscosities demonstrates convincingly that the simple hydrodynamic approximation to the correlation times $\tau$ as proposed in the BPPtheory is only appropriate to the translational correlation time as discussed by Torrey. ${ }^{(382)}$ It was

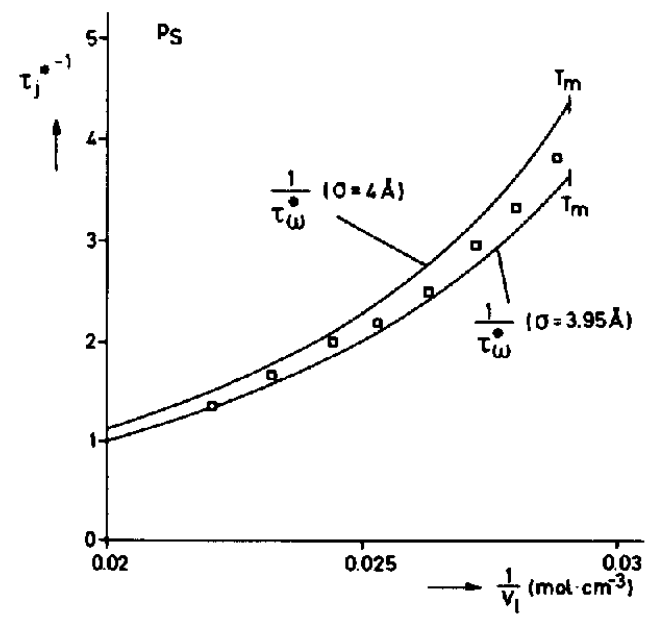

FIG. 59. Density dependence of the reduced angular momentum correlation time $\tau_{j}^{*}$ along the coexistence curve in $\mathrm{H}_{2} \mathrm{~S}$. Full lines represent predictions from the rough hard spheres model. ${ }^{(134)}$ 


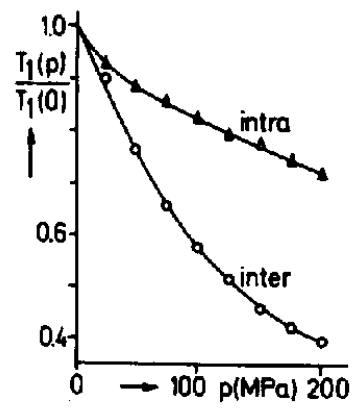

Fig. 60. Pressure dependence of intra- and intermolecular dipolar relaxation times $T_{1}$ in liquid acetone at $298 \mathrm{~K}^{(380)}$

demonstrated also that increasing density affects translational motions much stronger than reorientational motions which consist of overall tumbling motions and internal methyl group reorientations. The non-linearity of the $\ln T_{1}$ versus $p$ plots further corroborates the limited value of the concept of the activation volume in describing liquid state dynamics. ${ }^{(382,383)}$ In a subsequent investigation Bull and Jonas $^{(384)}$ reported the pressure dependence $(p \leqslant 200 \mathrm{MPa})$ at room temperature $(T=296 \mathrm{~K})$ of the deuteron and nitrogen spin-lattice relaxation times of liquid acetonitrile- $\mathrm{d}_{3}$. The relaxation of both nuclei is almost exclusively due to intramolecular quadrupole interactions and thus monitors the anisotropy of reorientational motions of the symmetric top molecule acetonitrile. The data have been interpreted in terms of the anisotropic rotational diffusion model, ${ }^{(346)}$ though inertial effects certainly play a prominent role in the case of the reorientation about the main symmetry axis $\left(C_{3}\right.$ axis). The tumbling of the symmetry axis is reflected in the ${ }^{14} \mathrm{~N}-T_{1}(p)$ yielding $D_{\perp}$ as this motion is in the diffusion limit under the conditions of the experiment. Inserting these values into eqn (104) allows the corresponding rotational diffusion coefficient $D_{\|}$to be obtained from the respective ${ }^{2} \mathrm{H}-T_{1}(p)$ data. Because of the large motional anisotropy $\left(\rho \gg 1\right.$ in eqn (104)) of acetonitrile the diffusion coefficients $D_{\|}$ could not be deduced with high precision. ${ }^{(385)}$ Furthermore, the value obtained for $D_{\|}$represents only a rough approximation to the microscopic dynamics of the fast spinning motion because of the neglect of inertial effects. The effect of pressure, shown in Fig. 61, on these motions should, however, be qualitatively correct. It was found that increasing density does not affect the fast spinning motion, indicating that reorientations around the symmetry axis do not alter the dynamic structure of liquid acetonitrile to any large extent.

Nolle and Mahendroo ${ }^{(350)}$ measured the proton $T_{1}$ in several organic liquids, including $n$-heptane, for temperatures in the range $260 \mathrm{~K}$ to $310 \mathrm{~K}$ and at pressures up to $140 \mathrm{MPa}$. Although these authors

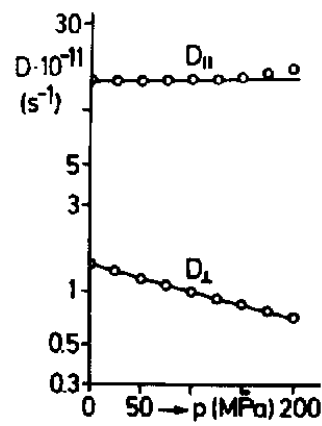

FiG. 61. Pressure dependence of the components $D_{\|}$and $D_{1}$ of the rotational diffusion tensor in liquid acetonitrile at $298 \mathrm{~K}^{(384)}$ 
noted the effect of dissolved oxygen in shortening proton relaxation times and attempted HP-NMR measurements with degassed samples, they apparently were not totally successful, as their $T_{1} \mathrm{~s}$ are considerably shorter than those reported by Powles and Gough, ${ }^{(382)}$ Lees et al. ${ }^{(386)}$ and Kashaev et al. ${ }^{(387)}$ Powles and Gough ${ }^{(382)}$ reported the pressure and temperature dependence of proton spin relaxation in liquid $n$-heptane over the range $297 \mathrm{~K}-455 \mathrm{~K}$ and for pressures $p \leqslant 190 \mathrm{MPa}$. As the equation of state of liquid $n$-heptane is well known over a wide $p, T$-range ${ }^{(388)}$ the effects of changing density and temperature on the molecular motions could be separated by measuring the spin relaxation as a function of pressure and temperature. As the RHS model provides a valid Oth order approximation to simple dense liquids and further is very sensitive to density changes Powles and Gough $^{(382)}$ considered density as the primary parameter in determining the dynamic structure of liquids. They presented a seminal discussion of the interpretation of the results for $n$-heptane in terms of various theories, much in the spirit of van der Hart's ${ }^{(349)}$ later investigation of chloroform. Selected experimental $T_{1}$-isotherms are shown in Fig. 62 . Only average proton- $T_{1}$ have been reported as the relaxation times of the methylene- and methyl groups turned out to be very similar under ambient conditions and resolution requirements could not be met under high pressure conditions. Contrary to theoretical expectations, but often observed in practice, an exponential decay of the magnetization was measured at temperatures $T<380 \mathrm{~K}$ at all pressures, and also at higher temperatures and high pressures $p>100 \mathrm{MPa}$. In the low pressure-high temperature region a pronounced non-exponential decay of the magnetization was observed.

Though magnetic dipole-dipole interactions certainly provide the dominant relaxation mechanism, the authors did not discuss in detail the corresponding relaxation equations. They were interested mainly in the dependence of $T_{1}$ on density $\rho$ and temperature $T$ through the integral reorientational correlation time $\tau_{2}(\rho, T)$ and the self-diffusion coefficient $D(\rho, T)$. Within an activated state analysis the activation parameters, $\Delta H^{*}=\Delta E^{*}+p \Delta V^{*}$, in eqn (109) were found to depend on temperature and pressure respectively as is immediately clear from the curved isotherms and isobars (Fig. 62). Hence the physical parameters of the activated state theory, $\Delta H^{*}$ and $\Delta V^{*}$, could not be deduced from the experimental slopes, which yielded

$$
\Delta H^{+}=\Delta H^{*}-T\left(\frac{\partial \Delta H^{*}}{\partial T}\right)_{p},
$$

and

$$
\Delta V^{+}=\Delta V^{*}+p\left(\partial \Delta V^{*} / \partial p\right)_{\mathbf{T}}
$$

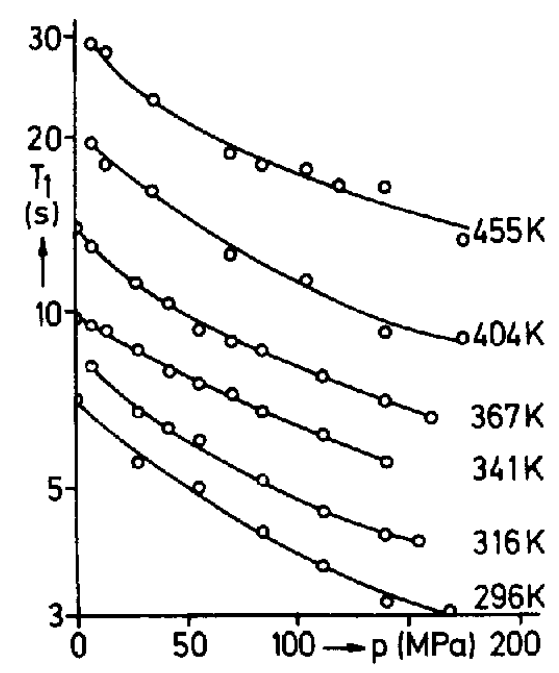

FIG. 62. Pressure dependence of selected ${ }^{1} \mathrm{H}-T_{1}$ isotherms in liquid $n$-heptane. ${ }^{(382)}$ 
because the dependence of $\Delta H^{*}(T)$ and $\Delta V^{*}(p)$ could not be obtained from the experimental data. Simple free volume theories require:

$$
\ln T_{1} \sim V_{\mathrm{o}} /\left(V-V_{\mathrm{o}}\right)
$$

and $T_{1}$ to be independent of temperature at constant density, which is clearly not observed in Fig. 63 . Hence for the free volume model to hold $V_{\mathrm{o}}$ must depend on $p$ and $T$, and one has to determine $\left(\partial V_{\mathrm{o}} / \partial T\right)$ and $\left(\partial V_{\mathrm{o}} / \partial p\right)$ also from the experimental data, which seems impossible. Powels and Gough $^{(382)}$ suggested the $p, T$-dependence of $V_{0}$ might be expected to correspond to the typical volume changes in glasses. In summary, one might expect a $p, T$-dependence of $V_{0}$, as well as for the activation parameters $\Delta H^{*}$ and $\Delta V^{*}$ irrespective of their precise physical meaning, but these dependences, as well as the parameters themselves, cannot be deduced readily from the $p, T$-dependence of relaxation rates. Furthermore both models do not seem to reflect all aspects of molecular motions when studied over wide ranges of density and temperature.

Nolle and Mahendroo ${ }^{(350)}$ were among the first to perform HP-NMR relaxation time studies of benzene, toluene and cyclohexane. Though the samples were purified and degassed, residual dissolved oxygen render their proton $T_{1}$-values less reliable. Brooks et al. ${ }^{(389)}$ showed also in their study of the pressure dependence of $T_{1}$ in benzene that, contrary to the findings of Nolle and Mahendroo, ${ }^{(350)}$ the proton $T_{1}$ vary less rapidly with pressure than the fluidity, $\eta^{-1}$. It must be emphasized, however, that no separation of intra- and intermolecular contributions to the total dipolar relaxation rate has been given and that only the intermolecular relaxation rate is expected from hydrodynamic considerations to vary in proportion to $D \sim \eta / T$. Indeed, self diffusion $D$ and viscosity $\eta$ showed a similar pressure dependence with $\Delta V^{*}(D)=20 \mathrm{~cm}^{3} / \mathrm{mol}^{(390)}$ and $\Delta V^{*}(\eta)=20.5 \mathrm{~cm}^{3} / \mathrm{mol}^{(391)}$ whereas the corresponding pressure coefficient of the total proton- $T_{1}$ has been found to be $\Delta V^{*}\left({ }^{1} H-T_{1}\right)$ $=16.3 \pm 0.2 \mathrm{~cm}^{3} / \mathrm{mol}^{(389)}$ Woessner and Snowden ${ }^{(392)}$ measured the pressure dependence of the deuteron spin-lattice relaxation in perdeuterobenzene at $300 \mathrm{~K}$ up to $p \simeq 40 \mathrm{MPa}$ and obtained $\Delta V^{*}\left({ }^{2} \mathrm{H}-T_{1}\right)=7.39 \pm 0.44 \mathrm{~cm}^{3} / \mathrm{mol}$. As the benzene molecule possesses a high reorientational mobility around the sixfold symmetry axis even in the solid state, ${ }^{(393)}$ this latter motion is obviously much less affected by pressure than are translational motions. Bull and Jonas ${ }^{(\mathbf{3 9 4})}$ were able to separate intra- and intermolecular contributions to the dipolar spin relaxation in benzene and chlorobenzene by the isotopic dilution technique ${ }^{(395)}$ and thereby determined the effect of pressure on reorientational $\left(\sim R_{1, \text { intra }}\right)$ and translational $\left(\sim R_{1}\right.$, inter $)$ motions of these molecules in the liquid state. In both liquids the effect of pressure on the normalized intermolecular dipolar relaxation times $\left(T_{1}(p) / T_{1}\left(p_{0}\right)\right)_{\text {inter }}$ is identical to the normalized viscosity $\left(\eta\left(p_{0}\right) / \eta(p)\right)\left(p_{0}=0.01 \mathrm{MPa}\right)$, corroborating the essentially diffusive character of the underlying translational motions (eqns (21 and 49)).

The intramolecular motions, which are only reorientational, were much less affected. This difference is especially pronounced in liquid benzene where the integral orientational correlation time is determined mainly by the fast anisotropic reorientation about the $\mathrm{C}_{6}$-axis (Fig. 64). The latter is only weakly affected by increasing density and is thus largely decoupled from translational motions. This

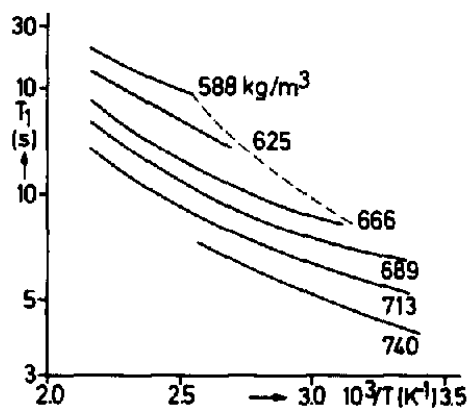

FIG. 63. Isobaric temperature dependence of proton relaxation times $T_{1}$ in $n$-heptane. (382) 


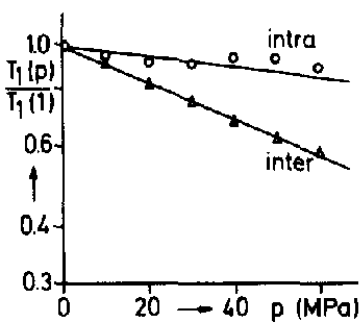

FIG. 64. Pressure dependence of intra- and intermolecular dipolar relaxation times $T_{1}$ in liquid benzene. ${ }^{(394)}$

coupling is, however, strongly enhanced as the sixfold symmetry of the molecule is destroyed by chlorine substitution which also introduces a dipole moment to the benzene molecule. The corresponding pressure coefficients $\Delta V^{*}$ (intra) $=7.4 \mathrm{~cm}^{3} / \mathrm{mol}$, and $\Delta V^{*}$ (inter) $=22 \mathrm{~cm}^{3} / \mathrm{mol}$, of the rotational and translational motions in liquid benzene agree well with those obtained from deuterium relaxation times and self-diffusion and viscosity data. They differ by a factor of about three, as has been found also for liquid acetone, ${ }^{(380)}$ but are almost identical in chlorobenzene, where $\Delta V^{*}$ (inter) $=11.2 \mathrm{~cm}^{3} / \mathrm{mol}$ and $\Delta V^{*}$ (intra) $=11.7 \mathrm{~cm}^{3} / \mathrm{mol}$ (Fig. 65).

At the beginning of the 70 s, Jonas's group ${ }^{(396)}$ for the first time achieved a sufficient homogeneity of $B_{0}$ over the sample volume of the high pressure cell to obtain high resolution spectra. In addition to the effect of pressure on the ${ }^{19} \mathrm{~F}$ chemical shifts in benzotrifluorides ${ }^{(396,397)}$ deuterium spin-lattice relaxation times of the methyl- and ring-deuterons in toluene- $\mathrm{d}_{8}$ were measured at $298 \mathrm{~K}$ and $373 \mathrm{~K}$ over a pressure range of $p \leqslant 300 \mathrm{MPa}$ (Fig. 66a). ${ }^{(396)}$ Spin relaxation of the ring-deuterons provides an estimate of the overall tumbling correlation time $\tau_{2}$ whereas the relaxation of the methyl-deuterons contains contributions from overall tumbling motions as well as fast spinning motions around the $\mathrm{C}_{3}$-symmetry axis of the $\mathrm{CD}_{3}$ group. Increasing pressure was seen to affect the overall tumbling motion much more than the internal rotation (Fig. 66b) as is reflected in the slope parameter $\Delta V^{*}=7.7 \mathrm{~cm}^{3} / \mathrm{mol}$ and $\Delta V^{*}=2.0 \mathrm{~cm}^{3} / \mathrm{mol}$ at high temperature $(T=373 \mathrm{~K})$. Jonas and coworkers further investigated a series of monosubstituted benzenes with HP-NMR. The main interest in these studies was a determination of the separate pressure effects on intra- and intermolecular dipolar relaxation times, hence on rotational and translational motions, and an investigation of the influence of molecular symmetry and dipole moments onto the coupling between both modes in liquids. Along these lines Parkhurst et al. ${ }^{(398)}$ measured at $T=303 \mathrm{~K}$ the pressure dependence of the proton spin-lattice relaxation times in liquid bromobenzene up to $p=250 \mathrm{MPa}$, and in toluene- $d_{3}$ up to $p=450 \mathrm{MPa}$. The separation of inter- and intramolecular dipolar contributions to the proton- $T_{1}$ was achieved by the isotopic dilution technique. Both molecules possess a very similar molecular shape as the van der Waals volumes of the substituent groups, $\mathrm{CH}_{3}: V=13.7 \mathrm{~cm}^{3} / \mathrm{mol} ; \mathrm{Br}$ : $V=14.4 \mathrm{~cm}^{3} / \mathrm{mol}^{(399)}$ are very similar, but their dipole moments differ considerably (toluene $\mu=0.4 \mathrm{D}$, bromobenzene $\mu=1.7 \mathrm{D}$ ). Again the change with pressure of the intermolecular contributions $R_{1}$, inter follows the behaviour predicted by hydrodynamic theory. The interesting part, however, is a comparison of the intramolecular dipolar relaxation rates in both liquids. As a qualitative measure of rotation-translation coupling Parkhurst et al. ${ }^{(398)}$ proposed the difference in pressure coefficients $\Delta V^{*}$ between viscosity $\left(\Delta V^{*}(\eta) \simeq \Delta V^{*}\right.$ (trans)) and intramolecular relaxation rate $\left(\Delta V^{*}\left(R_{1, \text { intra }}\right) \simeq \Delta V^{*}(\right.$ rot $\left.)\right)$ data of both liquids. Contrary to benzene ${ }^{(394)}$ the difference is small, as found for chlorobenzene, and is almost equal in both liquids, suggesting that molecular shape rather than the dipole moment is the determining factor as regards rotation-translation coupling in molecular liquids.

In a subsequent study, Assink et al. ${ }^{(400)}$ measured the pressure dependence $(p \leqslant 400 \mathrm{MPa}$ ) at $303 \mathrm{~K}$ of the deuteron spin-lattice relaxation time of fully deuterated and monodeuterated pyridine, toluene, fluorobenzene, chlorobenzene, bromobenzene and benzylcyanide. Proton relaxation times were also measured under the same conditions in fluorobenzene and pyridine, and deuterium relaxation times 


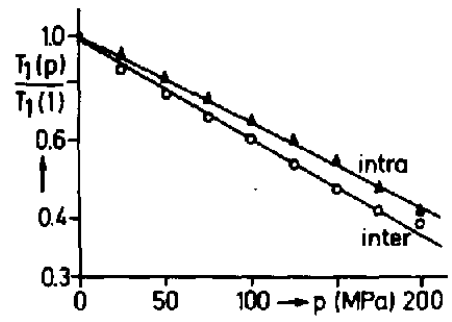

FIG. 65. Pressure dependence of intra- and intermolecular dipolar relaxation times $T_{1}$ in liquid chlorobenzene. ${ }^{(394)}$
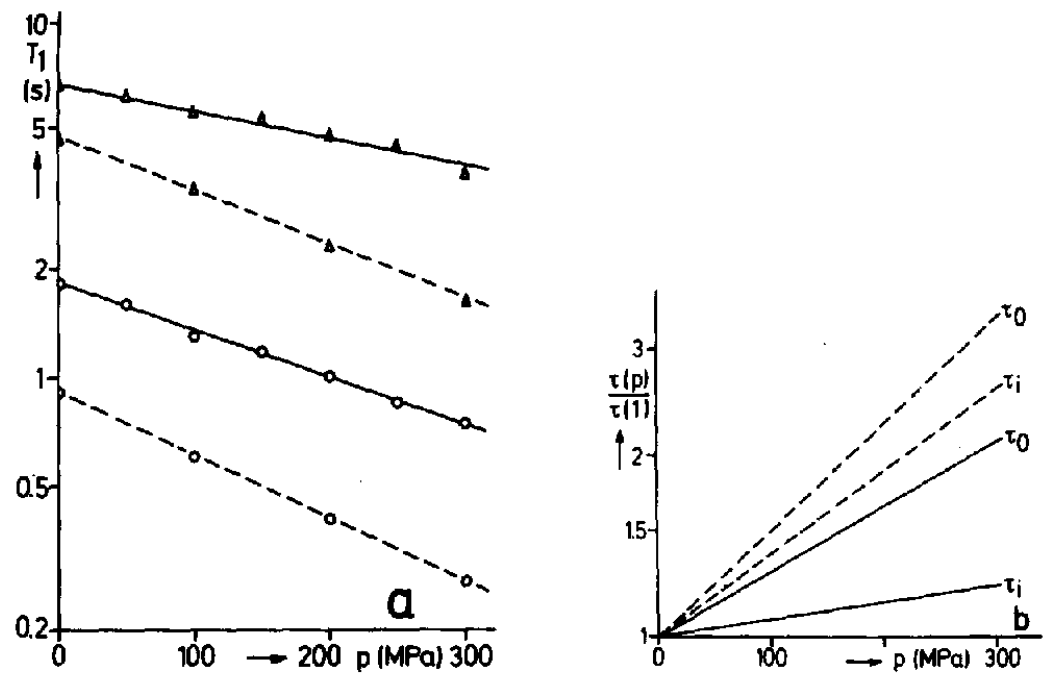

FIG. 66. (a) Pressure dependence of the ${ }^{2} \mathrm{H}-T_{1}$ of the ring $(O)$ and methyl- $\mathrm{d}_{3}(\Delta)$ deuterons in toluene- $\mathrm{d}_{8}$ at $373 \mathrm{~K}$ $(-)$ and $298 \mathrm{~K}(-)^{(396)}$ (b) Pressure dependence of normalized overall $\tau_{0}$ and internal $\tau_{1}$ correlation times of toluene- $d_{8}$ at $373 \mathrm{~K}(-)$ and $298 \mathrm{~K}(-)^{(396)}$

in fully deuterated nitrobenzene. A comparison of the fully deuterated and the monodeuterated compounds provides an estimate of the anisotropy of the rotational diffusion process in these liquids. As in the monodeuterated molecules, the deuteron has been substituted in a position adjacent to the other substituent. The corresponding deuterium relaxation rates monitor essentially the reorientation of the dipole moment vector of these benzene derivatives. Because the $\mathrm{QCC}$ is not expected to depend significantly on the substituent, an average value $\chi=180 \mathrm{kHz}$ was used in eqn (60) to calculate integral orientational correlation times $\tau_{2}$ as a function of pressure.

These data have been discussed, together with viscosity data obtained under the same conditions, in terms of the rotation-translation coupling parameter $\kappa$. The latter provides information about the anisotropy of the intermolecular potential, with $\kappa \simeq 1$ for strongly directional intermolecular forces, and $\kappa \simeq 0$ in the case of hard sphere interactions. According to Kivelson et al., ${ }^{(401)} \kappa$ gives the ratio of the intermolecular torques on the solute molecules to the intermolecular forces on the solvent molecules, and only the anisotropic part of the potential gives rise to the torques. The molecular radii in eqn (22) were determined from molar volumes, assuming hexagonal close-packed hard spheres. While no correlation was found between the dipole moments $\mu$ of the molecules and the RT-coupling parameter $\kappa$, the latter was found to be strongly correlated with molecular shape, as is exemplified in 
Fig. 67. Intermolecular interactions in these liquids are only slightly anisotropic causing a weak RT-coupling $(0.01<\kappa<0.2)$. Hence, orientational fluctuations exert only a small effect upon the dynamic structure in these liquids. Increasing density results in a decrease of $\kappa$, as seen in Fig. 68, because intermolecular torques increase much slower than intermolecular forces. These results led Assink et al ${ }^{(400)}$ to suggest that for $\kappa \gtrsim 0.4$ any pressure dependence is very small or totally absent. Another approximate measure of RT-coupling is provided by the difference of the experimental pressure coefficients $\delta \Delta V^{*}=\Delta V^{\#}(\eta)-\Delta V^{*}\left(R_{1 Q}\right)$. A large difference $\delta \Delta V^{*}$ corresponds to a weak RT-coupling, as in benzene, and vice versa. Again a distinct correlation between a large volume of the substituent and a strong RT-coupling is observed, whereas no obvious correlation with the respective dipole moments has been found.

Information about possible anisotropic reorientations of the various monosubstituted benzenes may be obtained from a comparison of deuteron relaxation times of the monodeuterated and fully deuterated molecules. The ratio of the respective parameters $\kappa$ is expected to reflect this anisotropy as has been demonstrated by an approximate correlation with the inertial anisotropy of the molecules. Within the series of monosubstituted benzenes discussed above, the substituent groups in toluene $\left(-\mathrm{CH}_{3}\right)$ and benzyl cyanide $\left(-\mathrm{CH}_{2} \mathrm{C} \equiv \mathrm{N}\right)$ provide an internal rotational degree of freedom to these otherwise rigid molecules.

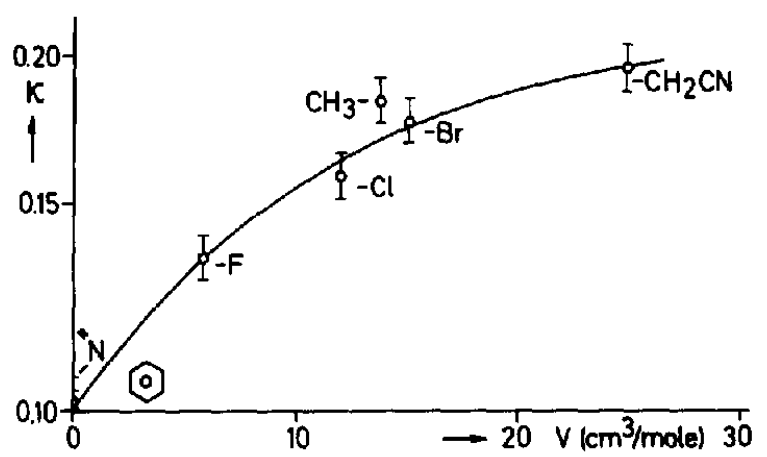

FlG. 67. Dependence of the rotation-translation coupling parameter $\kappa$ upon the volume of the substituent in monosubstituted benzenes. ${ }^{(400)}$

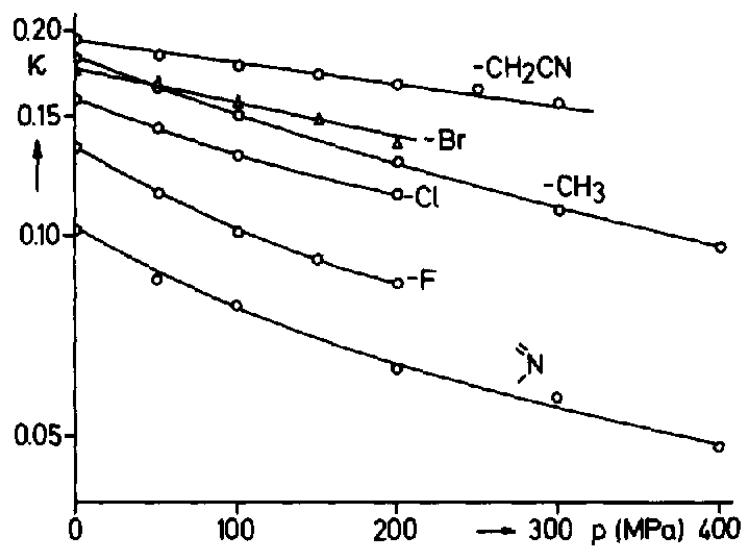

FIG. 68. Pressure dependence of $\kappa$ for monosubstituted benzenes. ${ }^{(400)}$ 
Therefore De Zwaan and Jonas, ${ }^{(402)}$ and Wilbur and Jonas, ${ }^{(403)}$ further investigated the effects of pressure on the overall and internal rotation in these liquids with deuterium HP-NMR. De Zwaan and Jonas ${ }^{(\mathbf{4 0 2 )}}$ measured the pressure dependence of the deuteron spin-lattice relaxation times in two selectively deuterated benzyl cyanides, benzyl-4- $d_{1}$ cyanide, and $\alpha, \alpha$-dideuterio-benzyl cyanide at $303 \mathrm{~K}$ and $423 \mathrm{~K}$ and at pressures up to $p \leqslant 300 \mathrm{MPa}$. The ${ }^{2} \mathrm{H}-R_{1 \mathrm{Q}}$ in the former compound largely reflected the overall tumbling of the molecule, whereas the spin relaxation of the latter monitored both the overall tumbling motion as well as the internal rotation of the substituent group. It was shown earlier ${ }^{(\mathbf{4 0 4})}$ that both reorientations proceed at comparable rates, hence a rotational diffusion model can be used to describe both motions. Overall rotational diffusion coefficients $D_{\text {rol }}=\left(6 \tau_{2}\right)^{-1}$ deduced from ring deuteron relaxation rates, assuming a $\mathrm{QCC}=180 \mathrm{kHz}$, were found in fair agreement with predictions from a hydrodynamic model (eqn (22)) modified with the microviscosity factor $f_{\mathrm{r}}$ as given by Gierer and Wirtz. ${ }^{(405)}$ The corresponding pressure coefficients $\Delta V^{*}\left(D_{\text {rot }}\right)$ and $\Delta V^{*}(\eta)$ respectively were determined to be very close, and this suggests a strong RT-coupling in liquid benzyl cyanide. There exists, however, a discrepancy between both measures of the strength of RT-coupling, the difference in apparent activation volumes $\delta \Delta V^{*}$ for rotation and translation and the Kivelsonparameter $\kappa$. The latter was found by Assink et al. to lie in the range $0.15 \leqslant \kappa \leqslant 0.2$ for benzyl cyanide, suggesting a weak RT-coupling. This casts further doubt on any physical interpretation of the slope parameter $\Delta V^{*}$ within the realm of an activated state picture. At least both results justified the use of the small-step diffusion model to represent the overall tumbling motion. The internal rotation of the $-\mathrm{CD}_{2} \mathrm{C} \equiv \mathrm{N}$ group has been described within an independent two-mode approximation (overall tumbling mode $D_{\text {rot }}$, diffusion of the substituent group around the symmetry axis of the methylcyanide group $D_{\text {int }}$ ), and within a symmetric top approximation with a symmetry axis through the $C_{1}$ and $C_{4}$ ring carbon atoms. Both approaches yield slightly different results which, however, show that the internal motion is certainly in the diffusion limit at $303 \mathrm{~K}$, especially at high pressure. However, inertial effects must be taken into account at $T=423 \mathrm{~K}$ over the whole pressure range as judged by the $\chi$-test ${ }^{(346)}$ and by the rather weak pressure dependence of $D_{\text {int }}$ at high temperature. The latter aspect has been considered further by Wilbur and Jonas ${ }^{(403)}$ in their HP-NMR study of liquid toluene- $\mathrm{d}_{8}$. The spin-lattice relaxation of both ring and methyl deuterons has been measured over the temperature range $238 \mathrm{~K} \leqslant T \leqslant 473 \mathrm{~K}$ covering a pressure range $p \leqslant 400 \mathrm{MPa}$. Viscosities and densities were also measured over this $p, T$-range. Liquid toluene- $\mathrm{d}_{8}$ has also been studied under high pressure conditions by Wilbur and Jonas ${ }^{(\mathbf{3 9 6})}$ and Parkhurst et al. ${ }^{(398)}$ who determined the proton relaxation time $T_{1}$ at $303 \mathrm{~K}$ and pressures $p \leqslant 300 \mathrm{MPa}$. Several authors measured the temperature dependence of the deuteron- $T_{1}$ in toluene- $\mathrm{d}_{8},{ }^{(392)}$ the ${ }^{1} \mathrm{H}-,{ }^{2} \mathrm{H}$ - and ${ }^{13} \mathrm{C}-T_{1}$ in protonated and deuterated toluene $\mathrm{e}^{(\mathbf{4 0 6 )}}$ and the proton and deuteron relaxation in toluene and toluene- $\mathrm{d}_{\mathrm{8}} \cdot{ }^{(407)} \mathrm{O}$ 'Reilly and Peterson ${ }^{(408)}$ have measured the self-diffusion coefficient $D(T)$ under ambient pressure conditions as well.

In order to extract integral orientational correlation times $\tau_{2}$ from the experimental deuterium relaxation rates, quadrupole couping constants $\chi=179.9 \mathrm{kHz}(\eta=0.056)^{(409)}$ and $\chi=165 \mathrm{kHz}^{(410)}$ appropriate to the ring and methyl deuterons of solid toluene respectively have been used. The overall tumbling motion of the toluene- $d_{8}$ molecule has been discussed in terms of the rotational diffusion model, the extended $J$-diffusion model and the corrected Enskog theory. The internal rotation of the methyl- $d_{3}$ group has been discussed further in terms of rotational diffusion, $120^{\circ}$ angle jump diffusion and the Langevin model.

A comparison of the overall tumbling correlation times $\tau_{2}$ with the modified Debye-StokesEinstein relation (eqn (119)) quickly makes it obvious that the tumbling motion of toluene is not well represented by this first order hydrodynamic diffusion model. Most of the disagreement is due to the different temperature and pressure dependences of $\tau_{2}$ and $(\eta / T)$. In addition, the RT-coupling parameter $\kappa=0.1$ is small indicating that orientational and positional fluctuations are largely decoupled in liquid toluene. Application of the $\chi$-test further indicates that the tumbling motion is in the diffusion limit at low temperatures and high pressures only. A comparison of isobaric and isochoric temperature dependences of the tumbling correlation time (Fig. 69) shows that the slowing down of reorientational motions at constant pressure is due to a reduction in thermal energy, as much as it is due to a reduction in density. Hence constant density data are necessary to separate clearly the 

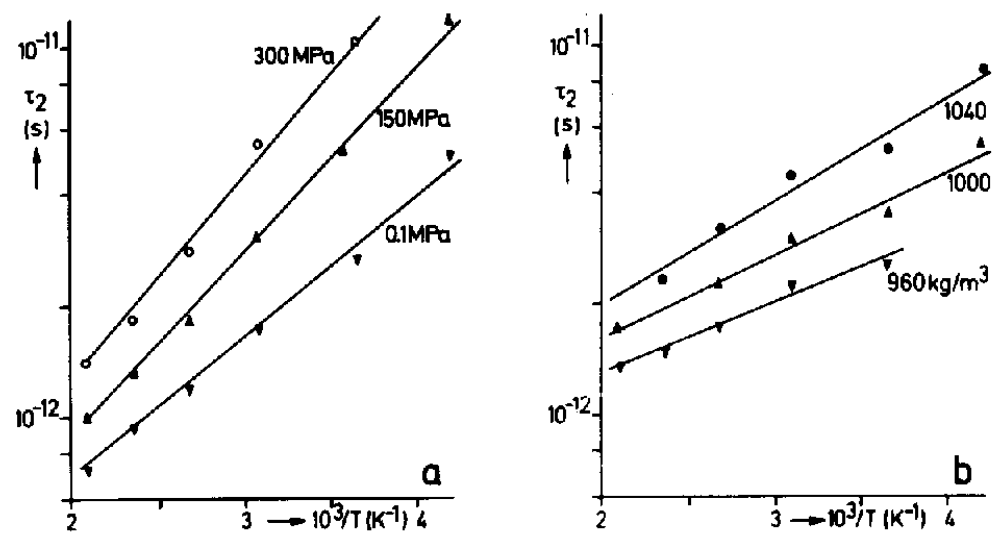

FIG. 69. Isobaric (a) and isochoric (b) temperature dependence of rotational correlation times $\tau_{2}$ in toluene- $d_{8} \cdot{ }^{(403)}$

effects of thermal energy and volume upon the molecular reorientation processes. As the rotational diffusion model has been shown not to represent the reorientation process properly the authors assumed the validity of the extended $J$-diffusion model to calculate the angular momentum correlation times $\tau_{\mathrm{j}}$ from the corresponding orientational correlation times. Using a procedure proposed by Gordon et al. ${ }^{(41)}$ they obtained hard sphere diameters at each temperature and pressure and then calculated the Enskog correlation time $\tau_{\mathrm{E}}$ with eqn (73). Using the relation between $\tau_{\text {, and }} \tau_{\mathrm{E}}$, Wilbur and Jonas ${ }^{(403)}$ then recalculated the corresponding relaxation times and observed good agreement between calculated and experimental data.

However, the argument is somewhat circular, as the calculations have been forced to agree with experiment. This has been recognized by the authors, however, when they stated that these results were only qualitative. Applying the rotational diffusion model with isotropic overall and anisotropic internal rotation, either small-step or $120^{\circ}$ angle jump, to the methyl deuteron $T_{1 Q}$, Wilbur and Jonas concluded, not unexpectedly, that both models are not well suited to describe the internal rotation of the methyl group and that the internal reorientation is very fast in the whole $p, T$-range investigated. However, application of the rotational Langevin model shows that the internal rotation is also not close to the free rotor limit. Hence conclusions drawn from the application of the diffusion models and the Langevin model in the large friction limit, where it becomes equivalent to the diffusion model, have at least qualitative significance. From the isochoric temperature dependence of the internal correlation time $\tau_{\text {int }}$ it is obvious that its isobaric temperature dependence is dominated by energy effects. Internal reorientations of methyl groups are therefore much less affected by density changes than overall reorientations. This conclusion is also supported by the high internal mobility found for methyl groups in many molecular crystals.

The major drawback in the study of benzyl cyanide is the impossibility of determining angular

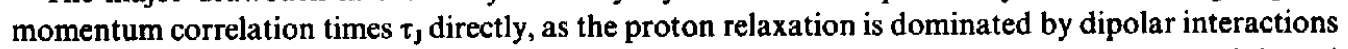
with spin-rotation interactions being negligible. This shortcoming was overcome by Assink and Jonas ${ }^{(412)}$ and De Zwaan et al. ${ }^{(413)}$ who studied the spin-relaxation of the deuterium $\left({ }^{2} \mathbf{H}\right)$ and fluorine $\left({ }^{19} \mathrm{~F}\right)$ nuclei of fluorobenzene- $d_{5}$ over a temperature range from $303 \mathrm{~K}$ to $623 \mathrm{~K}$ and over a pressure range $p \leqslant 350 \mathrm{MPa}$ in the liquid and dense fluid phase. Assink $e$ al $^{(\mathbf{4 1 2 )}}$ also reported deuterium spin-lattice relaxation measurements at $303 \mathrm{~K}$ and $p \leqslant 200 \mathrm{MPa}$ in liquid fluorobenzene$d_{1}$ and fluorobenzene- $d_{5}$ as discussed already above. Green and Powles ${ }^{(414)}$ have also reported the temperature dependence of the proton $\left({ }^{1} \mathrm{H}\right)$ and fluorine $\left({ }^{19} \mathrm{~F}\right)$ spin-lattice relaxation times at ambient pressure.

Quadrupole interactions provide the sole relaxation mechanism of the deuterons and yield integral orientational correlation times $\tau_{2}$. Spin-rotation interactions dominate the relaxation of the fluorine nuclei and yield angular momentum correlation times $\tau_{\mathrm{J}}$. A QCC of $\chi=180 \mathrm{kHz}$ has been assumed in 
accord with similar coupling constants determined in other monosubstituted benzenes and the finding that different substituents do not change the QCC much. Spin-rotation coupling constants were calculated from the diagonal components of the spin-rotation interaction tensor $c$ as determined by molecular beam measurements, ${ }^{(415)}$ neglecting any anisotropic components. Small corrections to the total ${ }^{19} \mathrm{~F}-R_{1}$ were made because of contributions from dipolar interactions and anisotropic chemical shielding interactions. The resulting orientational $\left(\tau_{2}\right)$ and angular momentum $\left(\tau_{\mathrm{J}}\right)$ correlation times were made to agree with predictions from the extended $J$-diffusion model appropriate for spherical top molecules if the spin-rotation coupling constant $C_{\text {eff }}$ (eqn (118)) was adjusted slightly (Fig. 70). This was necessary because the authors originally used an effective spin-rotation constant

$$
C_{\mathrm{eff}}^{2}=\frac{1}{3} \cdot \sum_{\mathrm{i}=\mathrm{a}, \mathrm{b}, \mathrm{c}} C_{\mathrm{ii}}^{2}=(3.12 \mathrm{kHz})^{2},
$$

with $C_{\mathrm{ii}}$ given in the principal frame of the inertia tensor instead of $C_{\text {eff }}^{2}=C_{0}^{2}+2 \Delta C^{2}\left(\tau_{\mathrm{sR}} / \tau_{\mathrm{J}}\right)$ with $C_{\|}$ and $C_{\perp}$ given in the main axis system of the spin-rotation interaction tensor. Both quantities become identical only if the diffusion limit $\left(\tau_{\mathrm{J}} \leqslant \tau_{2}\right.$ and $\left.\tau_{\mathrm{J}}=\tau_{\mathrm{sa}}\right)$ holds, and if the principal frames of the inertia tensor and the spin-rotation tensor coincide. A general limitation is the spherical top approximation, which, however, has often to be made. The best fit coupling constant $C_{\text {eff }}=3.60 \mathrm{kHz}$ is in good agreement with $C_{\text {eff }}=3.32 \mathrm{kHz}$ which follows from the results of Green and Powles. ${ }^{(414)}$

De Zwaan et al. ${ }^{(413)}$ also determined the density of fluorobenzene over the whole $p, T$-range and were thus able to separate the effects of density and temperature on the correlation times $\tau_{3}$ and $\tau_{2}$, and hence on the molecular reorientations. An interesting observation in the dense fluid phase was the near constancy over a large density range and constant thermal energy of the integral orientational correlation time $\tau_{2} \sim R_{1}$, accompanied by a strong decrease of the angular momentum correlation time $\tau_{3}$ with increasing density. Though the form of the orientational correlation function $G_{2}(t)$ certainly changes with density, the area under the curve does not. This finding indicates that integral correlation times derived from NMR relaxation rates in the fast motions limit $\left(\omega_{\mathrm{L}} \tau \ll 1\right)$ can only provide limited details of the orientational fluctuations in liquids. The strong decrease of $\tau$, with density reflects the increase in collision frequency with density, of course. De Zwaan et al. ${ }^{(413)}$ further considered the cell model and the Enskog theory in their ability to predict the density and temperature dependence of $\tau_{\mathrm{j}} \sim \tau_{\mathrm{coll}}$ (eqn (113)). The corresponding mean times between collisions $\tau_{\mathrm{coll}}$ are given by eqn (112). Both theories predict a $T^{-1 / 2}$ variation of $\tau_{\mathrm{s}}(T)$ at constant density which is contrary to what is generally observed in HP-NMR experiments. If, however, the hard sphere diameter $a$ is assumed to decrease with increasing thermal energy at constant density, agreement with the experimentally observed increase of $\tau_{\mathrm{J}}$ with $T$ could be reached. A similar variation of $\sigma(T)$ has been deduced by Wilhelm ${ }^{(416)}$ from gas solubility data and is reported generally from high-pressure self-diffusion coefficient measurements. The isothermal density dependence of $\tau_{1}(\rho)$ has been found to agree qualitatively with the cell model at low temperatures and the Enskog theory at supercritical

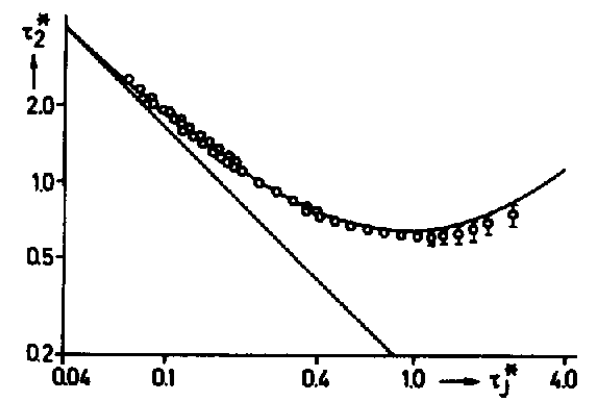

FIG. 70. Comparison of reduced orientational $\left(\tau_{2}^{*}\right)$ and angular momentum $\left(\tau_{f}^{*}\right)$ correlation times in fluorobenzene- $d_{5}$. The straight line corresponds to the Hubbard relation, eqn $(71)^{(413)}$ 
temperatures. At low densities, however, hard sphere approximations are expected to be less appropriate because of the increasing importance of attractive interactions. As regards orientational correlation times an Arrhenius dependence of $T_{1}(T)$ has been observed with an apparent activation enthalpy $\Delta H^{*}$ of the order of the mean thermal energy. Furthermore, $\Delta H^{*}$ increases with increasing pressure or increasing density. Both results again demonstrate that the activated state model is not appropriate.

6.2.3. Complex Viscous Liquids. With the exception of spin-rotation interactions, most other spin relaxation mechanisms are connected with spectral density functions of orientational and translational fluctuations. In the fast motion regime all the information obtainable is limited to integral correlation times characterizing overall and internal molecular motions. Density variations at constant thermal energy generally slow down translational and overall rotational diffusive processes much stronger than internal motions, hence provide a valuable means of separating these processes and enhance the requirements any motional model must meet in order to describe liquid state dynamics consistently. The situation can be further improved in highly viscous, often supercooled, liquids where molecular motions can be slowed down into the slow motions regime $\left(\omega_{\mathrm{L}} \tau \geqslant 1\right)$. The relaxation then depends on the explicit form of the spectral density functions and not just on the integral over the corresponding time correlation function. This poses further stringent constraints on any motional model used to interpret spin relaxation experiments. In viscous liquids, however, the relaxation process is no longer dominated by simple collisions as in non-viscous liquids but rather by complex cooperative reorientations. Therefore drastic simplifications have often to be made to interpret relaxation rate curves.

Artaki and Jonas, ${ }^{(417)}$ extending an earlier ambient pressure proton and deuteron relaxation study, ${ }^{(418)}$ investigated the dynamic structure of isopropyl benzene in the supercooled state. The proton, deuterium and carbon-13 relaxation times were measured in selectively deuterated isopropyl benzene (IPB) over the temperature range $125 \mathrm{~K}$ to $200 \mathrm{~K}$ and the pressure range $p \leqslant 500 \mathrm{MPa}$.

Besides exploring the origin of a discontinuity observed at a temperature $T_{\mathrm{K}}>T_{\mathrm{g}}$ in the temperature dependence of the shear viscosity of several glass forming liquids, ${ }^{(419)}$ the main objectives of their HP-NMR study were to test the validity of the modified Debye equation (eqn (119)) over a wide $p$, $T$-range at high viscosities and to detect and examine with NMR the $\alpha$ and $\beta$ relaxation processes observed in dielectric relaxation experiments. ${ }^{(\mathbf{4 2 0})}$ High pressure experiments are especially valuable in this respect as they allow the viscosity of supercooled liquids to be varied by many orders of magnitude. Though the discontinuity in the set of parameters in the VTF-equation (eqn (125)) used to represent $\eta(T)$ has been tentatively associated with a complete quenching of all rotational diffusive modes below $T_{\mathbf{K}},{ }^{(42)}$ Arndt and Jonas ${ }^{(418)}$ showed that isopropyl benzene molecules lose their translational mobility faster than their rotational mobility. To resolve this discrepancy Artaki and Jonas $^{(417)}$ investigated the very low frequency motions of supercooled isopropyl benzene (IPB- $\left.\mathrm{d}_{7}\right)$ via the rotating frame proton relaxation times $T_{1 \rho}\left({ }^{1} \mathrm{H}\right)$ of the ring protons as a function of isotopic dilution in perdeuterated IPB. For the sake of the argument, the authors approximately characterized the complicated rotational and translational diffusive motions of IPB molecules by effective single correlation times $\tau_{\text {rot }}$ and $\tau_{\text {trans, }}$, deduced from $T_{1}\left({ }^{1} \mathrm{H}\right)$ and $T_{1 \rho}\left({ }^{1} \mathrm{H}\right)$ data and plotted the former against the shear viscosity-temperature ratio ( $\eta / T$ ) (Fig. 71 ).

It was shown that a decisive discontinuity in the effective translational correlation times $\tau_{\text {trans }}$ at $T \simeq T_{\mathbf{K}}$ is present, indicating a sudden change in the nature of the translational motions in the neighbourhood of $T_{\mathbf{k}}$. But the modified Debye-equation is obeyed over almost four orders of magnitude with no change in the small RT-coupling parameter $\kappa \simeq 0.2$ at the temperature $T_{\mathrm{K}}$. The validity of the modified Debye-equation has been further studied by measuring the pressure dependence of the deuterium spin-lattice relaxation times of IPB- $\mathrm{d}_{5}$ in the fast motions regime at temperatures $200 \mathrm{~K} \leqslant T \leqslant 250 \mathrm{~K}$. The orientational correlation times $\tau_{2}$ show a non-linear dependence on $\eta / T$ indicating an increasing rotation-translation coupling with increasing density and an increased anisotropy of the molecular motions involved. In contrast to the effect of density changes isochoric plots of $\tau_{2}$ versus $\eta / T$ reflect the effect of kinetic energy changes on the RT-coupling parameter. At constant volume, $\tau_{2}$ depends linearly on $\eta / T$ suggesting that the coupling parameter $\kappa$ is not sensitive 


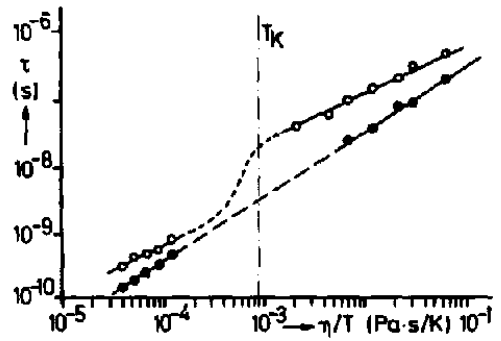

Fig. 71. Rotational $(O)$ and translational $(\Theta)$ correlation time $\tau$ versus the viscosity-temperature ratio $\eta / T$ in isopropylbenzene- $d_{7} .^{(417)}$

to changes in the kinetic energy of the molecules. In summary, Artaki and Jonas ${ }^{(417)}$ concluded that volume rather than kinetic effects determine the extent of the coupling between the rotational and translational motion of IPB- $\mathrm{d}_{\mathbf{s}}$.

Concerning a comparison of the proton $T_{1 \rho}$-data of the ring and isopropyl chain protons both relaxation rates show a maximum at about the temperature where the dielectric $\alpha$-relaxation peak is observed. This suggests that the overall tumbling motion is responsible for both relaxation maxima. A second relaxation rate maximum, though not observed, corresponds to the dielectric $\beta$-relaxation and is indicated by rising proton relaxation rates of the ring and chain protons respectively below the glass transition. These results may demonstrate that the $\beta$-relaxation peak has contributions from both rotational and translational motions of the isopropyl chain and the benzene ring.

The dependence of the RT-coupling parameter $\kappa$ on density, kinetic energy and molecular shape has been investigated further in a study of the ring deuteron spin-lattice relaxation rates of various supercooled, highly viscous liquids. ${ }^{(422)}$ As previous results indicated that molecular shape rather than permanent dipole moments determine the coupling between rotations and translations in molecular liquids, the variation of the former dictated the choice of the substituted benzenes studied. The compounds selected were isopropyl benzene- $d_{5}$, sec-butyl benzene- $d_{5}, n$-butyl benzene- $d_{5}$, toluene- $\mathrm{d}_{8}$, cis-decalin- $\mathrm{d}_{10}$ and sec-butyl cyclohexane- $\mathrm{d}_{5}$. For comparative purposes the substituted benzenes were selectively deuterated in the ring. All deuteron spin-lattice relaxation time measurements were performed in the fast motion regime $\left(\omega_{\mathrm{L}} \tau_{\mathrm{L}}<1\right)$, hence only integral orientational correlation times $\tau_{2}$ were obtained. Shear viscosities and molar volumes of these model compounds were also measured as a function of pressure and temperature. The results of the study show that asymmetric top molecules which tumble anisotropically exhibit a positive curvature of isothermal $\tau_{2}$ versus $(\eta / T)$ plots, corresponding to an increasing rotation-translation coupling with increasing density, as observed in isopropyl benzene- $d_{5}$ and sec-butyl benzene- $d_{9}$. As a qualitative physical explanation Artaki and Jonas ${ }^{(422)}$ suggest that as density increases, molecular reorientations become increasingly more hindered and anisotropic due to a closer proximity of the neighbouring molecules, and hence lead to enhanced rotation-translation coupling (Fig. 72). Symmetric top molecules in non-viscous liquids like toluene, cis-decalin and sec-butyl cyclohexane display a negative curvature in isothermal plots of $\tau_{2}$ versus $(\eta / T)$. These molecules possess at least one axis about which reorientations can proceed rather freely causing the RT-coupling to decrease with increasing density. The physical explanation given by the authors is that intermolecular torques do not increase as much as intermolecular forces with density which is tantamount to a negative density dependence of the RTcoupling parameter $\kappa$ implying a decoupling of these motional modes with increased density. Liquid $n$-butyl benzene shows a change from a negative to a positive curvature with increasing density.

Following the arguments given above the authors suggested that these molecules can perform rather unhindered reorientations about an axis of symmetry at lower densities and that the anisotropy in the overall tumbling motions increases with a closer packing of the molecules. Purely kinetic effects are best examined under constant density conditions. Linear $\tau_{2}$ versus $(\eta / T)$ isochors were obtained for all densities and all compounds measured. These constant slopes suggest that the coupling 

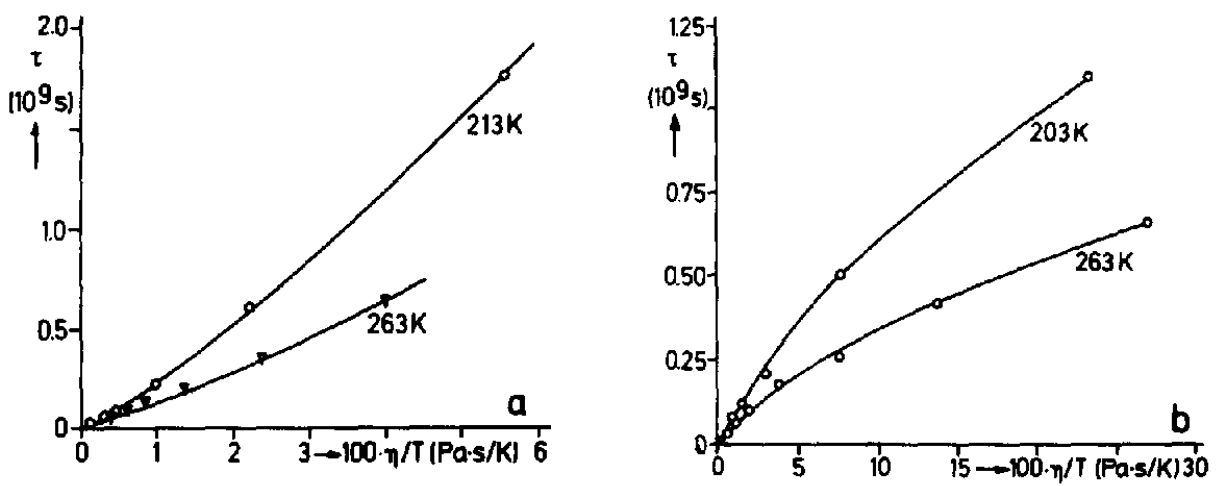

FIG. 72. Rotational correlation times $\tau_{2}$ versus $\eta / T$ for (a) sec-butylbenzene-d, and (b) sec-butylcyclohexane-dg. ${ }^{(422)}$

between rotational and translational modes is independent of the kinetic energy of the molecules. Therefore volume rather than kinetic energy determines the degree of RT-coupling. The authors pointed out, however, that the absolute values of the RT-coupling parameters are rather small $(\kappa \simeq 0.1-0.2)$ and that a description of strong RT-coupling in supercooled liquids should invoke coupling parameters $\kappa \lesssim 1$. Thus it seems questionable that the modified Debye-Stokes-Einstein equation can represent the dynamic behaviour of highly viscous supercooled liquids properly.

Two rather more complex molecules similar to the monosubstituted benzenes, 2-ethylhexyl benzoate (EHB) and 2-ethylhexyl cyclohexanecarboxylate (EHC), have been investigated by Jonas et al. ${ }^{(423)}$ and Adamy et al. ${ }^{(424)}$ with HP-NMR techniques. Natural abundance ${ }^{13} \mathrm{C}$ spin-lattice relaxation times and nuclear Overhauser enhancement (NOE) factors have been measured at temperatures $253 \mathrm{~K} \leqslant T \leqslant 353 \mathrm{~K}$ and pressures $p \leqslant 500 \mathrm{MPa}$. Both relaxation parameters are dominated by the intramolecular dipolar coupling of ${ }^{13} \mathrm{C}$ and directly bonded ${ }^{1} \mathrm{H}$ nuclear spins. Thus these relaxation coefficients monitor orientational fluctuations of the molecules as well as segmental motions of the alkyl chains and the ring flexibility in EHC. Self-diffusion coefficients, densities and shear viscosities have also been studied in both liquids. ${ }^{(425,426)}$ Jonas et al. ${ }^{(423,424)}$ followed the relaxation behaviour of each carbon nucleus in these molecules and were able to reproduce the relaxation time curves $T_{1}(T, p)$ by fitting the experimental isotherms to a Cole-Davidson distribution of orientational correlation times given by:

$$
p(\tau)=\begin{array}{cl}
{[\sin (\beta \pi) / \pi]\left(\tau / \tau_{m}-\tau\right)^{\beta}} & \text { for } 0 \leqslant \tau<\tau_{m} \\
0 & \text { for } \tau \geqslant \tau_{m}
\end{array}
$$

The authors pointed out that neither an isotropic diffusion model, nor an anisotropic model, or its mathematically equivalent two mode approximation (overall tumbling mode plus internal reorientation) represents the experimental relaxation curves properly. The isothermal pressure dependence was represented by an Arrhenius-type expression with the pre-exponential factor $\tau_{0}$ and the apparent activation volume $\Delta V^{*}$ assumed to be independent of pressure. The width parameter $\beta$ in eqn (119) is also independent of pressure as the absolute value of $T_{1}$ at the respective minima of the isotherms do not change with pressure. It is important to note that relaxation data in the slow motions regime are necessary for this purpose as $\beta$ cannot be determined uniquely from $T_{1}$ data in the fast motions regime. Representative isotherms of the ring (C5) carbons in both $\mathrm{EHB}$ and $\mathrm{EHC}$ are shown in Fig. 73. At low temperatures the EHC ring carbon relaxation time curves are much broader, reflecting the higher mobility and internal flexibility of EHC compared to EHB. This is a direct consequence of the conjugation of the carboxyl group and the phenyl ring in EHB rendering the molecule much more rigid. This is also reflected in the much smaller width parameters $\beta$ of the C5-ring carbons in EHC compared to EHB. In contrast, the EHB and EHC methane carbons show nearly identical motional characteristics with correspondingly similar $\beta$-parameters. These differences parallel trends observed 

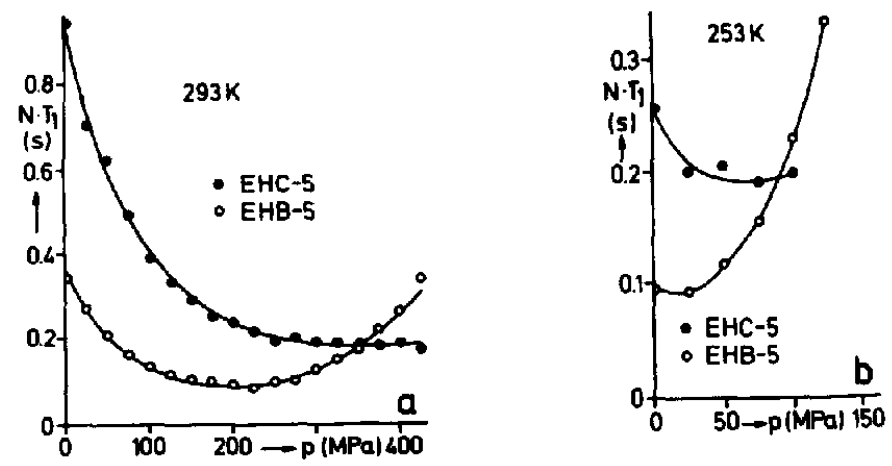

Fig. 73. Pressure dependence of the ${ }^{13} \mathrm{C}-T_{1}$ of the ring carbon (C5) in (a) 2-ethyl hexylbenzoate (EHB) and (b) 2-ethyl hexylcyclohexane carboxylate (EHC). ${ }^{(424)}$

in the fluidities and self-diffusion coefficients observed in both liquids at low temperatures and high pressures. As a consistency test cross-relaxation rates determining NOE-factors (eqn (120))

$$
N O E=1+\left(\gamma_{\mathrm{H}} / \gamma_{\mathrm{c}}\right) \frac{6 \mathrm{~g}_{2}\left(\omega_{\mathrm{H}}-\omega_{\mathrm{c}}\right)-\mathrm{g}_{\mathrm{o}}\left(\omega_{\mathrm{H}}-\omega_{\mathrm{C}}\right)}{g_{\mathrm{o}}\left(\omega_{\mathrm{H}}-\omega_{\mathrm{C}}\right)+3 g_{1}\left(\omega_{\mathrm{C}}\right)+6 g_{2}\left(\omega_{\mathrm{H}}+\omega_{\mathrm{C}}\right)}
$$

with

$$
\begin{aligned}
g_{m}(\omega) & =\int_{0}^{\infty} \frac{p(\tau) \tau}{1+(\omega \tau)^{2}} d \tau \\
& =\frac{\sin \left[\beta \arctan \left(\omega \tau_{\mathrm{m}}\right)\right]}{\omega\left[1+\left(\omega \tau_{\mathrm{m}}\right)^{2}\right]^{\beta / 2}}
\end{aligned}
$$

have been calculated using the CD-spectral density functions and optimized parameters deduced from the ${ }^{13} \mathrm{C}-T_{1}$ data. The good agreement observed, however, (Fig. 74) does not seem to provide a test of the validity of the model distribution chosen to represent the microscopic dynamics. Rather it provides a consistency test of eqn (45) and eqn (46) used to calculate the relaxation rates assuming that only nearest neighbour dipolar couplings dominate. The pressure coefficients of the average orientational correlation times $\langle\tau\rangle=\beta \tau_{\mathrm{m}}$ are rather similar in EHB and EHC at high temperatures (353 K) but differ substantially at low temperatures $(253 \mathrm{~K})$, indicating that the reorientation of the less
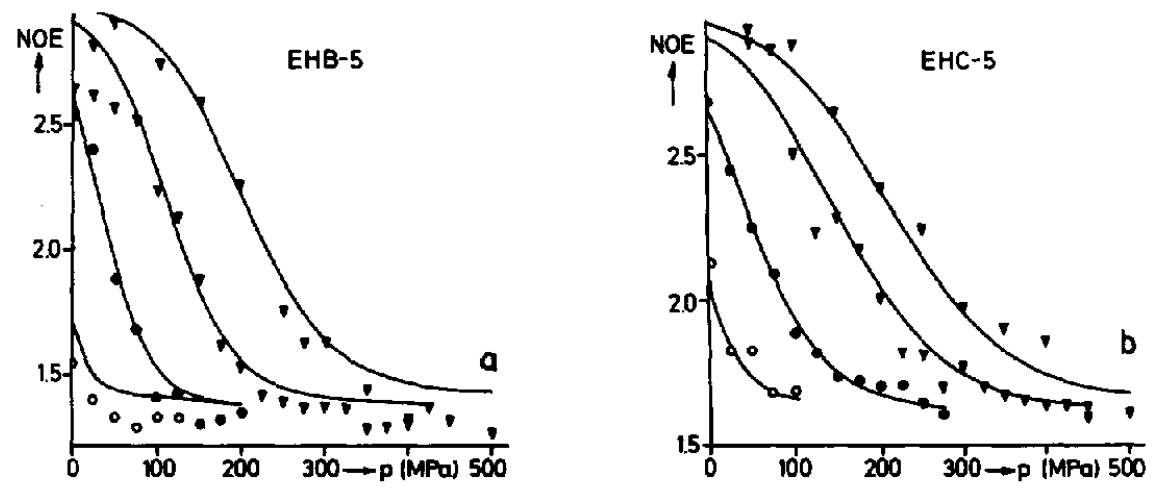

Fig. 74. Pressure dependence of the nuclear Overhauser enhancement (NOE) factors of the ring carbons (C5) in (a) EHB and (b) EHC at $(O) 313 \mathrm{~K},(0) 293 \mathrm{~K},(\nabla) 273 \mathrm{~K}$ and $(\nabla) 253 \mathrm{~K}$. $^{(424)}$ 
flexible EHB molecules are strongly hindered at low temperatures and high densities. The presence of the flexible cyclohexyl ring obviously allows the EHC molecule to change shape more easily than the EHB molecule to accommodate reorientation at higher densities.

\subsection{Hydrogen Bonded Liquids}

6.3.1. Alcohols. High pressure NMR relaxation investigations of liquid alcohols are surprisingly scarce despite the numerous investigations under ambient pressure conditions.

Fiorito and Meister ${ }^{(427)}$ reported HP-NMR measurements on three highly viscous associated liquids, glycerol, 1,3-butanediol and 2-methyl-2,4-pentanediol as a function of pressure ( $p \leqslant 300 \mathrm{MPa})$ and temperature $(250 \mathrm{~K}<T<340 \mathrm{~K})$. Proton spin-lattice $\left(T_{1}\right)$ and spin-spin $\left(T_{2}\right)$ relaxation rates were determined, with all $T_{1}$-data extending well into the slow motions regime $\left(\omega_{\mathbf{L}} \tau_{\mathbf{L}}>1\right)$. Due to difficulties with exchanging hydroxyl protons, inter- and intramolecular contributions to the average relaxation rates could not be determined. Therefore, Fiorito and Meister ${ }^{(427)}$ evaluated their data using the assumption that translational motions are the main source of motional modulation of the dipolar spin couplings. Later, Kintzinger and Zeidler ${ }^{(\mathbf{4 2 8})}$ demonstrated with an isotopic dilution study of glycerol- $\mathrm{d}_{3}$ in glycerol- $\mathrm{d}_{8}$ that the inter- and intramolecular dipolar contribution to the total relaxation rate are in fact comparable. In applying the translational diffusion theory of Torrey ${ }^{(106)}$ as corrected by Kubo and Tomita ${ }^{(429)}$ Fiorito and Meister adjusted the model parameters $\left(\tau_{r}, d\right)$ to the extensive temperature and frequency dependent NMR data of glycerol obtained under ambient pressure conditions. ${ }^{(430-432)}$ Self-diffusion coefficients calculated with these parameters are in good agreement with experimental values. ${ }^{(433,434)}$ At elevated pressures, translational correlation times $\tau_{\text {trans }}$ were obtained directly from the ratio $T_{1} / T_{2}$ in the dispersion region at each temperature and pressure.

The distance of closest approach $d$ was then determined unambiguously from the $T_{1}$ data. It was found to decrease with increasing pressure and decreasing temperature and to become smaller than a molecular diameter. The latter observation is inconsistent with the original formulation of the theory, and reflects the fact that the spin bearing nuclei do not reside in the center of the molecules as assumed. The scaled mean square jump length $\alpha=\left\langle r^{2}\right\rangle / 12 d^{2}$ has been found to be very small in glycerol $(\alpha \leqslant 0.005)$, and somewhat larger in 1,3-butanediol $(\alpha=0.07)$ and 2-methyl-2,4-pentanediol. All three liquids exhibit a pronounced non-Arrhenius temperature dependence of the isobaric translational correlation times $\tau_{\text {trans }}$ with the slopes being essentially independent of pressure (Fig. 75). The isothermal pressure dependence of $\ln \tau_{\text {trans }}$ is linear in glycerol with the slope increasing with decreasing temperature. In the other two liquids $\ln \tau_{\text {trans }}$ versus $p$ plots are distinctly non-linear, with a slope decreasing with increasing pressure. For glycerol, however, the slopes are independent of temperature. In addition, the diffusion coefficients calculated from the correlation times $\tau_{\text {trans }}$ (eqn (50)) show a pressure dependence different from the corresponding fluidity data contrary to what might be expected from simple hydrodynamic considerations (eqn (21)). It remains unclear, however, whether this failure is a consequence of the assumption that the total proton relaxation is due to translational motions only. However, these characteristics clearly demonstrate the inapplicability of activated jump models to the dynamics of viscoelastic liquids.

Wolfe and Jonas ${ }^{(435)}$ also investigated the pressure $(p \leqslant 500 \mathrm{MPa})$ and temperature $(263 \mathrm{~K} \leqslant T$ $\leqslant 400 \mathrm{~K})$ dependence of the deuteron spin-lattice $\left(T_{1}, T_{1 \rho}\right)$ and spin-spin $\left(T_{2}\right)$ relaxation in liquid glycerol- $\mathrm{d}_{4}\left(\mathrm{D}_{2} \mathrm{COH}\right)_{2} \mathrm{CHOH}$, and glycerol- $\mathrm{d}_{3}, \mathrm{C}_{3} \mathrm{H}_{5}(\mathrm{OD})_{3}$. Deuteron spin relaxation rates can be associated clearly with single particle correlation functions and their respective spectral densities. This is not the case with spin-1/2 nuclei, where intra- and intermolecular dipolar contributions have to be separated, often with grossly simplifying assumptions, and cross-relaxation effects (spin diffusion) complicate the association of a spin relaxation rate with a particular location on the molecule. The selective deuteration of glycerol enabled Wolfe and Jonas ${ }^{(435)}$ to monitor reorientational motions of backbone (glycerol- $d_{4}$ ) and hydroxyl (glycerol- $d_{3}$ ) deuterons. The authors tested three different motional models: single mode diffusion model, a two mode diffusion model, allowing for independent overall and internal reorientations, and a diffusion model with an asymmetric Cole-Davidson 


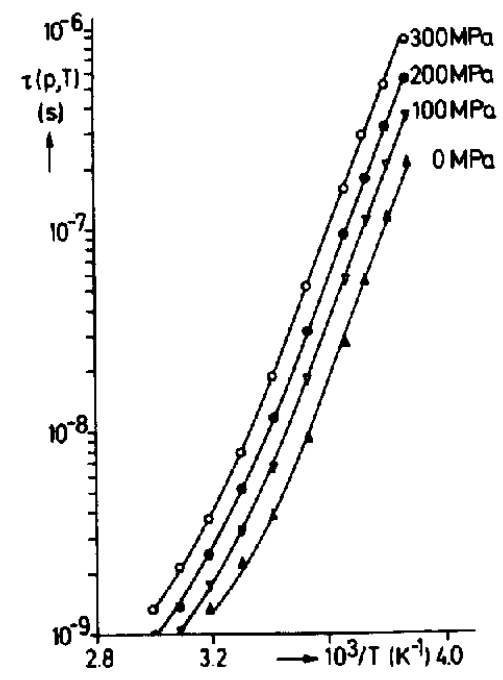

FIG. 75. Isobaric temperature dependence of translational correlation times $\tau(p, T)$ in glycerol. ${ }^{(427)}$

distribution of correlation times and concluded that only the distribution of correlation times model could be made to fit all $T_{1-}, T_{1 p^{-}}$and $T_{2}$-data consistently.

Carbon-13 relaxation rates ${ }^{(436)}$ can also be reproduced quantitatively but only qualitative agreement could be reached in predicting proton intramolecular relaxation rates at various frequencies. ${ }^{(428)}$ Large discrepancies have also been noted in comparisons with the deuteron $T_{1}$ and $T_{2}$ data of glycerol- $d_{8}$ reported by Drake and Meister. ${ }^{(437)}$ Deviations may have been caused by the use of water-contaminated glycerol samples by the latter investigators.

The deuteron spin-lattice relaxation time curves of glycerol- $\mathrm{d}_{4}$ and glycerol- $\mathrm{d}_{3}$ are mainly shifted to higher temperatures at elevated pressures without any noticeable change in the depth of the $T_{1}$-minima (Fig. 76). Also the average correlation times $\langle\tau\rangle=\beta \tau_{\mathrm{m}}$ exhibit a pronounced nonArrhenius temperature dependence, which is usually observed in highly viscous liquids displaying non-exponential relaxation characteristics. Isochoric average correlation times $\langle\tau\rangle_{0}$ for glycerol- $d_{4}$ display a linear dependence on shear viscosity $\eta$ over a five orders of magnitude change in the latter quantity (Fig. 77) in accordance with simple hydrodynamic mode-coupling theories.
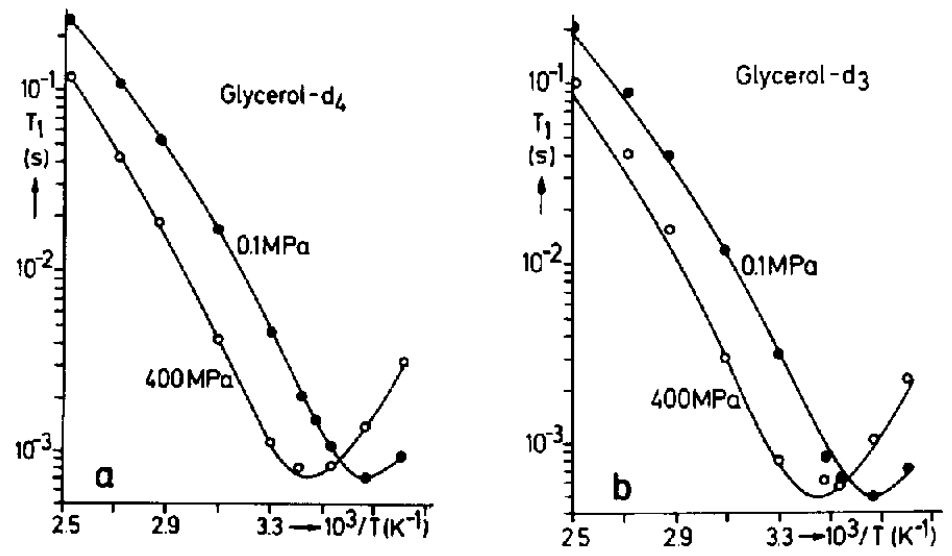

FIG. $76 .{ }^{2} \mathrm{H}-T_{1}$ isobars in selectively deuterated glycerol (a) glycerol- $\mathrm{d}_{4}$ (b) glycerol-d $\mathrm{d}_{3} \cdot{ }^{(435)}$ 


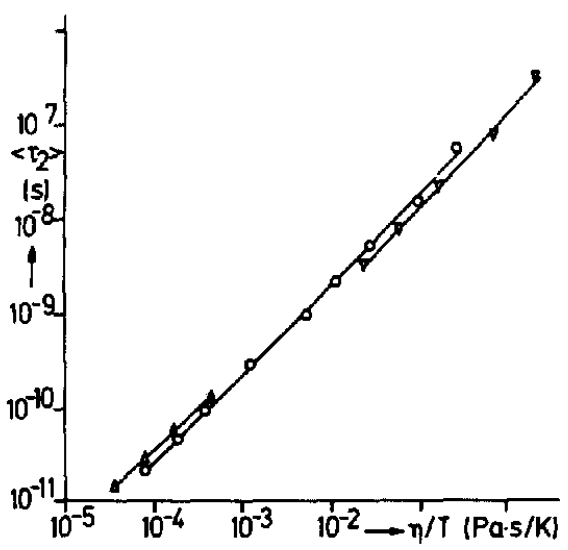

FIG. 77. Average orientational correlation time $\left\langle t_{2}\right\rangle$ versus $\eta / T$ in glycerol- $d_{4}$ at constant volume $(\Delta)$ $74 \times 10^{-6}\left(\mathrm{~m}^{3} / \mathrm{mol}\right),(0) 71 \times 10^{-6}\left(\mathrm{~m}^{3} / \mathrm{mol}\right),(\nabla) 68 \times 10^{-6}\left(\mathrm{~m}^{3} / \mathrm{mol}\right){ }^{(433)}$

The intercept parameter $\tau_{\mathbf{H}}(T)$ in the modified Debye-Stokes-Einstein equation

$$
\langle\tau\rangle=C_{\rho}(\eta / T)+\tau_{\mathrm{H}}(T)
$$

though not of fundamental physical significance ${ }^{(438)}$ has been shown in non-associated organic liquids ${ }^{(358)}$ to be of the order of the free rotor correlation time $\tau_{\mathrm{fr}}$ and should vary with temperature only slightly $\left(\tau_{\mathrm{fr}} \sim T^{-1 / 2}\right)$. In liquid glycerol, however, $\tau_{\mathrm{H}}(T)$ varies strongly with temperature and can be represented well by a VTF-equation. Since $\tau_{H}(T)$ does not contain density effects it was suggested that it reflects the influence of hydrogen bonding interactions upon reorientational motions. It is known from transport properties that hydrogen bonding interactions in liquid alcohols are not much affected by density variations but are strongly disturbed by increasing thermal energy. In contrast, the value of the slope parameter $C_{\rho}$ corresponds to those obtained in organic liquids, hence has been considered to reflect repulsive interactions. Considering the glycerol molecule as an ellipsoidal body the magnitude of $C_{\rho}$ can be estimated reasonably well indicating that the deuteron $T_{1}$ can indeed monitor single particle reorientations. This is further corroborated by a comparison of $\left\langle\tau_{2}\right\rangle$ with correlation times deduced from dielectric relaxation, viscoelastic and ultrasonic relaxation studies. ${ }^{(439,440)}$ A comparison of backbone and hydroxyl deuteron relaxation time curves (Fig. 76) shows that the $T_{1}$-minima in glycerol- $\mathrm{d}_{4}$ and glycerol- $\mathrm{d}_{3}$ occur at identical temperatures and pressures. This has been interpreted as an indication that the reorientation of the -CD and -OD groups in glycerol must be strongly correlated.

6.3.2. Ammonia. In contrast to liquid hydrogen sulphide, the hydrides of nitrogen, oxygen and fluorine form liquids with rather unique structures. The physical properties of these associated liquids are dominated by the ability of these hydrides to participate in strongly directional and saturable hydrogen bonding interactions. The rather unusual structure and dynamics of the three dimensional transient H-bond network in liquid water is well established. Compared to the 4-5 nearest neighbours in liquid water, the radial distribution function of liquid ammonia ${ }^{(441)}$ shows approximately 12 next neighbours, as generally found in simple liquids. Though liquid ammonia has been the subject of many investigations it is still in dispute whether its dynamic structure is dominated by $\mathbf{H}$-bonding interactions. ${ }^{(442-445)}$ Because NMR can provide valuable information about structure and dynamics in liquids many investigations of the temperature dependence of spin relaxation times have been performed. ${ }^{(442,443,446-448)}$ The only HP-NMR spin relaxation study of liquid ammonia has been reported by Hauer $e t$ al. ${ }^{(449)}$ Proton spin-lattice relaxation times in liquid $\mathrm{NH}_{3}$ have been measured at temperatures $T_{\mathrm{m}}(p) \leqslant T \leqslant 453 \mathrm{~K}$ and pressures $p \leqslant 250 \mathrm{MPa}$. Deuteron spin-lattice relaxation times have been studied also in liquid $\mathrm{ND}_{3}$ in the temperature range $T_{\mathrm{m}}(p) \leqslant T \leqslant 351 \mathrm{~K}$ at pressures 
$p \leqslant 250 \mathrm{MPa}$. Under these conditions the relaxation of the deuterons is dominated completely by electric quadrupole interactions (Fig. 78). In the low temperature range $(T<300 \mathrm{~K})$ the proton relaxation is determined largely by dipolar interactions but at high temperatures significant contributions from spin-rotation interactions have been found (Fig. 79). All relaxation rates obtained correspond to the fast motions regime, however. In order to deduce effective orientational correlation times from the ${ }^{2} \mathrm{H}-T_{1}$, the $\mathrm{QCC}$ in liquid $\mathrm{ND}_{3}$ has to be known. Powles et al. ${ }^{(447)}$ reported $\chi=245 \pm 25(\mathrm{kHz})$ from a comparison of proton and deuteron relaxation times assuming the equality $\tau_{2}^{\mathrm{DD}}\left({ }^{1} \mathrm{H}\right)=\tau \tau_{2}\left({ }^{2} \mathrm{H}\right)$. However, Atkins et al. ${ }^{(448)}$ later showed from a comparison of ${ }^{14} \mathrm{~N}-T_{1}$ in $\mathrm{NH}_{3}$ and $\mathrm{ND}_{3}$ that the orientational correlation times $\tau_{2}$ transform under isotopic substitution as the square root of the respective moments of inertia

$$
\frac{\tau_{2}\left(\mathrm{NH}_{3}\right)}{\tau_{2}\left(\mathrm{ND}_{3}\right)}=\left(\frac{l\left(\mathrm{NH}_{3}\right)}{l\left(\mathrm{ND}_{3}\right)}\right)^{1 / 2} .
$$

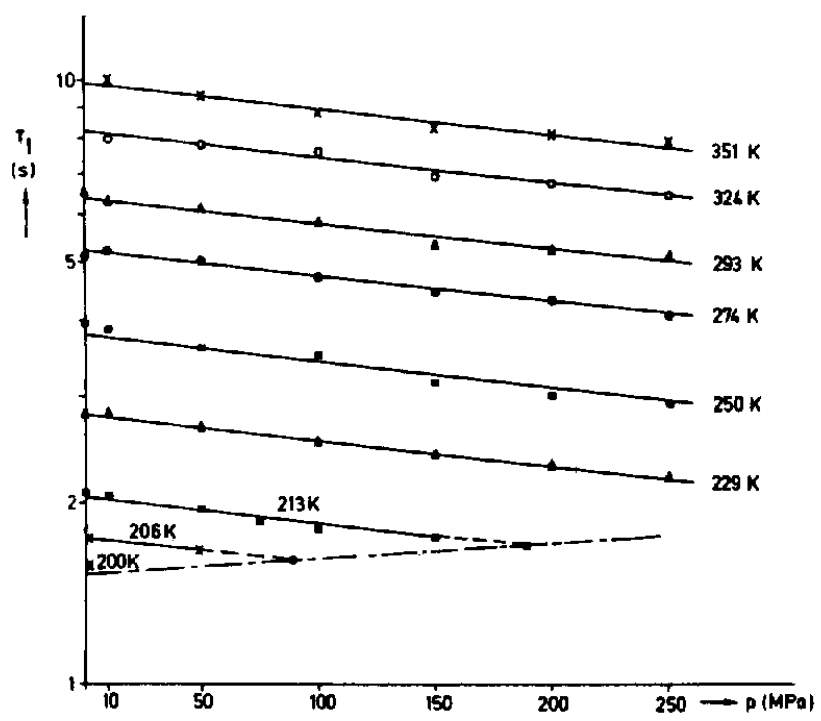

FIG. 78. Isothermal pressure dependence of the deuteron relaxation times $T_{1}$ in liquid deuteroammonia. ${ }^{(449)}$

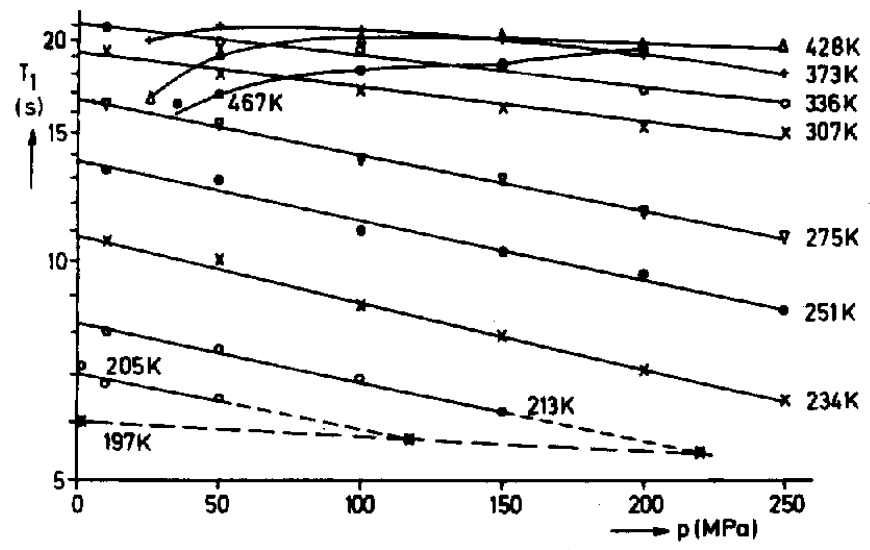

FIG. 79. Isothermal pressure dependence of the proton relaxation times $T_{1}$ in liquid ammonia. (449) 
Accounting for this isotope effect, Hauer et al..$^{(449)}$ proposed a QCC of the deuterons in liquid $\mathrm{ND}_{3}$ of $\chi=208 \pm 21(\mathrm{kHz})$. The latter value has been used to calculate effective orientational correlation times $\tau_{2}^{\mathrm{Q}}$. As the ammonia molecule is a symmetric top, $\tau_{2}^{\mathrm{Q}}$ contains contributions from reorientations around the $\mathrm{C}_{3}$-symmetry axis, $\tau_{\|}$, and a perpendicular axis, $\tau_{\perp}$. At ambient pressure both contributions and hence the motional anisotropy $\alpha=\tau_{\perp} / \tau_{||}$can be evaluated from deuteron and nitrogen-14 relaxation time data. Assuming that the principal axis systems of the moment of inertia tensor $I$ and the diffusion tensor $D$ coincide, it has been concluded that the motional anisotropy of rotational motions in liquid ammonia should be small, i.e. $\alpha \simeq 1$ (eqn (125)). Assuming the validity of this result in the whole $p, T$-range studied, effective orientational correlation times $\tau \frac{q}{q}\left(\mathrm{ND}_{3}\right)$ and $\tau_{2}^{\mathrm{DD}}\left(\mathrm{NH}_{3}\right)$ should be related by eqn (123). Thus intramolecular dipolar relaxation rates (eqn (45)) were obtained directly from measured deuteron relaxation rates. By applying the relation between orientational and angular momentum correlation times as given in the $J$-diffusion model ${ }^{(318)}$ appropriate for symmetric tops in the small angle diffusion limit $\left(\tau_{j}^{*} \ll 1\right)$ :

$$
\begin{gathered}
\tau_{2} \tau_{\mathrm{J}}=\frac{I_{\perp}}{6 k T} f_{2}(\theta, \alpha), \\
f_{2}(\theta, \alpha)=\frac{\left(3 \cos ^{2} \theta-1\right)^{2}}{4}+\frac{18 \sin ^{2} \theta \cos ^{2} \theta}{5+\alpha}+\frac{9 \sin ^{4} \theta}{4(1+2 \alpha)},
\end{gathered}
$$

( $\alpha=I_{\perp} / I_{||}, \theta$ is the angle of the $z$-axis of the efg-tensor in the inertia tensor principal frame) corresponding angular momentum correlation times $\tau_{\mathrm{J}}$ have been calculated. Irrespective of the motional model used to relate $\tau_{2}$ and $\tau_{j}{ }^{(450)}$ almost all $\tau_{2}^{*}$ have been found to fall in the region where the Hubbard relation holds. This renders small angle diffusion an adequate approximation of the rotational dynamics of the ammonia molecules over the $p, T$-range covered in this investigation.

The elements of the spin-rotation coupling tensor $C$ for the protons in $\mathrm{NH}_{3}$ are known in the principal inertial axis system from microwave data, ${ }^{(451)}$ hence spin-rotation relaxation rates $R_{1}$, SR can also be calculated (eqn (68)). Finally, intermolecular dipolar relaxation rates $R_{1 \text {, inter }}$ have been obtained by subtracting $R_{1}$, intra and $R_{1, \mathrm{SR}}$ from the total proton spin-lattice relaxation rate $R_{1}\left({ }^{1} \mathrm{H}\right)$ in liquid $\mathrm{NH}_{3}$. Thus a complete separation of all contributing relaxation rates could be obtained, as is shown in Fig. 80. As can be seen, the most effective relaxation mechanism of the proton spins is the modulation of their intermolecular dipolar interactions by translational motions of the molecules. Under ambient pressure conditions a linear correlation between $R_{1}$, inter and $(\rho \eta / T)$ is found, supporting the general validity of the hydrodynamic model (eqn (49)). Neither self-diffusion coefficient, $D$, nor shear viscosity, $\eta$, data exist at elevated pressures to test this relation further. This is unfortunate as the intermolecular dipolar relaxation contribution turns out to dominate even more at higher densities. Increasing temperature causes the intermolecular relaxation rate to decrease. At the highest temperatures measured the separation procedure led to an apparent increase of the intermolecular relaxation rate which was considered physically unacceptable, and points to the inadequacy of the assumptions underlying the separation procedure. Most notably, the small step diffusion model must fail and higher order approximations in any relation for $\tau_{2}\left(\tau_{\mathrm{J}}\right)$ have to be included. Calculated apparent activation enthalpies $\Delta H^{*}$ (eqn (109)) at constant pressure and constant density are rather similar $\left(\Delta H^{*} \simeq 6-7 \mathrm{~kJ} / \mathrm{mol}\right)$ for both translational and rotational motions, in contrast to simple liquids where the isobaric temperature coefficient $(\partial \ln \tau / \partial(1 / T))_{p}$ is twice as large as the isochoric equivalent. The values obtained are intermediate between those corresponding to liquid water $(\sim 14 \mathrm{~kJ} / \mathrm{mol})^{(452)}$ and to liquid hydrogen sulphide $(\sim 3 \mathrm{~kJ} / \mathrm{mol}) .^{(134)}$ Hence hydrogen bonding interactions hinder rotational and translational motions in liquid ammonia in much the same way. But the corresponding pressure coefficients $\Delta V^{*}$ (eqn (109)) are significantly smaller for rotational motions $\left(\Delta V_{\mathrm{rot}}^{*} \simeq 2-3 \mathrm{~cm}^{3} / \mathrm{mol}\right)$ than for translational motions $\left(\Delta V_{\text {trans }}^{*} \simeq 5 \mathrm{~cm}^{3} / \mathrm{mol}\right)$ indicating that the latter are slowed down to a greater extent with increasing density than are the former. This observation seems to be generally valid in molecular liquids.

6.3.3. Water. The hydride of oxygen is rather exceptional in that it is the only chemical species which exists in all three phases (gas, liquid, solid) in the natural environment. Hence $\mathrm{H}_{2} \mathrm{O}$ is of central 

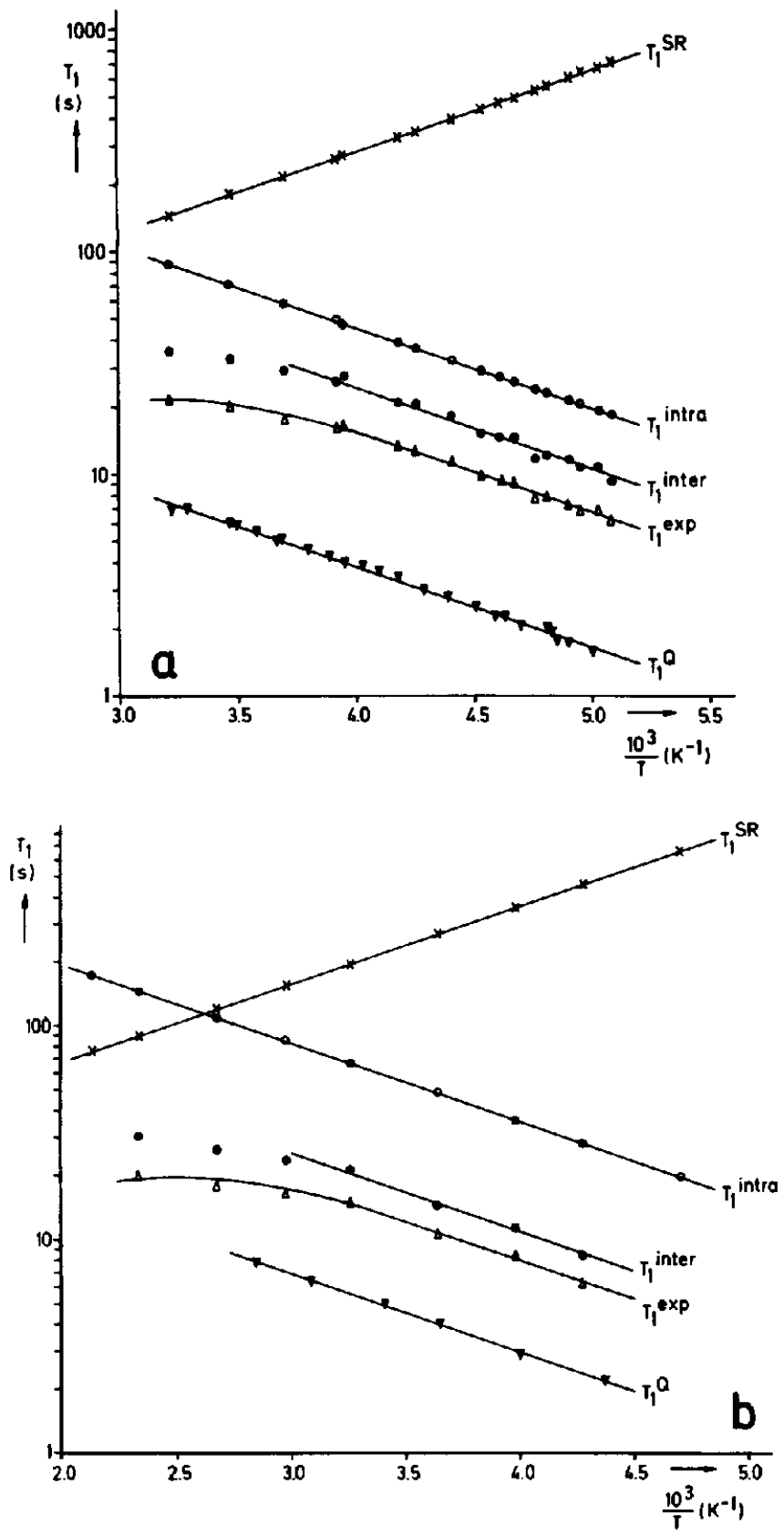

FIG. 80. Temperature dependence of the proton $\left(T_{1}^{\exp }\right)$ and deuteron $\left(T_{1}^{Q}\right)$ relaxation times in liquid ammonia at (a) saturation pressure and (b) $250 \mathrm{MPa}$. Relaxation times due to intra- and intermolecular dipolar as well as spin-rotation interactions are shown also. ${ }^{(449)}$

importance in many geochemical, geophysical and all biological processes. Besides its central role in many fields of science, liquid water is unique because of its three-dimensional random, transient H-bond network which is the basis of many of its peculiar physical properties. ${ }^{(453)}$ Not unexpectedly, water has been the subject of numerous NMR investigations since the early days of magnetic 
resonance. A review of HP-NMR studies of water has been given recently by Lang and Lüdemann. ${ }^{(454)}$ Benedek and Purcell ${ }^{(379)}$ were the first to report the pressure dependence of the proton spin-lattice relaxation time $T_{1}\left({ }^{1} \mathrm{H}\right)$ and self-diffusion coefficient $D_{\mathrm{s}}$ in the range $p \leqslant 1 \mathrm{GPa}$ at room temperature $(T=302 \mathrm{~K})$. Their results clearly demonstrate (Fig. 81) that the total proton relaxation rate does not follow the viscosity behaviour as suggested by Bloembergen et al. ${ }^{(81)}$ in applying simple hydrodynamic considerations to express rotational and translational correlation times via the Debye-Stokes-Einstein and Stokes-Einstein equations respectively.

They correctly concluded that intramolecular dipolar couplings provide the dominant relaxation mechanism, contrary to the hydrides of sulphur and nitrogen, and that compression of liquid water inhibits migrational freedom more drastically than rotational freedom. Though they realized the rather modest pressure dependence of $T_{1}$, their precision was not sufficient to detect the increase of $T_{1}$ upon initial compression at low temperatures which has been observed first by Hertz and Rädle. ${ }^{\text {(455) }}$ A shallow maximum in the self-diffusion coefficient $D$ at $273 \mathrm{~K}$ has also been reported by these authors. Considering the relative importance of intra- versus intermolecular dipolar contributions to the proton relaxation rate, Hertz and Rädle suggested that rotational diffusive motions of water molecules become faster with increasing density in cold water. Quadrupolar relaxation of the deuterons in heavy water is especially well suited to investigate the latter observation further in that it monitors single molecule reorientational mobility exclusively. Lee and Jonas ${ }^{(457)}$ performed the first HP-NMR experiments on liquid deuterium oxide $\left(\mathrm{D}_{2} \mathrm{O}\right)$.

In measuring the temperature and pressure dependence of the deuteron spin-lattice relaxation time and of the density and viscosity of $\mathrm{D}_{2} \mathrm{O}$ in the range $280 \mathrm{~K}<T<360 \mathrm{~K}$ and $p \leqslant 500 \mathrm{MPa}$ they were able to separate the effects of density and temperature on $T_{1}$ and $\eta$. These experiments were extended later by Jonas et al. ${ }^{(457)}$ and De Fries and Jonas ${ }^{(458)}$ to even higher pressures $(p \leqslant 900 \mathrm{MPa})$ and lower temperatures $T \geqslant 258 \mathrm{~K}$. Figure 82 collects the ${ }^{2} \mathrm{H}-T_{1}$ for $D_{2} \mathrm{O}$ measured by the group of Jonas in the thermodynamic stability range of liquid $\mathrm{D}_{2} \mathrm{O}$. The data have been discussed in terms of hydrodynamic concepts relating microscopic orientational correlation times $\tau_{2}$ to macroscopic shear viscosity coefficients. All relaxation rates collected were in the fast motions limit, hence Lee and Jonas considered the product

$$
\left(\eta T_{1}\left({ }^{2} \mathrm{H}\right) / T\right) \sim \chi^{2}\left(1+\eta^{2} / 3\right) \kappa
$$

which they found to be independent of temperature but to increase strongly with density.

From an earlier study of the pressure dependence of the proton $T_{1}$ in $\mathrm{H}_{2} \mathrm{O},{ }^{(459)}$ as well as from a consideration of the temperature independence of the quantities $(D \eta / T)$ and $\left(\tau_{\text {diel }} T / \eta\right),{ }^{(460,461)}$ Lee and Jonas concluded that rotational and translational motions must be strongly coupled $(\kappa \simeq 1)$ in liquid water irrespective of changes in the $\mathrm{H}$-bond network topology with changing temperature at constant density. The strong increase in the product $\left(\chi^{2}\right) \kappa$ with density has been tentatively considered a consequence of a dependence of $\chi(\rho)$ on density $\rho$ with $\kappa$ assumed not to be very sensitive to density

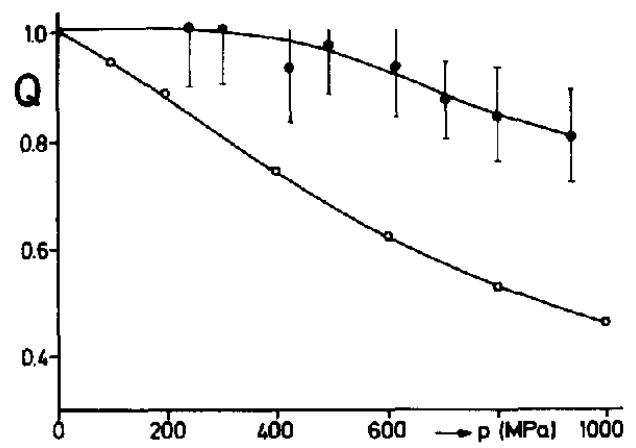

FIG. 81. Normalized proton relaxation times $Q=R(p) / R(0.1 \mathrm{MPa})(\mathrm{O})$ and self-diffusion coefficients $Q=D(p) / D(0.1 \mathrm{MPa})(\odot)$ as a function of pressure in liquid water. ${ }^{(379)}$ 


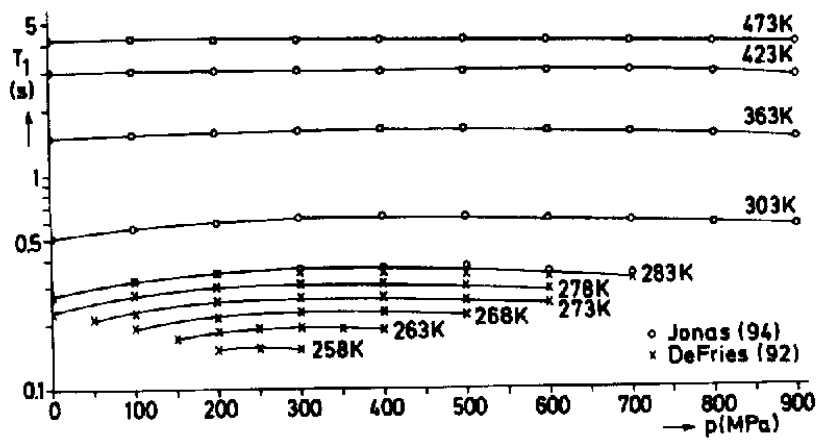

FiG. 82. Isothermal pressure dependence of the deuteron relaxation times $T_{1}$ of $\mathrm{D}_{2} \mathrm{O}$ in the thermodynamic stability range.

and temperature changes. Contrary to non-associated liquids discussed so far, $\mathrm{H}$-bonding interactions induce a gross redistribution of the electron density and a concomitant change of the $e f g$ at the deuterium nucleus resulting approximately in a $30 \%$ reduction of $\chi\left({ }^{2} \mathrm{H}\right)$ in going from the gas phase to the solid phase. ${ }^{(315)}$ A liquid state value of the $Q C C$ of $230 \mathrm{kHz}$ has been deduced by Powles $e$ al . $^{(447)}$ from a comparison of proton and deuteron $T_{1}$-data and the assumption that $\tau_{2}^{\mathrm{DD}}\left(\mathrm{H}_{2} \mathrm{O}\right)=\tau_{2}^{\mathrm{O}}\left(\mathrm{D}_{2} \mathrm{O}\right)$. A priori, the $Q C C$ might be expected to change with density and temperature in accordance with changes in the H-bond network topology, though one might expect that $\boldsymbol{\kappa}$ has to change also. With the assumption that there are no gross changes in the extent of $\mathrm{H}$-bonding interactions with density and temperature in liquid water, resulting in $x \simeq 1$, Lee and Jonas considered the possibility that at high hydrostatic pressures the $\mathrm{H}$-bond network undergoes some structural rearrangements that result in a shortened average distance $R_{\infty}$ in an H-bond. Scaling $R_{00} \sim \rho^{1 / 3}$ with the experimental density change $\left(1106 \mathrm{~kg} / \mathrm{m}^{3} \leqslant \rho \leqslant 1230 \mathrm{~kg} / \mathrm{m}^{3}\right)$ and using a relationship between the deuteron $Q C C$ and

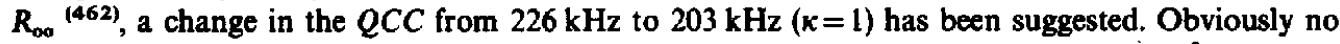
definite conclusions about any changes of $Q C C$ and/or $\kappa$ with density can be made from ${ }^{2} \mathrm{H}-T_{1}$ data alone. Therefore, Jonas et al. ${ }^{(457)}$ further investigated the proton and deuteron spin relaxation in compressed liquid water.

These studies were intended to provide an independent estimate of the effect of density and temperature on the rotation-translation coupling parameter $\kappa$. The proton $T_{1}$-data were in good agreement with results reported by Hindman et al. ${ }^{(\mathbf{4 6 3 )}}$ and Krynicki ${ }^{(464)}$ at ambient pressure, but differed from the high pressure results of Benedek and Purcell, ${ }^{(397)}$ and Kiselnik et al., ${ }^{(465)}$ who used non-degassed water, and also from the results of Hertz and Rädle, ${ }^{(45)}$ who reported a stronger pressure dependence of their proton $T_{1}$ at higher temperatures $(T \geqslant 300 \mathrm{~K})$. In order to separate interand intramolecular dipolar contributions Jonas et al. ${ }^{(457)}$ calculated the intermolecular dipolar relaxation rate with an expression introduced by Hubbard ${ }^{(104)}$ which includes both rotational and translational effects but assumes a uniform radial distribution function $\left(g(r)=1, r_{w}=1.38 \AA\right)$. Subtracting these calculated rates $R_{1 \text {, inter }}$ from the total proton relaxation rates yields $R_{1}$, intra and the coupling parameter $\boldsymbol{\kappa}$. The latter has now been found to decrease with increasing density

$$
\kappa\left(\mathrm{H}_{2} \mathrm{O}\right)=4.14-3.12 \rho\left(\mathrm{H}_{2} \mathrm{O}\right)
$$

and appears to be independent of temperature, as may be seen from the decreasing slope of $\tau_{2} \sim \kappa \eta$ in Fig. 83. With the coupling parameters $\kappa$ thus obtained, and neglecting any possible isotope effect, the deuteron $Q C C$ can be calculated. A constant average value of $\chi\left(1+\eta^{2 / 3}\right)^{1 / 2}=230 \mathrm{kHz}$ was obtained in the $p, T$-range $263 \leqslant T \leqslant 363 \mathrm{~K}$ and $p \leqslant 900 \mathrm{MPa}$ in good agreement with the value reported by Smith and Powles. ${ }^{(446)}$ The rather moderate variation of the deuterium $Q C C$ with density is in accord with computer simulations ${ }^{(446)}$ and neutron scattering ${ }^{(467)}$ results which report no variation of $R_{\infty 0}$ with pressure in liquid water. Figure 83 also displays a decrease of the reorientational correlation time $\tau_{2}$ 


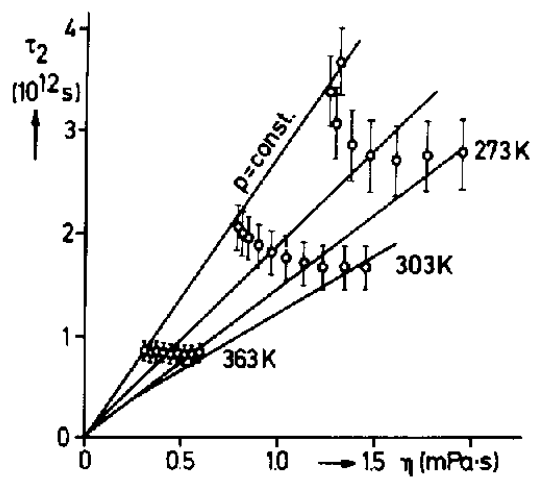

Fig. 83. Orientational correlation times $\tau_{2}$ versus $\eta$ in liquid water at three temperatures. ${ }^{(457)}$

with increasing viscosity. This behaviour is contrary to that of non-associated molecular liquids and corroborates the suggestion by Hertz and Rädle ${ }^{(455)}$ that isothermal compression of cold water facilitates molecular reorientational motions.

The latter unusual feature of the dynamics of liquid water has been further investigated by De Fries and Jonas ${ }^{\left(4 \xi^{8,468)}\right.}$ who studied the proton and deuteron spin-lattice relaxation times in $\mathrm{H}_{2} \mathrm{O}$ and $\mathrm{D}_{2} \mathrm{O}$ close to the melting pressure curves $T_{\mathrm{m}}(p)$ of the ice polymorphs, I, III and V. The data have been analyzed with respect to the applicability of hydrodynamic relations in much the same way as discussed by Jonas et al. ${ }^{(457)}$ Both proton and deuteron spin-lattice relaxation times, as well as self-diffusion coefficients, increase upon initial compression but decrease at high pressures. Shear viscosities show the inverse behaviour, of course. De Fries and Jonas noted that neither the temperature dependence of the extreme $\chi_{\max }$ nor their corresponding pressure values $p\left(\chi_{\max }\right)$ coincide. Accordingly, they could show that neither the Debye equation (eqn 22) nor the Stokes-Einstein equation (eqn 21) can reproduce the isothermal density dependence of the transport and relaxation coefficients, though isochoric correlations of $R_{1}\left({ }^{1} \mathrm{H}\right)$ and $R_{1}\left({ }^{2} \mathrm{H}\right)$ with $(\eta / T)$ were found to be linear. Thus the coupling of orientational and positional fluctuations seem to be independent of temperature but decrease with increasing density. At high densities next nearest neighbours came close to a central water molecule but nearest neighbours are almost unaffected. Hence $\mathrm{H}$-bonds are weakened by isothermal compression and repulsive interactions begin to dominate resulting in a dynamic structure of liquid water similar to non-associated molecular liquids.

De Fries and Jonas ${ }^{(458,468)}$ pointed out that pressure and temperature thus have similar effects on many dynamic properties of liquid water at low temperatures $(T<310 \mathrm{~K})$ and low pressures $(p<200 \mathrm{MPa})$ whereas at higher temperatures and pressures their effects are just the opposite. Obviously, cold water displays an unusual density and temperature dependence of its thermodynamic and transport properties and one might expect these characteristics to become even more pronounced in the undercooled, metastable state of liquid water. Rassmussen and McKenzie ${ }^{(469)}$ showed that water emulsified in cycloalkanes can be undercooled easily to $T_{\mathrm{H}} \simeq 235 \mathrm{~K}$ where homogeneous nucleation occurs at ambient pressure. Kanno et al. ${ }^{(\mathbf{4 7 0 )}}$ later followed the homogneous nucleation pressure curve $T_{\mathrm{H}}(p)$ to $180 \mathrm{~K}$ at a pressure of $p \simeq 200 \mathrm{MPa}$. Further compression then yields a positive slope of $T_{\mathrm{H}}(p)$. Though the emulsifying techniques render the preparation of metastable, undercooled water rather straightforward, only a few spectroscopic techniques can be applied. Out of these, NMR appears the most promising. Hence, Hindman et al. ${ }^{(463,471-473)}$ reported proton and deuteron $T_{1}$ measurements on undercooled water and ${ }^{17} \mathrm{O}-T_{1}$ measurements for oxygen-17 enriched water on $n$-heptane emulsions down to $T=242 \mathrm{~K}$. The first HP-NMR investigation of undercooled water was reported by Lang and Lüdemann. ${ }^{(452)}$ They measured the pressure and temperature dependence of proton spin-lattice relaxation times $T_{1}$ in undercooled water in the temperature range $186 \mathrm{~K} \leqslant T \leqslant 283 \mathrm{~K}$ and at pressures up to $p \leqslant 250 \mathrm{MPa}$ (Fig. 84). For the $200 \mathrm{MPa}$ isobar, a relaxation 


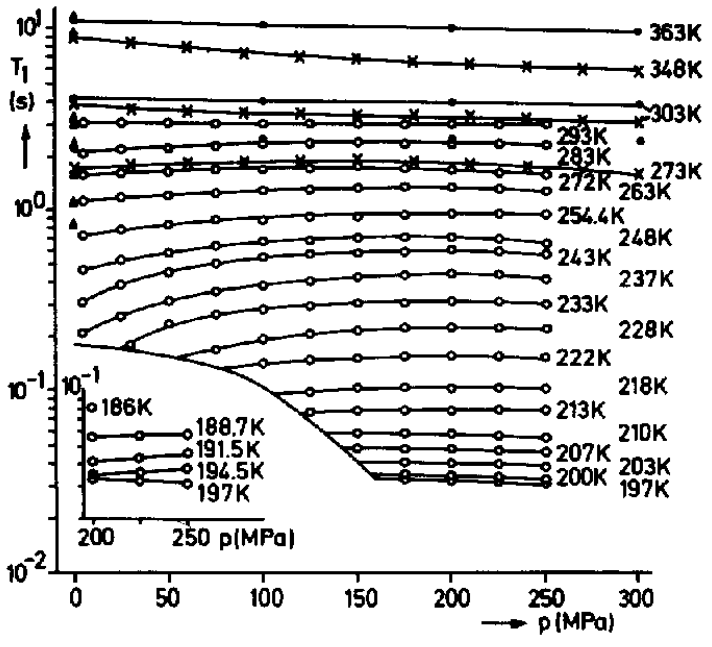

FrG. 84. Isothermal pressure dependence of the proton relaxation times $T_{1}$ in liquid water. ${ }^{(452)}$

rate maximum was observed for the first time in water indicating that the molecular dynamics are slowed down in a strongly non-Arrhenius fashion by three orders of magnitude from room temperature. Isothermal compression results in facilitated molecular motions. The latter effects is especially pronounced at temperatures $T \simeq 230-240 \mathrm{~K}$ and pressures $p \leqslant 200 \mathrm{MPa}$. As no further physical quantities were known in the $p, T$-range of metastable water investigated, a separation of intra- and intermolecular dipolar relaxation rates could not be achieved, of course.

In subsequent investigations Lang and Lüdemann ${ }^{(474-477)}$ further reported the pressure and temperature dependence of the deuterium spin-lattice relaxation times $R_{1}\left({ }^{2} \mathrm{H}\right)$ in undercooled heavy water ( $188 \mathrm{~K} \leqslant T \leqslant 282 \mathrm{~K}, p \leqslant 300 \mathrm{MPa})$ and of the ${ }^{17} \mathrm{O}-T_{1}$ in oxygen-17 enriched light and heavy water ( $238 \mathrm{~K} \leqslant T \leqslant 457 \mathrm{~K}, p \leqslant 250 \mathrm{MPa}$ ). Both nuclei monitor reorientational motions only, hence more definite conclusions about the density and temperature dependence of molecular motions as well as dynamic isotope effects could be given. The deuterium relaxation rates $R_{1}\left({ }^{2} \mathrm{H}\right)$ at high pressure $(p \geqslant 200 \mathrm{MPa})$ also display a maximum at temperatures close to the homogeneous nucleation curve $T_{\mathrm{H}}(p)$. For technical reasons, this maximum cannot be measured very precisely because of short $T_{1}$-values. Therefore, Lang et al..$^{(48,479)}$ in addition measured the proton and deuteron high pressure relaxation rate isobars in $\mathrm{D}_{2} \mathrm{O}, \mathrm{H}_{2} \mathrm{O}$ and $\mathrm{H}_{2}{ }^{17} \mathrm{O}$ at different Larmor frequencies.

In order to extract molecular as well as dynamic quantities from these relaxation coefficients, an appropriate motional model had to be chosen. According to the random network model, ${ }^{(480)}$ cold water may be considered a continuous random network of hydrogen-bonded molecules with their nearest neighbour arrangement corresponding on average to a tetrahedral coordination. Molecular motions may be divided into fast oscillatory motions and slow diffusional rearrangements. The short-time reorientational motions of the water molecules consists of fast librations about an equilibrium orientation in the quasistatic random network. On a longer time scale this equilibrium orientation will change due to slower rotational diffusive motions thereby leading to a permanent rearrangement of the network topology. Because of time scale separation, fast librations and slow diffusive tumbling motions are statistically independent. The fast oscillations average out part of the spin-lattice coupling, leading to an effective, librationally averaged, $e f g, q_{\mathrm{eff}}=q_{\mathrm{zz}}^{\mathrm{l}} \Gamma$ (eqn (58)) in the case of deuterium and oxygen-17 nuclei, and to an effective distance $r_{\text {eff }}^{-6}=\left(\Gamma / r_{\mathrm{HH}}^{3}\right)^{2}$ in the case of the protons, but make a negligible contribution to the spectral density at the Larmor frequency $g\left(\omega_{\mathbf{L}}\right)$.

Approximating the overall tumbling motions of the water molecules by an isotropic rotational diffusion process the corresponding relaxation rates of deuterium nuclei are given by eqn $(60)$. In the fast motions limit $\left(\omega_{\mathrm{L}} \tau_{2}<1\right)$ these expressions are also valid in case of oxygen-17 nuclei, the relaxation 
of which has not yet been followed into the slow motions regime. Spin-lattice and spin-spin relaxation rates of the deuterons in strongly undercooled heavy water under high pressure have been obtained at different Larmor frequencies (Fig. 85). They provide an estimate of the overall tumbling correlation time $\tau_{\text {rot }}$ independent of a knowledge of the $Q C C$ (Fig. 86). With the correlation times thus

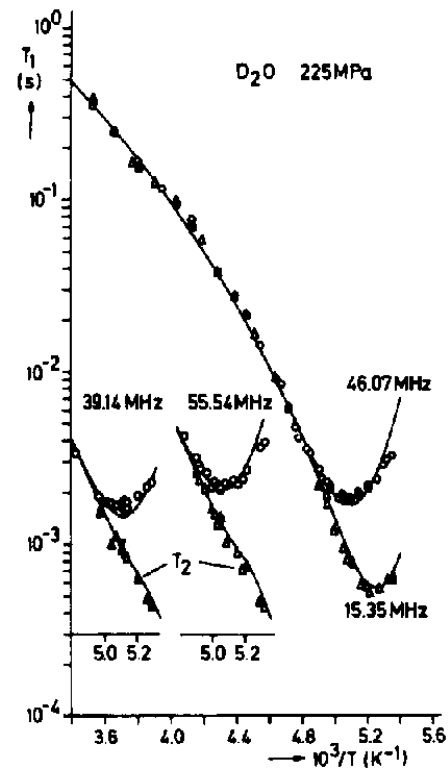

Fig. 85. High pressure isotherms of the deuteron spin-lattice $\left(T_{1}\right)$ and spin-spin $\left(T_{2}\right)$ relaxation times in undercooled heavy water at different Larmor frequencies. ${ }^{(478)}$

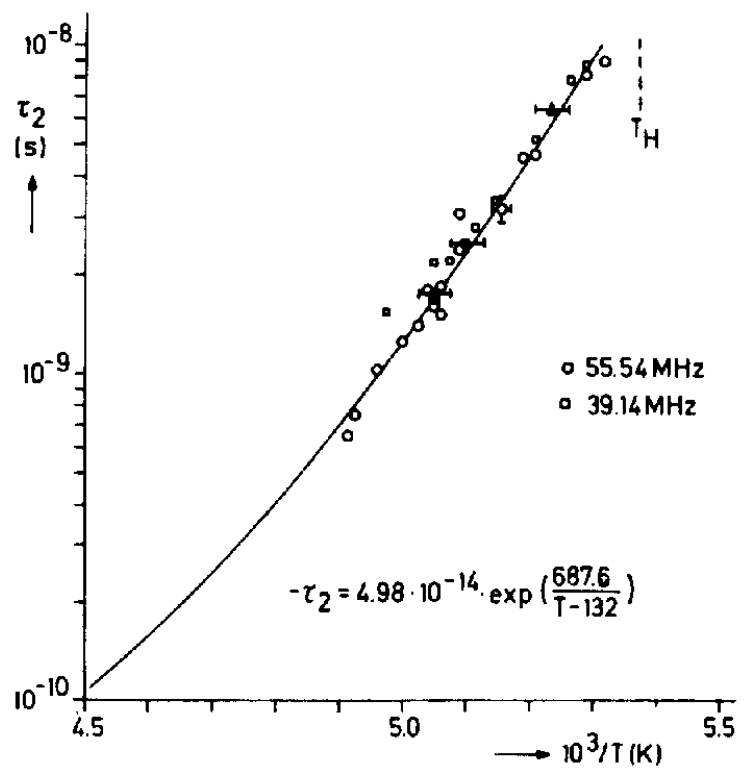

FIG. 86. Isobaric (225 MPa) temperature dependence of orientational correlation times of water as obtained from the experimental ratio $T_{1} / T_{2}$. Filled symbols give $\tau_{2}$ calculated from the minimum condition $\omega_{L} \tau_{2}=0.6158$. $T_{H}$ is the homogeneous nucleation temperature at $p=225 \mathrm{MPa}^{\left({ }^{(47)}\right.}$ 
obtained, the librationally averaged $Q C C$ can be derived in the temperature range $188 \mathrm{~K} \leqslant T \leqslant 205 \mathrm{~K}$ at $p=225 \mathrm{MPa}$ yielding an average value of $\chi\left({ }^{2} \mathrm{H}\right)=202 \pm 12 \mathrm{kHz}$. This value is lower than the coupling constants in ice II, $220-225 \mathrm{kHz},{ }^{(481)}$ the stable ice polymorph in the $p, T$-range mentioned above. It depends on the librational amplitude which in turn is influenced by the degree of distortion of the $\mathrm{H}$-bond according to well established correlations between $Q C C$ and geometrical $\mathrm{H}$-bond parameters. ${ }^{(478,482-484)}$ As the various geometric parameters and amplitudes of oscillation are determined by forces due to interactions with neighbouring molecules, Lang et al. ${ }^{(48)}$ argued that the correlation of $Q C C$ with $\mathrm{H}$-bond parameters will be the same whether the changes are caused by varying the temperature and the pressure or by changing the phase.

Hence, these correlations can be used to perform an order of magnitude estimate of the variation of $Q C C$ that could possibly be induced within the $p, T$-range covered by the experiments. The resulting variations turn out to be negligibly small. Furthermore, as the librational averaging mechanism predicts an opposite temperature dependence to that expected from correlations with geometric H-bond parameters, it seems to suggest that the $Q C C$ can be considered a $T$ - and $p$-independent coupling strength to a good approximation. The latter has been also tacitly assumed in cases of dipolar couplings. ${ }^{(47,479)}$ The isobaric temperature dependence of the overall tumbling correlation time $\tau_{2}(T, p)$, characteristic of single particle reorientations in a strongly disturbed $\mathbf{H}$-bond network, is well represented by the known VTF-equation:

$$
\tau_{2}=\left(2 \pi /\left\langle\omega_{\mathrm{lib}}\right\rangle\right) \exp \left(B(p) / T-T_{\mathrm{o}}(p)\right)
$$

with $T_{\mathrm{v}}$ representing a kinetic localization temperature below which the system becomes arrested in phase space in one of the minima of the potential hypersurface corresponding to a random structural arrangement of the molecules.

A best fit value of $T_{0}(225 \mathrm{MPa})=132 \mathrm{~K}$ in heavy water has been found in good agreement with estimates available in the literature. The pre-exponential factor of the VTF-equation can be associated with a typical librational period characterizing the fast orientational fluctuations of the water molecules in their potential wells of the quasistatic random network. With these ingredients, the shape of the isobaric relaxation rate curve $R_{1}\left({ }^{2} \mathrm{H}\right)$, as well as its variation with the Larmor frequency, can be reproduced very well, indicating that an isotropic diffusion model is sufficient to describe the single particle dynamics within the random, transient $\mathrm{H}$-bond network on a nanosecond time scale appropriate to the NMR experiment $\left(t \sim 2 \pi / \omega_{\mathrm{L}}\right)$.

Under ambient pressure conditions undercooled water is known to approach an apparent singularity $T_{\mathrm{s}}$ close to but below the homogeneous nucleation temperature $T_{\mathrm{H}}{ }^{(485)} T_{\mathrm{S}}(p)$ has been interpreted as a spinodal line by Speedy ${ }^{(486)}$ or as a percolation threshold by Stanley and Teixeira. ${ }^{(62)}$ Speedy and Angell ${ }^{(487)}$ suggested a fractional power law dependence for the isobaric temperature dependence of static and dynamic response functions in undercooled water. Lang and Lüdemann ${ }^{(475,476,479)}$ demonstrated convincingly that the fractional power law description of the correlation times $\tau_{2}$

$$
\tau_{2}=\tau_{\mathrm{s}} \varepsilon^{-\gamma}, \quad \varepsilon=\left(T-T_{\mathrm{s}}\right) / T_{\mathrm{s}}
$$

is superior to a VTF-equation in a largely undistorted random H-bond network, i.e. undercooled water at low pressures $p \leqslant 150 \mathrm{MPa}$, but that the reverse is true at high pressures (Fig. 87). The singular temperatures $T_{s}(p)$ have been found to follow approximately the homogeneous nucleation pressure curve $T_{\mathrm{H}}(p)$, as is shown in a supplemented phase diagram in Fig. 88.

The dynamic isotope effect observed for transport and relaxation coefficients in light and heavy water can be obtained directly from a comparison of ${ }^{17} \mathrm{O}-T_{1}$ in $\mathrm{H}_{2}{ }^{17} \mathrm{O}$ and $\mathrm{D}_{2}{ }^{17} \mathrm{O}$ in the case of rotational motions, and from a comparison of corresponding self-diffusion coefficients in the case of translational motions. It was observed that these dynamic quantities become numerically identical if compared at equal reduced temperatures $\varepsilon$, with the difference $\Delta T_{\mathrm{s}} \simeq 7 \mathrm{~K}$ corresponding to the difference in zero point energy of light and heavy water. This finding is a natural consequence of the dominance of hydrogen-bonding interactions on the dynamic structure of liquid water and suggests that orientational fluctuations are at the heart of all anomalous properties of cold water. These aspects have been neatly tied together recently by Robinson et al. ${ }^{(\mathbf{4 8 8}-491)}$ 


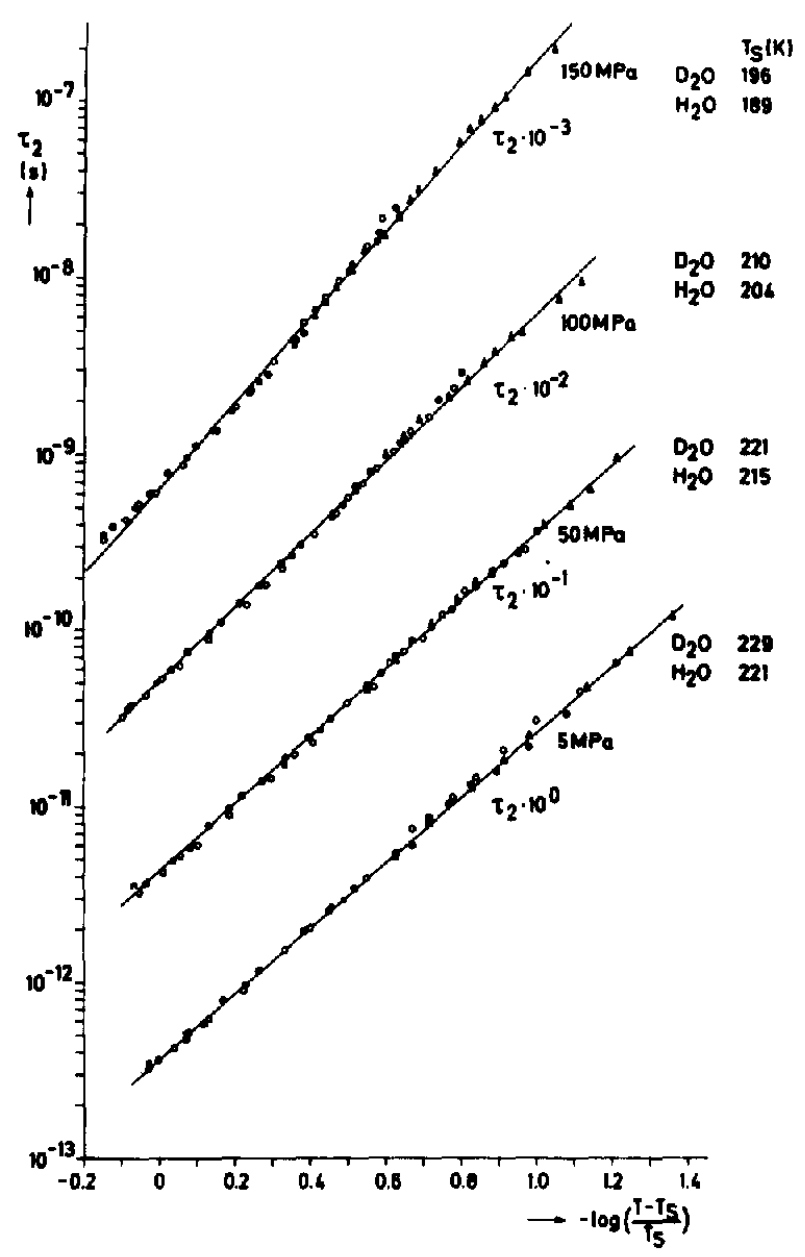

FIG. 87. Power law representation of isobars of orientational correlation times $\tau_{2}$ in $\mathrm{H}_{2} \mathrm{O}$ and $\mathrm{D}_{2} \mathrm{O}$ as obtained from ${ }^{17} \mathrm{O}-T_{1}$ in $\mathrm{H}_{2}{ }^{17} \mathrm{O}$ and $\mathrm{D}_{2}{ }^{17} \mathrm{O}$ and from ${ }^{2} \mathrm{H}-T_{1}$ in $\mathrm{D}_{2} \mathrm{O} .{ }^{(476)}$

A direct comparison of the isothermal pressure dependence of rotational $\left(D_{\mathrm{r}} \sim 1 / \tau_{\mathrm{rot}}\right)$ and translational $\left(D_{\mathrm{s}}\right)$ transport coefficients reveal another aspect of the dynamic structure of the random $\mathrm{H}$-bond network. At high temperatures thermal excitations strongly disrupt the network and compression leads to a reduced molecular mobility. As is also found in other molecular liquids of similar size and dipole moment, increasing pressure reduces the translational diffusion significantly more than does the rotational diffusion (Fig. 89). In undercooled water, thermal excitations are reduced and the $\mathrm{H}$-bond network is well developed. Initial compression is found to facilitate molecular motions and the effect is strikingly more pronounced for rotational than for translational motions. The explanation given was that compression brings next nearest neighbours closer to a central molecule with nearest neighbour correlations being largely unchanged. The potential energy surface is then less sharply partitioned and there is a greater variety of orientational states, separated by smaller energy barriers, so that rotational diffusion is enhanced. Enhancement of local molecular reorientations upon initial compression must also facilitate translational motions. But an increasing number of next neighbours must form obstacles for positional rearrangements. Thus self-diffusion starts to decrease even at pressures where rotation is still enhanced by further compression. Furthermore, these pressure effects 


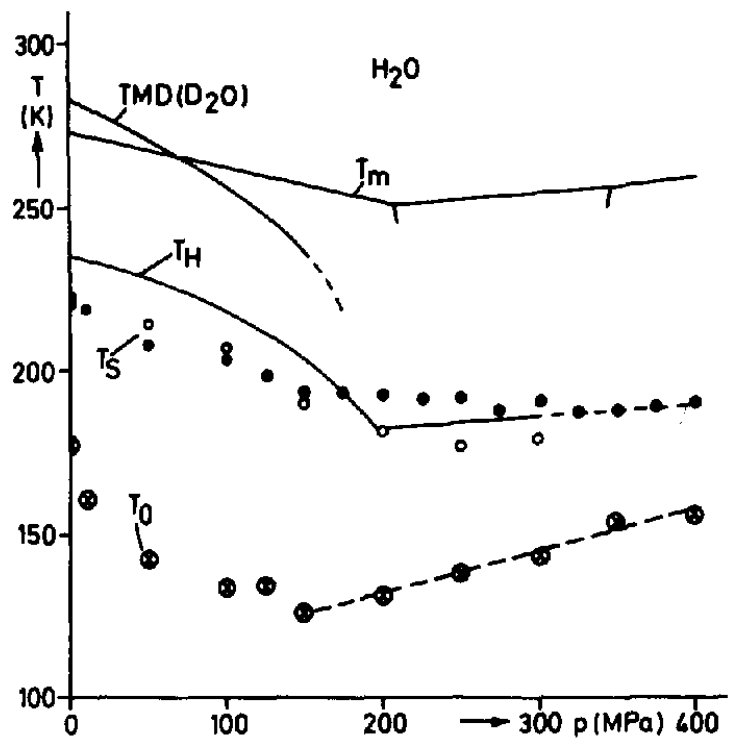

FIG. 88. Supplemented phase diagram of water showing the singular temperatures $T$, and the ideal glass transition temperatures $T_{0},{ }^{(18)}$

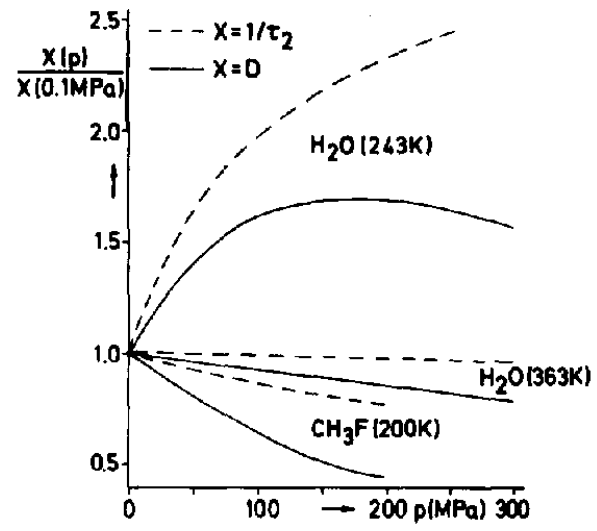

FIG. 89. Pressure dependence of reduced rotational mobilities $\left(1 / \tau_{2}\right)$ and self-diffusion coefficients $D$ of water in its stable and metastable phase. ${ }^{(18)}$

upon orientational and positional molecular displacements are the most direct indication of the failure of simple hydrodynamic mode-coupling relations (eqns $(21,22)$ ) to account for density effects on transport coefficients in cold water.

Proton relaxation rate measurements in $\mathrm{H}$-bonded liquids are especially difficult to interpret as a separation of inter- and intramolecular dipolar contributions to the relaxation rate cannot be achieved by the usual isotopic dilution technique because of rapid proton exchange. Because of the large dynamic isotope effect deuterium relaxation rates must not be used to estimate the intramolecular contribution which also monitors reorientational motions only. A solution to this problem was proposed by Lang and Lüdemann ${ }^{(474,476,477)}$ and fully developed by Lankhorst et al. ${ }^{(492)}$ Lang et al. $^{(479)}$ applied this approach in a HP-NMR study of undercooled light water to interpret proton 
relaxation rates in $\mathrm{H}_{2} \mathrm{O}$ and $\mathrm{H}_{2}{ }^{17} \mathrm{O}$ investigated as a function of pressure ( $p \leqslant 250 \mathrm{MPa}$ ), temperature $(180 \mathrm{~K} \leqslant T \leqslant 300 \mathrm{~K})$ and Larmor frequency $\left(100 \mathrm{MHz} \leqslant \omega_{\mathrm{L}} \leqslant 500 \mathrm{MHz}\right)$. Oxygen-17 enrichment in light water provides an additional relaxation pathway to the proton spin system. This ${ }^{1} \mathrm{H}^{-17} \mathrm{O}$ dipolar interaction is completely intramolecular in origin. Moreover it monitors the reorientation of the same interaction vector within the water molecule as is the case with the deuterium relaxation. This allows the dynamic isotope effect to be followed to the lowest temperatures obtainable. By studying the difference of the proton relaxation rates in $\mathrm{H}_{2}{ }^{16} \mathrm{O}$ and $\mathrm{H}_{2}{ }^{17} \mathrm{O}$, the additional ${ }^{1} \mathrm{H}-{ }^{17} \mathrm{O}$ intramolecular dipolar contribution may be obtained according to:

$$
\begin{gathered}
R_{1}\left(\mathrm{H}_{2}{ }^{16} \mathrm{O}\right)=R_{1, \text { OR }}\left({ }^{1} \mathrm{H}-{ }^{1} \mathrm{H}\right)+R_{1, \text { POS }}, \\
R_{1}\left(\mathrm{H}_{2}{ }^{17} \mathrm{O}\right)=R_{1, \text { OR }}\left({ }^{1} \mathrm{H}-{ }^{1} \mathrm{H}\right)+X_{\mathrm{O}-17} R_{1, \mathrm{OR}}\left({ }^{1} \mathrm{H}^{17} \mathrm{O}\right)+R_{1, \text { POs }},
\end{gathered}
$$

with $X_{\mathrm{O}-17}$ the mole fraction of oxygen-17 enriched water. At pressures $p \leqslant 150 \mathrm{MPa}$ all relaxation rates correspond to the fast motions limit, hence average orientational correlation times $\tau_{\text {or }}$ can be calculated with eqns $(180,131)$ and eqn (45). A static $\mathrm{OH}$-bond length $R_{\mathrm{OH}}=0.098 \mathrm{~nm}^{(493)}$ has been used in these calculations, as the effect of the librations is negligible at high temperatures due to strong damping. The resultant correlation times $\tau_{O R}$ were in quantitative agreement with correlation times deduced from spin-lattice relaxation rates $R_{1}, \mathrm{Q}\left({ }^{17} \mathrm{O}\right)$ in $\mathrm{H}_{2}{ }^{17} \mathrm{O}$ as may be seen in Fig. 90 . The isobaric

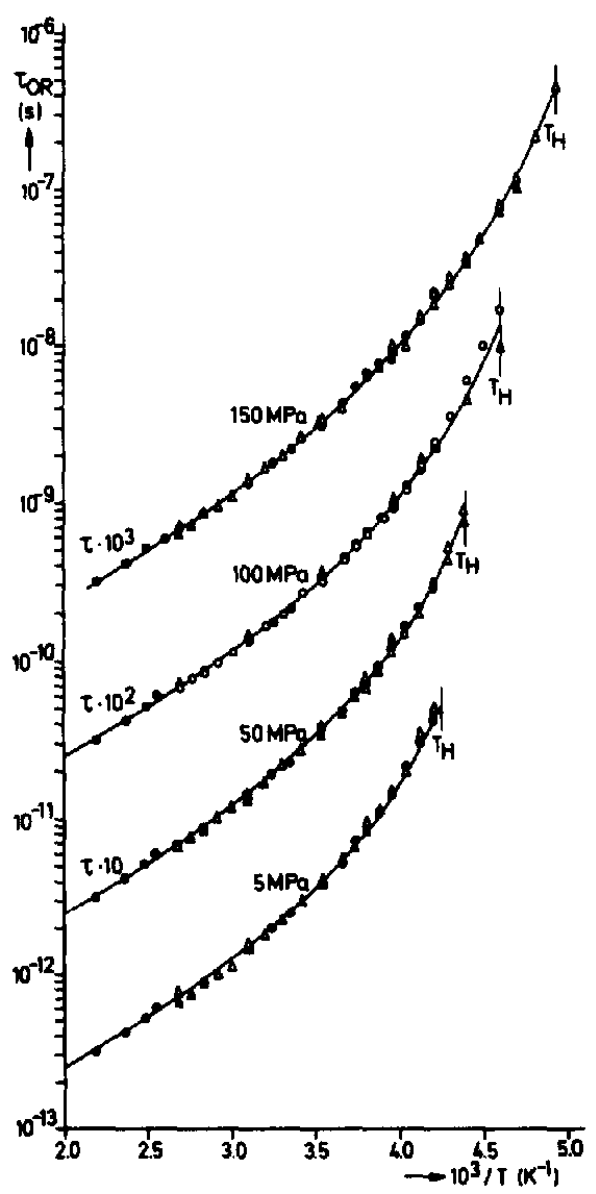

FIG. 90. Isobaric temperature dependence of orientational correlation times $\tau_{2}$ in under-cooled water as obtained from $(O)^{17} \mathrm{O}$ - and $(O, \Delta){ }^{1} \mathrm{H}-T_{1}, T_{\mathrm{H}}$ represents the corresponding homogeneous nucleation temperature. ${ }^{(479)}$ 
temperature dependence of the correlation times could be reproduced very well using the fractional power law (eqn 129) and the parameter $\tau_{\mathrm{s}}$ and $T_{\mathrm{s}}$ used to fit the ${ }^{17} \mathrm{O}-T_{1}$ data. ${ }^{(474,476,479)}$

High hydrostatic pressure disturbs the random, transient $\mathrm{H}$-bond network and suppresses the formation of correlated low density patches of tetrahedrally coordinated water molecules, Low thermal excitations cause slow molecular motions and allow the slow motion regime $\left(\omega_{\mathrm{L}} \tau>1\right)$ to be reached with modern NMR spectrometers. Under these conditions, the relaxation rate curves $R_{1}\left(\mathrm{H}_{2}{ }^{16} \mathrm{O}\right)$ and $R_{1}\left(\mathrm{H}_{2}{ }^{17} \mathrm{O}\right)$ are sensitive to details of the spin-lattice coupling and the molecular motions which mediate its time-dependence. Relaxation rate curves were sampled at five different Larmor frequencies and are shown in Fig. 91. Within an isotropic diffusion model both sets of data could be described consistently using eqns $(130,131,45$ and 48$)$, as discussed at length by Lang et al. ${ }^{(49)}$

From the maxima of the relaxation rate curves, librationally averaged distances $\left\langle R_{\mathrm{OH}}\right\rangle=0.106 \mathrm{~nm}$ and $\left\langle\boldsymbol{R}_{\mathbf{H H}}\right\rangle=0.159 \mathrm{~nm}$ were obtained. Approximating the motional averaging factor $\Gamma$ (eqn 59 ) by:

$$
\Gamma^{2}\left(\mathrm{H}_{2} \mathrm{O}\right)=1-(3 / 2)\left(\mathrm{h} / I \omega_{1 \mathrm{ih}}\right)=0.82, \quad T \leqslant 200 \mathrm{~K}
$$

a static bond length $R_{\mathrm{OH}}=0.098 \mathrm{~nm}$ was calculated, which is in good agreement with results obtained from neutron scattering data at room temperature. ${ }^{(493)}$ The isobaric temperature dependence of the correlation times $\tau_{\mathrm{OR}}$ in eqn (130) and eqn (131) and the self-diffusion coefficients $D_{\mathrm{s}}$ have been described by the VTF-equation (eqn (25)) with the glass transition temperature $T_{\mathrm{o}}$ rescaled by $\Delta T_{0}=T_{0}\left(\mathrm{D}_{2} \mathrm{O}\right)-T_{0}\left(\mathrm{H}_{2} \mathrm{O}\right)=7 \mathrm{~K}$ to account for the dynamic isotope effect. To calculate the intramolecular and intermolecular proton relaxation rates in light water it was assumed that $\tau_{\mathrm{OR}}(\mathrm{OH})=\tau_{\mathrm{OR}}(\mathrm{HH})$ in accord with an isotropic diffusion model. The spin density $N_{\mathrm{H}}$ (eqn (49)) was obtained from the mass density $\rho$ of water under high pressure, ${ }^{(458)}$ neglecting any weak temperature dependence. Also, the average distance of closest approach $d_{\mathrm{HH}}$ of two protons on different water molecules was taken as twice the van der Waals radius $\left(R_{\mathrm{W}}=0.138 \mathrm{~nm}\right)$, neglecting any nearest neighbour correlations. The resulting translational and rotational contributions to the proton relaxation rate in undercooled light water under a pressure of $p=200 \mathrm{MPa}$ are shown in Fig. 92.
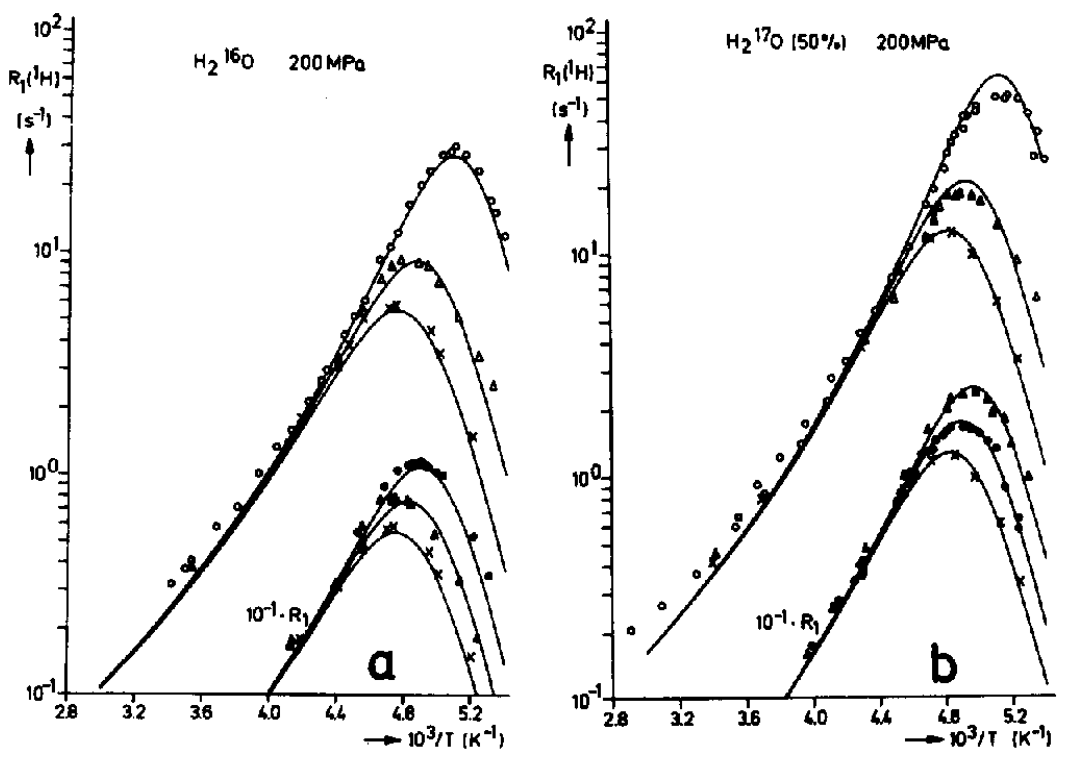

FIG. 91. Proton relaxation rate isobars (200 MPa) in undercooled (a) light water and (b) oxygen-17 enriched light water at five different Larmor frequencies. ${ }^{(479)}$ (a) $(0100 \mathrm{MHz} \& 300 \mathrm{MHz} ; \times 500 \mathrm{MHz}$; $255 \mathrm{MHz}$; $\triangle 362 \mathrm{MHz}$ (b) (๑ $100 \mathrm{MHz} ; 300 \mathrm{MHz} ; 500 \mathrm{MHz} ; \triangle 255 \mathrm{MHz} ; 0362 \mathrm{MHz})$. 


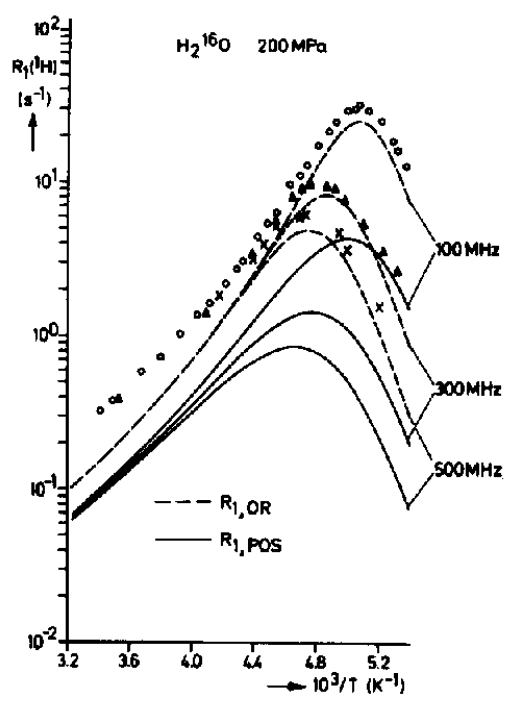

FIG. 92. Intra $\left(R_{1, O R}\right)$ and intermolecular $\left(R_{1, \text { POS }}\right)$ contributions to the total dipolar proton relaxation rate in undercooled water under high pressure $(200 \mathrm{MPa})$ at three different Larmor frequencies. ${ }^{(479)}$

It is seen that intramolecular dipolar couplings modulated by orientational fluctuations dominate the proton relaxation in the slow motion regime where relaxation due to positional fluctuations becomes almost negligible. At ambient temperatures both contributions are about equal. A very good representation of the shape of the relaxation rate curves at the different Larmor frequencies was achieved, corroborating the isotropy of the diffusional rearrangements of the water molecules in the $H$-bond network. Further, the necessity to include quasi-lattice vibrations into a consistent analysis of the relaxation rate curves $R_{1}\left(T, p, \omega_{L}\right)$ in the slow motion regime is demonstrated. The analysis remains approximate in that the strong damping of the librations at high temperatures was not taken into account, and this leads to small deviations in the calculated relaxation rate curves from the experimental data. With the static distances $R_{\mathrm{OH}}=0.98 \mathrm{~nm}$ and $R_{\mathrm{HH}}=0.156 \mathrm{~nm}$ an almost perfect agreement with the high temperature data is obtained, but the maximum relaxation rates are grossly overestimated. These results suggest that the effective distances $\left\langle R_{\mathrm{OH}}\right\rangle,\left\langle R_{\mathrm{HH}}\right\rangle$, and by analogy the effective quadrupole coupling constants $\langle\chi\rangle$, should exhibit a temperature dependence at constant pressure. These questions, concerning a possible pressure and temperature dependence of dipolar and quadrupolar coupling strengths in liquid water due to $\mathrm{H}$-bonding interactions, are still unresolved and await clarification by even more precise experiments.

Proton relaxation times in liquid water have been obtained as a function of pressure in the supercritical fluid phase at temperatures up to $973 \mathrm{~K}$ by Jonas et al., ${ }^{(494)}$ and Lamb and Jonas. ${ }^{(495)}$ Their ${ }^{1} \mathrm{H}-T_{1}$ isotherms are given as a function of density $\rho$ in Fig. 93. For $T \leqslant 500 \mathrm{~K}$, dipolar relaxation dominates the results, but these couplings become less important at higher temperatures/lower densities where the molecules can reorient rather freely. The relaxation is then dominated by the spin-rotation relaxation $R_{1}^{\mathbf{S R}}$ (eqn 68 ) which is enhanced by rising temperature and decreasing density.

The latter relaxation mechanism dominates the proton relaxation at temperatures $T>600 \mathrm{~K}$ and densities below twice the critical density $\left(\rho<2 \rho_{\mathrm{c}}\right)$. From these high temperature data the angular momentum correlation time $\tau_{\mathrm{J}}$ and its hard spheres analog, the Enskog correlation time $\tau_{\mathbf{E}}$ (eqn 73), were derived within a spherical top approximation, neglecting all anisotropic contributions to the spin rotation interaction tensor. The relaxation times $T_{1}^{\mathbf{S R}}$ show a linear dependence on density up to rather high densities $\left(\rho \leqslant 1.5 \rho_{\mathrm{c}}\right)$ and suggested an approach employed to interpret relaxation data of dilute gases. ${ }^{(339,496)}$ In the latter systems with exponentially decaying angular momentum correlation 


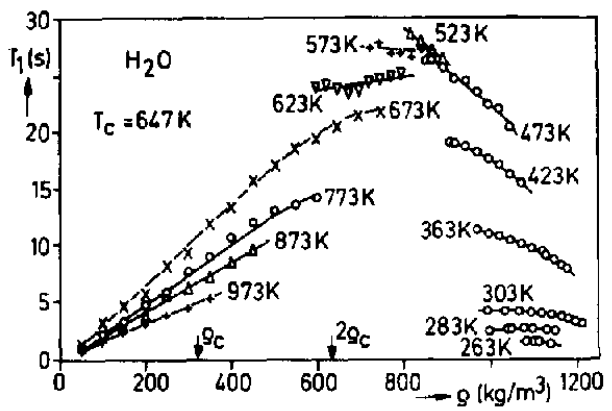

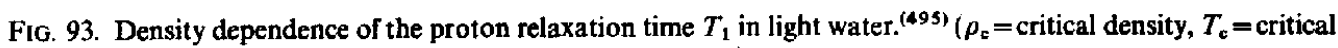
temperature).

functions and isotropic hard sphere interactions it was found that the relation

$$
T_{i}^{\mathrm{SR}} \sim \rho T^{-\gamma}, \quad \gamma \simeq 3 / 2
$$

holds to a good approximation. The exponent $y$ depends, however, on the nature of the intermolecular potential ${ }^{(496)}$ and has been found to be $\gamma=2$ in the case of compressed supercritical water in accord with a noticeable anisotropy in the intermolecular interactions of water even at supercritical temperatures (Fig. 94a). Still the linear dependence of $R_{1}^{\text {SR }}$ on the Enskog correlation time (Fig. 94b) suggested an evaluation of the data in terms of modified hard sphere theories, though drastic simplifications had to be made. ${ }^{(495)}$ In calculating the Enskog correlation times (eqn 73), hard sphere diameters have been calculated from gas solubility data. ${ }^{(416)} \mathrm{A}$ plot of the ratio $\tau_{\mathrm{Z}} / \tau_{\mathrm{J}}$ reflects the efficiency of angular momentum transfer as a function of density.

The strong density dependence of $\tau_{\mathrm{E}} / \tau_{\mathrm{J}}$ demonstrated the breakdown of the rough hard spheres approximation for supercritical water. As the latter are valid only for densities $\rho>2 \rho_{\mathrm{c}}$ attractive forces have to be taken into account yielding

$$
1 / \tau_{\mathrm{j}}=b(T, \rho)\left(1 / \tau_{\mathrm{E}}\right),
$$

where $b(T, \rho)=a(T) \exp \left(V_{\mathrm{L}}(\sigma)\right) . V_{\mathrm{L}}(r)$ is the renormalized intermolecular potential ${ }^{(497)}$ which is density
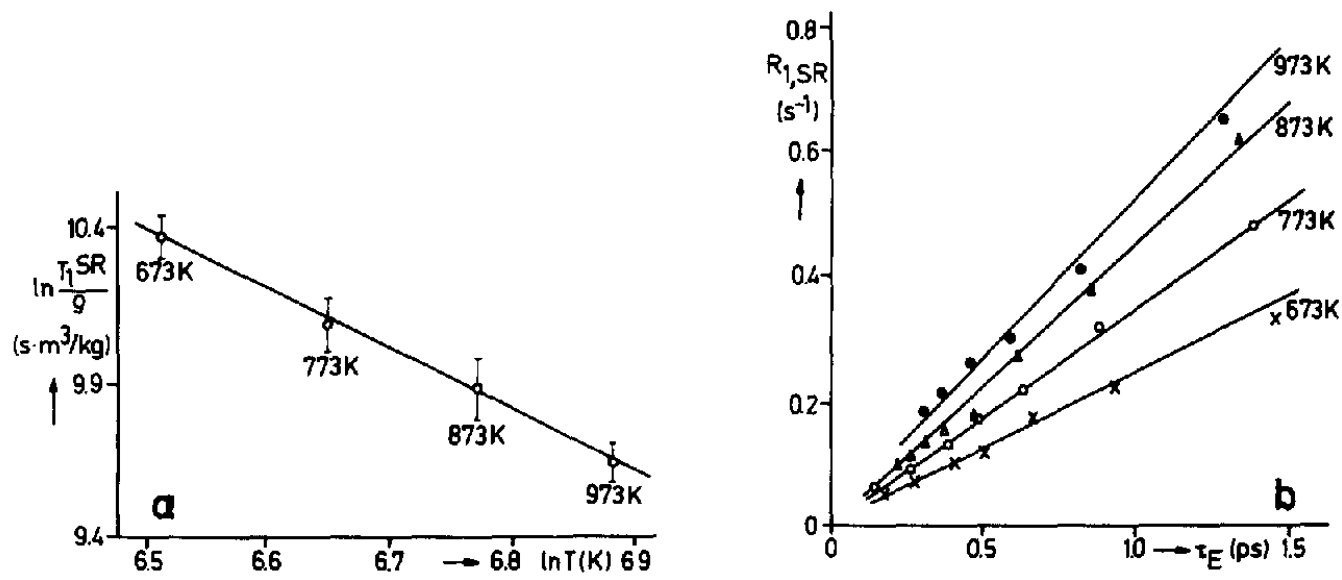

FIG. 94. (a) Plot of $\ln \left(T_{1, \text { sa }} / \rho\right)$ versus $\ln T$ for supercritical water. ${ }^{\text {(495) }}$ (b) Isotherms of the proton relaxation rate $R_{1,32}$ of supercritical water as a function of the Enskog correlation time $\tau_{E}$. ${ }^{(49)}$ 


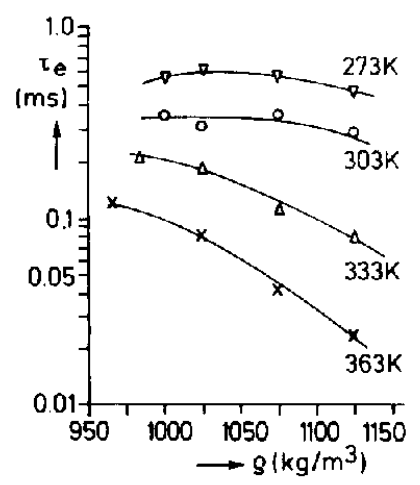

FIG. 95. Density dependence of the proton exchange lifetime $\tau_{e}$ in liquid water. ${ }^{\left({ }^{(508)}\right.}$

and temperature dependent. $V_{\mathrm{L}}(\sigma)=\varepsilon / k T$ for an infinitely dilute gas, and $V_{\mathrm{L}}(\sigma) \simeq 0$ for a dense fluid. Though large discrepancies between experimental data and theoretical calculations remain, because of the drastic simplifications made, the essential physical effects causing these deviations from a hard spheres behaviour are correctly described. Hence the authors conclude that future attempts to derive a rigorous theoretical treatment of spin-rotation relaxation data in supercritical water are well worth the effort. This effort, however, has not yet been undertaken.

Another aspect of proton relaxation in water, namely proton exchange processes, has also been studied by HP-NMR. Proton exchange between water molecules has been the subject of numerous investigations since the pioneering experiments of Meiboom et al. ${ }^{(498,499)} \mathrm{Spin}-\mathrm{spin}\left(T_{2}\right)$ relaxation, rotating frame $\left(T_{1 \rho}\right)$ relaxation and low frequency spin-lattice $\left(T_{1}\right)$ relaxation of protons ${ }^{(495.300-502)}$ as well as oxygen-17 linewidth ${ }^{(503-505)}$ and oxygen-17 scalar relaxation ${ }^{(506-507)}$ NMR studies have been performed; all but one, however, at ambient pressure only. Lamb et al. ${ }^{\text {(5os) }}$ studied the temperature $\left(0-100^{\circ} \mathrm{C}\right)$ and pressure $(0-560 \mathrm{MPa})$ dependence of proton lifetime $\tau_{e}$ in liquid water via the proton $T_{1 \rho}$ relaxation. $\tau_{\mathrm{e}}$ decreases linearly with temperature along the saturation vapour line (svp), though an Arrhenius dependence with an apparent activation energy $E=14.44(\mathrm{~kJ} / \mathrm{mol})$ may be fitted to the data within experimental error. At higher densities, however, plots of $-\log \tau_{\mathrm{e}}$ versus $T^{-1}$ at constant density are distinctly curved showing that a simple activated jump mechanism is inappropriate for describing the proton dynamics in water. A plot of the density dependence of $\tau_{c}$ at constant temperature is shown in Fig. 95.

A strong decrease of $\tau_{\mathrm{e}}$ with density is observed at high temperatures in contrast to other dynamical quantities in water which are less sensitive to density variations at high temperatures. At low temperatures the onset of an anomalous increase of $\tau_{\mathrm{e}}$ with density is indicated in accord with the behaviour of all dynamic properties. It should be mentioned also that the average exchange time $\tau_{\mathrm{e}}$ is not a fundamental quantity, hence it is less well suited to elucidate the actual mechanism of the proton transport in water. An excellent and thorough treatment of prototropic charge migration in water has been reported recently by Halle and Karlström. ${ }^{(506,507)}$

\subsection{Aqueous Solutions of Simple Hydrophobic Model Compounds}

Aqueous solutions of non-electrolytes constitute some of the most complex and least well understood liquids. Solutes with large apolar groups and a small polar group to enhance the solubility in water are well suited for studies of the dynamic implications of the so called hydrophobic hydration and hydrophobic interaction. Numerous NMR studies under ambient conditions have been reported $^{(509-512)}$ but only a few systems have been studied under high pressure conditions. These studies are of relevance in understanding the effect of pressure upon hydrophobic interactions. 
The latter are only barely understood and conflicting conclusions have been drawn in the literature..$^{(513,514)}$

6.4.1. Water-Dioxane Mixtures. The pressure and concentration dependences of the deuteron spinlattice relaxation times and the viscosity have been measured by Lee and Jonas ${ }^{(515)}$ in $\mathrm{D}_{2} \mathrm{O}$ dioxane and $\mathrm{H}_{2} \mathrm{O}$-dioxane- $\mathrm{d}_{8}$ mixtures at pressures up to $450 \mathrm{MPa}$ and at $303 \mathrm{~K}, 313 \mathrm{~K}$ and $323 \mathrm{~K}$. When plotted against concentration, many properties of water-dioxane mixtures pass through a maximum or minimum at low concentrations of dioxane. Hence it has been suggested that up to a mole fraction $X_{\mathrm{D}}=0.2$ dioxane promotes the water structure in a sense discussed by Frank and Evans. ${ }^{(516)}$ According to the latter authors non-electrolytes increase the water structure in their immediate environment by having clathrate cages formed around the hydrophobic parts. At higher mole fractions all experimental evidence indicates a disruption of the random hydrogen bond network in water. Since quadrupolar relaxation is the dominant relaxation mechanism for the deuteron relaxation in both $D_{2} \mathrm{O}$ and dioxane $\mathrm{d}_{8},{ }^{2} \mathrm{H}-T_{1}$ can provide direct information about the dynamic structure of these solutions. Lee and Jonas ${ }^{(515)}$ discussed their relaxation time measurements mainly in terms of hydrodynamic mode-coupling relations (eqns $(21,136)$ ) and the effect of pressure on the reorientation of water and dioxane molecules in a strongly disrupted H-bond network. Figure 96 compares the pressure dependence of the deuteron $T_{1}$ of $\mathrm{D}_{2} \mathrm{O}$ (Fig. 96a) and dioxane- $\mathrm{d}_{8}$ (Fig. 96b) at various mole fractions of $\mathrm{D}_{2} \mathrm{O}$.

Neat $\mathrm{D}_{2} \mathrm{O}$ at $303 \mathrm{~K}$ displays the unusual facilitation of reorientations upon initial compression and an almost pressure-independent relaxation time at $X_{\mathrm{w}}=0.95$ though the viscosity changes strongly $(\sim 40 \%)$ with pressure at the same concentration. At higher dioxane concentrations $\left(X_{w}<0.90\right)$ water behaves more like a simple molecular liquid in that $T_{1}$ decreases with increasing pressure. It was found that these solutions solidify upon compression. The phase transition is marked by a discontinuity in the pressure dependence of $T_{1}$. Also the latter increased with pressure in the solid phase as predicted from eqn (60) in the slow motion limit. The corresponding dioxane- $d_{8}$ relaxation times display a pressure dependence which is usually observed in molecular liquids except in solutions with a high water content where the pressure dependence is less pronounced. With increasing concentration the deuteron $T_{1}$ values exhibit a minimum at a mole fraction $X_{\mathrm{w}} \simeq 0.2-0.3$ indicating a slowing down of reorientational motions of water (and also dioxane) molecules upon addition of the solute molecules. This effect has been interpreted in terms of a water structure stabilization due to clathrate-like cage formation around the solutes. At high solute concentrations the $\mathrm{H}$-bond network is strongly disrupted, hence the lack of retarding $\mathrm{H}$-bond interactions, resulting in facilitated reorientations with increased solute concentration. Also the coupling of rotational and translational motions of the water molecules decreases with increasing density and the decrease is more pronounced the lower
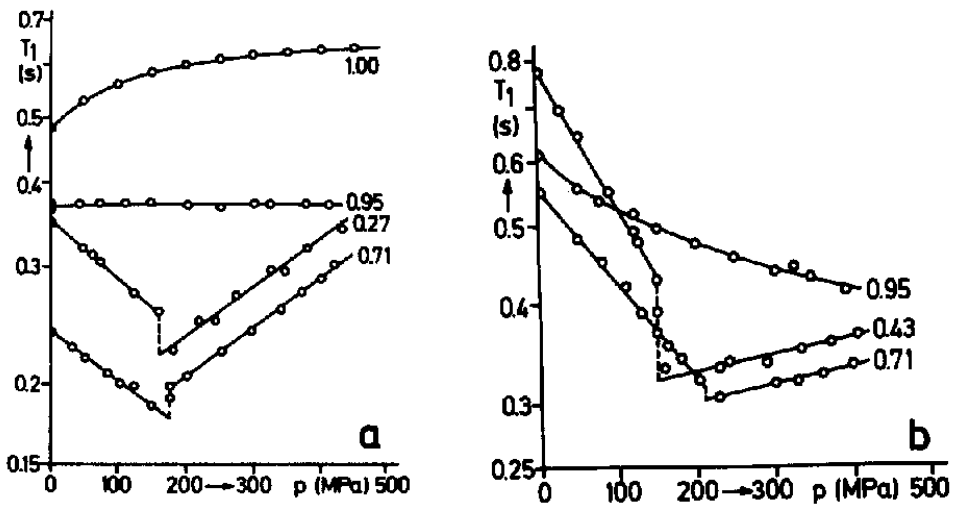

Fic. 96. Pressure dependence of the deuteron relaxation time $T_{1}$ of (a) $\mathrm{D}_{2} \mathrm{O}$ and (b) dioxane- $\mathrm{d}_{\mathrm{b}}$ in $\mathrm{D}_{2} \mathrm{O}-$-dioxane solutions at $303 \mathrm{~K}$ and different mole fractions of $\mathrm{D}_{2} \mathrm{O}$.(51s) 
the water content of the solutions. Under ambient pressure, however, the RT-coupling parameter $\kappa$ (eqn (136)) does not change with concentration $(\kappa=1)$, hence Debye's equation can be applied to relate orientational correlation times and the viscosities. With respect to the dioxane- $d_{8}$ molecules, $\kappa \leqslant 0.15$ has been found, which is in accord with results obtained in other simple molecular liquids.

6.4.2. Water-Dimethylsulphoxide (DMSO) Mixtures. The binary system dimethylsulphoxide (DMSO)-water has often been regarded as a model system to study hydrophobic hydration and interaction phenomena. The use of DMSO as a cryo-protective agent for the prevention of damage to living cells at low temperatures is well known. ${ }^{(517)}$ Many physical properties of DMSO-water mixtures exhibit extremes as a function of solute concentration at about a mole fraction $X_{\mathrm{DMSO}}=0.35$ corresponding to a composition $R(=$ moles water $/ \mathrm{mol} \mathrm{DMSO}) \simeq 2$. At this composition molecular mobility is at a minimum and DMSO-water interactions are at a maximum due to the formation of $\mathrm{Me}_{2} \mathrm{SO}-\mathrm{H}_{2} \mathrm{O}$ hydrogen bonds. At concentrations $X_{\text {DMso }} \leqslant 0.1$ corresponding to $R \geqslant 9$ the effect of DMSO on the dynamic structure of liquid water is in dispute. The indications are, however, that hydrophobic hydration with its concomitant slowing down of the mobility of the hydration water molecules is a characteristic feature in these dilute solutions. This behaviour should not be interpreted, however, as producing a cooperative ordering of water molecules, thereby increasing the long-range stucture. $^{(518-519)}$ Several NMR studies of the DMSO-water system at ambient pressure are reported in the literature and are discussed in a recent review by Lang and Lüdemann. ${ }^{(18)}$ The only high pressure relaxation study has been performed on DMSO-water mixtures at $25^{\circ} \mathrm{C}$ by Baker and Jonas. ${ }^{(519)}$ They studied the pressure dependence of the deuterium $T_{1}$ in DMSO- $\mathrm{D}_{2} \mathrm{O}$ mixtures and the proton $T_{1}$ and $\mathrm{H}_{2} \mathrm{O}$ self-diffusion coefficients in DMSO- $\mathrm{d}_{6}-\mathrm{H}_{2} \mathrm{O}$ mixtures. Figure 97 a gives their result for the deuterium spin-lattice relaxation times $T_{1}$ which monitor only reorientational motions of the water molecules, and Fig. 97b shows the water self-diffusion coefficients, which reflect their translational mobility.

As DMSO is added to water, the values of $T_{1}$ and $D$ decrease. The rotational and translational motions of water molecules slow down progressively with increasing DMSO concentration. The most notable feature is that the initial increase with pressure of the values of $T_{1}$ and the self-diffusion coefficients for the proton and deuterons, observed for pure water, is maintained in solutions up to $X_{\text {DMSo }}=0.1$, corresponding to a water-DMSO ratio $R \simeq 9$. The persistence of this anomaly in the dilute DMSO solutions indicates that the random $\mathrm{H}$-bond network is not broken up until at least $X_{\text {DMSO }} \simeq 0.1$. This implies, of course, that the effect of dissolved DMSO upon the dynamic structure of the $\mathrm{H}$-bond network cannot extend beyond the immediate neighbourhood of the solute. The anomalous pressure effect is only small, of course, as the random network is already strongly
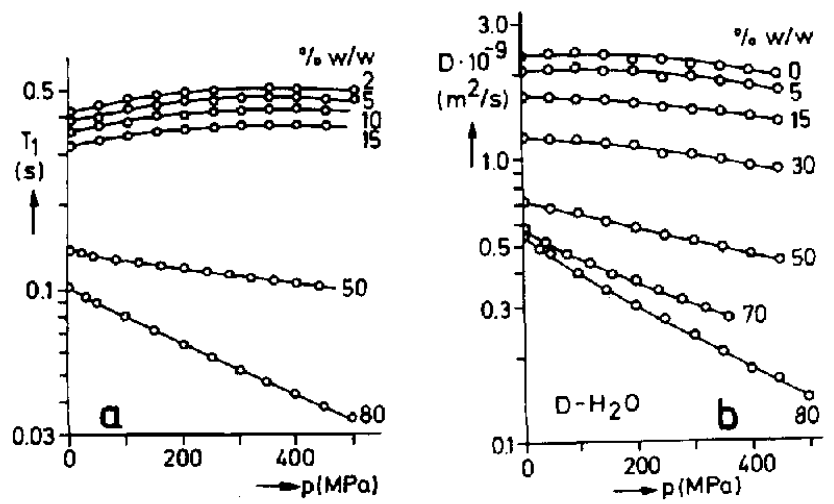

FIG. 97. (a) Pressure dependence of the water deuteron relaxation time $T_{1}$ in $\mathrm{DMSO} / \mathrm{D}_{2} \mathrm{O}$ solutions at different weight percent of DMSO. ${ }^{(519)}$ (b) Pressure dependence of the water self-diffusion coefficient $D$ in $D^{2}$ MSO- $d_{6} / \mathrm{D}_{2} \mathrm{O}$ solutions at different weight percent of DMSO. ${ }^{(519)}$ 
perturbed by thermal excitations at room temperature. This insensitivity to pressure of a strongly perturbed $\mathrm{H}$-bond network is another puzzling feature of aqueous solutions and is quite different from other molecular liquids. At high DMSO concentrations a strong pressure dependence is observed accordingly.

6.4.3. Alcohol-Water Mixtures. Another class of effectively hydrophobic solutes are the lower alcohols, which are sufficiently soluble in water to be studied over an appreciable concentration range. Though studied extensively at ambient pressure, high pressure investigations are scarce. Woolf ${ }^{(520)}$ has recently discussed the pressure dependence of transport properties in methanol-water mixtures. The diffusion of alcohol molecules is enhanced upon initial compression ${ }^{(521)}$ and the enhancement becomes stronger with decreasing temperature as is shown in Fig. 98. It has been suggested that lower alcohols enhance the open network structure of water, ${ }^{(522.523)}$ and Angell ${ }^{(524)}$ has proposed that in alcohol-water systems the alcohol molecules are located in the most open, highly structured patches within the H-bond network, and these are most affected by an increase in pressure. Accordingly, Easteal and Woolf ${ }^{(521)}$ proposed that at low temperatures and pressures, addition of methanol to water up to about $30 \mathrm{~mol} \%$ should cause a modification of the $\mathrm{H}$-bond network by accomodation of the alcohol inside cavities accompanied by formation of water-methanol hydrogen bonds. The effect of pressure was considered to cause disruption or distortion of those bonds and hence to enhance the translational mobility.

The system $t$-butanol/water is another well studied solution of an effectively hydrophobic solute. It

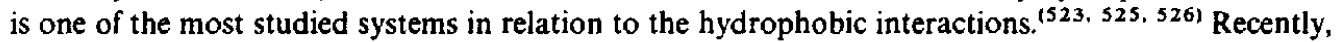
the first high pressure NMR study on this system was performed in our laboratory by Woznyj. ${ }^{(527)}$ Spin-lattice relaxation times of deuterium $\left({ }^{2} \mathrm{H}\right)$, oxygen-1 7 and protons $\left({ }^{1} \mathrm{H}\right)$, as well as self-diffusion coefficients of the protons, were studied over the whole composition range at temperatures up to $430 \mathrm{~K}$ and pressures up to $200 \mathrm{MPa}$. The observed deuterium relaxation rate is a mole fraction weighted average of the water rate and the hydroxyl-group of the alcohol. Additional ${ }^{17} \mathrm{O}-T_{1}$ of enriched water allowed the determination of the water ${ }^{2} \mathrm{H}-T_{1}$ over the whole concentration range, monitoring the rotational motions of water molecules in these solutions. At low solute concentrations $\left(X_{\mathrm{t}-\mathrm{Bu}} \leqslant 0.1, R=9\right.$ ) and low temperatures the rotational mobility of the water molecules slows down strongly upon the addition of $t$-butanol. This may be ascribed to the retarding effect of hydrophobic hydration and the disappearance of bulk water at a solute mole-fraction $X_{\mathrm{t}-\mathrm{Bu}} \simeq 0.1$ corresponding to the composition of the clathrate hydrate. At intermediate concentrations the decrease in $T_{1}$ levels off and only at high solute concentrations is a further decrease of $T_{1}$ observed. The effect of pressure upon

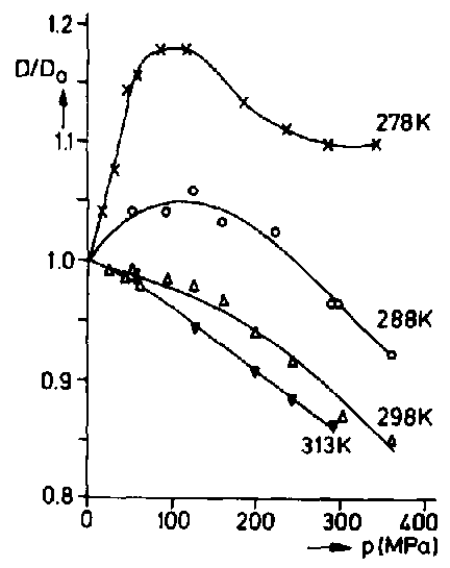

FIG. 98. Isothermal pressure dependence of the normalized self-diffusion coefficient of methanol dissolved in water. ${ }^{(521)}$ 
the water mobility in a network which is perturbed by the solute is illustrated in Fig. 99. The anomalous increase in mobility upon compression diminishes with increasing solute concentration and reverts to normal behaviour at a mole fraction of the solute $X_{t-B u} \simeq 0.07$. At this composition only water molecules coordinated directly to the $t$-butanol molecules are left. This observation again demonstrates that the solute molecules do not influence more than the first shell of the hydration water dynamically. The dynamic behaviour of the solute was also studied thoroughly. As an example, Fig. 100a shows the concentration and pressure dependence of the deuterium- $T_{1}$ of the methyl-groups of the $t$-butanol molecule. At high temperatures the reorientation, which is a composite of internal and overall tumbling motion, is almost independent of concentration and only slightly retarded by compression. In contrast, at low temperatures the relaxation rate increases with concentration with the slope of $\mathrm{d} R_{1} / \mathrm{d} c$ increasing strongly with rising pressure and decreasing temperatures. This is an indication of hydrophobic hydration structures around the apolar $\mathrm{CH}_{3}$-groups which are most stable at low temperatures and are then most sensitive to the destructive action of hydrostatic pressure.

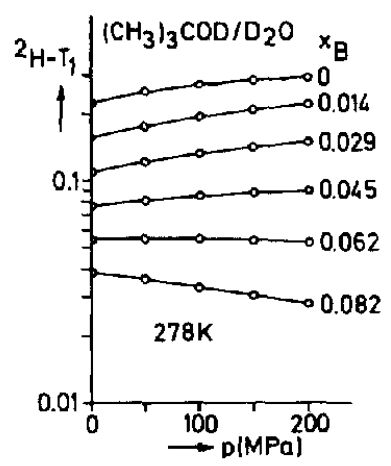

FIG. 99. Pressure dependence of the water deuteron relaxation time $T_{1}$ in $t$-butanol/ $\mathrm{D}_{2} \mathrm{O}$ mixtures at different mole-fractions $x_{B}$ of $t$-butanol. ${ }^{(527)}$
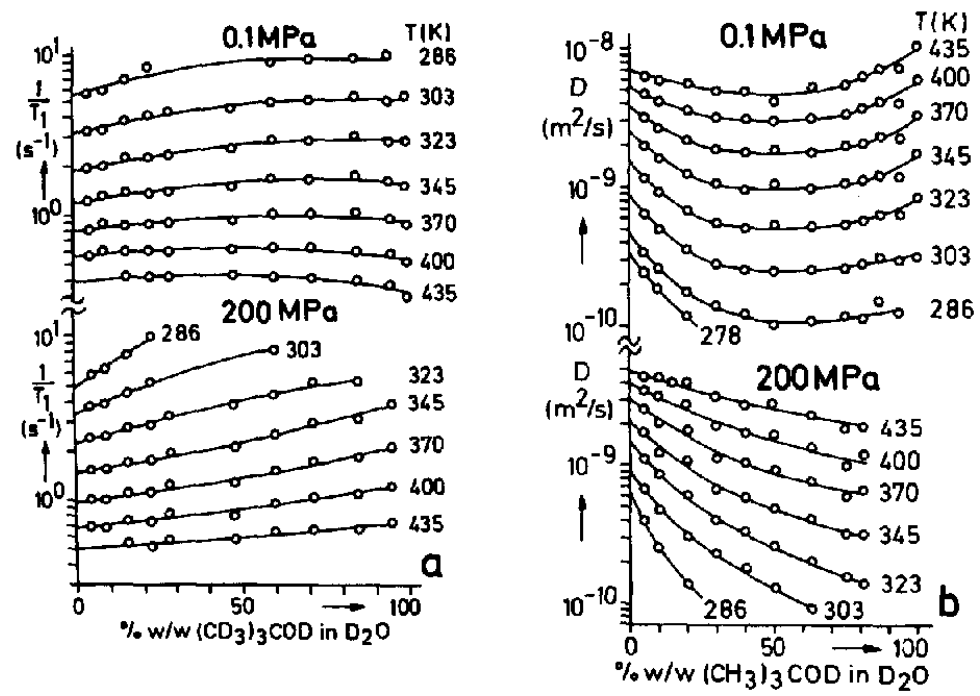

FIG. 100. Concentration and temperature dependence of (a) the methyl deuteron relaxation rate (1/ $\left.T_{1}\right)$ and (b) the self-diffusion coefficient $D$ of $t$-butanol in $t-\mathrm{BuOH} / \mathrm{D}_{2} \mathrm{O}$ mixture. ${ }^{(527)}$ 
Figure $100 \mathrm{~b}$ shows the pressure and concentration dependence of the self-diffusion coefficient $D$ of the $t$-butanol molecules. Under ambient pressure conditions the translational mobility decreases with increasing solute concentration up to a composition $R \simeq 2$, and increases slightly at still higher concentrations. The initial decrease is most pronounced at low temperatures. At high pressure, $D$ decreases at all temperatures with increasing solute concentration. Again, the slope $\mathrm{d} D / \mathrm{d} c$ increases with decreasing temperature. These characteristics are in accord with the view that hydrostatic pressure as well as solutes disturb the well-connected $\mathrm{H}$-bond network at low temperatures thereby enhancing all molecular motions.

\subsection{Aqueous Solutions of Simple Ionic Solutes}

Like hydrostatic pressure, dissolved ions may be considered network-breaking agents. which disrupt the transient $\mathrm{H}$-bond connectivity patterns. Whereas hydrostatic pressure causes a global distortion, ions perturb the $\mathrm{H}$-bond network only locally. Electrostatic interactions lead to rather specific relative orientations of coordinated water molecules. These local arrangements show characteristic differences between cations and anions which have been elucidated in recent years with neutron scattering ${ }^{(528)}$ and computer simulation ${ }^{(529)}$ techniques. Typically, water molecules close to cations always orient their hydrogen atoms away from the cation, with the dipole moment vector being tilted relative to the radial direction. The tilt angle depends on the charge density of the ion as well as on the anion and on the total concentration. With anions water molecules almost always orient one of the $\mathrm{OH}$-bonds in a nearly linear fashion towards the anion. The sharpness of these local structural features depends, of course, on the strength of the ion-dipole interactions, hence on the charge density, and on the amount of thermal energy stored in the motional degrees of freedom.

At temperatures above room temperature thermal excitations cause a high degree of distortion of the H-bond network. The influence of weak interactions like hydrogen bonds and/or ion-dipole forces is not especially pronounced. In undercooled solutions, however, thermal distortions of the H-bond network may be strongly reduced leading to pronounced effects of other network breaking agents like hydrostatic pressure and/or dissolved ions. ${ }^{\left({ }^{(50)}\right.}$ Undercooling thus permits a thorough study of the influence of ion-dipole and $\mathrm{H}$-bonding interactions upon structure and dynamics of aqueous electrolyte solutions. One of the major advantages of the application of hydrostatic pressure is a continuous change in the state of the H-bond network at constant (low) thermal excitation. If one starts with a well developed network, high pressure transforms the latter into a strongly distorted state with greatly weakened or even disrupted H-bonding interactions. Hence liquid water under high pressure loses its characteristic tetrahedral connectivity patterns rendering the state of the liquid similar to a polyalcohol. Starting with an already thermally disrupted network, pressure may not be expected to exhibit pronounced changes in the dynamic structure of the network, hence in any of its physical properties.

Aqueous electrolyte solutions have been studied with NMR techniques under ambient pressure and temperature conditions almost since the invention of this powerful technique. There are several excellent reviews ${ }^{\mathbf{( 5 3 0 - 5 3 3 )}}$ available dealing with NMR relaxation experiments under ambient condjtions. Simple electrolytes have been classified as 'structure-making' or 'structure-breaking' ions depending on whether they enhance or reduce the average mobility of water molecules in these solutions. However, these definitions depend strongly on the state of the H-bond network, hence on temperature and pressure, as will be shown below. From infinite dilution extrapolations relative molecular mobilities of hydration water molecules can be deduced at ambient conditions. ${ }^{(530)}$ Ions with a large charge/radius ratio retard the motions of coordinated water molecules strongly, conversely a small charge density speeds up the fluctuations of hydration waters. It also became obvious that orientational fluctuations of coordinated water molecules cannot be isotropic, ${ }^{(530)}$ though a suggested reorientation around a radially oriented dipole moment vector is not in accord with known structural features of the coordination shell. An attempt has also been made to infer structural characteristics like the relative orientation of hydration water molecules from dipolar relaxation measurements. Though the pair distribution function cannot be obtained, several trial functions can be tested. ${ }^{(534,535)}$ It turns out that the local structure is less well defined for ions of low charge density, 
hence coordinated water molecules experience a considerable motional freedom in accord with dynamic results.

Finally, these studies demonstrated convincingly that chemical exchange processes of hydration water molecules occur on time-scales largely comparable to the time scales of orientational and positional fluctuations. Hence, the life-time of hydration waters is in most cases only slightly longer than corresponding correlation times. ${ }^{(536)}$ High pressure NMR studies of molecular motions in aqueous ionic solutions are very rare, probably because of the modest pressure effects upon transport and relaxation coefficients that are generally observed in a highly distorted H-bond network above room temperature. This situation will change greatly at low temperatures as was demonstrated recently and this topic will be discussed at length below.

6.5.1. Self-Diffusion in Aqueous Solutions of Atomic Ions. Akai and Jonas ${ }^{(537)}$ performed NMR spin-echo experiments to study the pressure dependence up to $450 \mathrm{MPa}$ of the self-diffusion coefficient $D$ of heavy water at three temperatures $(303 \mathrm{~K}, 333 \mathrm{~K}, 363 \mathrm{~K})$ in concentrated $\mathrm{LiCl} / \mathrm{D}_{2} \mathrm{O}(4.5 \mathrm{M})$, $\mathrm{CaCl}_{2} / \mathrm{D}_{2} \mathrm{O}(3.06 \mathrm{M})$ and $\mathrm{CsCl} / \mathrm{D}_{2} \mathrm{O}(4.5 \mathrm{M}, 7 \mathrm{M})$ solutions. Shear viscosities and densities were also obtained for these systems. The diffusion coefficient ratios $D / D_{0}$, with $D_{0}$ the corresponding neat water value, are $<1$ for $\mathrm{LiCl}$ and $\mathrm{CaCl}_{2}$ and $\approx 1$ for $\mathrm{CsCl}$, with no obvious trend with temperature and pressure. This has been interpreted in terms of structure formation by the ions $\mathrm{Li}^{+}<\mathrm{Ca}^{2+}$ and structure breaking behaviour in the case of $\mathrm{Cs}^{+}$. It is claimed that at high temperatures, $\mathrm{Cs}^{+}$can also become a structure former. An inverse relation is obeyed for the self-diffusion coefficient and the viscosity in accord with the Stokes-Einstein relation

$$
D=k T / C \pi \eta r,
$$

with $r=0.138 \mathrm{~nm}$ the radius of the water molecule, and with a coefficient $C \simeq 5$, just between the slip $(C=4)$ and stick $(C=6)$ limiting values.

It is worth mentioning that the Stokes-Einstein relation is not obeyed at low temperatures in the system $\mathrm{Ca}\left(\mathrm{NO}_{3}\right)_{2} / \mathrm{H}_{2} \mathrm{O}{ }^{\left({ }^{(538)}\right.}$ where self-diffusion, viscosity and conductivity show different glass transition temperatures $T_{0}$ in corresponding VTF-descriptions. Assuming an Arrhenius temperature dependence $D(T)$, which is only approximately valid, apparent activation energies $E_{\mathrm{p}}$ and $E_{\mathrm{v}}$ at constant pressure and constant volume, respectively, have been deduced. The latter are roughly equal, in sharp contrast to non-associated liquids, where $E_{\mathrm{P}} \simeq 2 E_{\mathrm{V}}$ is often found. The self-diffusion is relatively insensitive to pressure in all systems studied, exhibiting a slight decrease with increasing pressure above room temperature. This is in striking contrast to molecular liquids where self-diffusion is a very sensitive function of pressure. ${ }^{(227,228)}$ At $303 \mathrm{~K}$ a weak maximum in $D(p)$ is seen indicating an increasing mobility upon initial compression even at these high concentrations. Accurate selfdiffusion coefficients of several alkali metal ions in aqueous solutions became available only recently from NMR spin-echo techniques. ${ }^{(539)}$ From tracer diffusion ${ }^{(540)}$ and conductivity ${ }^{(541)}$ data it is known that ions diffuse much slower than water molecules even at very high concentrations. This indicates an independent diffusion of the water molecules, hence no long-lived hydration sphere. It may be hoped that pressure NMR experiments will provide ionic diffusion coefficients under pressure in the near future.

6.5.2. Spin-Relaxation in Aqueous Solutions of Atomic lons. Apart from self-diffusion, Lee and Jonas $^{(542)}$ and Lee et al. ${ }^{(543)}$ studied the pressure dependence of the proton $\left({ }^{1} \mathrm{H}\right)$ and deuteron $\left({ }^{2} \mathrm{H}\right)$ spin-lattice relaxation in concentrated $(4.5 \mathrm{M})$ aqueous $\mathrm{LaCl}_{3}, \mathrm{CaCl}_{2}, \mathrm{LiCl}, \mathrm{KBr}, \mathrm{RbBr}$ and $\mathrm{CsBr}$ solutions at $303 \mathrm{~K}$. Both the ${ }^{1} \mathrm{H}$ and ${ }^{2} \mathrm{H}$ relaxation rate is enhanced in the $\mathrm{LaCl}_{3}, \mathrm{CaCl}_{2}$ and $\mathrm{LiCl}$ solutions relative to pure water. This has been said to be due to a structure forming ability of the cations. Dissolved $\mathrm{KBr}, \mathrm{RbBr}$ and $\mathrm{CsBr}$ decrease both relaxation rates slightly which has been interpreted as being due to the structure breaking ability of the cations mainly. With increasing pressure the deuteron relaxation rate, hence the average reorientational correlation time (see eqns ( 55 and 56)) of the 'structure-breaking' salts $\mathrm{KBr}, \mathrm{RbBr}$ and $\mathrm{CsBr}$ exhibit a minimum at about $200 \mathrm{MPa}$; it decreases in $\mathrm{LiCl}$ solutions and increases in $\mathrm{CaCl}_{2}$ and $\mathrm{LaCl}_{3}$ solutions. The viscosity increases 
steadily with pressure in these solutions indicating an increase of the Debye reorientational correlation time

$$
\tau_{\mathrm{D}}=\frac{\eta}{k T} V_{\text {eff }}=\frac{4 \pi}{3} r^{3} \frac{k \eta}{k T}
$$

( $\kappa$ measures the degree of coupling between rotation and translation).

Hence either the effective hydrodynamic volume $V_{\text {eff }}$ of the water molecules or the quadrupole coupling constant $\chi$ (eqn (58)) must change with pressure. It is interesting to note that the deuterium relaxation rate decreases monotonically with rising pressure in the presence of clay surfaces which are H-bond network structure breaking 'agents' ${ }^{(544)}$ Contrary to large ionic solutes, at clay surfaces reorientational motions of surface waters are retarded considerably upon compression.

Contrary to the ${ }^{2} \mathrm{H}-T_{1}$, the proton relaxation rates increase monotonically with pressure for the 'structure breaking' salts but exhibit a minimum for the 'structure forming' salts which shifts to lower pressures in the order $\mathrm{La}^{3+}<\mathrm{Ca}^{2+}<\mathrm{Li}^{+}$. Hence the pressure dependence of the proton relaxation rates is similar to the behaviour of the self-diffusion coefficient. A conclusive interpretation of these findings could not be given at that time. However, the results suggested that interesting new insights might be gained from a thorough study of the dynamic structure of aqueous electrolyte solutions at low temperatures and high pressures. Consequently, multinuclear relaxation experiments under pressure have been performed in undercooled aqueous electrolyte solutions in our laboratory over the last years. Results of these studies will be discussed below.

\subsubsection{Reorientational Motions of Water Molecules in Undercooled lonic Solutions. Orientational} fluctuations of water molecules in various metastable electrolyte solutions have been investigated by measuring deuterium spin-lattice relaxation rates $R_{1}\left({ }^{2} \mathrm{H}\right){ }^{(545-552)}$ Using emulsions and applying hydrostatic pressure enabled us to reach the dispersion regime $(\omega \tau \simeq 1)$ in all solutions for all concentrations, and hence to acquire detailed information about the dynamic structure of these systems with relaxation experiments. In the fast motion regime $(\omega \tau \ll 1)$, deuterium relaxation rates reflect only the average rotational mobility of water molecules. The effect of ions and pressure on the latter will be discussed first.

At low temperatures the rapid completion of the H-bond network hinders molecular motions rather strongly. Ionic solutes and/or hydrostatic pressure disturb the $\mathrm{H}$-bond network and cause an increase in molecular mobility. To illustrate this point, Fig. 101 compares the pressure and concentration dependence of a low temperature $(T=238 \mathrm{~K})^{2} \mathrm{H}-T_{1}$ isotherm of $\mathrm{NaCl}$ and $\mathrm{KCl}$ dissolved in $\mathrm{D}_{2} \mathrm{O}$. Whereas $\mathrm{Na}^{+}$-ions are generally considered as structure forming, $\mathrm{K}^{+}$-ions are considered to be structure breakers. A behaviour similar to $\mathrm{Na}^{+}$has been observed with dissolved $\mathrm{Li}^{+}, \mathrm{Mg}^{2+}, \mathrm{Ca}^{2+}$, $\mathrm{F}^{-}$; also $\mathrm{Cs}^{+}$-ions cause similar effects as $\mathrm{K}^{+}$-ions.

Increasing pressure causes a dramatic increase in the rotational mobility $\left(\sim T_{1}\left({ }^{2} \mathrm{H}\right)\right)$ in neat water. ${ }^{(475)}$ With dissolved ions the effect becomes less pronounced with increasing solute concentration. A qualitatively different behaviour emerges from a comparison of different ions. Weakly hydrating ions $\left(\mathrm{K}^{+}, \mathrm{Cs}^{+}, \mathrm{Cl}^{-}, \mathrm{Br}^{-}, \mathrm{I}^{-}\right)$always increase further the rotational mobility by simply disrupting the $\mathrm{H}$-bond network without interacting strongly with water molecules. Because of small charge densities of these solutes, the resulting ion-dipole forces are insufficient to hinder reorientations of coordinated water molecules. They are, however, strong enough to induce a preferred orientation during the mean residence time $\tau_{\mathrm{ex}}$ of hydration waters, causing a structural mismatch in the well-developed $\mathrm{H}$-bond network. This leads to a weakening of $\mathrm{H}$-bonds to surrounding bulk water molecules, hence to less hindered fluctuations in the neighbourhood of these ions.

Both network breaking agents, dissolved ions and hydrostatic pressure, are seen to act in parallel and cause similar effects. This has been noted earlier by Kanno and Angell. ${ }^{(553)}$ They studied the pressure and concentration dependence of the homogeneous nucleation temperature $T_{\mathrm{H}}$ in simple ionic solutions and noted a similar depression of $T_{\mathbf{H}}$ with either pressure or ionic solutes. This is interesting in view of the scaling behaviour of transport and relaxation coefficients in metastable water (eqn (129)), with $T_{\mathrm{S}}(p)$ following $T_{\mathrm{H}}(p)$ closely. Assuming $T_{\mathrm{S}}(c)$ to follow $T_{\mathrm{H}}(c)$ in an analogous fashion offers a simple quantitative explanation for the observed similarity of both pressure and concentration 

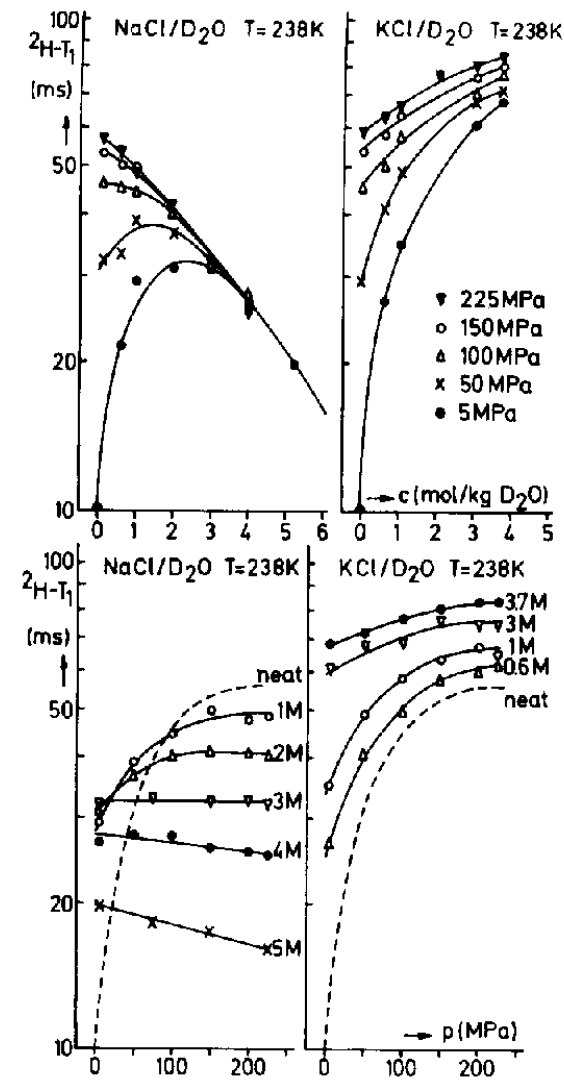

FIG. 101. Pressure and concentration dependence of the water deuteron relaxation time $T_{1}$ in undercooled $\mathrm{NaCl} / \mathrm{D}_{2} \mathrm{O}$ and $\mathrm{KCl} / \mathrm{D}_{2} \mathrm{O}$ solutions. Concentrations are given on the molality scale $\left(\mathrm{mol}\right.$ salt $\left./ \mathrm{kg} \mathrm{D}_{2} \mathrm{O}\right){ }^{(3 \$ 1)}$

effects upon dynamic properties. ${ }^{(552)}$ Both effects level off at compositions $R\left(=\operatorname{mol} \mathrm{D}_{2} \mathrm{O} / \mathrm{mol}\right.$ salt $)$ where only hydration water is present in the solution. This demonstrates clearly that these anomalous effects must be intimately related to the presence of a well-connected $\mathrm{H}$-bond network.

Strongly hydrating ions like $\mathrm{Li}^{+}, \mathrm{Na}^{+}, \mathrm{F}^{-}$etc. show a more complex behaviour. At low hydrostatic pressure the $\mathrm{H}$-bond network is well developed and all simple ions, whether strongly or weakly hydrating, disrupt the network and increase the average molecular mobility. This effect reaches a maximum at concentrations where only water molecules hydrating the ions are left. These water molecules are unable to form connected H-bond patterns. In all known cases the concentrations of the maximum effect correspond to the edge of the glass-forming composition range. ${ }^{(541,554-560)}$ In the absence of pronounced $\mathrm{H}$-bonding interactions, ion-dipole forces retard reorientations at higher concentrations.

Increasing hydrostatic pressure changes the well-connected $\mathrm{H}$-bond network continuously towards a strongly perturbed state with distorted and weakened $\mathrm{H}$-bonds. In this state ion-dipole interactions dominate over $\mathrm{H}$-bonding interactions and strongly hydrating ions cause a continuous slowing down of the average mobility of water molecules with increasing solute concentration. This sort of behaviour, misleadingly attributed to a structure making ability of these ions, is generally observed in a thermally disrupted $\mathrm{H}$-bond network above room temperature. Also the reorientational mobility ceased to increase upon compression whenever a composition was reached where, after accounting for proper coordination numbers of the hydration shells of the strongly hydrating cations, insufficient water molecules are left to form large enough domains with undistorted $\mathrm{H}$-bond interactions which 
could cause a sizeable pressure effect. An important conclusion drawn from these considerations is the notion that water bridging must become important in these highly concentrated solutions, i.e. the anions are coordinated on average to the hydration spheres of the cations and are not hydrated separately by the few bulk water molecules left. ${ }^{(549.350,361)}$ At still higher concentrations the relaxation rates are largely insensitive to pressure up to $250 \mathrm{MPa}$. Hence compression does not seem to influence much the dynamic properties of the hydration waters of strongly hydrated cations.

In all solutions of alkali and alkali earth halides investigated so far, cations exert a much more pronounced influence on the average mobility of water molecules than do the halide anions except for the fluoride anion $\mathbf{F}^{-}$. This is illustrated in Fig. 102.

Very low temperatures can be reached with a strongly perturbed H-bond network, brought about either by high pressure or high solute concentration, relaxation rates may then be followed beyond their maximum $(\omega \tau \simeq 1)$ into the slow motion regime $(\omega \tau>1)$. Only a few earlier studies took advantage of the glass forming tendency of highly concentrated ionic solutions to conduct relaxation experiments at low temperatures, though at ambient pressure only. Most of them are concerned with lithium halide solutions and have been discussed in connection with multinuclear relaxation studies in a recent review by Lang and Lüdemann. ${ }^{(181)}$

Using aqueous electrolyte emulsions under a pressure of $225 \mathrm{MPa}$ we were able to study the deuterium relaxation dispersion over the whole concentration range. These relaxation time curves $T_{1}(T, p, \omega, c)$ were interpreted with the simple two-site, two-mode approximation. ${ }^{(546)}$ Figure 103 shows the deuterium relaxation time curves of the hydration waters of $\mathrm{Li}^{+}$and illustrates characteristic features of $T_{1}(T, p, \omega, c)$ which were observed in all systems studied. There is a pronounced asymmetry of the relaxation time curve in the modified Arrhenius plot, where the temperature is scaled by the ideal glass transition temperature $T_{\mathrm{o}}$ of the solution. The relaxation time follows a VTF-behaviour over most of the temperature range. Within the motional model proposed this is due to cooperative tumbling and exchange processes which dominate the reorientational motions of the hydration water molecules. In the slow motions regime a much weaker temperature dependence becomes obvious. Note that the relaxation time curve would be symmetric about the minimum if only a single mode with a VTF temperature dependence sufficed to account for the relaxation behaviour. ${ }^{(432)}$ To grasp the essential physics in accord with the local structure of the hydration shell, an anisotropic local mode was considered responsible for the deviations observed. As a thermally

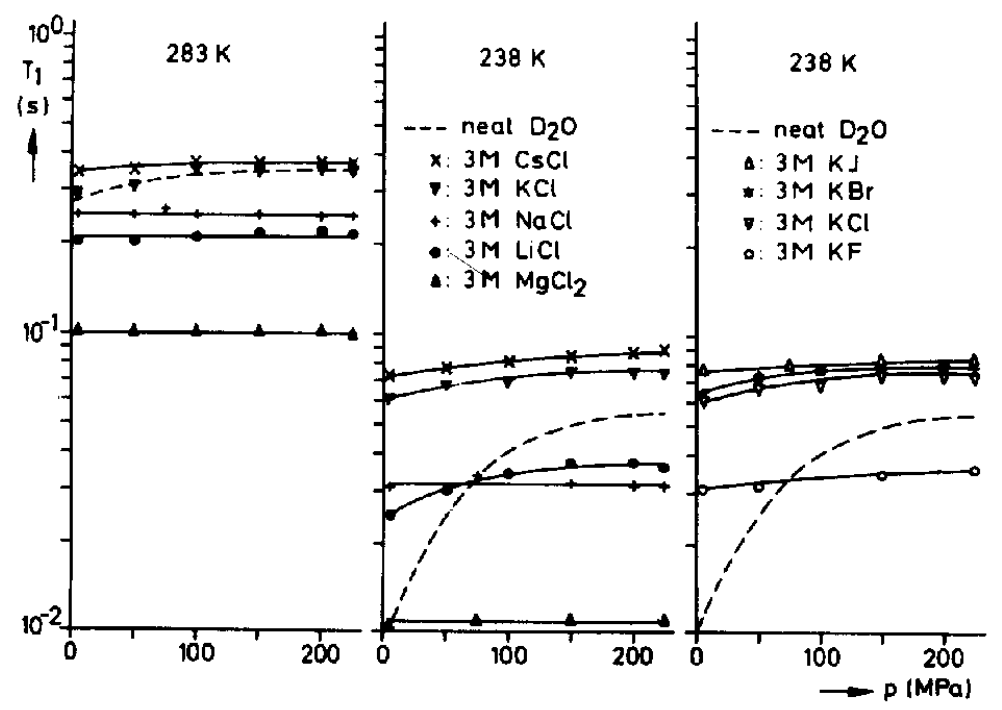

FIG. 102. Pressure dependence of the water deuteron relaxation time $T_{1}$ in undercooled electrolyte solutions at constant concentration and temperature. ${ }^{(591)}$ 


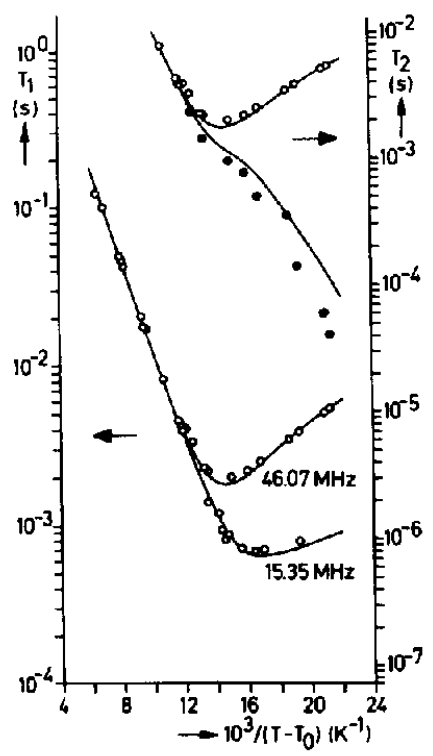

FrG. 103. Modified Arrhenius diagram of the water deuteron $(\bullet)$ spin-lattice $T_{1}$ and $(O)$ spin-spin $T_{2}$ relaxation times in an $11 \mathrm{M} \mathrm{LiCl} / \mathrm{D}_{2} \mathrm{O}$ solution $\left(T_{\mathrm{v}}=137 \mathrm{~K}\right)$. $^{(351)}$

activated process, its concomitant Arrhenius temperature dependence provides a sufficiently slow variation with temperature of the anisotropic mode for the latter to become the faster mode at low temperatures, hence to dominate the relaxation. The cooperative modes become too slow to provide an efficient relaxation channel in the slow motion regime.

Two other relaxation studies on this system are reported in the literature. ${ }^{(562-564)}$ Harmon and Sutter ${ }^{(563,564)}$ studied the ${ }^{2} \mathrm{H},{ }^{1} \mathrm{H},{ }^{7} \mathrm{Li}$ relaxation times over the same temperature range. They interpreted their data assuming an anisotropic internal reorientation around the molecular symmetry axis within the hydration complex. This local arrangement is not in accord with the available structural information. Furthermore, they considered a rigid hydration complex and assumed the complex reorientation to be infinitely slow. The depth of the deuterium relaxation time curve at the minimum is not in accord with the frequency dependence $T_{1}\left(T_{\min } \omega\right)$ observed in the other studies. ${ }^{(546,547,562)}$ This is reflected also in the unrealistically low value of the deuterium quadrupole coupling constant deduced. Boden and Mortimer ${ }^{(561,562)}$ accounted for the asymmetry of the relaxation time curve in terms of a Cole-Davidson distribution of correlation times and assumed a VTF temperature dependence of the average correlation time. Their VTF parameters are in reasonably good agreement with ours indicating that they are not very sensitive to the spectral density function used. In fact both approaches give an equally good representation of the experimental deuterium relaxation time curves though the frequency dependence of the spectral density functions is quite different in the slow motions regime.

Unfortunately, due to spectrometer limitations, deuterium relaxation times cannot be obtained at sufficiently low temperatures to resolve this issue. An interesting remark concerning the model dependence of an estimation of the deuterium quadrupole coupling constant $\left\langle\chi\left({ }^{2} \mathrm{H}\right)\right\rangle$ from the relaxation rate maxima may be made. If the deuterium relaxation rates $R_{1}\left({ }^{2} \mathrm{H}\right)$ obtained in neat metastable water and in an $11 \mathrm{M} \mathrm{LiCl} / \mathrm{D}_{2} \mathrm{O}$ solution are both fitted to a Cole-Davidson distribution, then $\left\langle\chi\left({ }^{2} \mathrm{H}\right)\right\rangle$ turns out to be larger in the ionic solution than in pure $\mathrm{D}_{2} \mathrm{O}$. This is in contradiction to all ab initio calculations which show that $\mathrm{Li}^{+}$lowers $\chi\left({ }^{2} \mathrm{H}\right)$. The following parameters for insertion 
into eqn (60), eqn (121) and eqn (128) have been obtained:

$\begin{array}{lcclll} & \chi\left({ }^{2} \mathrm{H}\right)(\mathrm{kHz}) & \tau_{0}(\mathbf{s}) & B(\mathrm{~K}) & T_{\mathrm{o}}(\mathrm{K}) & \beta_{\mathrm{CD}} \\ \mathrm{D}_{2} \mathrm{O} & 222 & 3.58 \times 10^{-14} & 687.6 & 133.6 & 0.58 \\ 11 \mathrm{M} \mathrm{LiCl} / \mathrm{D}_{2} \mathrm{O} & 264 & 8.22 \times 10^{-14} & 687.6 & 137 & 0.25 .\end{array}$

In more dilute solutions a simple two-site approximation has been proposed from solid state NMR data on the corresponding glasses. ${ }^{(561,562)}$ These authors concluded that the random networks of these glasses consist basically of $\mathrm{Li}^{+}\left(\mathrm{H}_{2} \mathrm{O}\right)_{4} \mathrm{Cl}^{-}$clusters with the excess water incorporated interstitially. They suggested that the average local structure in the metastable liquid state of aqueous $\mathrm{LiCl} \cdot \mathrm{RD}_{2} \mathrm{O}$ solutions in this composition range $(R=2-10)$ is closely related to the local structure of the glassy state. Following this suggestion we calculated the deuterium relaxation time curves $T_{1}(T, R, \omega)_{225 \mathrm{MPa}}$ for all compositions $R>N=4$ with

$$
R_{1}(T, R, \omega)=(N / R)\left(R_{1}\right)_{\text {hyd }}+(1-N / R)\left(R_{1}\right)_{\text {bulk. }} .
$$

The hydration water relaxation rate $\left(R_{1}\right)_{\text {hyd }}$ corresponds to the data obtained in the $11 \mathrm{M}$ solution and the bulk relaxation rate $\left(R_{1}\right)_{\text {bulk }}$ corresponds to the rate obtained in neat water under a pressure of $225 \mathrm{MPa}$. Because bulk and hydration water are in dynamic equilibrium both rates have to be taken at the same reduced temperature $\left(T-T_{0}(R)\right)$, with $T_{0}(R)$ the appropriate ideal glass transition temperature for the solution of composition $R$. The latter can be obtained from the experimental relaxation time curves noting that the composition dependence of the minimum temperature $T_{\min }$ of the $T_{1}(T)$ curve parallels that for known calorimetric glass transitions $T_{\mathrm{z}}(R)$. This is not surprising since at the minimum the correlation time $\tau_{2}(T)$ for the cooperative tumbling motions has to have the value $\omega \tau_{2}\left(T_{\min }\right) \simeq 1$ and $\tau_{2}(T)$ is given by the VTF equation. ${ }^{(545)}$

This approach has been applied to interpret the high pressure deuterium relaxation time isobars in all systems investigated. Figure 104 gives an illustrative example showing the relaxation contributions from the two sites, bulk and cationic hydration water, in the case of a $3 \mathrm{M} \mathrm{NaCl} / \mathrm{D}_{2} \mathrm{O}$ solution corresponding to a composition $R \simeq 17$. The ideal glass transition temperature $T_{0}(R)$ deduced from these investigations are compiled in Fig. 105.

According to eqn (128) an increase in $T_{0}$ upon addition of salt reflects a slowing down of cooperative tumbling modes of water molecules in these solutions, whereas a decreasing $T_{\mathrm{o}}$ indicates increasing molecular mobility. Hence, in a strongly distorted $\mathrm{H}$-bond network with weak $\mathrm{H}$-bonding interactions, strongly hydrating ions slow down molecular tumbling motions, whereas weakly

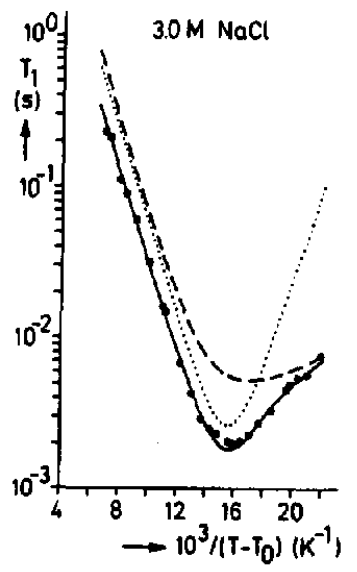

FIG. 104. Modified Arrhenius diagram of the total water deuteron relaxation time $T_{1}$ isobar (225 MPa) as well as the relaxation times corresponding to the bulk sites (...) and to the hydration sites (---) of the water molecules. ${ }^{(548)}$ 


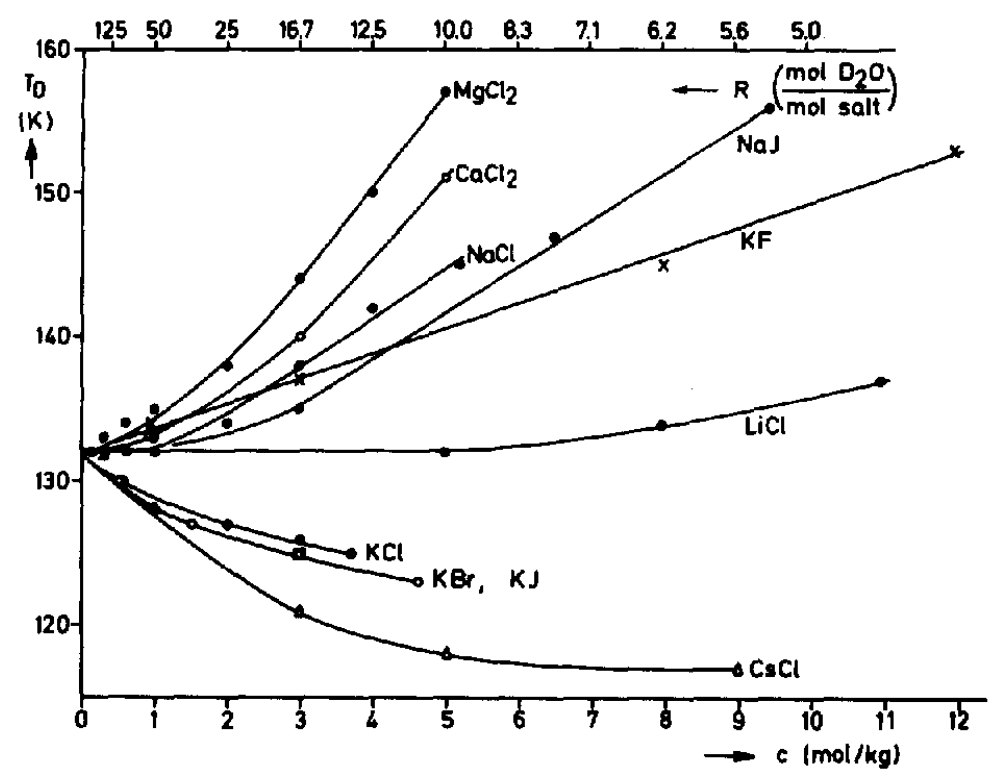

FIG. 105. Concentration dependence of the ideal glass transition temperatures $T_{0}$ of various metastable electrolyte solutions under high pressure $(p=225 \mathrm{MPa}) .^{(551)}$

hydrating ions facilitate diffusional processes, as do all simple ions dissolved in a well-connected $\mathrm{H}$-bond network above its bond percolation threshold. Again, cations exerts a much stronger effect upon the glass transition temperature than do anions except for the $\mathrm{F}^{-}$-anion. These results suggests that the influence of ionic solutes upon the temperature dependence of orientational and positional fluctuations is determined mainly by their effect on $T_{0}$.

Besides the deuterium relaxation rates, which monitor only reorientational motions of water molecules, the relaxation rates of the protons and of dissolved ions have been studied in metastable $\mathrm{LiCl}$ and $\mathrm{KF}$ solutions. These studies should provide a consistency test of the above mentioned motional model and should explore possible non-exponential relaxations at low temperatures as well as local structural correlations within the coordination sphere of the fluoride anion.

6.5.4. Multinuclear Spin-Relaxation in Undercooled LiCl-and KF-Solutions. Close to their respective glass transition regimes, dense metastable liquids often exhibit a non-exponential relaxation of structural fluctuations emerging either from a hierarchy of relaxation processes or from a superposition of exponentially relaxing processes, implying many parallel relaxation paths. ${ }^{(565)}$

Aqueous $\mathrm{LiCl}$ solutions provide the opportunity to study the dynamic behaviour of undercooled liquids which range from being very difficult to vitrify to being excellent glass formers by increasing the solute concentration. ${ }^{(566)}$ In an $11 \mathrm{M}$ solution, all water molecules may be considered to belong to the hydration sphere of the $\mathrm{Li}^{+}$cations. These solutions vitrify easily at low cooling rates hence emulsification is unnecessary. Deuteron $\left({ }^{2} \mathrm{H}\right)$, proton $\left({ }^{1} \mathrm{H}\right)$ and lithium $\left({ }^{6} \mathrm{Li},{ }^{7} \mathrm{Li}\right)$ relaxation rates as well as ${ }^{1} \mathrm{H}$ and ${ }^{7} \mathrm{Li}$ self-diffusion coefficients were followed to very low temperatures close to the calorimetric glass transition temperatures. ${ }^{(546,547)}$ These data were interpreted within the motional model discussed above. The model parameters were adjusted to the deuterium relaxation rate curves which are independent of pressure at these high concentrations. The corresponding relaxation rates of the protons $\left({ }^{1} \mathrm{H}\right)$ and lithium-6 $\left({ }^{6} \mathrm{Li}\right)$ nuclei, obtained at the same field $B_{0}=7.05$ Tesla, were calculated to be in fair agreement with experiment, as is shown in Fig. 106.

Furthermore, the relaxation time curves of ${ }^{7} \mathrm{Li}$ in $\mathrm{D}_{2} \mathrm{O}$ and $\mathrm{H}_{2} \mathrm{O}$ become identical if proper allowance is made for the dynamic isotope effect, i.e. the glass transition temperature $T_{0}$ must be 


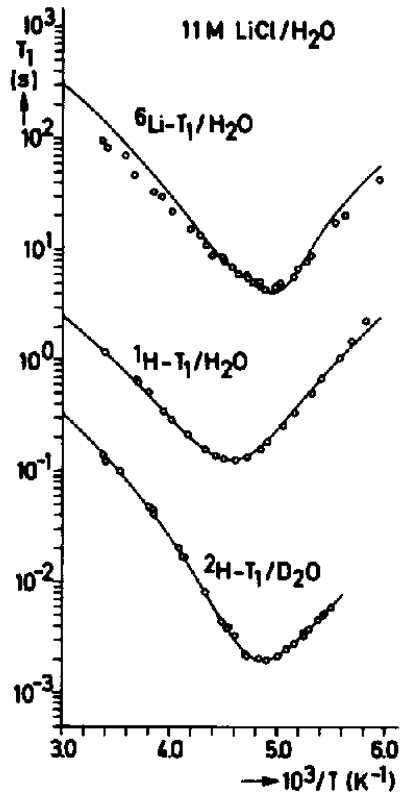

FIG. 106. Comparison of deuteron $\left({ }^{2} \mathrm{H}\right)$, proton $\left({ }^{1} \mathrm{H}\right)$ and lithium- $6\left({ }^{6} \mathrm{Li}\right)$ spin-lattice relaxation time curves in metastable $11 \mathrm{M} \mathrm{LiCl} / \mathrm{D}_{2} \mathrm{O}$ solutions. ${ }^{(\$ 4)}$

shifted by $4 \mathrm{~K}$ in going from $\mathrm{D}_{2} \mathrm{O}$ to $\mathrm{H}_{2} \mathrm{O}$. It is the absence of any dipolar contribution to the ${ }^{7} \mathrm{Li}$ relaxation rate which renders the latter larger in $\mathrm{D}_{2} \mathrm{O}$ than in $\mathrm{H}_{2} \mathrm{O}$ due to the dynamic isotope effect. This proves directly that the electric field gradient at the $\mathrm{Li}^{+}$ion is due to fluctuations of the surrounding water dipoles.

The interpretation of the proton, deuteron and lithium- 6 data tacitly assumed exponentially decaying time correlation functions of the fluctuating variables (see eqn (37)). Proton relaxation data sampled at another field ( $B_{0}=2.35$ Tesla) together with ${ }^{1} \mathrm{H}$ data from Harmon and Sutter at $B_{0}=0.17$ Tesla, revealed deviations from the $\omega^{-2}$-Debye dependence of the corresponding spectral density functions. ${ }^{6} \mathrm{Li}$ and ${ }^{2} \mathrm{H}$ relaxation rate curves show no signs of non-exponential relaxation. ${ }^{(551.552)}$ The proton relaxation rates can be well represented, however, with a Cole-Davidson distribution (eqn (119)) of tumbling correlation times, or equivalently with a Kohlrausch law (eqn (138)) for the relevant time correlation function

$$
G(t)=G(0) \exp \left[-(t / \tau)^{\beta}\right],
$$

indicating the onset of nonexponential relaxation close to the glass transition temperature $T_{\mathrm{g}}{ }^{18}$

Aqueous KF solutions are of interest because the fluoride anion has a stronger interaction with water, hence its hydration shell is better defined than that of the potassium cation. The relative orientation of the water molecules coordinated to the $\mathrm{F}^{-}$anion has been discussed several times in the literature $^{(567-571)}$ and conflicting results have been obtained. As both the proton and the fluorine nuclei interact via magnetic dipole interactions, their relaxation rate depends sensitively on the ${ }^{1} \mathrm{H}-{ }^{19} \mathrm{~F}$ distance, and hence on the mean orientation of the water molecules in the hydration sphere.

Aqueous $\mathrm{KF}$ solutions vitrify less easily than aqueous $\mathrm{LiCl}$ solutions therefore emulsified samples and high pressure ( $p=225 \mathrm{MPa}$ ) had to be used to reach the dispersion region in these solutions. Deuteron, proton and fluorine-19 relaxation rates have been reported as function of pressure $(p \leqslant 250 \mathrm{MPa})$, temperature $(T \geqslant 170 \mathrm{~K})$ and concentration ( $c \leqslant 12$ molal) in light and heavy water. ${ }^{(551,552,572)}$ The $12 \mathrm{M}$ solution was considered to contain only water molecules hydrating the fluoride anions. The relaxation rates were interpreted within the two-mode two-site approximation 
with the model parameters adjusted to the deuterium relaxation rates of the $12 \mathrm{M}$ solutions. An almost linear orientation of the water molecules relative to the $\mathrm{F}^{-}$anion has been assumed. The resulting ${ }^{2} \mathrm{H}$ relaxation rate curve is, however, not very sensitive to the average orientation. Thus, deuteron relaxation rate curves cannot distinguish between a linear and an almost symmetric arrangement. The relaxation of the protons is governed by dipolar interactions. Within the coordination sphere these couplings are modulated by reorientational fluctuations of the intramolecular ${ }^{1} \mathrm{H}-{ }^{1} \mathrm{H}$ vectors, and intermolecular ${ }^{1} \mathrm{H}-{ }^{19} \mathrm{~F}$ vectors. Positional fluctuations also modulate the dipolar interaction with protons outside the coordination sphere. All structural parameters were taken from computer simulation results. ${ }^{(571)}$ Calculations have been performed for a linear and a symmetric ion-water arrangement changing only the ${ }^{1} \mathrm{H}-{ }^{19} \mathrm{~F}$ distances and the amplitude factors $\left|D_{\mathrm{mo}}^{(2)}\left(\Omega_{\mathrm{DP}}\right)\right|^{2}$. The resulting relaxation rate curves do not differ much due to the dominance of the ${ }^{1} \mathrm{H}-{ }^{1} \mathrm{H}$ contribution, but the results favour the linear configuration. Within the two-site approximation the relaxation rate curves $R_{1}\left({ }^{1} \mathrm{H}\right)$ for the more dilute solutions can be reproduced satisfactorily. ${ }^{(572)}$

The ${ }^{19} \mathrm{~F}$ nucleus is relaxed mainly via magnetic dipole couplings to the protons of the hydration water. This coupling is modulated by reorientational fluctuations of the ${ }^{19} \mathrm{~F}-{ }^{1} \mathrm{H}$ vectors and depends sensitively on the mean ${ }^{19} \mathrm{~F}-{ }^{1} \mathrm{H}$ distance. As only heteronuclear couplings contribute, the total ${ }^{19} \mathrm{~F}$ relaxation is a much more sensitive probe for the average local orientation of hydration waters than is the proton relaxation. A translational contribution due to couplings to protons outside the coordination sphere was also taken into account. The total relaxation rate was calculated for the linear and symmetric arrangement. The results clearly favour the linear configuration (Fig. 107). Within a twosite approximation the relaxation rates corresponding to more dilute solutions can also be reproduced fairly well.

The conclusion was that, at least in a highly distorted network the mean orientation of water molecules coordinated to $\mathrm{F}^{-}$-anions corresponds to an almost linear configuration in accordance with theoretical predictions.

\subsection{Aqueous Solutions of Hydrophobic Ions}

Aqueous solutions of tetraalkylammonium (TAA) ions are model systems used to investigate the effect of different modes of hydration (coulombic versus hydrophobic) upon the dynamic structure of

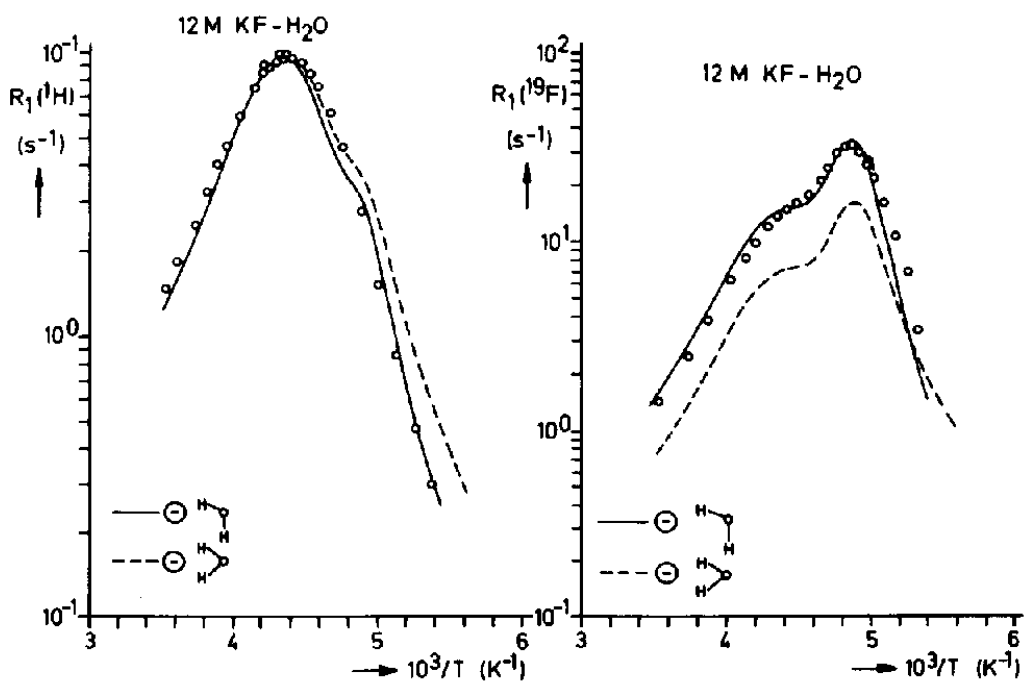

Fig. 107. Comparison of the proton $\left({ }^{1} \mathrm{H}\right)$ and fluorine-19 $\left({ }^{19} \mathrm{~F}\right)$ relaxation rate $R_{1}$ curves in $12 \mathrm{M} \mathrm{KF} / \mathrm{H} \mathrm{H}_{2} \mathrm{O}$ solutions under high pressure (225 MPa). The curves drawn were calculated within the two-mode two-site approximation and correspond to an almost linear $(--)$ and a symmetric $(-)$ anion-water arrangement. ${ }^{(\$ 72)}$ 
the H-bond network and the internal flexibility of the solute molecules. With increasing length of the alkyl chains the charge density decreases, diminishing the importance of ion-dipole interactions, and the apolar surface increases, promoting hydrophobic hydration structures in the immediate neighborhood. However, it is still unclear how long the alkyl chains have to be for the hydrophobic hydration phenomenon to dominate over ion-dipole forces in controlling the dynamic structure of the surrounding network. ${ }^{(573,574)}$ Furthermore, though the concept of hydrophobic hydration, i.e. the solvation of apolar groups with concomitant entropy reduction in the water, is widely used in biology and chemistry, the structural and dynamic consequences for the random, transient $\mathbf{H}$-bond network are still not well understood on a microscopic level.

Based on ideas dating back to Frank and Evans, ${ }^{(516)}$ and seemingly supported by many indirect thermodynamic and spectroscopic experiments, ${ }^{(575)}$ apolar solutes are believed to increase the amount of order and reduce the mobility of the hydration water. Recent direct structural investigations with neutron scattering techniques, however, do not show any pronounced ordering effects in the hydration shell of tetramethylammonium ions. ${ }^{(576)}$ Undercooled aqueous solutions of symmetric tetraalkylammonium ions may prove useful in this respect. Clathrate like hydrophobic hydration structures should be favoured by the network organization of cold water. In undercooled solutions these structures must become especially stable and long-lived due to reduced thermal excitations and strongly slowed down diffusional rearrangements of the transient $\mathrm{H}$-bond network. We therefore recently started multinuclear HP-NMR investigations of undercooled emulsions of aqueous solutions of tetraalkylammonium ions. ${ }^{(57)}$

6.6.1. Dynamics of the Water Molecules. Deuterium spin-lattice relaxation rates monitor orientational fluctuations of water molecules coordinated to tetraalkylammonium ions. In the case of dissolved tetramethylammonium $\left(\mathrm{TMA}^{+}\right)$ions, the latter show a temperature dependence which is neither observed in the case of strongly hydrating nor weakly hydrating atomic ions. If the $\mathrm{H}$-bond network is strongly perturbed because of thermal excitations these ions reduce the average mobility of water molecules. At low temperatures, however, H-bonds slow down reorientational fluctuations stronger than ion-water interactions. Accordingly, the average mobility of the hydration water molecules is enhanced compared to neat water as may be seen in Fig. 108.

This behaviour is also observed in solutions under high pressure, where strongly hydrating atomic ions reduce the mobility at all temperatures and weakly hydrating ions always increase the latter. Due

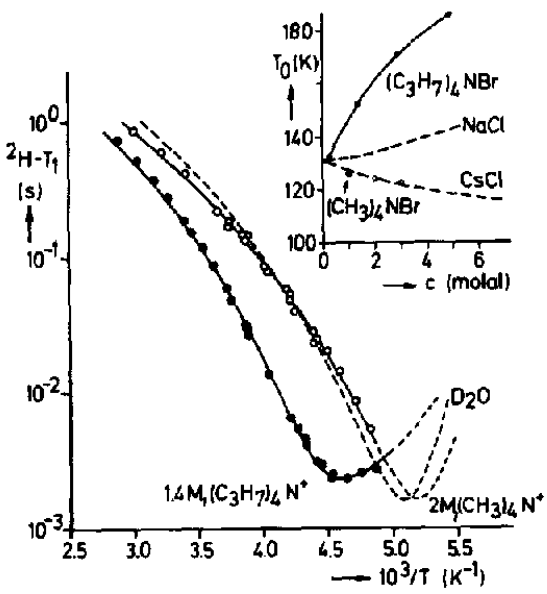

FIG. 108. Isobaric (225 MPa) temperature dependence of the hydration water deuteron relaxation time $T_{1}$ in an undercooled $2 \mathrm{M} \mathrm{TMABr} / \mathrm{D}_{2} \mathrm{O}$ solution and an undercooled $1.4 \mathrm{M} \mathrm{TPABr} / \mathrm{D}_{2} \mathrm{O}$ solution compared to undercooled $\mathrm{D}_{2} \mathrm{O}^{(580)}$ 


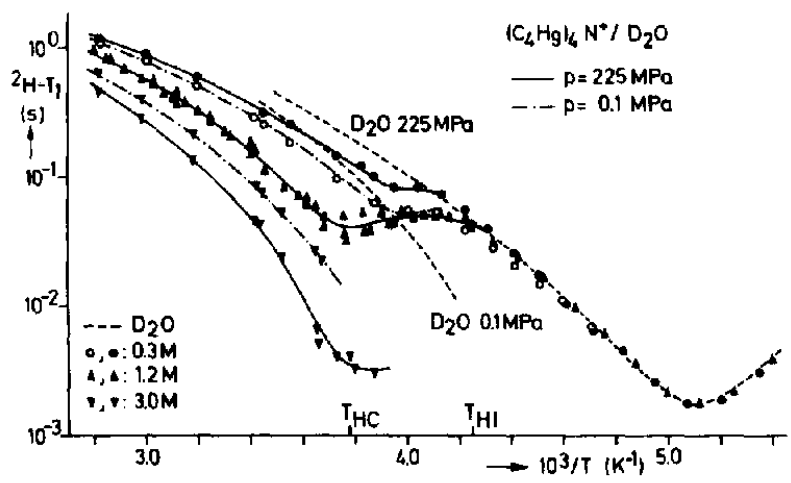

FIG. 109. Isobaric $(0.1 \mathrm{MPa}, 225 \mathrm{MPa})$ temperature dependence of the water deuteron relaxation times $T_{1}$ in $\mathrm{TBuABr} / \mathrm{D}_{2} \mathrm{O}$ solutions at various concentrations. $T_{\mathrm{HC}}$ and $T_{\mathrm{Hl}}$ correspond to the homogeneous nucleation temperatures of the $1.2 \mathrm{M}$ solution and neat water under ambient pressure. ${ }^{(380)}$

to the low charge density of $\mathrm{TMA}^{+}$ions, the reduced mobility can result only from increased $\mathrm{H}$-bond interactions, induced by the apolar surface, between the hydration water molecules. If $\mathrm{TMA}^{+}$ions were to induce clathrate-like transient structures, then one might expect a continued further reduction in the average mobility down to the lowest temperatures. The latter behaviour was indeed observed in the case of tetrapropylammonium (TPrA ${ }^{+}$) ions (Fig. 108). Both relaxation time curves can be fitted with spectral density functions corresponding to a Cole-Davidson distribution of correlation times (eqn (119)). The non-Arrhenius temperature dependence of the average correlation time was represented by a VTF-equation. The corresponding glass transition temperatures $T_{0}$ have been found to decrease with concentration in $\mathrm{TMA}^{+} / \mathrm{D}_{2} \mathrm{O}$ solutions but increase strongly in $\operatorname{TPrA}{ }^{+} / \mathrm{D}_{2} \mathrm{O}$ solutions. Contrary to these glass-forming systems, tetrabutylammonium $\left(\mathrm{TBuA}^{+}\right)$ions dissolved in water induce clathrate formation ${ }^{(578.579)}$ and do not form glasses. With increasing concentrations $\mathrm{TBuA}^{+}$-ions reduce the average mobility of water molecules strongly. Contrary to the aforementioned solutions a strong pressure effect has been observed in $\mathrm{TBuA}^{+} / \mathrm{D}_{2} \mathrm{O}$ solutions. Compression leads to facilitated motions at low concentrations $(0.3 \mathrm{M})$ but further reduces the average mobility at high concentrations $(3 \mathrm{M})$. The $1.2 \mathrm{M}$ concentration, which corresponds to a composition $R=46$ where only enough water molecules to hydrate the cations are left, does not show any pressure effect. At low temperatures, these solutions show a rather complex behaviour as may be seen in Fig. 109.(580)

6.6.2. Internal Flexibility of the $T A A^{+}$-Cations. Proton relaxation rates of the different methyleneand methyl groups of the alkyl chains in these ions have also been investigated. These rates mainly reflect internal reorientations about $\mathrm{C}-\mathrm{C}$ bonds as well as overall tumbling motions of the whole ions. In $\mathrm{TPrA}^{+}$and $\mathrm{TBuA}^{+}$solutions the relaxation rates decrease with increasing distance of the alkyl group from the central nitrogen atom. This, of course, reflects the increasing internal flexibility along the alkyl chain. In both systems the relaxation rates were followed into the slow motion regime. With increasing pressure and increasing concentration the relaxation rate maxima are shifted to higher temperatures, which, furthermore, are much higher than the corresponding temperatures of the relaxation rate maxima of the water molecules. This shows the strongly reduced segmental motions of the cations compared to the mobility of the surrounding water matrix. At low temperatures, strong spin diffusion causes an identical relaxation rate of all alkyl groups with the methyl groups acting as relaxation sinks, as is shown in Fig. 110. ${ }^{(580)}$

Furthermore, the methyl group reorientations provide a sensitive probe of the state of the surrounding $\mathrm{H}$-bond network. To a first approximation the slow overall tumbling mode does not contribute to the methyl group relaxation rate $R_{1}\left(\mathrm{CH}_{3}\right)$ which thus monitors only the fast anisotropic internal reorientation. This internal mode is slowed down considerably in $\operatorname{TPrA}^{+}$compared to 


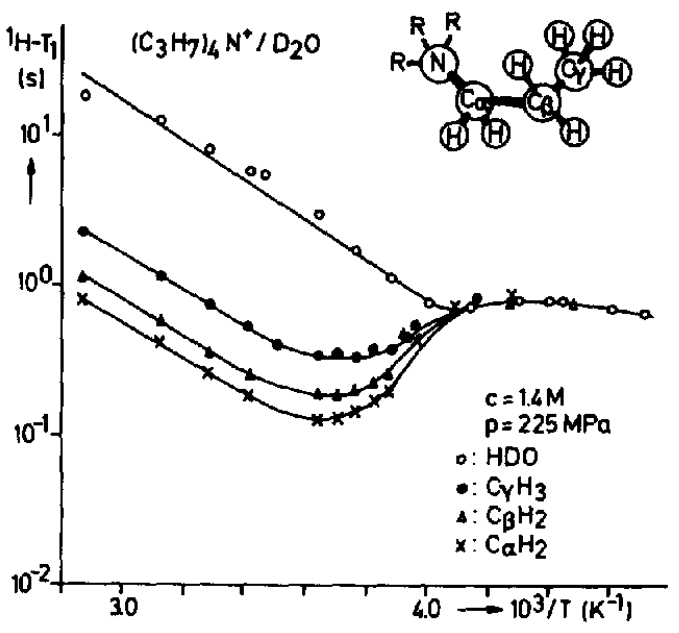

Fig. 110. Isobaric (225 MPa) temperature dependence of the proton relaxation times $T_{1}$ of the different alkyl groups of the cations in an undercooled $1.4 \mathrm{M} \operatorname{TPrABr} / \mathrm{D}_{2} \mathrm{O}$ solution. ${ }^{\left({ }^{(80)}\right)}$

$\mathrm{TMA}^{+} \mathrm{D}_{2} \mathrm{O}$ solutions. However facilitated reorientation is observed in $\mathrm{TBuA}^{+}$and this may reflect a change in the mode of hydration of these ions.

\section{CONCLUDING REMARKS}

The work reviewed here demonstrates that NMR relaxation measurements can yield detailed information about the molecular dynamics and local structure in liquids. In addition, conformational equilibria even for very complex molecules can often be derived from the analysis of coupling constants and chemical shifts. Dielectric relaxation and neutron scattering can provide similar and often complementary information. Compared to these methods, NMR relaxation methods have the advantage that the experiments are relatively simple. The small sample volumes required and the huge arsenal of modern NMR spectrometers supplies the experimentalist with very fine and variable tools, especially when extreme conditions are required. For the vast amount of data, obtained over a wide $T, p$ range, discussed in this review no real equivalent set has been obtained by neutron or dielectric methods.

One of the main limitations in the analysis of NMR relaxation is that for most low molecular weight liquids, experiments are confined to the extreme narrowing limit, and thus only integral correlation times can be derived. For water and aqueous solutions this limitation can be overcome by the combined application of low temperatures and high pressures. Spin-lattice and spin-spin relaxation times can be followed into the deeply supercooled range, where the measurements become frequency dependent.

For non-associating liquids, such as the alkanes and halomethanes, where all NMR data are collected in the extreme narrowing limit, only the application of pressure permits a wide temperature range of the substances to be studied at liquid densities and thus places severe constraints upon the motional models used to describe the dynamics.

Most of the liquid matter in the universe and also on our planet, occurs at high pressures and often also at extreme temperatures, and therefore a knowledge of the physical characteristics of liquids and liquid mixtures under these conditions is of great importance for the geosciences.

Many chemical and biotechnological processes use high pressure, and thus rely, for process optimization, on knowledge collected about liquid properties under these conditions. For simple liquids, molecular dynamics simulations can provide quantitative information, that for the time being 
still needs to be compared with experimental data. NMR relaxation measurements have in many cases provided data for this comparison and very often raised new questions, pointed out discrepancies and sometimes even decided which of the potentials used in the simulations yields the right answer and thus is the more realistic one.

Acknowledgements-Most of our results, cited in this section, were obtained by the students, working in our group during the last fifteeen years. Only their diligence and dedication made our contributions possible. In alphabetical order: Dr F. Bachl, S. Bradl, Dr W. Fink, U. Gaarz, Dr D. Girlich, A. Greiner-Schmid, Dr M. Has, Dr J. Hauer, N. Karger, Dr G. Klimke, Dr F. X. Prielmeier, Dr H. Radkowitsch, Dr T. Vardag, Dr G. Völkel, S. Wappmann, Dr M. Woznyj. We always could rely on the competent and fast technical support from our technicians and the faculty workshops. Especially R. Knott, D. Niesner, E. Treml and G. Wührl supported us often beyond the call of duty. Mrs I. Kulbartz typed and revised the many versions of this manuscript and Mrs A Birkmann and I. Cuno prepared the illustrations and strongly supported us in searches of the literature. We really needed all this help and highly appreciate it.

We gratefully acknowledge the generous financial support received from the Deutsche Forschungsgemeinschaft and the Fonds der Chemischen Industrie even in times of tight budgets.

\section{REFERENCES}

1. G. B. Benedex, Magnetic Resonance at High Pressures, Interscience, New York (1963).

2. J. JonAs, High Pressure Chemistry. NATO ASI 41, 65, Ed. H. Kelm, Reidel Dordrecht (1978).

3. W. L. EarL, H. VANNI and A. E. Merbach, J. Magn. Reson. 30, 571 (1978).

4. H. YAMADA, Rev. Sci. Instrum. 45, 640 (1974).

5. T. Asano and W. J. Le NoBle, Chem. Rev. 78, 407 (1978).

6. A. E. MeraAch, High Pressure Chemistry and Biochemistry. NATO ASI Ser. C 197, 311. Eds R. van Eldik and J. JoNAs. Reidel Dordrecht (1987).

7. H. Kanno, R. J. Speedy and C. A. ANGell, Science 189, 880 (1975).

8. C. A. ANGELL, and H. KANNO, Science 193, 1121 (1976).

9. W. F. Sherman and A. A. Stadtmuller, Experimental Techniques in High Pressure Research. Wiley, Chichester (1987).

10. J. Jonas and H. S. Gutowsky, Ann. Rev. Phys. Chem. 31, 1 (1980).

11. J. C. Jonas, High Pressure NMR, NMR Basic Principles and Progress, Vol. 24, Ed. J. Jonas, p. 85, Springer, Berlin (1991).

12. A. E. Merbach, Pure Appl. Chem. 54, 1479 (1982).

13. J. W. AKItT and A. E. Merbach, High Pressure NMR, NMR Basic Principles and Progress, Vol. 24, Ed. J. JonAS, p. 189, Springer, Berlin (1991).

14. H. Yamada, High Pressure NMR, NMR Basic Principles and Pragress, Vol. 24, Ed. J. Jonas, p. 233, Springer, Berlin (1991).

15. H. Yamada, M. Nakatsuka, H. Yamochi, M. Sawamura and A. Sera, Rev. Sci. Instrum. 62, 700 (1991).

16. H. Yamada, S. Nishitani and A. Sera, Chem. Lett. 6, 791 (1981).

17. U. GaArz and H.-D. LÜdemann, Ber Bunsenges. Phys. Chem. 80,607 (1975).

18. E.W. Lang and H.-D. Lüdemann, High Pressure NMR, NMR Basic Principles and Progress, Vol. 24, Ed. J. Jonas, p. 129, Springer, Berlin (1991).

19. U. Frey, L. Helm and A. E. Merbach, High Pressure Res. 2, 297 (1990).

20. F. BACHL and H.-D. LUdemanN, High Pressure Res. 6, 91 (1990).

21. T. Vardag, N. Karger and H.-D. Lüdemann, Ber. Bunsenges, Phys. Chem. 95, 859 (1991).

22. J. Paureau, Rev. Sci. Instr. 48, 1632 (1977).

23. A. Trokiner, N. Dahan, H. Theveneau and P. Papon, Rev. Sci. Instr. 55, 1616 (1984).

24. E. O. Stejskal and J. E. TAnner, J. Chem. Phys. 42, 288 (1965).

25. E. W. Lang and H.-D. LudemanN, Ber Bunsenges. Phys. Chem. 84, 462 (1980).

26. R. K. Williams, Rev. Sci. Instr. 49, 591 (1978).

27. H. S. V. TYReLl, and K. R. HARRIS, Diffusion in Liquids, Butterworth, London (1984).

28. P. STILBS, Prog. NMR Spectrosc. 19, 1 (1987).

29. J. Kärger, H. Pfeifer and W. Heink, Adr. Magn. Reson. 12, 1 (1988).

30. H. WeingärTner, Nuclear Magnetic Resonance (Specialist Periodical Report), Ed. G. A. WebB, The Chemical Society, London.

31. H. Y. Carr and E. M. Purcell, Phys. Rev. 94, 630 (1954).

32. E. L. Hahn, Phys. Rev. 80, 580 (1950).

33. D. M. Lamb, P. J. Grandinetii and J. Jonas, J. Magn. Reson. 72, 532 (1988).

34. E. O. STEJskal and J. E. TANNer, J. Chem. Phys. 42, 288 (1965).

35. H. Weingärtner, Z. Phys. Chem. NF 132, 129 (1982). 
36. W. D. Williams, E. F. W. Seymour and R. M. Cotts, J. Magn. Reson. 31, 271 (1978).

37. K. D. Hammonds and D. M. HeYeS, J. Chem. Soc. Faraday Trans. II, 84, 705 (1988).

38. D. M. HeYES, J. Chem. Soc. Faraday Trans. II, 83, 1985 (1987).

39. D. Chandler, J. D. Weeks and H. C. Andersen, Science 220, 787 (1988).

40. D. Chandler, J. Chem. Phys. 60, 3596 (1974).

41. N. F. Carnahan and K. E. Starling, J. Chem. Phys. 51, 635 (1969).

42. D. M. HeYes and L. V. WoODCOCK, Mol. Phys. 59, 1369 (1986).

43. R. J. SpeEdy, Mol. Phys. 62, 509 (1987).

44. D. Chandler, J. Chem. Phys. 62, 1358 (1975).

45. R. J. Speedy, F. X. Prielmeier, T. Vardag, E. W. Lang and H.-D. LüdemanN, Mol. Phys. 66, 577 (1989).

46. J. H. DYMOND, Physica 75, 100 (1974).

47. J. H. DYMOND, Chem. Phys. 17, 101 (1976).

48. J. A. Barker and D. Henderson, Rev. Mod. Phys. 48, 587 (1976).

49. B. J. Alder, D. M. Gass and T. E. Wainwright, J. Chem. Phys. 53, 3813 (1970).

50. T. Einwohner and B. J. Alder, J. Chem Phys. 49, 1458 (1968).

51. A. F. Collings and L. A. Woolf, J. Chem. Soc. Faraday Trans. I, 71, 2296 (1975).

52. S. Saeki, M. Tsubokawa, J. Yamanaka and T. Yamaguchi, Polymer 31, 2338 (1990).

53. K. R. Harris, H. N. Lam, E. Raedt and A. J. Easteal, Mol. Phys. 71, 1205 (1990).

54. B. Polzin and A. Weiss, Ber. Bunsenges. Phys. Chem. 94, 746 (1990).

55. A. K. Doolittle, J. Appl. Phys. 22, 1471 (19S1).

56. M. H. Cohen and J. Turnbull, J. Chem. Phys. 31, 1164 (1959).

57. D. L. Hogenboom, K. Krynicki and D. W. SAwyer, Mol. Phys. 40, 823 (1980).

58. E. W. LANG and H.-D. LÚdemanN, Angew. Chem. Int. Ed. Engl. 21, 315 (1982).

59. C. A. Angell, Water-A Comprehensive Treatise, Vol. 7, Ed. F. Franks, p. 1, Plenum Press, New York (1980).

60. E. W. LANG and H.-D. LÜDemann, Hydrogen-Bonded Liquids, NATO ASI Ser. C. Vol. 329, Eds. J. Dore and J. TEIXEIRA p. 333 (1991).

61. C. A. ANGell, J. Phys. Chem. 75, 3698 (1971).

62. H. E. Stanley and J. Teixeira, J. Chem. Phys. 73, 3404 (1980).

63. D. Bertolini, M. Cassettari, M. Ferrario, P. Grigolini and G. Salvetti, Adv. Chem. Phys. 62, 277 (1985).

64. D. Bertolini, M. Cassettari, M. Ferrario, P. Grigolini, G. Salvetti and A. Tani, J. Chem. Phys. 91,1179 (1989).

65. D. Bertolini, P. Grigolini and A. Tani, J. Chem. Phys. 91, 1191 (1989).

66. V. I. JASHKICHeV, Adv. Mol. Relax. Interact. Processes 24, 157 (1982).

67. S. Y. CHU and G. SPOsito, J. Chem. Phys. 69, 2539 (1978).

68. G. Sposito, J. Chem. Phys. 74, 6943 (1981).

69. G. W. Robinson, J. Lee, K. G. Casey and D. Statman, Chem. Phys. Lett. 123, 483 (1986).

70. G. W. Robinson, J. LeE and M.-P. BAsSeZ, J. Chem. Soc. Faraday Trans. II, 82, 2351 (1986).

71. G. W. Robinson, J. LeE and M.-P. Bassez, Chem. Phys. Lett. 137, 376 (1987).

72. M.-P. Bassez, J. LeE and G. W. Robinson, J. Phys. Chem. 91, 5818 (1987).

73. R. ZWANZIG and M. Bixon, Phys. Rev. A 2, 2005 (1970).

74. R. ZwanziG, J. Chem. Phys. 79, 4507 (1983).

75. R. Zwanzig and A. K. Harrison, J. Chem. Phys. 83, 5861 (1985).

76. J. T. Hrnes, Ann. Rev. Phys. Chem. 28, 301 (1977).

77. H. Vogel, Phys. Z. 22, 645 (1921).

78. G. S. Fulcher, J. Am. Chem. Soc. 77, 3701 (1925).

79. G. TAMmANN and W. Hesse, Z. Anorg. Chem. 156, 245 (1926).

80. F. Bloch, Phys. Rev. 70, 460 (1946).

81. N. Bloembergen, E. M. Purcele and R. V. Pound, Phys. Rev. 73, 679 (1948).

82. 1. Solomon, Phys. Rev. 99, 559 (1955).

83. A. Abragam, The Principles of Nuclear Magnetism, Clarendon Press, Oxford (1961).

84. C. P. Slichter, Principles of Magnetic Resonance, 2nd edn, Springer, Berlin (1980).

85. R. R. Ernst, G. Bodenhausen and A. WokAun, Principles of Nuclear Magnetic Resonance in One and Two Dimensions, Clarendon Press, Oxford (1987).

86. J. MC ConNelL, The Theory of Nuclear Magnetic Relaxation in Liquids, Cambridge University Press, Cambridge (1987)

87. J. KowALEWSKI, Ann. Rep. NMR Spectrosc. 22, 308 (1990).

88. J. KoWALEWSKI, Ann. Rep NMR Spectrosc. 23, 289 (1991).

89. R. K. WANGSNESS and F. BlOCH, Phys. Rev. 89, 728 (1953).

90. F. Bloch, Phys. Rev. 102, 104 (1956).

91. F. BloCH, Phys. Rev. 10S, 1206 (1957).

92. A. G. RedFIELD, Adv. Magn. Reson. 1, 1 (1965).

93. D. L. Cummins, G. B. Bacskay, N. S. Hush, S. Engström and B. Halle, J. Chem. Phys. 82, 2002 (1985).

94. E. W. Lang, H.-D. Lüdemann and L. Piculell, J. Chem. Phys. 81, 3820 (1984).

95. N. C. PYPer, Mol. Phys. 45, 441 (1982). 
96. E. R. Henry and A. Szabo, J. Chem. Phys. 82, 4753 (1985).

97. R. SJÖbLM and J. TEGENFELDT, J. Magn. Reson. 27, 405 (1977).

98. S. Szymanski, A. M. GryfF-Keller and G. BinsCH, J. Magn. Reson. 68, 399 (1986).

99. R. M. LyNDEN-BeLl, Mol. Phys. 22, 837 (1971).

100. R. KuBo, J. Math. Phys. 4, 174 (1963).

101. J. H. Freed, G. V. Bruno and C. F. Polnaszek, J. Phys. Chem. 75, 3385 (1971).

102. P.-O. Westlund, Nuclear Magn. Reson. 20, 452 (1991).

103. H. W. SPIess, NMR 15 - Basic Principles and Progress, Eds. P. Diehl, E. FluCK and R. Kosfeld, Springer, Berlin (1978).

104. P. S. Hubbard, Phys. Rev. 131, 275 (1963).

105. C. A. Sholl, J. Phys. C.: Solid State Phys. 14, 447 (1981).

106. H. C. TORreY, Phys. Rev. 92, 962 (1953).

107. L.-P. Hwang and J. H. FreEd, J. Chem. Phys. 63, 4017 (1975).

108. Y. Ayant, E. Belorizky, J. Alizon and J. Gallice, J. Physique 36, 991 (1975).

109. L. G. Werbelow, J. Magn. Reson. 57, 136 (1984).

110. L. G. Werbelow, J. Chem. Soc. Faraday Trans. II, 83, 897 (1987).

111. L. G. Werbelow and D. M. Grant, Adv. Magn. Reson. 9, 189 (1977),

112. R. L. Vold and R. R. Vold, Prog. NMR Spectrosc. 12, 79 (1978).

113. P. S. Hubbard, Phys. Rev. 109, 1153 (1958).

114. M. D. Zeidler, Ber Bunsenges. Phys. Chem. 72, 481 (1968).

115. A. D. Bain and R. M. LYNDEN-BeLl, Mol. Phys. 30, 325 (1975).

116. L. G. Werbelow and D. M. Grant, J. Chem. Phys. 63, 544 (1975).

117. L. G. Werbelow, D. A. Ikenberry and G. Pouzard, J. Magn. Reson. 51, 409 (1983).

118. L. G. Werbelow, J. Magn. Reson. 67, 66 (1986).

119. F. Winter, R. Kimmich, Mol. Phys. 45, 33 (1982).

120. M. T. Stokes and D. C. Allion, J. Chem. Phys. 70, 3572 (1979).

121. M. F. Rose, Elementary Theory of Angular Momentum, Wiley, New York (1957).

122. B. Halle and H. Wennerström, J. Chem. Phys. 75, 1928 (1981).

123. B. Halle, H. Wennerström and L. Piculell, J. Phys. Chem. 88, 2482 (1984)

124. P. S. Hubbard, J. Chem. Phys. 53, 985 (1970).

125. B. Halle and H. WenNerströM, J. Magn. Reson. 44, 89 (1981).

126. L. G. Werbelow, J. Chem. Phys. 70, 5381 (1979).

127. L. G. Werbelow and A. G. Marshal, J. Magn. Reson. 43, 443 (1981).

128. P.-O. Westlund and H. WenNerström, J. Magn. Reson. 50, 451 (1982).

129. G. C. Wick, Phys. Rev. 73, 51 (1948).

130. C. H. WANG, J. Magn. Reson. 9, 75 (1973).

131. D. H. LeE and R. E. D. MC Clung, Chem. Phys. 112, 23 (1987).

132. J.-Cl. Leicknam, Phys. Rev. A 22, 2286 (1980).

133. R. E. D. MC Clung, J. Chem. Phys. 73, 2435 (1980).

134. H. Hauer, E. W. Lang, and H.-D. Lüdemann, Chem. Phys. 62, 195 (1981).

135. C. Deverell, Mol. Phys. 18, 319 (1970).

136. P. S. Hubbard, Phys. Rev. A 6, 2421 (1972).

137. E. N. Ivanov, Soviet Physics JETP 18, 1041 (1964).

138. D. Chandler, J. Chem. Phys. 60, 3500 (1974).

139. J. O'Dell and B. J. Berne, J. Chem. Phys. 63, 2376 (1975).

140. D. Chandler, $J$. Chem. Phys. 60, 3508 (1974).

141. I. Solomon and N. Bloembergen, J. Chem. Phys. 25, 261 (1956).

142. J. S. BlichARSKI, Z. Naturforsch. 27A, 1355 (1972).

143. H. S. Gutowsky, D. M. MC CAll and C. P. Slichter, J. Chem. Phys. 21, 279 (1953).

144. H. S. Gutowsky and A. SalkA, J. Chem. Phys. 21, 1688 (1953).

145. H. S. GutowsKY and C. H. Holm, J. Chem. Phys. 25, 1228 (1956).

146. H. GutowsKY, Dynamic Nuclear Magnetic Resonance Spectroscopy, Eds. L. M. JACKMAN and F. A. CotTon, Academic Press, New York (1975).

147. J. Jonas, A. Allerhand and H. S. Gutowsky, J. Chem. Phys. 42, 3396 (1965).

148. R. Küо, J. Phys. Sac. Jpn 9, 935 (1954).

149. P. W. ANDERSSON, J. Phys. Soc. Jpn. 9, 316 (1954).

150. J. Kaplan, J. Chem. Phys. 28, 278 (1958).

151. J. Kaplan, J. Chem. Phys. 29, 462 (1958).

152. L. M. JACKMAN and F. A. CotTon, Eds., Dynamic Nuclear Magnetic Resonance Spectroscopy, Academic Press, New York (1975).

153. J. I. KaPlan and G. Fraenkel, NMR of Chemically Exchanging Systems, Academic Press, New York (1980).

154. J. SANDSTRÖM, Dynamic NMR Spectroscopy, Academic Press, London (1982).

155. H. A. Kramers, Physica 7, 284 (1940).

156. A. Nitzan, Adv. Chem. Phys. 70, 489 (1988). 
157. J. T. Hynes, Ann. Rev. Phys. Chem. 36, 573 (1985).

158. B. J. Berne, M. Barkovec and J. E. Straub, J. Phys. Chem. 92, 3711 (1988).

159. N. B. SLATER, Theory of Unimolecular Reactions, Cornell University Press, Ithaka (1959).

160. P. Pechukas, Dynamics of Molecular Collisions, Ed. W. H. Miller, Plenum Press, New York (1976).

161. D. F. CAlef and J. M. Deutch, Ann. Rev. Phys. Chem. 34, 493 (1983).

162. D. Chandler, J. Chem. Phys. 68, 2959 (1978).

163. J. L. SkINNER and P. G. WOLYNES, J. Chem. Phys. 69, 2143 (1978).

164. J. L. SKINNER and P. G. Wolynes, J. Chem. Phys. 72, 4913 (1980).

165. J. A. Montgomery, D. Chandler and B. J. Berne, J. Chem. Phys. 70, 4056 (1979).

166. J. A. Montgomery, S. L. Holmgren and D. Chandere, J. Chem. Phys. 73, 3688 (1980).

167. B. J. Berne, J. L. SKinner and P. G. Wolynes, J. Chem. Phys. 73, 4314 (1980).

168. J. Jonas and X. PenG, Ber. Bunsenges. Phys. Chem. 95, 243 (1991).

169. S. P. Velsko, H. Waldeck and G. R. Fleming, J. Chem. Phys. 78, 249 (1983).

170. C.-L. XIE, D. Camprell and J. Jonas, J. Chem. Phys. 92, 3736 (1990).

171. R. F. Grote and J. T. Hynes, J. Chem. Phys. 73, 2715 (1980).

172. R. F. Grote and J. T. HYNes, J. Chem. Phys. 74, 4465 (1981).

173. H. WenNerström, Mol. Phys. 24, 69 (1972).

174. B. Halle, Mol. Phys. 53, 1427 (1984).

175. H. M. MC Connell, J. Chem. Phys. 28, 430 (1958).

176. J. R. Zimmermann and W. E. Brittin, J. Phys. Chem. 61, 1328 (1957).

177. H. WinKLer and A. GuTSZE, Adv. Mol. Relax. Interact. Proc. 21, 159 (1981).

178. S. Forsen and R. A. Hoffmann, Acta Chem. Scand. 17, 1787 (1963).

179. S. Forsen and R. A. Hoffmann, J. Chem. Phys. 39, 2892 (1963).

180. S. Forsen and R. A. Hoffmann, $J$. Chem. Phys. 40,1189 (1964).

181. R. A. Hoffmann and S. Forsen, Prog. NMR Spectrose. 1, 16 (1966).

182. C. Deverel., R.E. Morgan and J. H. Strange, Mol. Phys. 18, 553 (1970).

183. U. Eliar, A. Baram and G. Nayon, J. Chem. Phys. 89, 5584 (1988).

184. D. Beckert and H. Pfeifer, Ann. Phys. 16, 262 (1965).

185. H. G. HerTZ, Ber. Bunsenges. Phys. Chem. 71, 979 (1967).

186. B. Halle, Mol. Phys. 56, 209 (1985).

187. B. Halle, Mol. Phys. 60, 319 (1987).

188. A. E. Merbach, Pure Appl. Chem. 59, 161 (1987).

189. Y. Ducommun, A. E. Merbach, Studies in Inorganic Chemistry 7. Inorganic High Pressure Chemistry, Ed. R. van EldiK, p. 69, Elsevier, Amsterdam (1986).

190. M. Mizuno, Sh. Funahashi, N. Nakasuka and M. Tanaka, Bull. Chem. Soc. Jpn. 64, 1988 (1991).

191. L. M. JACKMAN and F. A. Cotron, Eds., Dynamic Nuclear Magnetic Resonance Spectroscopy, Academic Press, New York (1975).

192. L. M. JaCKMAN, Dynamic Nuclear Magnetic Resonance Spectroscopy, Eds. L. M. JACKMAN and F. A. COTtON, p. 203, Academic Press, New York. (1975).

193. J. Hauer, G. Völkel and H.-D. Lüdemann, Chem. Phys. Lett. 78, 85 (1980).

194. J. Hauer, G. Völkel and H.-D. Lüdemann, J. Chem. Res. (S) 16; (M) 0426 (1980).

195. H.-D. LódemanN, R. RauchsChWaLeE and E. W. LanG, Angew. Chem. Int. Ed. 16, 331 (1977); 89, 340 (1977).

196. R. Rauchschwalbe, G. Völokel, E. W. Lang and H.-D. LüdemanN, J. Chem. Res. (S) 448 (1978); (M) 5325 (1978).

197. J. Hauer, E. Treml, H.-D. Lúdemann and A. MannsChreck, J. Chem. Res. (S) 14 (1982); (M) 0253 (1982).

198. J. Hauer, E. Treml and H.-D. Lüdemann, J. Chem. Res. (S) 40 (1982); (M) 0501 (1982).

199. J. Hauer, E. Treml and H.-D. Lüdemann, J. Chem. Res. (S) 42 (1982); (M) 0516 (1982).

200. J. HAUER, H.-D. LüDEMANN and R. JAENICKE, Z. Naturforsch. 37c, 51 (1982).

201. J. Hauer, H.-D. LüdemanN and J. W. C. Verhoeven, $Z$. Naturforsch. $36 c$, 366 (1981).

202. H.-D. LÜDEMANN, E. W. LANG and E. WeSTHOF, FEBS Lett. 80, 107 (1977).

203. G. Klimke, J. Hauer, H.-D. Lüdemann and W. Pfleiderer, J. Chem. Res. 80 (1981).

204. G. Völkel, E. W. Lang and H.-D. Lódemann, Ber, Bunsenges. Phys. Chem. 83, 722 (1979).

205. T. Drakenaerg, K.-I. Dahlqvist and S. Forsen, J. Phys. Chem. 76, 2178 (1972).

206. A. G. Whittaker and S. Siegel, J. Chem. Phys. 42, 3320 (1965).

207. L. W. Rewes, R. C. Shaddick and K. N. Shaw, Can. J. Chem. 49, 3683 (1971).

208. L. M. Jackman, T. E. Kavanaugh and R. C. Haddon, Org. Magn. Reson. 1, 109 (1969).

209. K. Spaargaren, P. K. Korver, P. J. van der HaAK and T. J. de Boer, Org. Magn. Reson. 3, 615 (1971).

210. J. Jonas and X. Peng, Ber Bunsenges. Phys. Chem. 95, 243 (1991).

211. D. L. Hasha, T. EguCHI and J. Jonas, J. Chem. Phys. 75, 1571 (1981).

212. D. L. Hasha, T. EguCHI and J. Jonas, J. Am. Chem. Soc. 104, 2290 (1982).

213. J. Ashcroft, M. Besnard, V. Aquada and J. Jonas, Chem. Phys. Lett. 110, 420 (1984).

214. J. AshCroft, Ch.-L. XIE and J. Jonas, J. Chem. Phys. 90, 5386 (1989).

215. D. M. Campbell, M. Mackowiak and J. Jonas, J. Chem. Phys. 96, 2717 (1992).

216. G. WAGNER, FEBS Lett. 112, 280 (1980). 
217. P. W. E. Peereboom, H. Luiggis and K. O. Prins, Physica A 156, 260 (1989).

218. K. R. HARris, Physica A 94, 448 (1978).

219. K. R. HARRIS, L. A. WOOLF, J. Chem. Soc. Faraday Trans. I, 76, 377 (1980).

220. A. Greiner-Schmid, S. Wappmann, M. Has and H.-D. Lödemann, J. Chem. Phys. 94, 5643 (1991).

221. M. HAS and H.-D. LüdemanN, J. Mol. Liquids 46, 7 (1990).

222. R. Lustig and W. A. Steele, Mol. Phys. 65, 475 (1988).

223. S. Gupta, J. Yang and N. D. Kestner, J. Chem. Phys. 89, 3733 (1988).

224. S. Toxvaerd, J. Chem. Phys. 91, 3716 (1989).

225. F. X. Prielmeier and H.-D. Lödemann, Mol. Phys. 58, 593 (1986).

226. M. A. MC CoOL and L. A. WoOLF, J. Chem. Soc. Faraday Trans. I, 66, 1471 (1972).

227. E. W. Lang, F. X. Prielmeier, H. Radkowitsch and H.-D. Lüdemann, Ber. Bunsenges. Phys. Chem. 85, 1017 (1987).

228. E. W. Lang, F. X. Prielmeier, H. Radkowitsch and H.-D. Lüdemann, Ber. Bunsenges. Phys. Chem. 85, $1025(1987)$.

229. T. Vardag and H.-D. Lúdemann, Chem. Phys. 128, 527 (1988).

230. M. HAS and H.-D. LÜDEMANN, Z. Naturforsch. 44a, 1210 (1989).

231. K. R. Harris, H. N. Lam, E. Raedt, A. J. Easteal, W. E. Price and L. A. Woolf, Mol. Phys. 71, 1205 (1990).

232. J. De ZwaAn, J. Jonas, J. Chem. Phys. 62, 4036 (1975).

233. J. Jonas, D. Hasha and S. G. Huang, J. Phys. Chem. 84, 109 (1980).

234. D. L. Hasha, PhD Thesis, University of Illinois, Urbana (1981).

235. T. VardaG, F. Bachl, S. WaPpManN and H.-D. Lüdemann, Ber. Bunsenges. Phys. Chem. 94, 336 (1990).

236. F. BACHL and H.-D. LÜDEMANN, Z. Naturforsch. 41a, 963 (1986).

237. T. Vardag, S. Wappmann and H.-D. Lüdemann, Erdöl Kohle, Erdgas Petrochemie 44, 342 (1991).

238. J. H. Dymond and K. R. Harris, Mol. Phys. 75, 461 (1992).

239. J. Jonas, D. L. Hasha and S. G. HuanG, J. Chem. Phys. 71, 3996 (1979).

240. M. Wozny, F. X. Prielmeier and H.-D. Lüdemann, Z. Naturforsch. 39a, 800 (1984).

241. F. Bachl, T. VARDaG, S. Wappmann and H.-D. LODEmanN, J. Mol. Liquids, 54, 193 (1992).

242. B. Arends, K. O. Prins and N. J. Trappeniers, Magn. Reson. Relat. Phenom. Proc. Congr. AMPERE 20th., Eds. E. Kundla, E. LippMaA and T. SAluvere, p. 463, Springer, Berlin (1979).

243. B. Arends, K. O. Prins and N. J. Trappeniers, Physica A 107A, 307 (1981).

244. E. S. Baker, D. R. Brown and J. Jonas, J. Phys. Chem. 88, 5425 (1984).

245. P. G. de Gennes, J. Chem. Phys. 55, 572 (1971).

246. P. E. Rouse, J. Chem. Phys. 21, 1272 (1953).

247. S. F. Edward, Proc. Phys. Soc. 92, 9 (1967).

248. S. F. Edwards, Polymer 9, 140 (1977).

249. M. H. COHEN and G. S. Grest, Phys. Rev. B20, 1077 (1979).

250. J. D. Ferry, Viscoelastic Properties of Polymers 3rd edn, John Wiley, New York (1980).

251. N. A. Walker, D. M. Lamb, S. T. Adamy, J. Jonas and M. P. Dare-EdWards, J. Phys. Chem. 92,3675 (1988).

252. H. G. Parkhurst and J. Jonas, J. Chem. Phys. 63, 2698 (1975).

253. M. Fury, G. Munie and J. Jonas, J. Chem. Phys. 70, 1260 (1979).

254. R. J. Finney, M. Fury and J. Jonas, J. Chem. Phys. 66, 760 (1977).

255. R. Malhotra, W. E. Price, L. A. Woolf and A. J. Easteal, Int. J. Thermophys. 11, 835 (1990).

256. J. De ZwaAn and J. Jonas, J. Chem. Phys. 63, 4606 (1975).

257. A. Greiner-SChmid, M. HAS and H.-D. LübemanN, Z. Naturforsch. 45a, 1281 (1990).

258. K. Krynicki, A.-L. Meragi and J. G. Powles, Ber. Bunsenges. Phys. Chem. 85, 1153 (1981).

259. K. Krynicki, S. N. Changdar and J. G. Powles, Mol. Phys. 39, 773 (1980).

260. V. V. KiselNik, N. G. MalyUK, A. N. TORYANIK and V. M. TORYANIK, Zh. Strukt. Khim. (English Translation), 14, 963 (1973).

261. L. A. WOOLF, J. Chem. Phys. 61, 1600 (1974).

262. C. A. Angell, E. D. Finch, L. A. Woolf and P. BaCh, J. Chem. Phys. 65, 3063 (1976).

263. K. Krynicki, C. D. Green and D. W. Sawyer, Faraday Disc. Chem. Soc. 66, 199 (1980).

264. L. A. Woolf and K. R. Harris, High Pressure Sci. Technol., Vol. 2, Eds. B. Vodar and Ph, Marteau, p. 702, Pergamon Press, Oxford (1980).

265. W. J. Lamb, G. A. Hoffmann and J. Jonas, J. Chem. Phys. 74, 6875 (1981).

266. F. X. Prielmeier, E. W. Lang., R. J. Speedy and H.-D. Lüdemann, Phys. Rev. Lett. 59, 1128 (1987).

267. F. X. Prielmeier, E. W. Lang, R. J. Speedy and H.-D. Lúdemann, Ber. Bunsenges. Phys. Chem. 92, 1111 (1988).

268. D. J. Wilbur, T. De Fries and J. Jonas, J. Chem. Phys. 65, 1783 (1976).

269. T. De Fries and J. Jonas, J. Chem. Phys. 66, 5393 (1977).

270. R. L. Hurle, A. J. Easteal and L. A. Wool.F, J. Chem. Soc. Faraday Trans. I, 81, 769 (1985).

271. N. Karger, T. Vardag and H.-D. Lüdemann, J. Chem. Phys. 93, 3437 (1990).

272. J. Jonas and J. A. AKal, J. Chem. Phys. 66, 4946 (1977).

273. S. MeCKL and M. D. ZeidLer, Mol. Phys. 63, 85 (1988).

274. J. A. AKAl and J. JONAS, J. Solut. Chem. 5, 563 (1976). 
275. E. S. Baker and J. Jonas, J. Phys. Chem. 89, 1730 (1985).

276. M. WozNYl, PhD Thesis, Universität Regensburg (1985).

277. M. HAs, PhD Thesis, Universität Regensburg (1991).

278. M. Bruesewitz, A. Weiss, Ber Bunsenges. Phys. Chem. 94, 386 (1990).

279. U. El-Hanany, G. F. Brennert and W. W. Warren, Phys. Rev. Lett. $\mathbf{5 0 ,} 540$ (1983).

280. W. W. Warren, U. El-Hanany and G. F. Brennert, J. Non-Cryst. Solids 61, 23 (1984).

281. J. Korringa, Physica 16, 601 (1950).

282. T. MoriYa, J. Phys. Soc. Jpn. 18, 516 (1963).

283. W. W. Warren and R. Dupree, Phys. Rev. B 22, 2257 (1980).

284. A. Koma and S. Tanaka, Solid State Commun. 10, 823 (1972).

285. R. L. Armstrong and P. A. SPEIGHT, J. Magn. Reson. 2, 141 (1970).

286. M. BloOM, M. LiPsicas and B. H. Muller, Can. J. Phys. 39, 1093 (1961).

287. C. S. Johnson and J. S. Waugh, J. Chem. Phys. 35, 2020 (1961).

288. C. S. Johnson and J. S. Waugh, J. Chem. Phys. 36, 2266 (1962).

289. M. BloOM, F. Bridges and W. N. Hardy, Can. J. Phys. 45, 3533 (1967).

290. N. J. Trappeniers, C. J. Gerritsma and P. H. Oosting, Physica 31, 202 (1965).

291. A. A. Maryott, M. S. Malmberg and K. T. Gillen, Chem. Phys. Lett. 25, 169 (1974).

292. M. Bloom and I. OpPenheim, Can. J. Phys. 41, 1580 (1963).

293. P. H. Oosting and N. J. Trappeniers, Physica 51, 395 (1971).

294. W. N. Hardy, Can. J. Phys. 44, 265 (1966).

295. M. Bloom and R. Dorothy, Can. J. Phys. 45, 3411 (1967).

296. P. A. Beckmann, M. Bloom and E. Burnell, Can. J. Phys. 50, 251 (1972).

297. G. DE WIT and M. BLoom, Can. J. Phys. 43, 986 (1965).

298. C. J. Gerritsma, P. H. Oosting and N. J. Trappeniers, Physica 51, 381 (1971).

299. C. J. Gerritsma and N. J. Trappeniers, Physica 51, 365 (1971).

300. P. H. Oosting and N. J. Trappeniers, Physica 51, 418 (1971).

301. M. Bloom and H. S. SAndhu, Can. J. Phys. 40, 289 (1962).

302. J. H. Rugheimer and P. S. Hubbard, J. Chem. Phys. 39, 552 (1963).

303. P. S. Hubbard, Phys. Rev. A 6, 2421 (1972).

304. H. S. Gutowsky, I. J. Lawrenson and K. Shimomura, Phys. Rev. Lett. 6, 349 (1961).

305. R. Y. Dong and M. Bloom, Phys. Rev. Lett. 20, 981 (1968).

306. R. Y. Dong and M. Bloom, Can. J. Phys. 48, 793 (1970).

307. R. L. Armstrong and J. A. Courtney, Can. J. Phys. 50, 1262 (1972).

308. S. PAusak and J. S. WAUGH, J. Chem. Phys. 61, 2165 (1974).

309. C. S. Johnson, J. S. Waugh and J. N. Pinkerton, J. Chem. Phys. 35, 1128 (1961).

310. J. H. Chaffin and P. S. Hubbard, J. Chem. Phys. 46, 1511 (1967).

311. J. W. HarReLl, J. Magn. Reson. 23, 335 (1976).

312. A. Walton, J. C. Pratt, E. C. Reynhardt and H. E. Petch, J. Chem. Phys. 77, 2344 (1982).

313. E. W. Lang, F. X. Prielmeier, H. Radkowitsch and H.-D. LÜdemann, Ber. Bunsenges. Phys. Chem. 91, 1017 (1987).

314. E. W. Lang, F. X. Prielmeier, H. Radkowitsch and H.-D. Lúdemann, Ber. Bunsenges, Phys. Chem. 91, 1025 (1987).

315. E. A. C. Lucken, Nuclear Quadrupole Coupling Constants, Academic Press, London (1969).

316. A. Gerschel, I. Dimicoli, J. Jaffre and A. Rion, Mol. Phys. 32, 679 (1976).

317. W. H. FurRY, Phys. Rev. 107, 7 (1957).

318. R. E. D. MC ClunG, Adv. Mol. Relax. Interact. Proc. 10, 83 (1977).

319. R. G. Gordon, J. Chem. Phys. 44, 1830 (1966).

320. R. E. D. MC Clung, J. Chem. Phys. 73, 2435 (1980).

321. H. J. Böhm, C. Meissner and R. Ahlrichs, Mol. Phys. 53, 651 (1984).

322. A. Gerschel, C. Brot, I. Dimicolı and A. Rion, Mol. Phys. 33, 527 (1977).

323. R. M. Lynden-Bell, Molecular Liquids. Eds. A. J. Barnes, W. J. Orville-Thomas and J. Yarwood, Reidel, Dordrecht (1984).

324. R. E. D. MC Clung, J. Chem. Phys. 51, 3842 (1969).

325. R. E. D. MC Clung, J. Chem. Phys. 57, 5478 (1972).

326. J.-CL. LeICKNAM, Phys. Rev. A 222286 (1980).

327. J. Lascomb, M. Besnard and P. Maraval, Chem. Phys. 72, 177 (1982).

328. T. E. Bull and W. Egan, J. Chem. Phys. 81, 3181 (1984).

329. P. S. Hubbard, Phys. Rev. A 24, 645 (1981).

330. G. Levi, J. P. Marsault, F. Marsault-Herail and R. E. D. Mc Clung, J. Chem. Phys. 73, 2443 (1980).

331. R. E. D. MC Clung, J. Chem. Phys. 75, 5503 (1981).

332. D. H. LeE and R. E. D. MC Clung, Chem. Phys. 112, 23 (1987).

333. D. H. LeE and R. E. D. MC CLunG, J. Magn. Reson. 73, 34 (1987).

334. A. Gerschel, I. Dimicoli, J. Jofrre and A. Rion, Mol. Phys. 32, 679 (1976).

335. A. Gerschel, J. Mol. Liquids 25, 51 (1983). 
336. R. L. Armstrong and E. Tward, J. Chem. Phys. 48, 332 (1968).

337. J. K. Tison and E. R. Hunt, J. Chem. Phys. 54, 1526 (1971).

338. S. Mohanty and H. J. Bernstein, J. Chem. Phys. 54, 3730 (1971).

339. J. A. Courtney and R. L. Armstrong, Can. J. Phys. 50, 1252 (1972).

340. J. H. Campbell, S. J. Seymour and J. Jonas, J. Chem. Phys. 59, 4151 (1973).

341. R. J. Finney, M. Wolfe and J. JonaS, J. Chem. Phys. 67, 4004 (1977).

342. M. Wolfe, E. Arndt and J. Jonss, J. Chem. Phys. 67, 4012 (1977).

343. R. G. Gordon, R. L. Armstrong and E. Tward, J. Chem. Phys. 48, 2655 (1968).

344. F. Khoury and R. Kobayashi, J. Chem. Phys. 55, 2439 (1971).

345. J. H. Camprell, J. F. Fisher and J. Jonas, J. Chem. Phys. 61, 346 (1974).

346. W. T. HUNTRESS, Adv. Magn. Reson. 4, 1 (1970).

347. H. W. SPiess, D. SChWeitzer and V. HaEBerleiN, J. Magn. Reson. 9, 444 (1973).

348. D. E. WOESSNER and B. S. SNOWden, 8th Colloquium of NMR Spectroscopy, Aachen (1971).

349. D. L. van der Hart, J. Chem. Phys. 60, 1858 (1974).

350. A. W. Nolle and P. P. Mahendroo, J. Chem. Phys. 33, $863(1960)$.

351. J. H. CampBell and J. Jonas, Chem. Phys. Lett. 18, 441 (1973).

352. J. L. Ragle and K. L. Sherk, J. Chem. Phys. 50,3553 (1969).

353. S. Glasstone, K. J. Laidler and H. Eyring, The Theory of Rate Processes, McGraw-Hill, New York (1941).

354. T. E. Bull and J. Jonas J. Chem. Phys. 52, 2779 (1970).

355. R. A. Assink, J. De ZwaAn and J. Jonas, J. Chem. Phys. 56, 4975 (1972).

356. J. Batchinski, Z. Phys. Chem. 84, 643 (1913).

357. R. E. D. MC Clung and D. Kivelson, J. Chem. Phys. 49, 3380 (1968).

358. M. Fury and J. Jonas, J. Chem. Phys. 65, 2206 (1976).

359. M. H. Cohen and D. Turnbull, J. Chem. Phys. 31, 1164 (1959).

360. W. M. Madigosky and T. A. Litovitz, J. Chem. Phys. 34, 489 (1961).

361. J. De ZwaAn and J. Jonas, J. Chem. Phys. 62, 4036 (1975).

362. K. T. Gillen, D. C. Douglass, M. S. Malmberg and A. A. Maryott, J. Chem. Phys. 57, 5170 (1972).

363. R. E. D. MC Clung, Chem. Phys. Lett. 19, 304 (1973).

364. J. De ZwaAn and J. Jonas, J. Chem. Phys. 63, 4606 (1975).

365. S. Sunder and R. E. D. MC Clung, Can. J. Phys. 52, 1209 (1977).

366. J. H. Dymond, J. 'Chem. Phys. 60, 969 (1974).

367. Z. M. El Saffar and P. Schultz, J. Chem. Phys. 56, 2524 (1971).

368. J. H. Loehlin, P. G. Mennitt and J. S. Waugh, J. Chem. Phys. 44, 3912 (1966).

369. D. C. LOOK, I. J. Lowe and J. A. NORThBY, J. Chem. Phys. 44, 3441 (1966).

370. J. W. Hennel, A. Jasinski, S. Sagnowski and T. Waluga, Acta Phys. Polon. 28, 673 (1965).

371. D. E. O'reilly and J. H. Eraker, J. Chem. Phys. 52, 2407 (1970).

372. R. Topol, K. Krynicki and J. G. Powles, Mol. Phys. 37, 1655 (1979).

373. R. TOPOL, Mol. Phys. 40, 161 (1980).

374. J. C. Hindman, A. Svirmickas and W. B. Dixon, J. Chem. Phys. 47, 4658 (1967).

375. H. Rzany and J. SCIesinski, Physica 29, 488 (1963).

376. F. C. De Lucia and J. W. Cederberg, J. Mol. Spectrosc. 40, 52 (1971).

377. R. L. Cook, F. C. De Lucia and P. Helminger, J. Mol. Struct. 28, 237 (1975).

378. J. G. Powles and G. Rickayzen, Mol. Phys. 33, 1207 (1977).

379. G. B. Benedek and E. M. Purcell, J. Chem. Phys. 22, 2003 (1954).

380. T. E. Bull and J. Jonas, J. Chem. Phys. 52, 2779 (1970).

381. M. D. ZeidLer, Ber. Bunsenges. Phys. Chem. 69, 659 (1965).

382. J. G. Powles and M. C. Gough, Mol. Phys. 16, 349 (1969).

383. C. G. WADE and J. S. Whugh, J. Chem. Phys. 43, 3555 (1965).

384. T. E. Bull and J. Jonas, J. Chem. Phys. 53, 3315 (1970).

385. A. Allerhand, J. Chem. Phys. 52, 3596 (1970).

386. J. LeEs, B. H. MülleR and J. D. NoBle, J. Chem. Phys. 34, 341 (1961).

387. S. G. G. KaShaer, B. Le and M. Z. Zinyakov, Dokl. Akad. Nauk. SSSR B157, 1438 (1964).

388. A. K. DoolitTLE, I. Simon and R. A. CoRnish, A.I.Ch.E.Jl. 6, 150 (1960).

389. A. A. Brooks, B. D. Boss, E. O. Stejskal and V. W. Weiss, J. Chem. Phys. 48, 3826 (1968).

390. D. W. MC Call, D. C. Douglass and E. W. Anderson, J. Chem. Phys. 31, 1555 (1959).

391. P. W. Bridgman, Proc. Am. Acad. Arts. Sci. 61, 57 (1926).

392. D. E. Woessner and B. S. Snowden, J. Chem. Phys. 52, 1621 (1970).

393. E. R. ANDrew and R. G. EAdes, Proc. Roy. Soc. A 218, 537 (1953).

394. T. E. Bull and J. Jonas, J. Chem. Phys. 52, 4553 (1970).

395. J. G. Powles, M. Rhodes and J. H. Strange, Mol. Phys. 11, 515 (1966).

396. D. J. Wilbur and J. Jonas, J. Chem. Phys. 55, 5840 (1971).

397. D. J. WILBur and J. Jonas, J. Magn. Reson. 10, 279 (1973).

398. H. J. Parkhurst, Y. LeE and J. Jonas, J. Chem. Phys. 55, 1368 (1971).

399. A. Bondi, J. Phys. Chem. 68, 441 (1964). 
400. R. A. Assink, J. De Zwann and J. Jonas, J. Chem. Phys. 56, 4975 (1972).

401. D. Kivelson, M. G. Kivelson and I. Oppenheim J. Chem. Phys. 52, 1810 (1969).

402. J. De ZwaAn and J. Jonas, J. Phys. Chem. 77, 1768 (1973).

403. D. J. Wilbur and J. Jonas, J. Chem. Phys. 62, 2800 (1975).

404. D. Wallach, J. Phys. Chem. 73, 307 (1969).

405. A. Gierer and K. WirTZ, Z. Naturforsch. A 8, 532 (1953).

406. H. W. SPIESS, D. SChWeitzer and U. HAeberLen, J. Magn. Reson. 9, 444 (1973).

407. R. G. PARKeR and J. JonAs, J. Magn. Reson. 6, 106 (1972).

408. D. E. O'reilly and E. M. Peterson, J. Chem. Phys. 56, 2262 (1972).

409. R. G. Barnes and J. W. Bloom, J. Chem. Phys. 57, 3082 (1972).

410. J. C. Rowell, W. D. Phillips, L. R. Melay and M. Panar, J. Chem. Phys. 43, 3442 (1965).

411. R. G. Gordon, R. L. Armstrong and E. Tward, J. Chem. Phys. 48, 2655 (1968).

412. R. A. Assink and J. Jonas, J. Chem. Phys. 57, 3329 (1972).

413. J. De Zwan,, R. J. Finney and J. Jonas, J. Chem. Phys. 60, 3223 (1974).

414. D. K. Green and J. G. Powles, Proc. Phys. Soc. 85, 87 (1965).

415. S. I. Chan and A. S. Dubin, J. Chem. Phys. 46, 1745 (1967).

416. E. Wilhelm, J. Chem. Phys. 58, 3558 (1973).

417. I. ARTAKI and J. Jonas, Mol. Phys. 55, 867 (1985).

418. E. ARndt and J. Jonas, J. Phys. Chem. 85, 463 (1981).

419. A. J. Barlow, J. Lamb and A. J. Matheson, Proc. R. Soc. A 292, 322 (1966).

420. G. JoharI and M. GoldsteIn, J. Chem. Phys. 53, 2372 (1970).

421. D. Davies and A. Matheson, J. Chem. Phys. 45, 1000 (1966).

422. I. ArtakI and J. Jonas, J. Chem. Phys. 82, 3360 (1985).

423. J. Jonas, S. T. Adamy, P. J. Grandinetti, Y. Masuda, S. J. Morris and Y. Li, J. Phys. Chem. 94,1157 (1990).

424. S. T. Adamy, D. J. Grandinetti, Y. Masuda, D. CAmpbell and J. Jonas, J. Chem. Phys. 94, 3568 (1991).

425. N. A. Walker, D. M. Lamb, J. Jonas and M. P. Dare-Edwards, J. Magn. Reson. 74, 580 (1987).

426. N. A. Walker, D. H. LamB, S. T. Adamy and J. Jonas, J. Phys. Chem. 92,3675 (1988).

427. R. B. Fiorito and R. Meister, J. Chem. Phys. 56, 4605 (1972).

428. J. P. Kintzinger and M. D. Zeidler, Ber. Bunsenges. Phys. Chem. 77, 98 (1973).

429. R. KuBo and K. Tomita, J. Phys. Soc. Jpn. 9, 888 (1954).

430. F. Noack and G. Preissing, Z. Naturforsch. 24A, 143 (1969).

431. K. Kuszczynski, J. Kall and J. G. Powles, Proc. Phys. Soc. 75, 243 (1960).

432. T. ConNOR, Trans. Faraday Soc. 60, 1574 (1964).

433. F. Nonck and G. Preissing, Verhandl. Deutsch. Phys. Ges. VI 5, 347 (1970).

434. E. O. Stejskal and J. TANner, J. Chem. Phys. 42, 288 (1965).

435. M. Wolfe and J. Jonas, J. Chem. Phys. 71, 3252 (1979).

436. L. J. Burnett and S. B. W. Roeder, J. Chem. Phys. 60, 2420 (1974).

437. P. Drake and R. Meister, J. Chem. Phys. 54, 3046 (1971).

438. J. T. Hynes, R. KAPral and W. Weinberg, J. Chem. Phys. 69, 2725 (1978).

439. W. G. S. SCAIFE, J. Phys. D 9, 1489 (1976).

440. C. Demoulin, C. J. Montrose and N. Ostrowsky, Phys. Rev. A 9, 1740 (1974).

441. A. H. Narten, J. Chem. Phys. 66, 3117 (1977).

442. J. L. Carolan and T. A. SCOTT, J. Magn. Reson. 2, 243 (1970).

443. W. M. Litchman and M. Alei, J. Chem. Phys. 56, 5818 (1972).

444. W. G. SCHNEIDER, Symposium on Hydrogen Bonding, Ljubliana (1957).

445. J. G. Powles and M. RHODES, Mol. Phys. 12, 399 (1967).

446. D. W. G. SMITH and J. G. Powles, Mol. Phys. 10, 451 (1966).

447. J. G. Powles, H. Rhodes and J. H. STRANGe, Mol. Phys. 11, 515 (1966).

448. P. W. Atkins, A. Loewenstein and Y. Margalit, Mol. Phys. 17, 329 (1969).

449. J. Hauer, E. W. Lang and H.-D. LÚdemanN, Ber. Bunsenges. Phys. Chem. 83, 1262 (1979).

450. J. G. Powles and G. Rickayzen, Mol. Phys. 33, 1207 (1977).

451. R. M. Garvey, F. C. De Lucia and J. W. Cederberg, Mol. Phys. 31, 265 (1976).

452. E. W. LANG and H.-D. LOdemann, J. Chem. Phys. 67, 718 (1977).

453. F. Franks, Ed, Water - A Comprehensive Treatise, Vols 1-7, Plenum Press, New York (1972-1982).

454. E. W. Lang and H.-D. LUdemann, NMR-Basic Principles and Progress. Vol. 24, Ed. J. Jonas, p. 129, Springer, Berlin (1990).

455. H. G. HerTZ and C. RädLE, Z. Phys. Chem. N. F. 68, 324 (1969).

456. Y. LEE and J. Jonas, J. Chem. Phys. 57, 4233 (1972).

457. J. Jonas, T. DE Fries and D. J. WiLBUR, J. Chem. Phys. 65, 582 (1976).

458. T. De Fries and J. Jonas, J. Chem. Phys. 66, 5393 (1977).

459. Y. LEE and J. JONAS, J. Magn. Reson. 5, 267 (1971).

460. J. H. Wang, C. V. Robinson and I. S. Edelman, J. Am. Chem. Soc. 75, 466 (1953).

461. H. R. Pruppacher, J. Chem. Phys. 56, 101 (1972).

462. R. BlinC, Adv. Magn. Reson. 3, 157 (1968). 
463. J. C. Hindman, A. Svirmickas and M. Wood, J. Chem. Phys. 59, 1517 (1973).

464. K. KRYNicki, Physica 32, 167 (1966).

465. V. V. Kiselnik, N. G. Malyuk and A. N. Toryanik, Zh. Strukt. Khim. 15, 697 (1974).

466. F. H. Stillinger and A. Rahman, J. Chem. Phys. 61, 4973 (1974).

467. A. Y. Wu, E. WhalleY and G. Dolling, Mol. Phys. 47, 603 (1982).

468. T. De Fries and J. Jonas, J. Chem. Phys. 66, 896 (1977).

469. D. H. Rassmussen, A. P. MC Kenzie, Water Structure and the Water-Polymer Interface, Ed. H. H. Jellinek, Plenum Press, New York (1972).

470. H. KanNo, R. J. Speedy and C. A. ANGell, Science, 189, 880 (1975).

471. J. C. Hindman, A. Svirmickas and M. WoOd, J. Phys. Chem. 74, 1266 (1970).

472. J. C. Hindman, A. J. Zielen, A. Svirmickas and M. Wood, J. Chem. Phys. 54, 621 (1971).

473. J. Hindman, J. Chem. Phys. 60, 4488 (1974).

474. E. W. LANG, PhD Thesis, Universität Regensburg (1980).

475. E. W. Lang and H.-D. Lüdemann, Ber. Bunsenges. Phys. Chem. 84, 462 (1980).

476. E. W. Lang and H.-D. Lüdemann, Ber. Bunsenges, Phys. Chem. 85, 603 (1981).

477. E. W. Lang and H.-D. Lüdemann, Ber. Bunsenges. Phys. Chem. 85, 1016 (1981).

478. E. W. Lang, L. Piculell and H.-D. Lúdemann, J. Chem. Phys. 81, 3820 (1984).

479. E. W. Lang, D. Girlich, H.-D. Lüdemann, L. Piculell and D. Múller, J. Chem. Phys. 93,4796 (1990).

480. M. SCEATS and S. A. RICE, Water - A Comprehensive Treatise, Vol. 7, Ed. F. Franks, p. 83, Plenum Press, New York (1982).

481. D. T. Edmonds, S. D. Goren, A. L. Mackay and A. A. White, J. Magn. Reson. 23, 505 (1976).

482. A. Weiss and N. WeIDEN, Adv. Nucl. Quadr. Resan, 4, 149 (1980).

483. B. Berglund, J. Lindiren and J. Tegenfeldt, J. Mol. Struct. 43, 179 (1978).

484. R. Chidambaram and S. K. SikKa, Chem. Phys. Lett. 2, 162 (1968).

485. C. A. ANGell, Water - A Comprehensive Treatise, Vol. 7, Ed. F. Franks, p. 1, Plenum Press, New York (1982).

486. R. J. SpeEdy, J. Phys. Chem. 86, 982 (1982).

487. R. J. SPEedy and C. A. ANgell, J. Chem. Phys. 65, 851 (1976).

488. G. W. Robinson, J. Lee, K. G. Casey and D. Statman, Chem. Phys, Lett. 123, 483 (1986).

489. G. W. Robinson, J, Lee and M. -P. BASSEZ, J. Chem. Soc. Faraday Trans. II 82, 2351 (1986).

490. G. W. Robinson, J. Lee and M. -P. Bassez, Chem. Phys. Lett. 137, 376 (1987).

491. M. -P. Bassez, J. Lee and G. W. Robinson, J. Phys. Chem. 91, 5818 (1987).

492. D. Lankhorst, J. Schriever and J. C. Leyte, Ber. Bunsenges. Phys. Chem. 86, 215 (1982).

493. J. G. Powles, Mol. Phys. 42, 757 (1981).

494. J. Jonas, T. De Fries and W. J. Lamb, J. Chem. Phys. 68, 2988 (1978).

495. W. J. LAMB and J. Jonas, J. Chem. Phys. 74, 913 (1981).

496. K. Lalita and M. Bloom, Chem. Phys. Lett. 8, 285 (1971).

497. H. C. Anderson, D. Chandler and J. D. Weeks, Adv. Chem. Phys. 34, 105 (1976).

498. S. Meiвoom, Z. Luz and D. Gill, J. Chem. Phys. 27, 1411 (1957),

499. S. Meiboom, J. Chem. Phys. 34, 375 (1961).

500. Z. LuZ and S. Meiвoom, J. Am. Chem. Soc. 86, 4766 (1964).

501. R. R. KNisPel and M. M. PINTAR, Chem. Phys. Lett. 32, 238 (1975).

502. V. Graf, F. Noack and G. J. Bene, J. Chem. Phys. 72, 861 (1980).

503. S. W. Rabideau and H. G. HeCht, $J$. Chem. Phys. 47, 544 (1967).

504. H. G. Hertz and R. Klute, Z. Phys. Chem. N.F. 64, 101 (1970).

505. D. L. Turner, Mol. Phys. 40, 949 (1980).

506. B. Halle and G. KARLSTRÖM, J. Chem. Soc. Faraday Trans. II. 79, 1031 (1983).

507. B. HALLE and G. KARL.STRÖM, J. Chem. Soc. Faraday Trans. II. 79, 1047 (1983).

508. W. J. Lamb, D. R. Brown and J. Jonas, J. Phys. Chem. 85, 3883 (1981).

509. M. D. Zeidler, Water - A Comprehensive Treatise, Vol. 2, Ed. F. Franks, p. 529, Plenum Press, New York (1973).

510. K. Hallenga, J. R. Grigera and H. J. C. Berendsen, J. Phys. Chem. 84, 2381 (1980).

511. K. J. PACKer, Phil. Trans. R. Soc. London B 278, 59 (1977).

512. R. Mathur-De VRe, Prog. Biophys. Mol. Biol. 35, 103 (1979).

513. N. S. IsaAcs, Liquid Phase High Pressure Chemistry, Wiley, Chichester (1981).

514. R. E. Marquis and P. Matsumara, Microbial Life in Extreme Environments, Ed. D. J. Kushnev, p. 105, Academic Press, London (1978).

515. Y. Lee and J. Jonas, J. Chem. Phys. 59, 4845 (1973).

516. H. S. Frank and M. W. Evans, J. Chem. Phys. 13, 507 (1945).

517. C. D. Leake, Biological Actions of Dimethyl Sulfoxide, Ann. N. Y. Acad. Sci., New York (1967).

518. G. J. Safford, P. C. Schaffer, P. S. Leuny, G. F. Doebrler, G. W. Brady and F. F. X. Lyden, J. Chem. Phys. 50, 2140 (1969).

519. E. S. BaKer and J. Jonas, J. Phys. Chem. 89, 1730 (1985).

520. L. A. Wool.F, Pure Appl. Chem. 57, 1083 (1985). 
521. A. J. Easteal and L. A. Woolf, J. Phys. Chem. 89, 1066 (1985).

522, B. L. Halfpap and C. M. Sorensen, J. Chem. Phys. 77, 466 (1982).

523. C. M. Sorensen, J. Chem. Phys. 79, 1455 (1983).

524. C. A. Angell, Ann. Rev. Phys. Chem. 34, 593 (1983).

525. E. V. Goldammer and H. G. Hertz, J. Phys. Chem. 74, 3734 (1970).

526. G. W. Enliss and C. M. Sorensen, J. Chem. Phys. 80, 4767 (1984).

527. M. WozNYJ, PhD Thesis, Universität Regensburg (1985).

528. J. E. EnDerby, The Physics and Chemistry of Aqueous Ionic Solutions, NATO ASI Ser. C 205, Eds. M. C. BeLLISSENT-Funel and G. W. NeILsON, p.129, (1987).

529. K. Heinzinger, Physica 131B, 196 (1985).

530. M. Holz, Prog. NMR Spectrosc. 18, 327 (1986).

531. H. G. HerTz, Water - A Comprehensive Treatise, Vol. 3, Ed. F. FPadks, p. 301, Plenum Press, New York (1973).

532. H. G. Hertz, Pure Appl. Chem. 54, 2297 (1982).

533. R. G. Bryant, Ann. Rev. Phys. Chem. 29, 167 (1978).

534. H. Langer and H. G. Hertz, Ber. Bunsenges. Phys. Chem. 81, 478 (1977).

535. A. Geiger and H. G. HertZ, J. Sol. Chem. 5, 365 (1976).

536. H. L. Friedman, Chem. Scr. 25, 42 (1985).

537. J. A. AKal and J. Jonas, J. Sol. Chem. 5, 563 (1976).

538. P. M. Gammell and R. Meister, J. Chem. Phys. 64, 4287 (1976).

539. B. M. Braun and H. Weingärtner, J. Phys. Chem. 92, 1342 (1988).

540. K. TanaKa and M. Nomura, J. Chem. Soc. Faraday Trans. I. 83, 1779 (1987).

54I. D. R. MaC Farlane, J. Scheirer and S. J. Smedley, J. Phys. Chem. 90, 2168 (1986).

542. Y. LeE and J. JoNAS, J. Magn. Reson. 5, 267 (1971).

543. Y. K. LeE, J. H. Campbell and J. Jonas, J. Chem. Phys. 60, 3537 (1974).

544. J. Jonas, D. Brown and J. J. Fripiat, J. Colloid Interface Sci. 89, 374 (1982).

545. E. W. Lang, W. Fink and H.-D. Lüdemann, J. Phys. C, 45, C7-137 (1984).

546. E. W. Lang and H.-D. Lüdemann, Ber. Bunsenges. Phys. Chem. 89, 508 (1985).

547. E. W. Lang and F. X. Prielmeier, Ber. Bunsenges. Phys. Chem. 92, 717 (1988).

548. W. Fink and E. W. Lang, J. Phys. Chem. 92, 6440 (1988).

549. W. Fink, H. RadKowitsch and E. W. Lang, Chem. Phys. 124, 239 (1988).

550. W. FinK, H. RADKOWITSCH and E. W. LANG, $Z$. Naturforsch. 43a, 538 (1988).

S5I. E. W. LANG, W. FINK, H. RadkowitsCh and D. GiRlich, Ber. Bunsenges. Phys. Chem. 94,342 (1990).

552. E. W. LANG, W. FINK and H. RADKowItsCh, Hydrogen-Bonded Liquids, NATO ASI Ser.C. Vol. 329, Eds. J. DORE and J. Teixeira, p. 393, Kluwer, The Netherlands (1991).

553. H. Kanno and C. A. Angell, J. Phys. Chem. 81, 2639 (1977),

554. C. A. Angell and E. J. Sare, J. Chem. Phys. 52, 1058 (1970).

555. H. Kanno, I. Shirotani and S. K. Minomura, Bull. Chem. Soc. Jpn. 53, 2079 (1980).

556. C. A. Angell, E. J. Sare, J. Donmella and D. R. Mac Farlane, J. Phys. Chem. 85, 1461 (1981).

557. H. Kanno, I. Shirotani and S. Minomura, Bull. Chem. Soc. Jpn. 54, 2607 (1981).

558. D. R. Mac Farlane, R. K. Kadyala and C. A. Angel., J. Phys. Chem. 87, 235 (1983).

559. H. Kanno, J. Phys. Chem. 91, 1967 (1987).

560. C. A. Angell and J. C. TuCKer, J. Phys. Chem. 84, 268 (1980).

561. I. C. Baianu, N. Boden, D. Lightowlers and M. Mortimer, Chem. Phys. Lett. 54, 169 (1978).

562. N. BODEN and M. MORTIMER, J. Chem. Soc. Faraday Trans. II 74, 353 (1978).

563. E. J. Sutter and J. F. Harmon, J. Phys. Chem. 79, 1958 (1975).

564. J. F. Harmon and E. J. SuTter, J. Phys. Chem. 82, 1938 (1978).

565. R. G. Palmer, Heidelberg Colloquium on Glassy Dynamics. Lecture Notes in Physics, Vol. 275, Eds. J. L. VaN HEMmEN and I. Morgenstern, Springer, Berlin (1987).

566. P. TABorek, R. N. Kleiman and D. J. Bishop, Phys. Rev. B 34, 1835 (1986).

567. H. G. Hertz, G. Keller and H. Versmold, Ber. Bunsenges. Phys. Chem. 73, 549 (1969).

568. G. Keller, Ber. Bunsenges. Phys. Chem. 76, 24 (1972).

569. H. G. HerTZ and C. RÄDLE, Ber. Bunsenges. Phys. Chem. 78, 509 (1974).

570. K. J. Müller and H. G. Hertz, Z. Phys. Chem. N.F. 140, 31 (1984).

571. R. Ahlrichs, H.J. Böhm, H. G. HerTZ and K. J. Müller, Z. Phys. Chem. N.F. 142, 67 (1984).

572. H. Radkowitsch and E. W. Lang, Ber. Bunsenges. Phys. Chem. 96, 162 (1992).

573. J. L. FinNey and J. TURNeR, Discuss. Faraday Soc. 85, 1 (1988).

574. P.-O. Eriksson, G. Lindrlom, E. E. Burnell and G. Tiddy, J. Chem. Soc. Faraday Trans. I 84, 3129 (1988).

575. W.-Y. WEN, Water and Aqueos Solutions, Ed. R. A. HORNE, Wiley (1972).

576. J. Turner, A. K. SOPer and J. L. Finney, Mol. Phys. 70, 679 (1990).

577. E. W. Lang, S. Bradi, W. Fink, H. Radkowitsch and D. Girlich, J. Phys. Condens. Matter 2, SA 195 (1990).

578. H. Kanno, K. Shimada and T. Katoh, J. Phys. Chem. 93, 4981 (1989).

579. L. H. I. U. Mettananda and R. J. Speedy, J. Phys. Chem. 88, 4163 (1984).

580. S. BRADL and E. W. LANG, in preparation. 